\title{
ASSESSMENT OF THE ENVIRONMENTAL IMPACTS OF COALBED METHANE DEVELOPMENT IN THE POWDER RIVER BASIN - Use of Coalbed Methane Produced Water for Cropland Irrigation
}

\section{TOPICAL REPORT}

START DATE: January 2002

END DATE: December 2005

By

Jeff Morris

May 2008

Work Performed Under Cooperative Agreement

Task 30 under DE-FC26-98FT40323

For

Wolverine Corporation

And

U.S. Department of Energy

National Energy Technology Laboratory

Morgantown, West Virginia

By

Western Research Institute

Laramie, Wyoming

Kamalendu Das

Task 30 


\section{TABLE OF CONTENTS}

LIST OF TABLES AND FIGURES............................................................................. iii

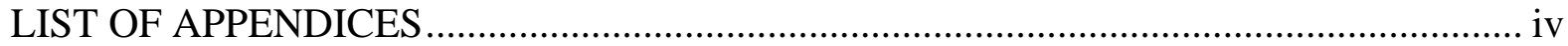

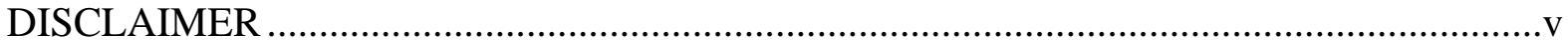

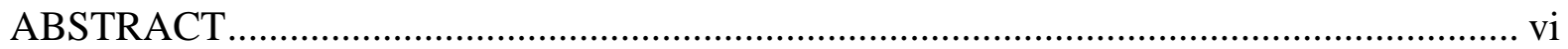

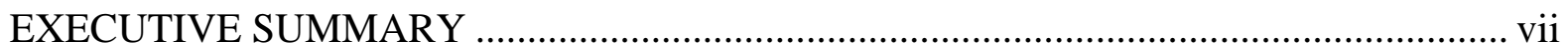

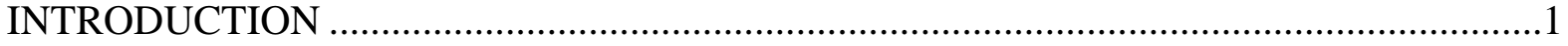

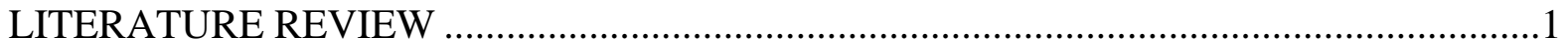

The Chemistry of Saline/Sodic Soils ...................................................................2

Impact of Sodicity/Salinity on Infiltration Rate and Hydraulic Conductivity ................5

The Effect of Exchangeable Magnesium on the Physical Properties of Soils ................5

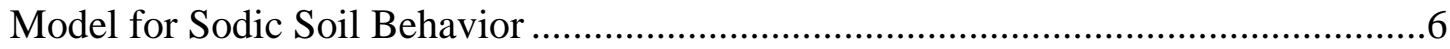

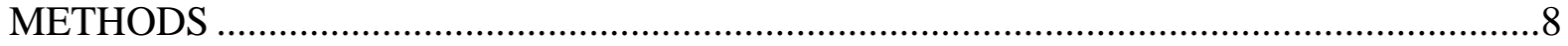

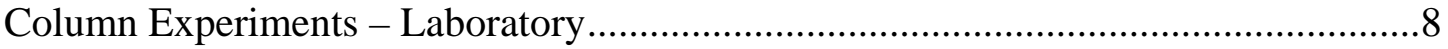

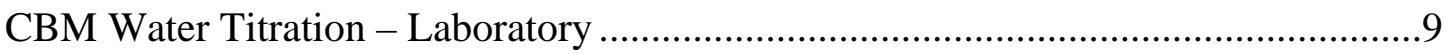

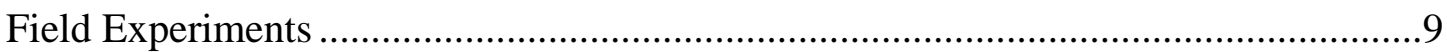

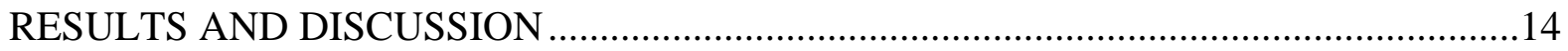

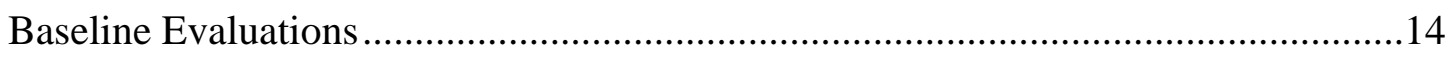

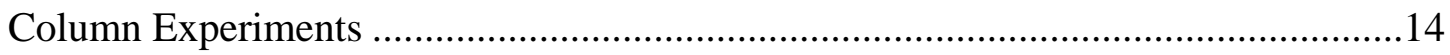

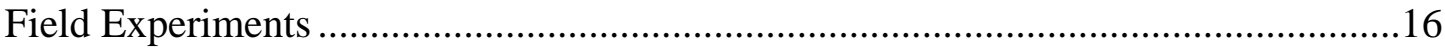

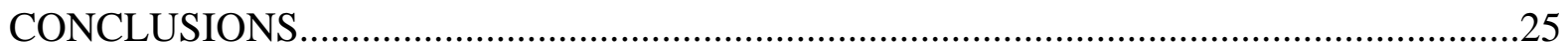

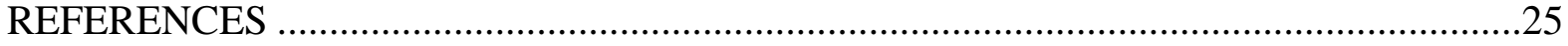




\section{LIST OF TABLES}

Table

$\underline{\text { Page }}$

1. Soil and Water Treatment Amendments to Study Plots at Cooksley Site. 11

2. Baseline Water Quality for Surface and Produced Water at Cooksley Site .15

\section{LIST OF FIGURES}

Figure

$\underline{\text { Page }}$

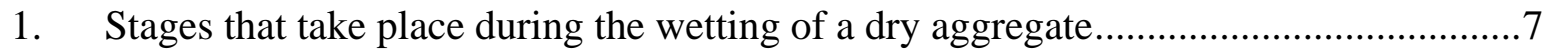

2. Laboratory setup for column studies ..................................................................

3. Sidewall of soil excavation pit ....................................................................... 10

4. Soil treatment (gypsum) being applied to field test site .........................................11

5. Location of soil amendments at the Cooksley Ranch site .......................................12

6. Locations of the CBM water treatments at the Cooksley site..................................12

7. Soil study pit and riser locations at Cooksley site ................................................13

8. Hydraulic conductivity of treated and untreated soil cores from Leiter and Cooksley sites .15

9. Hydraulic conductivity of soil cores from Leiter site irrigated with surface and CBM water. 16

10. Sodium concentrations in soil horizon A following irrigation at Cooksley site .18

11. Sodium concentrations in soil horizon Bt-1 following irrigation at Cooksley site......19

12. Sodium concentrations in soil horizon Bt-2 following irrigation at Cooksley site......20

13. SAR values in soil horizon A following irrigation at Cooksley site.........................21

14. SAR values in soil horizon Bt-1 following irrigation at Cooksley site ......................22

15. SAR values in soil horizon Bt-2 following irrigation at Cooksley site ......................23

16. Comparison of soil chemistry after certain treatments at Cooksley site.....................24 


\section{LIST OF APPENDICES}

1. Appendix I - Soil Survey of Cooksley and Leiter Sites .......................................... 27

Appendix IA - Leiter Field \#1 Soil Profile Descriptions ........................................ 38

Appendix IB - Cooksley Ranch Study Site Soil Profile Descriptions ....................... 49

2. Appendix II - Baseline Chemistry of Surface and CBM Produced Water....................... 59

3. Appendix III - Chemical Analysis of Soil Cores Used in Laboratory Experiments .......... 61

4. Appendix IV - Baseline Soil Infiltration of Cooksley Site .......................................... 66

5. Appendix V - Baseline Soil Chemistry at Cooksley Site: Fall 2003 ............................ 90

6. Appendix VI - Baseline Soil Chemistry at Cooksley Site: Spring 2004......................... 100

7. Appendix VII - Baseline Soil Chemistry at Cooksley Site: Fall 2004............................ 110 


\section{DISCLAIMER}

This report was prepared as an account of work sponsored by an agency of the United States Government. Neither the United States Government nor any agencies thereof, nor any of its employees makes any warranty, expressed or implied, or assumes any legal liability or responsibility for the accuracy, completeness, or usefulness of any information, apparatus, product, or process disclosed or represents that its use would not infringe on privately owned rights. Reference herein to any specific commercial product, process, or service by trade name, trademark, manufacturer, or otherwise does not necessarily constitute or imply endorsement, recommendation, or favoring by the United States Government or any agency thereof. The views and opinions of authors expressed herein do not necessarily state or reflect those of the United States Government or any agency thereof. 


\begin{abstract}
Water quality is a major concern with regard to development of coalbed methane (CBM) in the Powder River Basin, Wyoming. Large quantities of water are being produced and discharged as a by-product in the process of releasing natural gas from coal. Current practices of discharging large volumes of water into drainage channels or using it to irrigate cropland areas has the potential to elevate salinity and sodicity in soils. Elevated salinity affects the ability of plants to uptake water to facilitate biochemical processes such as photosynthesis and plant growth. Elevated sodicity in irrigation water adversely affects soil structure necessary for water infiltration, nutrient supply, and aeration. Salinity and sodicity concentrations are important in that a sodic soil can maintain its structure if the salinity level is maintained above the threshold electrolyte concentration. In this study, cropland soil and CBM water were treated with gypsum and sulfur. Changes in soil chemistry among different treatments were monitored using a split plot experiment. The CBM water used for irrigation had an EC of $1380 \mu \mathrm{S} \mathrm{cm}^{-1}$ and SAR of 24.3 $\mathrm{mmol}^{1 / 2} \mathrm{~L}^{-1 / 2}$. Baseline and post treatment soil samples were collected to a depth of $60 \mathrm{~cm}$ within each study plot, analyzed, and characterized for chemical parameters.

Comparisons between Spring 2004 and Fall 2004 soil chemistry data after one irrigation season (using the equivalent of 1 month of irrigation water or 12 inches) indicated that irrigating with Piney Creek water or a 50:50 blend of Piney Creek water and CBM water did not cause SAR values to increase. A combination of using a gypsum amendment to the soil along with a gypsum injection and sulfur burner treatment to the irrigation water resulted in the lowest SAR value in the first soil horizon among treatments irrigated solely with CBM produced water. The SAR value resulting from this combination treatment was 53\% lower than using CBM water with no amendments to soil or water. Soil amendments to cores in the laboratory did not increase soil hydraulic conductivity when CBM water was applied.
\end{abstract}




\section{EXECUTIVE SUMMARY}

Water quality is a major concern with regard to development of coalbed methane (CBM) in the Powder River Basin, Wyoming. Large quantities of water are being produced and discharged as a by-product in the process of releasing natural gas from coal. Current practices of discharging large volumes of water into drainage channels or using it to irrigate cropland areas has the potential to elevate salinity and sodicity in soils. Elevated salinity affects the ability of plants to uptake water to facilitate biochemical processes such as photosynthesis and plant growth. Elevated sodicity in irrigation water adversely affects soil structure necessary for water infiltration, nutrient supply, and aeration. Salinity and sodicity concentrations are important in that a sodic soil can maintain its structure if the salinity level is maintained above the threshold electrolyte concentration. In this study, cropland soil and CBM water were treated with gypsum and sulfur. Changes in soil chemistry among different treatments were monitored using a split plot experiment. The CBM water used for irrigation had an EC of $1380 \mu \mathrm{S} \mathrm{cm}^{-1}$ and SAR of 24.3 $\mathrm{mmol}^{1 / 2} \mathrm{~L}^{-1 / 2}$. Baseline and post treatment soil samples were collected to a depth of $60 \mathrm{~cm}$ within each study plot, analyzed, and characterized for chemical parameters.

Comparisons between Spring 2004 and Fall 2004 soil chemistry data after one irrigation season (using the equivalent of 1 month of irrigation water or $\sim 12$ inches) indicated that irrigating with Piney Creek water or a 50:50 blend of Piney Creek water and CBM water did not cause SAR values to increase. A combination of using a gypsum amendment to the soil along with a gypsum injection and sulfur burner treatment to the irrigation water resulted in the lowest SAR value in the first soil horizon among treatments irrigated solely with CBM produced water. The SAR value resulting from this combination treatment was 53\% lower than using CBM water with no amendments to soil or water. Soil amendments to cores in the laboratory did not increase soil hydraulic conductivity when CBM water was applied. 


\section{INTRODUCTION}

Water quality issues are at the forefront with regard to the development of coalbed methane (CBM) reserves in the Powder River Basin. The practice of discharging large volumes of water into drainage channels or using it to irrigate rangeland areas has the potential of causing serious problems with regard to salinity and sodicity of soils. The primary problems associated with salinity are related to the ability of plants to take up water to facilitate the biochemical processes of photosynthesis and plant growth. As the solution electrolyte concentrations of the soil increase, plants (dependent on their level of salt tolerance) will become less able to absorb sufficient amounts of water. As a result, the plants will grow at slow rates or die.

It is very important to make sure that water applied to soils meets the favorable combination of salinity and sodicity that will allow the plants to grow at good production levels and that will maintain the structure of the soils. Each soil will react differently to the chemistry of the water applied and the method of application. Therefore, a research effort that includes studies of the interactions of various parameters with the soil types found in the area should be conducted so that produced water from CBM operations can be used in an environmentally safe manner.

\section{LITERATURE REVIEW}

Large quantities of water are being produced and discharged as part of the process of releasing CBM from coal seams. The excess of produced water is causing concerns from several points of view. The drawdown of water in the coal aquifers is of major concern for landowners and others such as those worried about future generations and the availability of water. In addition, the dilemma of how to use the water in worthwhile, environmentally sound applications is currently under consideration. The practice of discharging large volumes of water into drainage channels or using it to irrigate rangeland areas has the potential to cause serious problems with regard to salinity and sodicity of soils. Some waters currently being disposed of in the Power River Basin are of such quality that land application should not cause any significant problems. However, there is concern that some of the CBM produced waters may negatively impact irrigated croplands (RIENR 2005).

The primary problems associated with salinity are related to the ability of plants to uptake water to facilitate the biochemical processes of photosynthesis and plant growth. As the solution electrolyte concentrations of the soil increase, plants (dependent on their level of salt tolerance) will become less able to absorb sufficient amounts of water. As a result, the plants will grow at slow rates or die.

The major impact of sodicity on the soils is associated with soil structure. Soil structure is important to maintain the flow of gases (oxygen) and solution (water plus nutrients) to the plant roots and can cause severe erosion of once-productive soils. Dependent on the characteristics of individual soils, as the level of sodium in the soil increases the physical 
structure will degrade. High levels of sodium can cause the structure of a soil to completely disperse.

An important aspect of the sodicity and salinity chemistry of soils is that the two are closely related. For example, a highly sodic (high sodium adsorption ratio (SAR)) soil can maintain its soil structure if the salinity level (electrical conductivity (EC)) of the soil is high. However, if the salinity level is low (the water is very clean), the highly sodic soil will slake and disperse and the structure will be lost. If a soil is characterized by a low SAR, the application of clean water or water characterized by low EC can cause degradation of soil structure. If the electrolyte concentration of the water applied to the soil is high, the soils will maintain their structure.

The dilemma of this system is to make sure that water applied to soils meets the favorable combination of salinity and sodicity that will allow the plants to grow at good production levels and that will maintain the structure of the soils. Each soil will react differently to the chemistry of the water applied and the method of application. Therefore, research that includes studies of the interactions of these various parameters with the soil types found in the area should be conducted so that produced water from CBM operations can be discharged in an environmentally safe manner.

Several issues must be understood in order to successfully manage CBM-produced water for land application, including the chemistry of saline/sodic soils and its impact on the infiltration rate and hydraulic conductivity, as well as the effect of exchangeable magnesium on the physical properties of soils.

\section{The Chemistry of Saline/Sodic Soils}

Excessive levels of salts impact large areas of soils around the world. The primary impacts of salts on soil quality are associated with saline and sodic conditions. Saline soil conditions are associated with excess salts usually consisting of chlorides $\left(\mathrm{Cl}^{-1}\right)$ and sulfates $\left(\mathrm{SO}_{4}{ }^{-2}\right)$ of sodium $\left(\mathrm{Na}^{+1}\right)$, calcium $\left(\mathrm{Ca}^{+2}\right)$, and magnesium $\left(\mathrm{Mg}^{+2}\right)$ (Sumner et al., 1998). Saline conditions often have a deleterious effect on plant growth because salts decrease the osmotic potential of soil water, making it difficult for plants to extract water. Sodic conditions are associated with elevated levels of $\mathrm{Na}$ on the exchange complex, often responsible for the development of the poor physical conditions in a soil. Thus, the impacts of Na result in an inadequate balance between water and air regimes in the soil. This imbalance is caused by restricted water infiltration and transmission properties causing the soil to be too wet or dry for much of the time, resulting in poor root development and plant growth. In addition, sodic soils often are difficult to cultivate and have low load bearing properties. Poor structural stability promotes the sealing of soil pores and crust formation at the soil surface, resulting in soil erosion and pollution of surface water resources. 
The definition of a sodic soil in simple terms is a soil that has been adversely impacted physically by the presence of $\mathrm{Na}$ adsorbed to the cation exchange sites. The presence of $\mathrm{Na}$ in a soil promotes the slaking of aggregates and the dispersion of clay particles. At the same time, the impact of $\mathrm{Na}$, as characterized by SAR or ESP, on the physical character of a soil is greatly dependent on the salinity of the soil. It is impossible to estimate the impact of low or high SAR values on the physical state of a soil or spoil material without evaluating the EC of the system (Shanmuganathan and Oades, 1983). Any attempt to set critical ESP or SAR values for land management would be arbitrary unless total cation concentration or EC is taken into consideration simultaneously (Sumner et. al., 1998). Research has shown that extremely high SAR values do not cause physical degradation of soil materials if the system also contains high levels of salts. This fact was first demonstrated by research done by Quirk and Schofield (1955). Their work demonstrated that soil materials with an ESP of 40 maintained a stable permeability with an electrolyte concentration of about $30 \mathrm{mmol} / \mathrm{L}$ (about EC $=2.1 \mathrm{dS} / \mathrm{m}$ ). McNeal and Coleman (1966) pointed out that typical arid land soils (having clay mineralogy dominated by 2:1 layer silicates with only moderate amount of montmorillonite) can tolerate ESP values of 15 or greater before serious reductions in hydraulic conductivity occur, if the salt concentration of the percolating solution exceeds $3 \mathrm{mmol} / \mathrm{L}(0.2 \mathrm{dS} / \mathrm{m})$. Gardner et al. (1959) came to the same conclusion dealing with unsaturated soils. Sumner et al. (1998) discussed the SAR/EC relationship very thoroughly in their publication titled "Sodic Soils: Distribution, Properties, Management, and Environmental Consequences." The discussion addresses the fact that the USDA Soil Salinity Laboratory did not address this topic in their recommendations set forth during the late 1950s for the management of sodic soils, even though they were well aware of it. In fact, the definition of sodic soils was published by USSL (1954) without mention of electrolyte concentration in the definition. It was apparent at the time that people were well aware of the impact of salinity on the behavior of sodic conditions; however, apparently these scientists elected to downplay the relationships because of the apparent transient nature of salinity. Sumner et al. (1998) noted that if the work of Quirk and Schofield (1955) had been adopted in the United States, a much clearer understanding of sodicity would have resulted. Instead of using electrolyte concentration as an important component of the definition of sodic soils, Handbook 60, which was published in 1954 and reprinted in 1969 without emphasizing the importance of salinity and its impact on sodicity, has been and continues to be used as an authority addressing salinity and sodicity issues.

The impact of sodicity on the physical properties of soils is dependent on the electrolyte concentration associated with the system. If salt is added to a dispersed clay in a suspension, the increased electrolyte concentration causes the clay particles to stick together, forming flocs that settle. The minimum electrolyte concentration required to cause flocculation is referred to as the threshold electrolyte concentration (TEC) or flocculation value (FV). This value is dependent on counter-ion valency and clay type. The TEC values for a sodium-montmorillonite were shown to be about $12 \mathrm{~mol} / \mathrm{m}^{3} \mathrm{NaCl}$ or $0.86 \mathrm{dS} / \mathrm{m}$ and $0.25 \mathrm{~mol} / \mathrm{m}^{3} \mathrm{CaCl}_{2}$ or $0.02 \mathrm{dS} / \mathrm{m}$ for calcium montmorillonite (Van Olphen, 1977). Corresponding values for sodium and calcium illites were found to be $40 \mathrm{~mol} / \mathrm{m}^{3}$ to $50 \mathrm{~mol} / \mathrm{m}^{3} \mathrm{NaCl}$ and $0.25 \mathrm{~mol} / \mathrm{m}^{3}$, respectively (Arora and Coleman, 
1979). These data show that a sodium montmorillonite can be maintained in a flocculated condition if the salt levels of the same ion ( $\mathrm{Na}$ ) are about $1 \mathrm{dS} / \mathrm{m}$. Sodium illites will tend to remain flocculated if salt levels with the same ion (Na) are about $3.6 \mathrm{dS} / \mathrm{m}$. Sposito (1989) indicated through his discussion of the literature that a fully Na-saturated smectite suspension will flocculate if the electrolyte concentration is $>8 \mathrm{~mol} / \mathrm{m}^{3}(0.6 \mathrm{dS} / \mathrm{m})$ and a suspension of $\mathrm{Na}$ illite will do the same if the electrolyte concentration reaches about $50 \mathrm{~mol} / \mathrm{m}^{3}(3.6 \mathrm{dS} / \mathrm{m})$. His conclusion is that soil salinity tends to counteract the effect of exchangeable sodium on soil structure. The presence of divalent ions such as Ca would lower the TEC to lower salt concentrations.

The data show that if salinity is maintained at or above the TEC value for a specific material, the physical condition of the material will be maintained in a flocculated state, no matter how high the SAR. The only caveat to this situation is that some materials that have high SAR and EC character can become dispersed at the surface if impacted with water containing low levels of electrolytes from irrigation or rainfall. In addition, mechanical forces resulting from raindrop impact, the flow of water at the surface, or the use of farm equipment could cause clay dispersion. If measures are taken to eliminate these potential impacts to the system, the high-SAR soil/spoil material will usually be maintained in good physical condition. One method of doing this is to treat the surface with an amendment such as gypsum. Another method of protecting the surface against the mechanical forces that can initiate slaking and dispersion is to cover such spoils with topdressing material. This fact was demonstrated during a recent visit to the La Plata Mine. A soil pit was dug at a reclaimed site that had about 10 inches of topdressing material over spoil material that exceeded the current SAR standards. The spoil material had SAR values from 11 to 75 in the top four feet with EC values ranging from 3.5 to $7.3 \mathrm{dS} / \mathrm{m}$ at various depths within the same depth zone. The roots exhibited no problems growing through this material. In fact, the rooting showed numerous branching and overall good health. The spoil materials to about four inches below the topdressing/spoil interface showed evidence of weathering with the development of a very porous structure. Migration of $\mathrm{Na}$ at or near the topdressing/spoil interface was not evident, probably because the water present in the profile was readily removed via transpiration. The elevated SAR values associated with the spoil did not have a detrimental impact on plant growth.

It is generally accepted that both high SAR and/or low EC values create soil structural problems; however, we still do not have reliable criteria and standards for predicting how these parameters quantitatively affect structural stability and hydraulic conductivity (K) of soils (Rhoades, 1972). The mechanisms that cause these problems have been postulated to be: 1) swelling of soils, 2) clay dispersion and subsequent plugging of conducting pores by dispersed clay, and 3) failure of soil aggregates. However, the literature demonstrates divergence of opinions relative to the importance of these mechanisms. McNeal and Coleman (1966) and Jayawardane (1979) determined that clay swelling was the dominant mechanism reducing $\mathrm{K}$ in sodic soils. Research by Rhoades and Ingvalson (1969), Frenkel et al. (1978), Pupisky and Shainberg (1979), Shainberg et al. (1992), and Yousaf et al. (1987) showed that clay dispersion 
was the dominant mechanism responsible for the reduction in $\mathrm{K}$. Other scientists such as Waldron and Constantin (1970) and Cass and Sumner (1982) concluded that the reduction in K was primarily related to aggregate slaking caused by internal swelling pressure or from shearing stresses.

As noted previously, the chemistry associated with sodic and saline conditions in soils is very complex. The complexities of sodicity and salinity with regard to the management of soil materials will be discussed in some detail in the following review of the literature. The objective of this work is to show that an understanding of the chemistry associated with sodic/saline conditions relative to water/soil interactions can lead to the successful use of CBM-produced waters for improving vegetation production in various vegetation communities.

\section{Impact of Sodicity/Salinity on Infiltration Rate and Hydraulic Conductivity}

Hydraulic conductivity $(\mathrm{K})$ is less sensitive to sodic conditions as compared to the infiltration rate (IR). The primary factor responsible is the influence of mechanical energy resulting from the impact of raindrops at the surface. This mechanical energy promotes the disintegration of aggregates and the dispersion of clays, resulting in the formation of a structural crust. Crusts are formed as a result of the physical disintegration of aggregates, while compaction is caused by the impact of the water droplets and chemical dispersion of clays near the surface. Smectitic soils are very sensitive to reduced IR, even at very low ESP levels $(\mathrm{ESP}<3)$. A very important factor in the degradation of IR is that rainfall contains very low levels of salts. Therefore, rainfall is responsible for leaching electrolytes from the surface, leaving the surface materials more susceptible to dispersion. Hydraulic conductivity $(\mathrm{K})$ is much less susceptible to degradation, except at the surface, mainly because of the reduced impact of mechanical forces and the electrolyte levels that often occur (Shainberg et al., 1992). The mineral phase associated with arid land materials usually readily weathers, providing electrolytes to the system and enhancing its ability to maintain structure.

This discussion supports the fact that topdressing placed over sodic materials will alleviate the development of poor physical conditions in the sodic materials. The surface layer of material (topdressing) eliminates the mechanical energy input from raindrop impact and/or surface water flow and the low electrolyte water that would promote aggregate slaking and clay dispersion. Weathering of the surface layer and the underlying sodic materials would result in increased electrolyte levels in solution that would prevent dispersion; and the $\mathrm{Ca}^{+2}$ present in the solution would tend to self-remediate the sodic condition.

\section{The Effect of Exchangeable Magnesium on the Physical Properties of Soils}

The USSL (1954) grouped Ca and Mg together as similar ions beneficial in developing and maintaining soil structure. However, evidence now exists that shows that $\mathrm{Mg}$ can cause the deterioration of soil structure under certain conditions. Studies have shown that Na-Mg soils 
developed lower $\mathrm{K}$ characteristics than Na-Ca soils under similar conditions. Research has demonstrated that $\mathrm{Mg}$ can impact $\mathrm{K}$ through direct effects (specific effects) and through the ability of $\mathrm{Mg}^{+2}$ to cause higher exchangeable $\mathrm{Na}^{+1}$ levels to develop in the soils as compared to $\mathrm{Ca}^{+2}$ (Chi et al., 1977; Emerson and Chi, 1977).

Alperocitch et al. (1981) showed that well-weathered soils that do not contain $\mathrm{CaCO}_{3}$ are impacted by the specific effect of $\mathrm{Mg}^{+2}$. Reduction in $\mathrm{K}$ and enhanced clay dispersion resulted when Na-Mg soils were leached with distilled water (simulated rain water). The theory behind this finding was that clays saturated with $\mathrm{Mg}^{+2}$ are chemically more stable and do not release electrolytes into solution. As a result, the lack of weathering products will allow the system to disperse more easily when leached with water that contains low concentrations of electrolytes. In the same study, exchangeable $\mathrm{Mg}^{+2}$ was found not to have a specific effect on the $\mathrm{K}$ and clay dispersion in calcareous soils. In these soils, $\mathrm{Mg}^{+2}$ enhances the dissolution of $\mathrm{CaCO}_{3}$, increasing the solution electrolyte concentrations and preventing the dispersion of clays and the reduction of $\mathrm{K}$.

\section{Model for Sodic Soil Behavior}

In the past, soil scientists have used a model involving the electrical diffuse double-layer theory to explain sodic soil behavior. This explanation was usually conducted using pure clay minerals in saturated systems. However, in natural systems, complex clay systems are bound together into aggregates with silt and sand particles by inorganic and organic compounds and are usually not suspended in water. The slaking of aggregates and the dispersion of clays requires forces other than those that operate in colloidal clay suspensions. Rengasamy and Sumner (1998) have developed a model that describes the processes that take place during the wetting of a dry soil aggregate. Their model will be presented herein as a realistic approach describing the influences of sodicity and salinity on the physical nature of natural soil systems.

The model consists of four stages. Dry soil aggregates are held together by inorganic and organic compounds and associated bonds that produce very strong attractive forces. The forces involved include Lifshitz-van der Waals, ionic bonds, hydrogen, hydrophobic and hard-soft acidbase reactions. As dry aggregates are wetted, solvation or hydration forces become important. The stability of aggregates, and hence the pore systems, depends on attractive and repulsive forces resulting from intermolecular and electrostatic interactions between the soil solution and soil particles (Rengasamy and Olsson, 1991). When an aggregate is placed in contact with water, the interactive forces lower the potential energy of water molecules, thus releasing energy for structural changes and as heat. Aggregate slaking, clay swelling, and clay dispersion are the major mechanisms that occur during these transformations.

Thus, as an aggregate is hydrated, the initial attractive forces between clay particles decrease. As hydration increases, the distance between particles increases. In general, if the clay particles are saturated with $\mathrm{Ca}$ or $\mathrm{Mg}$, additional hydration does not increase the interparticle 
distance beyond a few nanometers, resulting in a net force that is attractive and the aggregates are held together by hydrated cations. The swelling resulting from these reactions will occur even with high electrolyte concentrations. Slade and Quirk (1991) found that the change in separation to $1.5 \mathrm{~nm}$ is not affected by electrolyte solution (crystalline swelling) and that the separation from 1.5 to $1.9 \mathrm{~nm}$ is an osmotic process that includes electrolyte concentrations, charge density, and the location of the charge in the clay minerals. If the clays are saturated by monovalent cations such as $\mathrm{Na}$, the clay particles are separated beyond $7 \mathrm{~nm}$ dependent on the ionic strength and the existence of soft-hard acid-base reactions. This results in clay dispersion, shown as Stage 3 (Figure 1). Stage 3 can also be reached when a source of mechanical energy is applied to the clay domains that have undergone limited separation. Mechanical energy resulting from raindrop impact and surface water flow can overcome the attractive forces, causing the system to separate. Once the system is completely dispersed the electrostatic repulsive forces, as predicted by the electrical double-layer theory, become important to the physical nature of the system. A dispersed clay system will become flocculated as the difference in the electrical potentials in the inner and outer solutions approach zero and as the clay particles approach each other. The repulsive pressure is balanced by osmotic pressure, and the van der Waals attractive forces become dominate. At this point, the clays become flocculated, as identified as Stage 4 of the model.

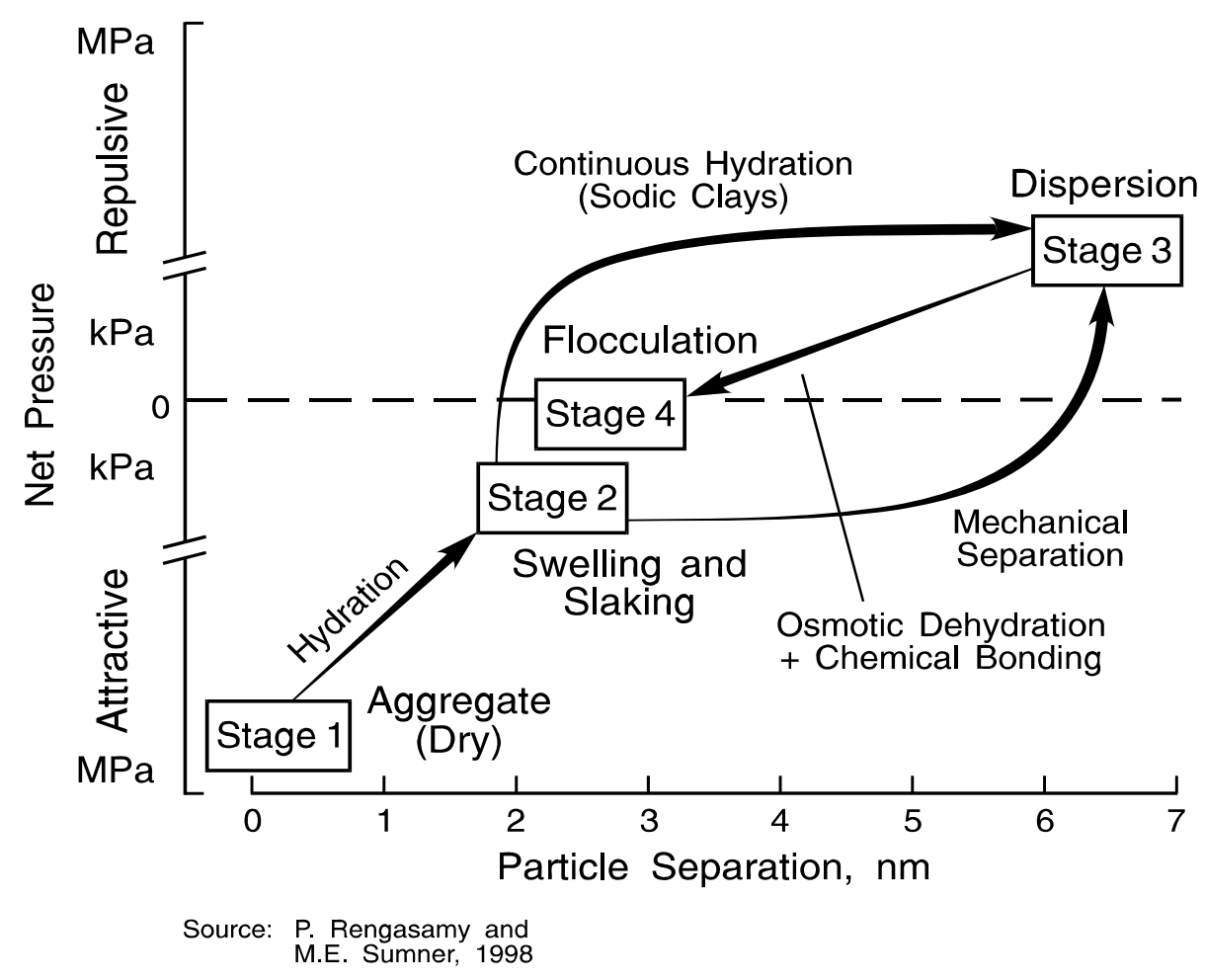

Figure 1. Stages that take place during the wetting of a dry aggregate soil.

Since much of the research regarding sodic/saline conditions has been conducted using saturated aqueous systems, some concern exists for whether these studies apply to unsaturated 
conditions. Since low water content results in low repulsion forces, unsaturated systems would be expected to have higher attractive forces as compared to saturated systems. Rengasamy and Sumner (1998) indicated that spontaneous dispersion takes place when sodic clay is impacted with water of very low electrolyte concentration. However, water content below saturation results in limited swelling and incomplete separation of clay particles with the interparticle distance depending upon the water content. Therefore, aggregate slaking and clay dispersion in unsaturated systems would be limited as compared to saturated systems for specific SAR/EC conditions. Russo and Bresler (1977) demonstrated this fact in their study evaluating hydraulic conductivity with regard to cationic ratio $\left(\mathrm{Na}^{+1} /\left(\mathrm{Ca}^{+2}\right)^{1 / 2}(\mathrm{R})\right.$, electrolyte concentrations (C), and volumetric water content $(\theta)$. This study showed that under specific combinations of these variables the value of the cationic ratio can be estimated for a given hydraulic conductivity. For example, under saturated conditions with $\mathrm{C}=0.02 \mathrm{~N}$ (approximate irrigation field soil) it is possible to maintain $\mathrm{K} \geq 0.5 \mathrm{~cm} / \mathrm{s}$ as long as $\mathrm{R} \leq 14$. Under the same conditions ( $\mathrm{C}$ and $\mathrm{K}$ ), but in unsaturated soils the corresponding value for $\mathrm{R}$ is 20 . This study shows that water of poorer quality (higher sodicity) can be applied when unsaturated conditions are maintained during irrigation. These studies demonstrate that the negative effect of high SAR and low solution electrolyte levels decreases in dryer soils.

\section{METHODS}

\section{Column Experiments - Laboratory}

A laboratory study was conducted to determine saturated hydraulic conductivity of soil cores prepared using materials from potential project locations. The effects of product water on hydraulic conductivity were evaluated after addition of different treatments of gypsum and sulfuric acid.

Water samples were collected from CBM sources to be used for irrigation on the Cooksley Ranch Site in Northeast Wyoming. Water samples were collected in 5-gallon containers for laboratory testing. The container was purged with argon to reduce the incorporation of oxygen into the water during collection. Field parameters tested included temperature (T), pH, EC, and Eh. Samples collected for initial characterization were filtered $(0.45 \mu \mathrm{m})$ and stored at $4^{\circ} \mathrm{C}$ during shipment to the laboratory. Sample containers were preconditioned as required by Environmental Protection Agency methods for the various constituents.

Soil material for the laboratory studies was collected from two potential irrigation study sites, the Cooksley Ranch and the Leiter Ranch, located near Buffalo, Wyoming. The materials were collected and placed in 5-gallon buckets and shipped to Western Research Institute (WRI), Laramie, Wyoming. The materials were stored at $4^{\circ} \mathrm{C}$ at field moisture conditions until prepared for analyses. Sub-samples of homogenized soil materials were analyzed to establish baseline chemistry. Saturated paste extracts were prepared and analyzed for $\mathrm{pH}$, EC, saturation 
percentage, $\mathrm{Ca}, \mathrm{Mg}$, $\mathrm{Na}$, potassium $(\mathrm{K}), \mathrm{Cl}, \mathrm{SO}_{4}, \mathrm{SAR}$, texture, carbonate $\left(\mathrm{CO}_{3}\right)$, and bicarbonate $\left(\mathrm{HCO}_{3}\right)$.

CBM produced water was percolated through reconstructed soil columns for 4,500 minutes (75 hours; Figure 2). The resulting hydraulic conductivities associated with the various treatments were determined. The results of this work provided a basis of how the soil responds to increased levels of sodicity and salinity introduced during irrigation using CBM produced water and the effect of potential treatments.

\section{CBM Water Titration - Laboratory}

CBM co-produced water was titrated with $0.02 \mathrm{~N} \mathrm{HCl}$ to determine the $\mathrm{pH}$ at which the dominate carbonate species in solution shifted from bicarbonate $\left(\mathrm{HCO}_{3}{ }^{-}\right)$to carbonic acid $\left(\mathrm{H}_{2} \mathrm{CO}_{3}\right)$. The sulfur-burners at the field site were adjusted to achieve this $\mathrm{pH}$, therefore, shifting the carbonate equilibrium and releasing $\mathrm{CO}_{2}$ to the atmosphere. Decreasing the $\mathrm{HCO}_{3}{ }^{-}$ concentration in the water minimizes the possibility of $\mathrm{CaCO}_{3}$ precipitation in the field, which would decrease dissolved Ca concentrations and raise SAR values.

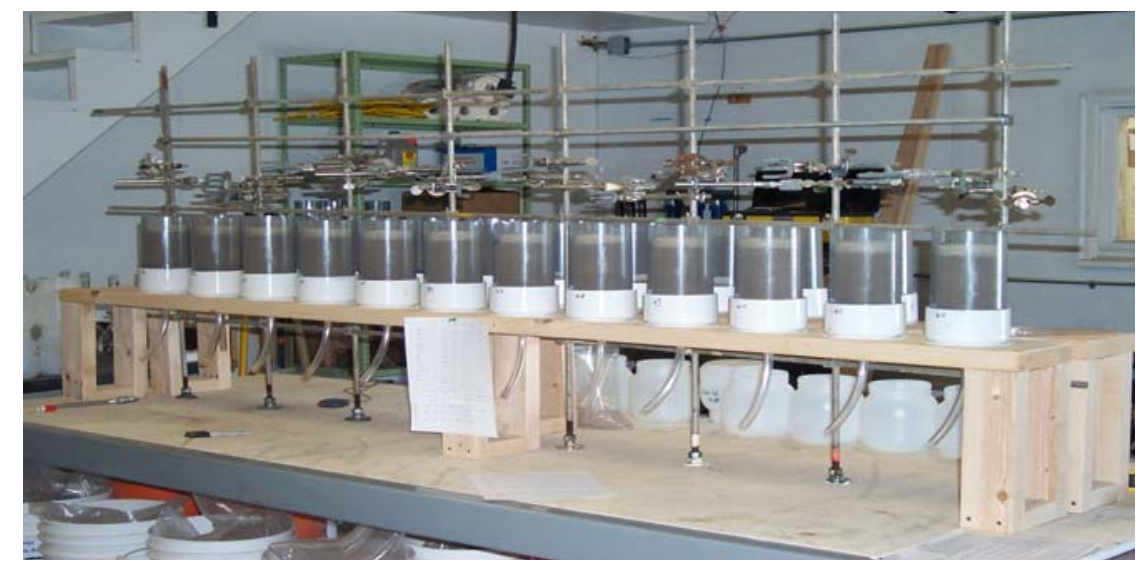

Figure 2. Laboratory set-up of soil column studies.

\section{Field Experiments}

Detailed Order II soil surveys were completed at two locations in the Powder River Basin to evaluate soil suitability for application of CBM product water. Test-pits were excavated (e.g., Figure 3) and soil profiles described to aid in the classification of predominant soil types and suitability for water application within each location. Soil samples were collected from representative profiles and analyzed for chemical and physical parameters. Analyses included $\mathrm{pH}$, electrical conductivity (EC), $\mathrm{Ca}, \mathrm{Mg}, \mathrm{Na}$, and textural class with percent sand, silt, and clay. In addition, tension infiltrometer and double ring permeameter tests were completed within each location area to estimate hydraulic conductivity. Bulk density was also determined by 
excavation. Based on analyses of these parameters, the Cooksley location was selected for CBM product water application.

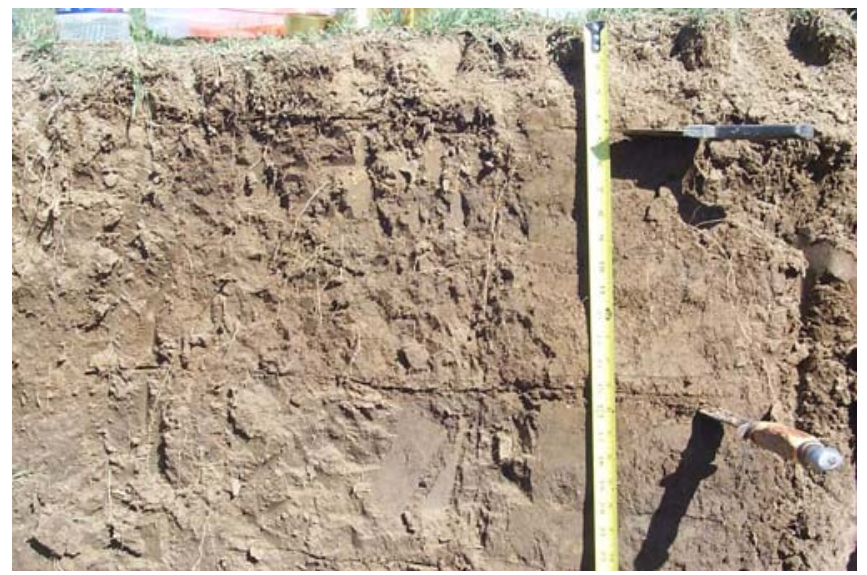

Figure 3. Sidewall of soil excavation test pit.

A split-plot in space statistical design was selected for the Cooksley project area. This design allows for analysis of interactions between soil and product water treatments on soil chemical and physical properties. Randomized study plots were established within the project area and baseline chemical and physical soil properties were measured. Chemical analysis included $\mathrm{pH}, \mathrm{EC}, \mathrm{Ca}, \mathrm{Mg}, \mathrm{Na}, \mathrm{SO}_{4}, \mathrm{Cl}$, and alkalinity. Unsaturated hydraulic conductivity was measured using tension infiltrometer measurements at -10 and $-6 \mathrm{~cm}$ tensions. Saturated hydraulic conductivity was estimated using Wooding's equation for unconfined infiltration rates into a soil from a circular source. Baseline data were analyzed statistically to determine variation between treatment plots prior to treatment application.

Soil amendments and water treatments were used to modify the soil/water conditions allowing irrigation with highly sodic CBM produced water. The treatments evaluated at the site included combinations of irrigation waters, soil amendments and water treatment. Soil treatments were applied to plots after baseline analyses were complete (e.g., Figure 4). Soil treatments included sulfur (0.5 t/ac), gypsum (1.5 t/ac), sulfur (0.5 t/ac) and gypsum (1.5 t/ac), and no treatment. These soil amendments were applied in Fall 2003 and Spring 2004. Product water treatments included the removal of bicarbonate using a sulfuric acid injection system, injection of solution grade gypsum, blending with typical irrigation water (50:50), no treatment, and typical irrigation water as the control (Table 1). A total of 12 inches of water was applied to the study area over the course of 1 irrigation season. A Gee passive capillary lysimeter was installed along with dielectric soil moisture probes to evaluate leaching fraction, drainage water quality, and irrigation efficiency. 


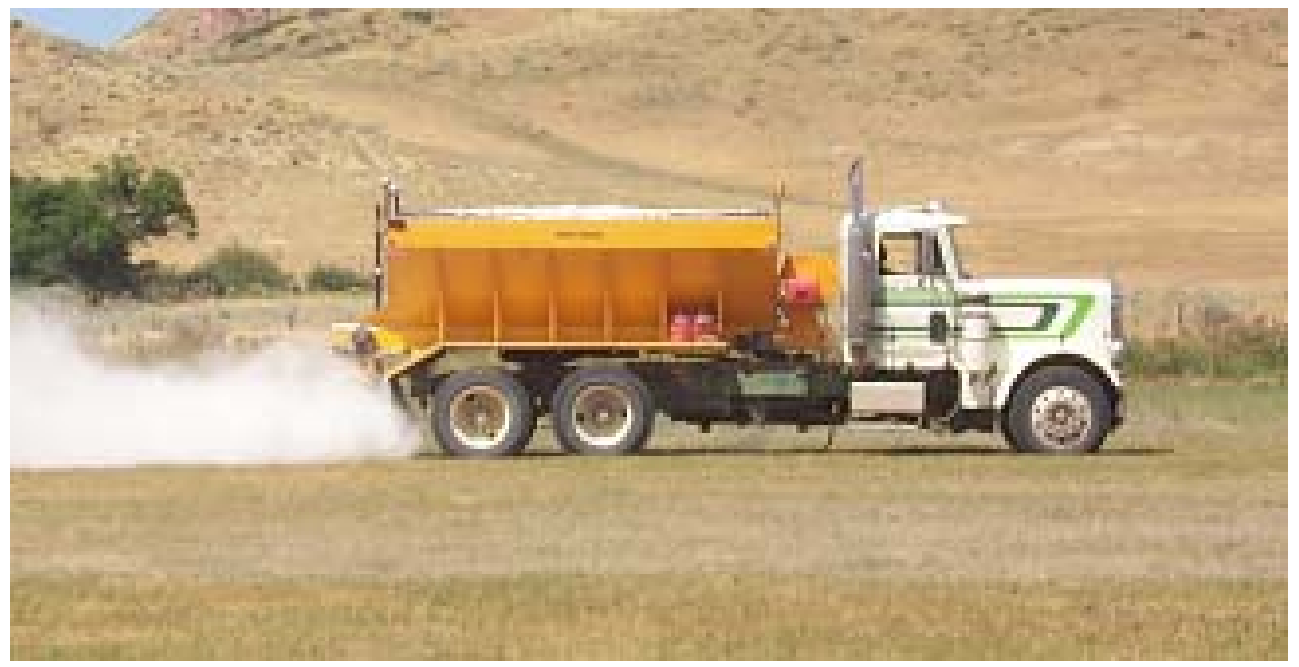

Figure 4. Soil treatment (gypsum) being applied to field test site.

Diagrams showing the location of soil amendment treatments and the CBM water treatments are provided in Figures 5 and 6, respectively. The soil amendment treatments were applied and irrigation was initiated during the fall of 2003 and continued during the 2004 growing season.

Table 1. Soil and water treatments applied to plots and irrigation water at the Cooksley Ranch site near Buffalo, Wyoming, during the 2004 irrigation season.

\begin{tabular}{|c|c|c|c|}
\hline Treatment & Water source & Water amendment & Soil amendment \\
\hline PC-NT & Piney Creek & none & none \\
\hline PC-G & Piney Creek & none & gypsum \\
\hline PC-S & Piney Creek & none & sulfur \\
\hline PC-GS & Piney Creek & none & gypsum + sulfur \\
\hline CBM-NT & CBM & none & none \\
\hline CBM-G & CBM & none & gypsum \\
\hline CBM-S & CBM & none & sulfur \\
\hline CBM-GS & CBM & none & gypsum + sulfur \\
\hline CBM-PC-NT & Blend $^{\mathrm{a}}$ & none & none \\
\hline CBM-PC-G & Blend & none & gypsum \\
\hline CBM-PC-S & Blend & none & sulfur \\
\hline CBM-PC-GS & Blend & none & gypsum + sulfur \\
\hline CBM-G-NT & CBM & gypsum injection & none \\
\hline CBM-G-G & CBM & gypsum injection & gypsum \\
\hline CBM-G-S & CBM & gypsum injection & sulfur \\
\hline CBM-G-GS & CBM & gypsum injection & gypsum + sulfur \\
\hline CBM-G-SB-NT & CBM & gypsum injection + sulfur burner & none \\
\hline CBM-G-SB-G & CBM & gypsum injection + sulfur burner & gypsum \\
\hline CBM-G-SB-S & CBM & gypsum injection + sulfur burner & sulfur \\
\hline CBM-G-SB-GS & CBM & gypsum injection + sulfur burner & gypsum + sulfur \\
\hline
\end{tabular}

${ }^{a}$ The blend used was a 50:50 ratio of creek water to CBM produced water. 

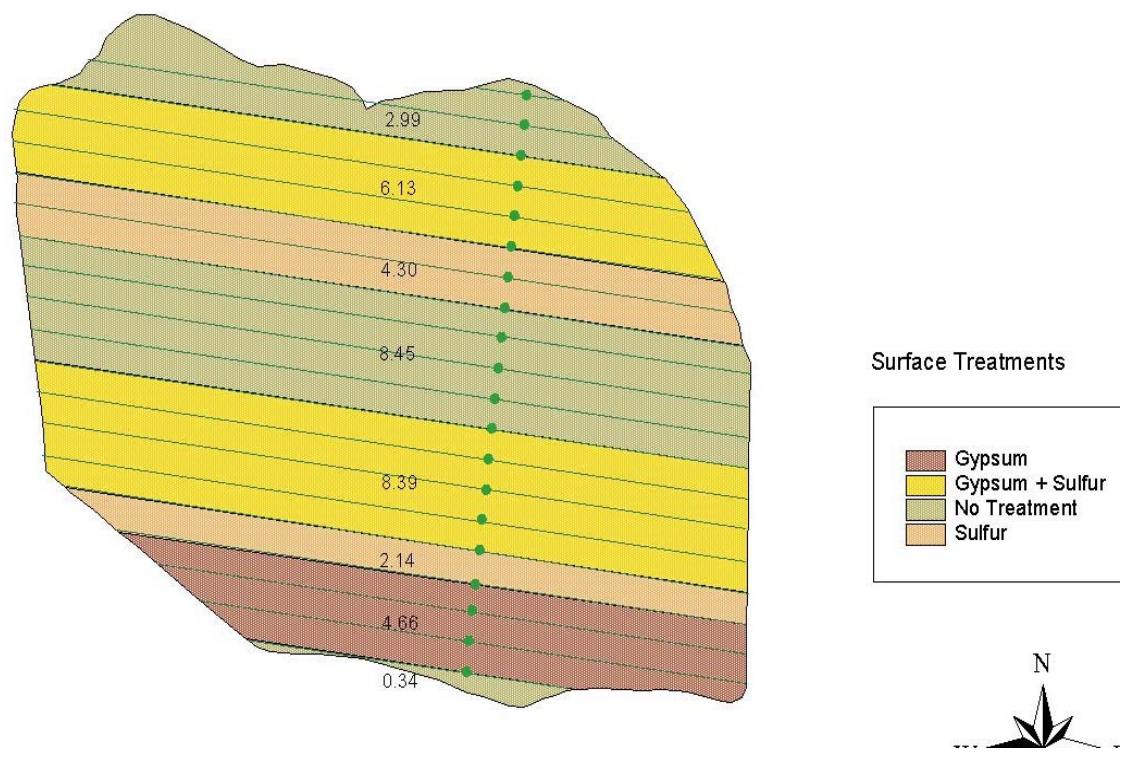

Figure 5. Location of soil amendments at the Cooksley Irrigation Study Site. Values = acreage.
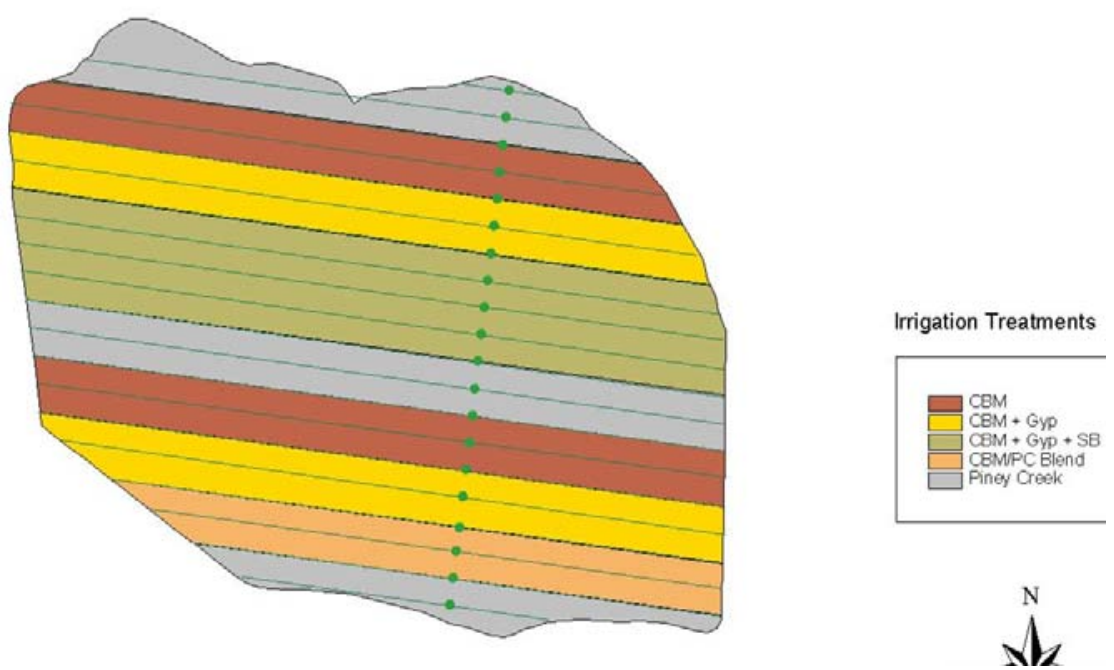

Figure 6. Locations of the CBM water treatments at the Cooksley Irrigation Study Site. 


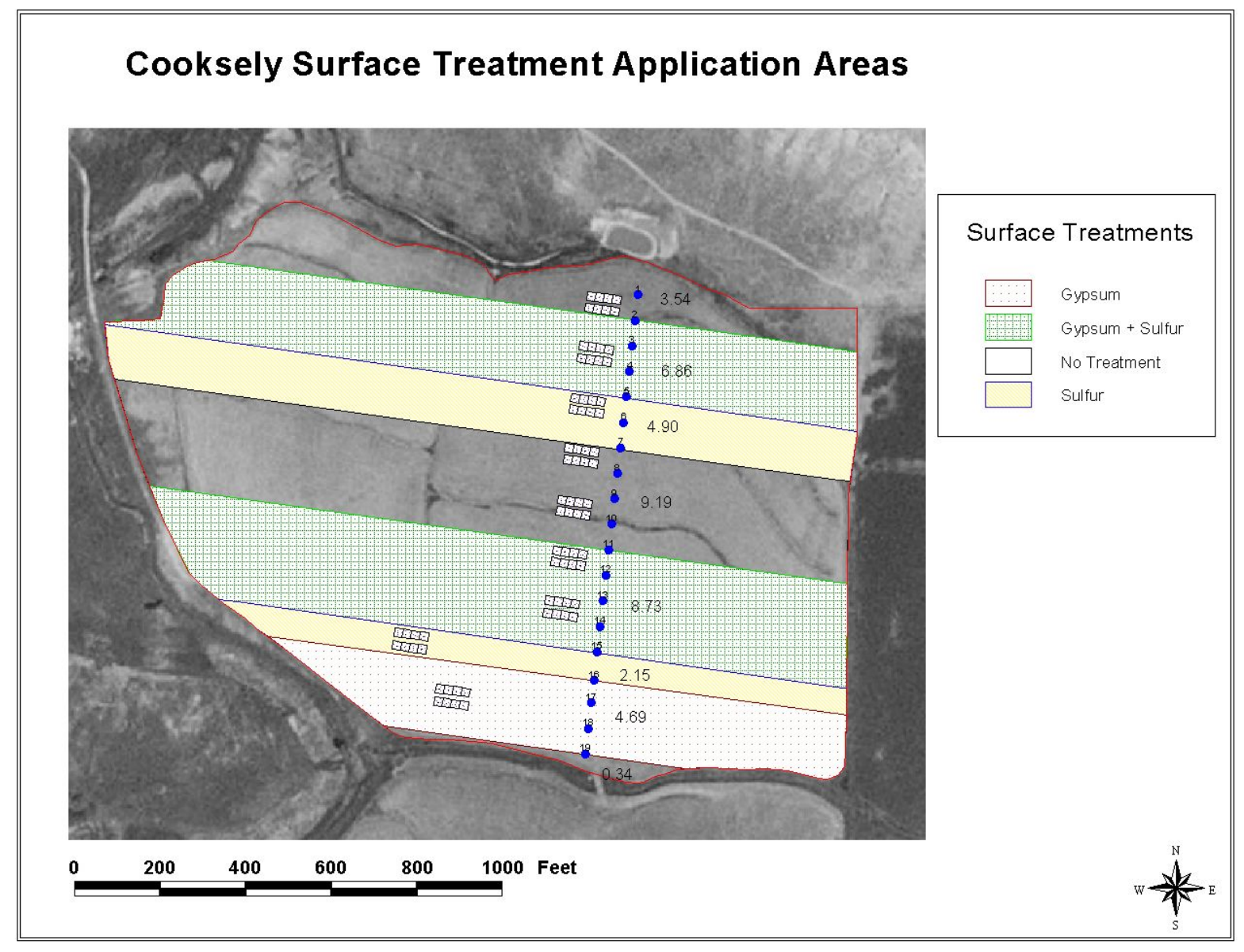

Figure 7. Soil study pit (rectangles) and riser locations (dots) at Cooksley irrigation site. Values represent acreage in each colored section.

Soil study plots were established throughout the Cooksley Ranch study area (Figure 7). Soil samples were collected from the top three soil horizons. Soil samples were homogenized and saturated paste extracts were prepared and analyzed for baseline $\mathrm{pH}, \mathrm{EC}, \mathrm{Ca}, \mathrm{Mg}, \mathrm{Na}, \mathrm{SAR}$, $\mathrm{SO}_{4}, \mathrm{HCO}_{3}$, and $\mathrm{CO}_{3}$. In addition, baseline soil hydraulic properties were determined, including saturated hydraulic conductivity using a tension infiltrometer and soil water retention function using gravimetric techniques. Soil chemical and physical properties were monitored at the beginning and end of each irrigation season.

Water samples were collected from CBM sources to be used for irrigation on the Cooksley Ranch study area and prepared and analyzed as described for water samples collected for the Column Studies.

A total of four water quality monitoring locations were established on Piney Creek and the irrigation ditch flowing east through the Cooksley Ranch property to monitor any impact the irrigation activities may have had on adjacent surface water resources. Monitoring locations are positioned upstream of the CBM irrigation research project and along the eastern boundary of the 
Cooksley Ranch. Baseline water samples were collected from the four locations and the samples were analyzed for $\mathrm{pH}$, EC, TDS, alkalinity, $\mathrm{Ca}, \mathrm{Mg}, \mathrm{Na}, \mathrm{K}, \mathrm{SO}_{4}, \mathrm{Cl}, \mathrm{CO}_{3}$, and $\mathrm{HCO}_{3}$.

\section{RESULTS AND DISCUSSION}

\section{Baseline Evaluations}

Detailed baseline evaluations were conducted at two potential research locations for the use of CBM produced water for managed irrigation. The first step in the baseline process was to map the soils at each site using a very detailed scale. The detailed soil survey including soil analysis for each of the study locations is presented in Appendix I. The results of the soil survey were used to determine the best site for the research project and the survey indicated that the Cooksley Ranch Study site was the preferred irrigation site primarily because of the texture of materials present at the site. However, in this case the final site determination was based on water availability rather than the baseline conditions characterizing the sites. The availability of water at the Leiter Ranch was questionable. Therefore, the Cooksley Ranch site became the official study site for the project.

\section{Column Experiments}

The CBM produced water quality data is shown in Table 2. The CBM water used in the laboratory study had a SAR of $24 \mathrm{mmol}^{1 / 2} \mathrm{~L}^{-1 / 2}$ and a relatively low EC of $1.4 \mu \mathrm{S} / \mathrm{cm}$. The quality of this water falls in the middle when compared to other CBM waters in the Powder River Basin. A more complete data list is presented in Appendix II.

The hydraulic conductivity relationships associated with the samples collected are presented in Figure 8 for the Leiter Ranch and a mixture of A horizon materials collected from several locations near the Cooksley Ranch. Data presented in Figure 8a is associated with the A horizon of the soil profile sampled at the Leiter Ranch. The data show virtually no difference in hydraulic conductivity $(\mathrm{K})$ values between the treated and untreated soils. Conversely, from midway through the experiment on, $\mathrm{K}$ is only slightly higher in the soils treated with gypsum (Figure 8b)

An additional leaching study was conducted using the soil materials collected from the Leiter Ranch Study site. In this study, the hydraulic conductivity of non-treated soil material and the hydraulic conductivity of soil treated with gypsum using CBM water was evaluated. As expected, the $\mathrm{K}$ of soil irrigated with water from Clear Creek was higher than the $\mathrm{K}$ of soil irrigated with CBM product water (Figure 9). The variability of the data from the soil cores that received treated $\mathrm{CBM}$ water was too large to allow us to distinguish a difference in the $\mathrm{K}$ of this treatment from the $\mathrm{K}$ of soil cores irrigated with either Clear Creek water or CBM water (data not shown graphically). Chemical analysis of soil core material is presented in Appendix III. 
Table 2. Baseline water quality analysis for surface and produced water sources at the Cooksley irrigation site.

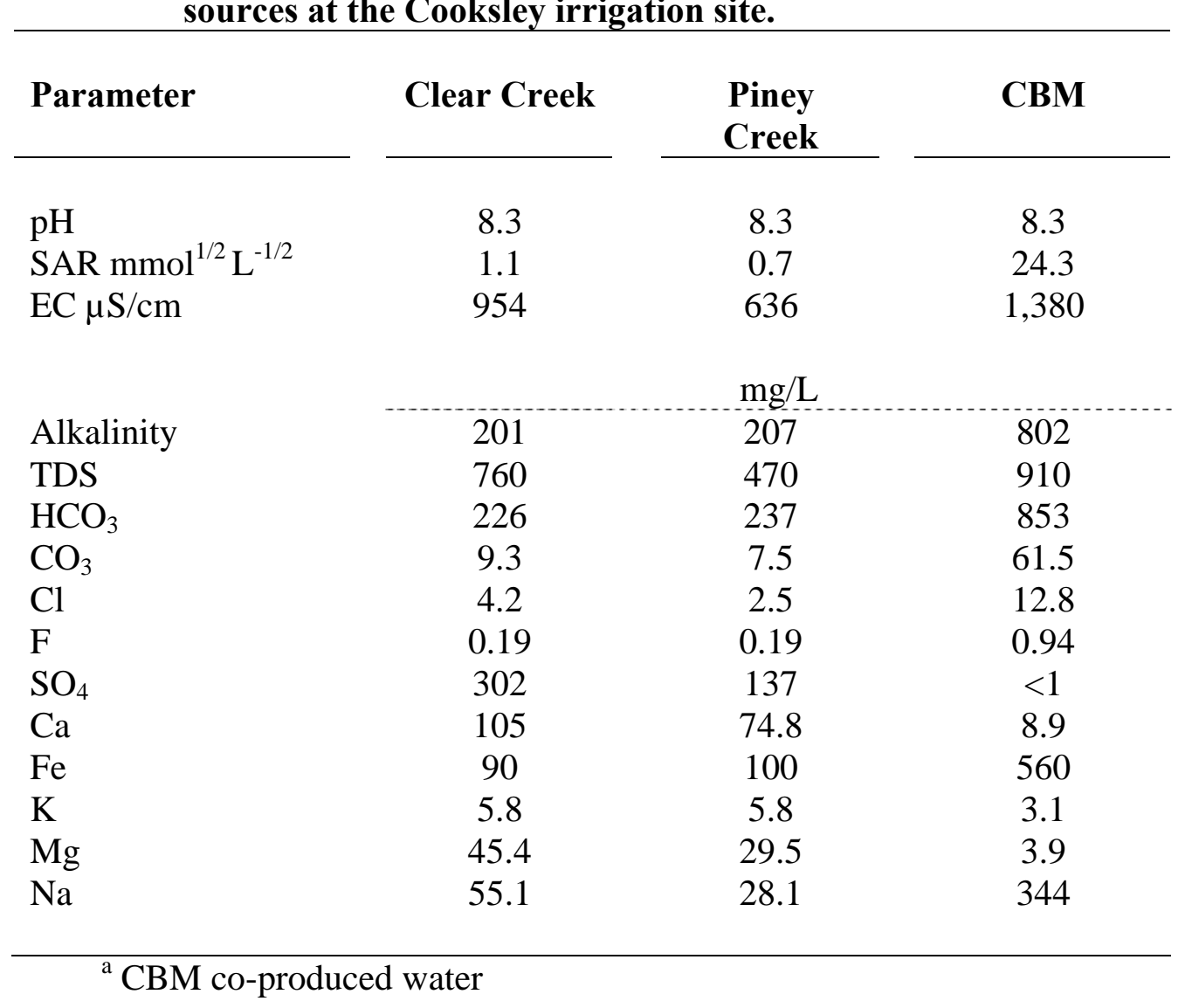
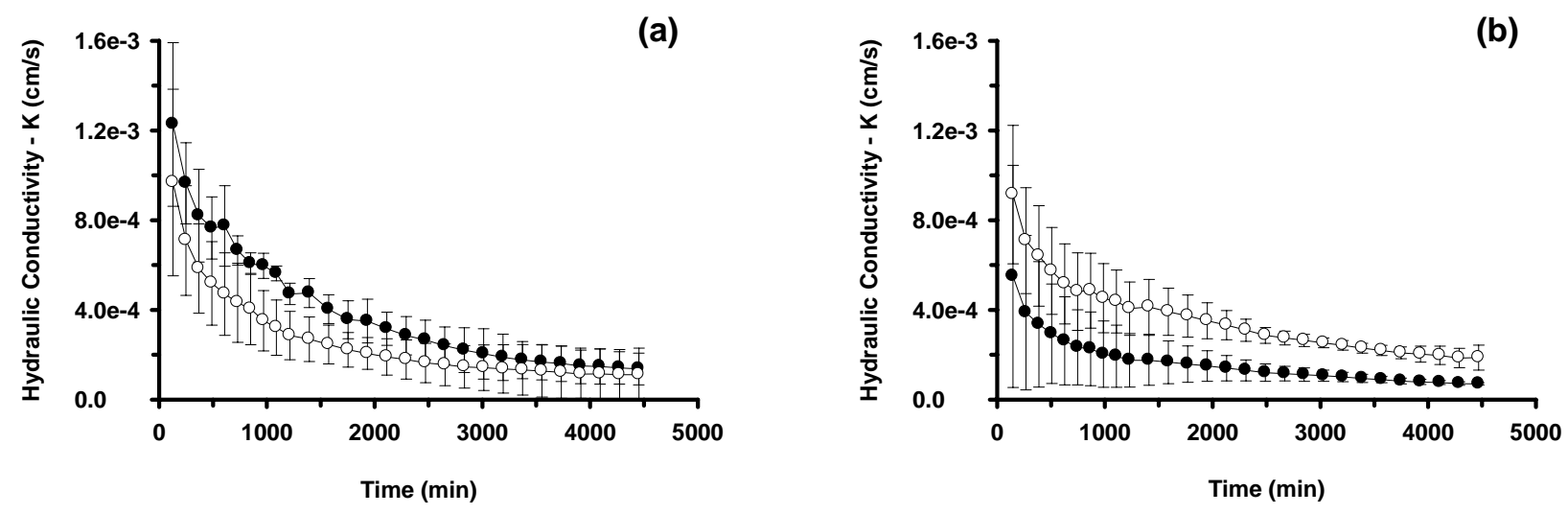

Figure 8. Hydraulic conductivity of the A horizon of soil material collected from the Leiter Ranch (a) and the Cooksley Ranch (b), near Buffalo, Wyoming, irrigated with CBM product water. Soil was either untreated $(\bullet)$ or treated with the equivalent of 2.5 tons of gypsum/acre ( $)$. Error bars are $95 \%$ confidence intervals $(n=4)$. 


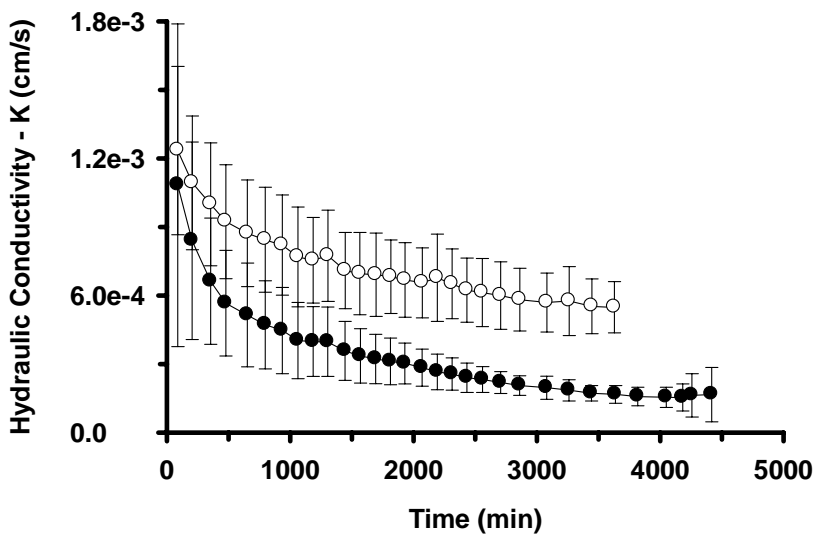

Figure 9. Hydraulic conductivity of Leiter Ranch soil material using CBM produced water $(\bullet)$ and Clear Creek water $(\odot)$. Error bars are $95 \%$ confidence intervals $(n=4)$.

Field Experiments

Conductivity values of the data collected using the tension infiltrometer were less variable than vales generated using the double ring infiltrometer. Therefore, the tension infiltrometer data was be used for comparisons between treatments in the field plots. The baseline data collected for each study plot are presented in Appendix IV.

In Fall 2003, the water treatment systems and side-roll irrigation system were installed and briefly tested before irrigation was terminated due to winter weather conditions. Gypsum and sulfur surface treatments were applied and approximately three inches of typical (Piney Creek) irrigation water was applied to the entire site to test the side-roll system during the first year. In addition, the CBM-PC Blend treatment was tested to ensure operation and control of water mixing. The water from the CBM-PC Blend testing was applied to the CBM-PC Blend plot area. Initial soil characterizations are presented in Appendix V.

The irrigation season of 2004 was hampered by the low availability of CBM produced water at the Cooksley Ranch irrigation study site. The available water allowed irrigation of the field plot site but did not allow the irrigation of the entire field. Although this was a set-back to the landowner, the research effort was not compromised and the irrigation was a success for the season. The infiltration evaluation was scheduled to be completed in the spring since frozen soils eliminated the opportunity to acquire the data in the fall, however, funding did not allow for completion of the infiltration study.

In Spring 2004, the field site was characterized prior to irrigation. Soil samples were collected from each plot for chemical analysis (Appendix VI).

Soil characterizations from Fall 2004 are presented in Appendix VII. Analyses indicated higher concentrations of sulfate in treatments where gypsum and sulfur were added to the 
irrigation water, as expected. The sulfur added to the irrigation water was in the sulfur dioxide form, which is easily converted to sulfate in the presence of oxygen. After the irrigation water was treated with sulfur dioxide, it was violently splashed into a holding tank, which aerated the water and allowed for the formation of sulfate in solution. Sulfate is an anion and, therefore, is expected to be leached from the system quickly. This is due, for the most part, to the fact that it does not interact with the cation exchange capacity of the soil. This was confirmed by the increase in sulfate in the Bt-2 horizon with no leached Na present. Surface applied sulfur is delayed in its activity and subsequent leaching because it depends on microbial transformation and oxidation to go from elemental sulfur to sulfate. This takes time and is an important reason why surface applied sulfur treatments lag behind sulfur added to irrigation water treatments. Results from the titration experiment indicate that a $\mathrm{pH}$ of 6.5 was optimal for the removal of bicarbonate.

Sodium concentrations increased substantially in the first two soil horizons (A and Bt-1) from Spring to Fall 2004 in all test plots except those receiving Piney Creek (PC) water and a blend of PC water and CBM water (Figures 10 and 11). The blend of PC and CBM water resulted in the highest $\mathrm{Na}$ concentrations in the third horizon (Bt-2), however, these values were highly variable and it is difficult to determine the cause of this from a single irrigation season (Figure 12). SAR values increased substantially from Spring to Fall 2004 in the A and Bt-1 horizons in all plots except those receiving PC water and a blend of PC and CBM water (Figures 13 and 14). SAR values in the Bt-2 horizon increased in plots receiving a blend of PC and CBM water from Spring to Fall 2004, however, as with the Na concentrations, interpretation of this data is hampered by high variability (Figure 15). The higher variability of the PC-CBM blend treatments is likely due to only using 2 replicates in these treatments rather than 4 , which was the number of replicates used in the other treatments.

When comparing the A horizon chemistry of the different treatments that were only irrigated with CBM water, it appears that no treatment to the soil or water resulted in the highest SAR $\left(7.8 \mathrm{mmol}^{1 / 2} \mathrm{~L}^{-1 / 2}\right)$, followed by a gypsum amendment to the soil $\left(5.6 \mathrm{mmol}^{1 / 2} \mathrm{~L}^{-1 / 2}\right)$, gypsum injection and sulfur burner treatment to the water $\left(5.5 \mathrm{mmol}^{1 / 2} \mathrm{~L}^{-1 / 2}\right)$, and a combination of a gypsum amendment to the soil and gypsum injection and sulfur burner treatment to the water (3.7 mmol ${ }^{1 / 2} \mathrm{~L}^{-1 / 2}$; Figure 16a). The benefit of using a gypsum amendment to the soil or gypsum injection and sulfur burner treatment to the water are indistinguishable when comparing SAR values (Figure 16a) and this is supported by the very similar Na and Ca concentrations in the soil following these two treatments (Figures 16b and 16c, respectively). The most affective treatment option appears to be a combination of these two treatment methods (1-gypsum amendment to the soil and 2-gypsum injection and sulfur burner treatment to the water), which decreased the SAR value by 53\% compared to using CBM water with no amendments (from 7.8 down to $3.7 \mathrm{mmol}^{1 / 2} \mathrm{~L}^{-1 / 2}$; Figure 16a). This large reduction in SAR is likely driven by the much higher $\mathrm{Ca}$ concentrations in the soil following this treatment combination compared to the other treatments (Figure 16c). 


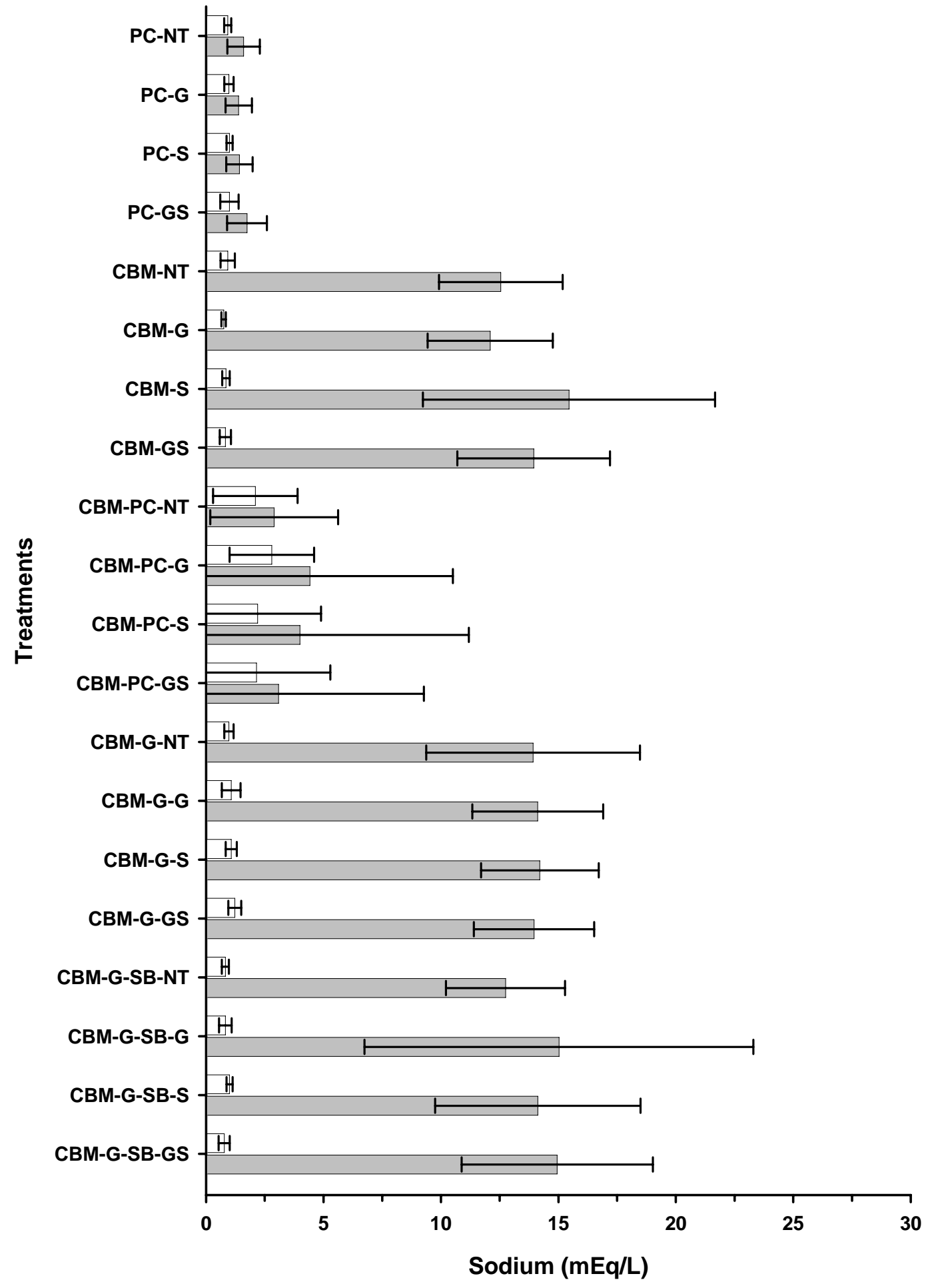

Figure 10. Sodium concentrations in the first soil horizon (A) at the Cooksley Ranch site in Spring 2004 (open bars) and Fall 2004 (shaded bars) before and after irrigation with water from Piney Creek and (or) CBM produced water with various soil and water amendments of gypsum and sulfur (Table 1). Error bars are $95 \%$ confidence intervals $(n=4$ for all treatments except CBM-PC-* blends where $n=2$ ). 


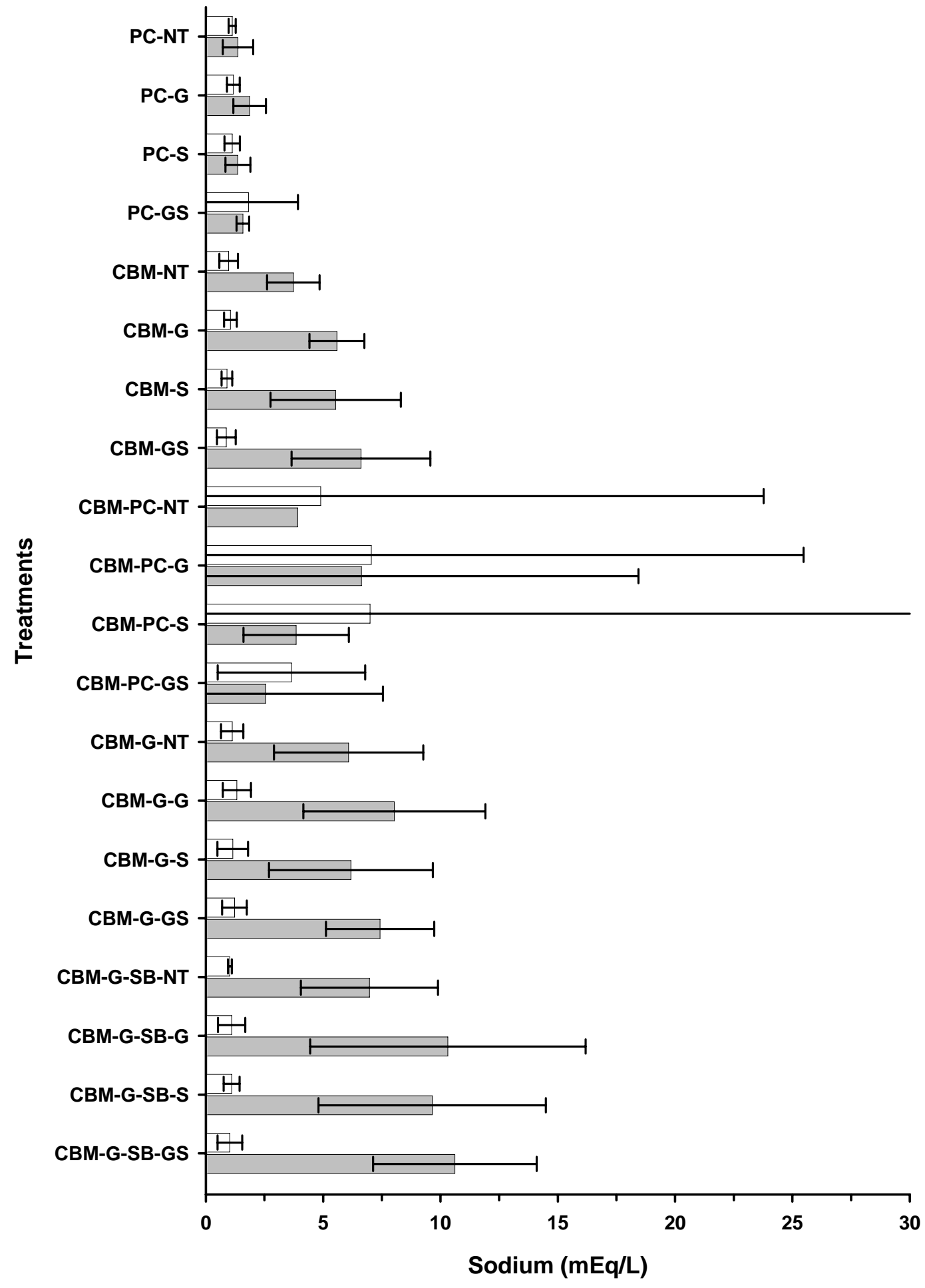

Figure 11. Sodium concentrations in the second soil horizon (Bt-1) at the Cooksley Ranch site in Spring 2004 (open bars) and Fall 2004 (shaded bars) before and after irrigation with water from Piney Creek and (or) CBM produced water with various soil and water amendments of gypsum and sulfur (Table 1). Error bars are $95 \%$ confidence intervals $(n=4$ for all treatments except CBM-PC-* blends where $n=2$ ). 


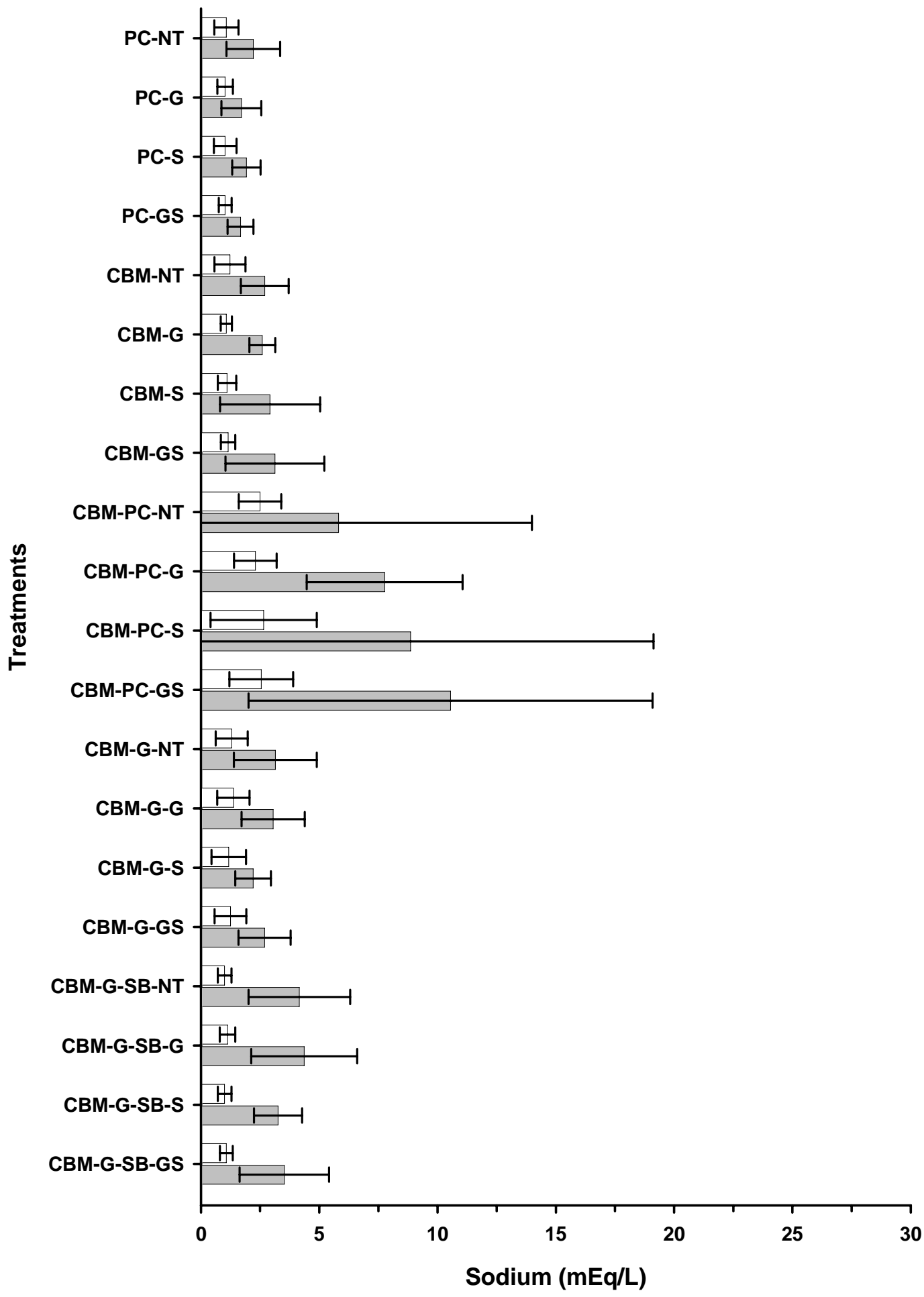

Figure 12. Sodium concentrations in the third soil horizon (Bt-2) at the Cooksley Ranch site in Spring 2004 (open bars) and Fall 2004 (shaded bars) before and after irrigation with water from Piney Creek and (or) CBM produced water with various soil and water amendments of gypsum and sulfur (Table 1). Error bars are $95 \%$ confidence intervals $(n=4$ for all treatments except CBM-PC-* blends where $n=2$ ). 


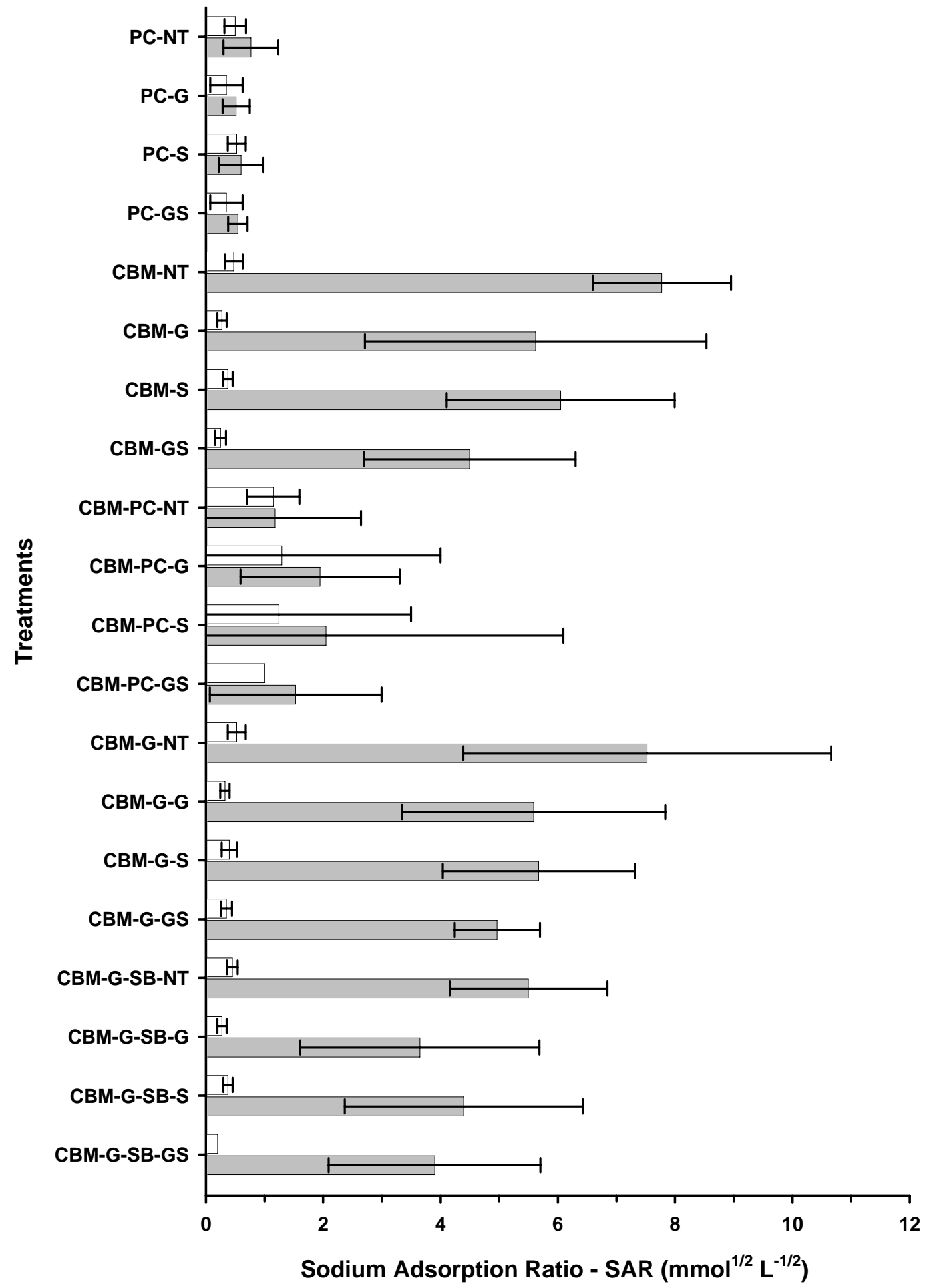

Figure 13. Sodium adsorption ratios (SAR) in the first soil horizon (A) at the Cooksley Ranch site in Spring 2004 (open bars) and Fall 2004 (shaded bars) before and after irrigation with water from Piney Creek and (or) CBM produced water with various soil and water amendments of gypsum and sulfur (Table 1). Error bars are $95 \%$ confidence intervals $(n=4$ for all treatments except CBMPC-* blends where $n=2$ ). 


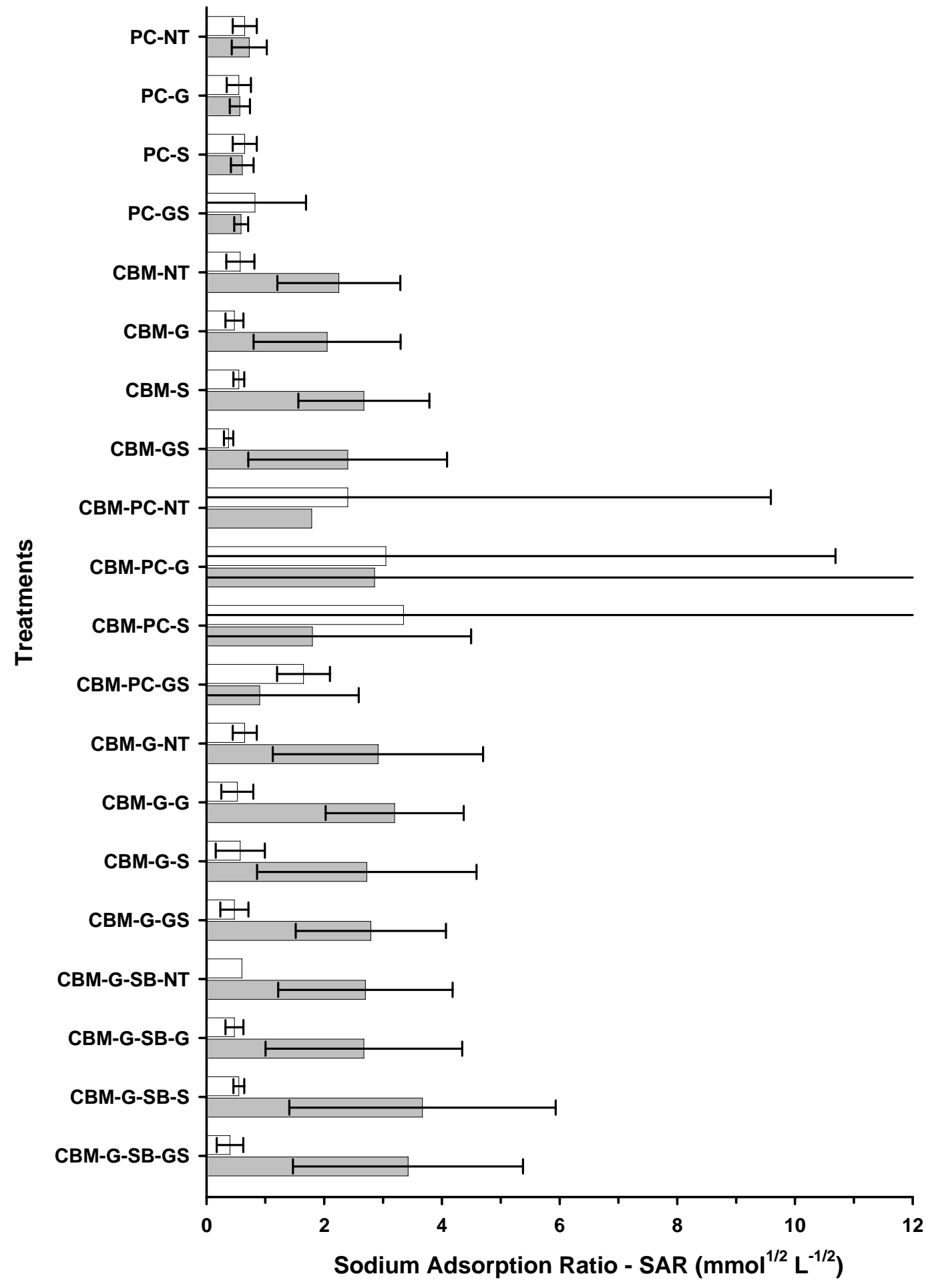

Figure 14. Sodium adsorption ratios (SAR) in the second soil horizon (Bt-1) at the Cooksley Ranch site in Spring 2004 (open bars) and Fall 2004 (shaded bars) before and after irrigation with water from Piney Creek and (or) CBM produced water with various soil and water amendments of gypsum and sulfur (Table 1). Error bars are $95 \%$ confidence intervals $(n=4$ for all treatments except CBM-PC-* blends where $n=2$ ). 


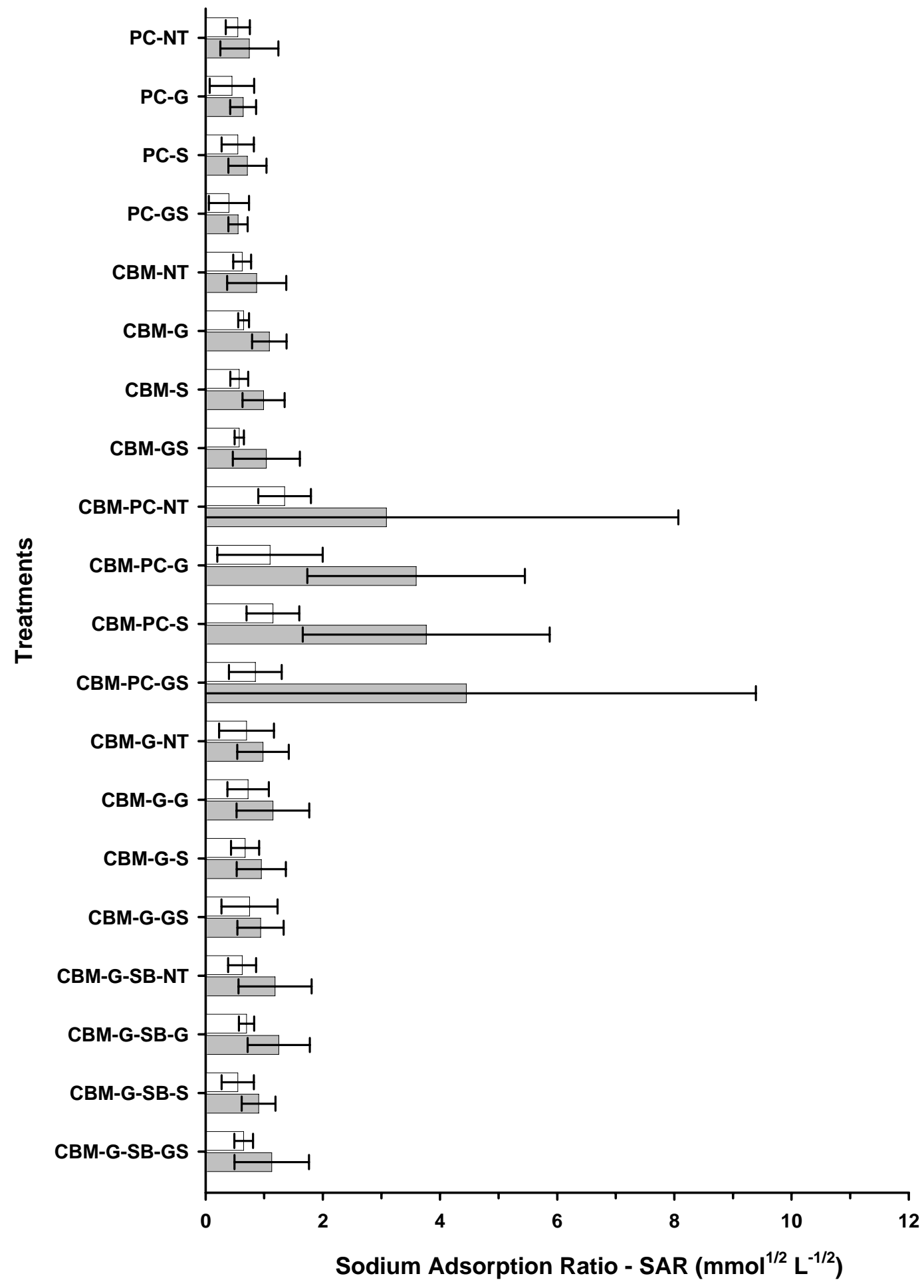

Figure 15. Sodium adsorption ratios (SAR) in the third soil horizon (Bt-2) at the Cooksley Ranch site in Spring 2004 (open bars) and Fall 2004 (shaded bars) before and after irrigation with water from Piney Creek and (or) CBM produced water with various soil and water amendments of gypsum and sulfur (Table 1). Error bars are $95 \%$ confidence intervals $(n=4$ for all treatments except CBMPC-* blends where $n=2$ ). 

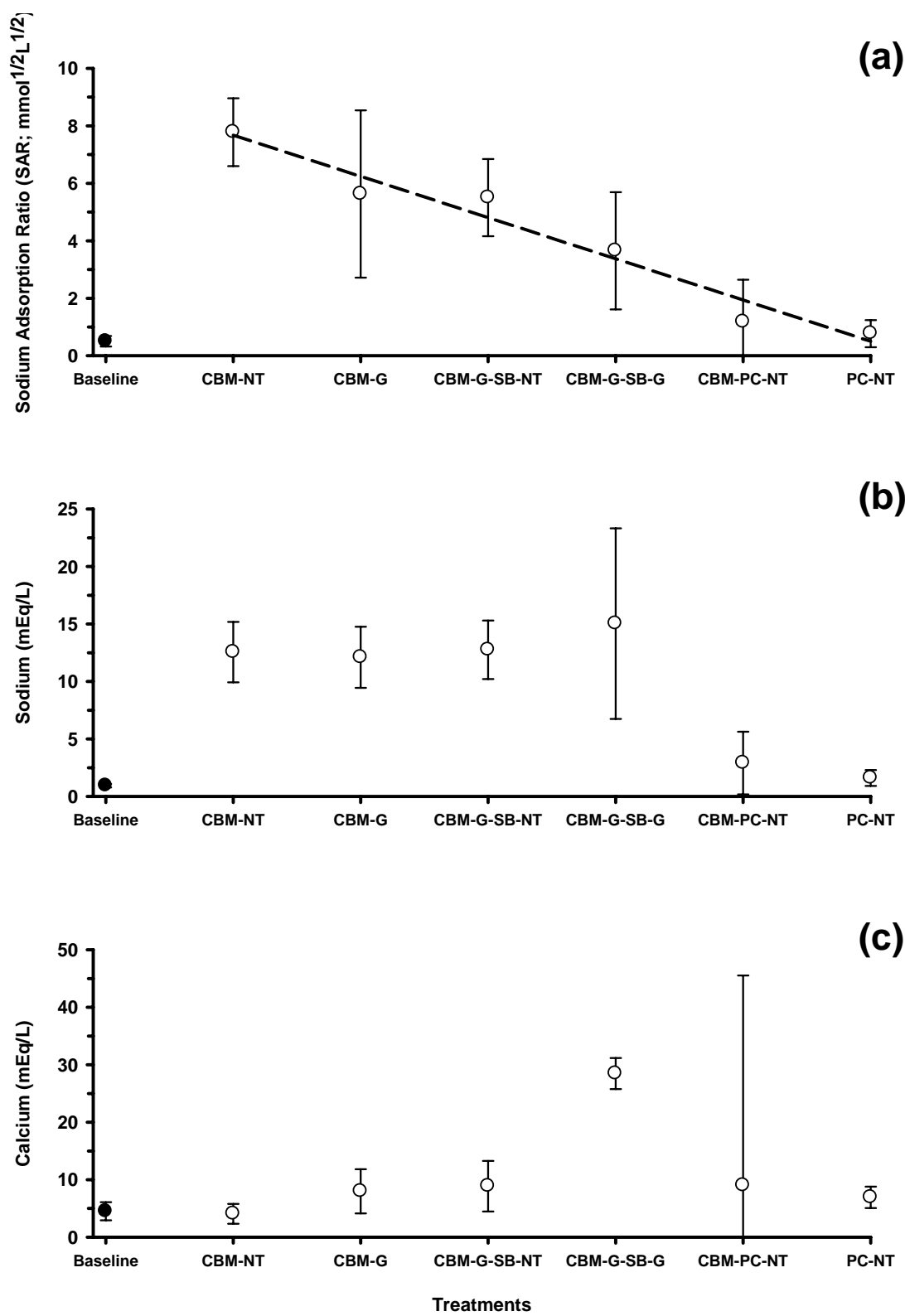

Figure 16. A comparison of the effects of certain soil and water treatment amendments (Table 1) on the soil chemistry at the Cooksley irrigation site following irrigation in Fall 2004 ( $)$ along with a Baseline value which is the soil chemistry in Spring 2004 from the plot area that received no soil or water amendments and was irrigated with Piney Creek water $(\bullet)$. Error bars are 95\% confidence intervals $(n=4$ for all treatments except CBM-PC-NT where $n=2)$. The regression line in panel "a" is meant only to show the data trend resulting from the different treatments. 


\section{CONCLUSIONS}

After a single irrigation season, using CBM produced water resulted in elevated Na and SAR values in the first two soil horizons. This problem did not occur when water from Piney Creek or a mixture (50:50) of Piney Creek water and CBM produced water was used for irrigation. High Na concentrations in the first and second soil horizons indicate the various treatments to the soil and irrigation water did not completely eliminate Na accumulation in the root zone, resulting in SAR values higher than baseline. Among the treatments tested, a combination of using a gypsum amendment to the soil along with a gypsum injection and sulfur burner treatment to the irrigation water resulted in the lowest SAR value in the first soil horizon among treatments irrigated solely with CBM produced water. The SAR value resulting from this combination treatment was 53\% lower than using CBM water with no amendments to soil or water and $\sim 33 \%$ lower than using (1) a gypsum amendment to the soil or (2) a gypsum injection and sulfur burner treatment to the water.

\section{REFERENCES}

Abu-Sharar, T.M., F.T. Bingham, and J.D. Rhodes. 1987. Stability of Soil Aggregates as Affected by Electrolyte Concentration and Composition. Soil Sci. Soc. Am. J. 51:309314.

Alperovitch, N., I. Shainberg, and R. Keren. 1981. Specific Effect of Magnesium on the Hydraulic Conductivity of Sodic Soils. J. Soil Sci. 32:543-554.

Arora, H.S., and N.T. Coleman. 1979. The Influence of Electrolyte Concentration on Flocculation of Clay Suspensions. Soil Sci. 127:134-139.

Cass, A., and M.E. Sumner. 1982. Soil Pore Structural Stability and Irrigation Water Quality: II. Sodium Stability Data. Soil Sci. Soc. Am. J. 46:507-512.

Chi, C.L., W.W. Emerson, and D.G. Lewis. 1977. Exchangeable Calcium, Magnesium and Sodium and the Dispersion of Illites in Water. I. Characterization of Illites and Exchange Reactions. Aust. J. Soil Res. 15:243-253.

Emerson, W.W., and C.L. Chi. 1977. Exchangeable Calcium, Magnesium and Sodium and the Dispersion of Illites in Water. II. Dispersion of Illites in Water. Aust. J. Soil Res. 15:255262.

Frenkel, H., J.O. Goertzen, and J.D. Rhodes. 1978. Effects of Clay Type and Content, Exchangeable Sodium Percentage, and Electrolyte Concentration on Clay Dispersion and Soil Hydraulic Conductivity. Soil Sci. Soc. Am. J. 48:32-39.

Gardner, W.R., M.S. Mayhugh, J.O. Goertzen, and C.A. Bower. 1959. Effect of Electrolyte Concentration and ESP on Diffusivity of Water in Soils. Soil Sci. 88:270-274.

Jayawardane, N.S. 1979. An Equivalent Salt Solutions Method for Predicting Hydraulic Conductivities of Soils for Different Salt Solutions. Aust. J. Soil Res. 17:423-428.

McNeal, B.L., and N.T. Coleman. 1966. Effect of Solution Composition on Soil Hydraulic Conductivity. Soil Sci. Soc. Am. Proc. 30:308-312. 
Pupisky, H., and I. Shainberg. 1979. Salt Effects on the Hydraulic Conductivity of a Sandy Soil. Soil Sci. Soc. Am. J. 43:429-433.

Quirk, J.P., and R.K. Schofield. 1955. The Effect of Electrolyte Concentration on Soil Permeability. J. Soil Sci. 6:163-178.

Rengasamy, P., and M. E. Sumner. 1998. Processes Involved in Sodic Soils. Pp. 35-50. In Malcolm E. Sumner and Ravendra Naidu (eds.), Sodic Soils: Distribution, Properties, Management, and Environmental Consequences. Oxford University Press, New York.

Rengasmy, P., and K.A. Olsson. 1991. Irrigation and Sodicity. Aust. J. Soil Res. 31:821-837.

Rhodes, J.D. 1972. Quality of Irrigation Water. Soil Sci. 113:277-284.

Rhodes, J.D., and R.D. Ingvalson. 1969. Macroscipic Swelling and Hydraulic Conductivity Properties of Four Vermiculite Soils. Soil Sci. Soc. Am. Proc. 33:364-369.

RIENR (Ruckelshaus Institute of Environment and Natural Resources). 2005. Water Production from Coalbed Methane Development in Wyoming: A summary of Quantity, Quality and Management Options. University of Wyoming, www.uwyo.edu/enr/ienr/cbm.asp.

Russo, D., and E. Bresler. 1977. Analysis of the Saturated and Unsaturated Hydraulic Conductivity in Mixed Sodium and Calcium Soil Systems. Soil Sci. Soc. Am. J. 41:706712.

Shainberg, I., G.J. Levy, P. Rengasamyu, and H. Frenkel. 1992. Aggregate Stability and Seal Formation as Affected by Drops' Impact Energy and Soil Amendments. Soil Sci. 154:113-119.

Shanmuganathan, R.T., and J. M. Oades. 1983. Modification of Soil Physical Properties by Addition of Calcium Compounds. Aust. J. Soil Res. 21:285-300.

Slade, P.G., and J.P. Quirk. 1991. The Limited Crystalline Swelling of Smectite in $\mathrm{CaCl}_{2}$, $\mathrm{MgCl}_{2}$, and $\mathrm{LaCl}_{2}$ Solutions. J. Colloid Interface Sci. 144:18-26.

Sposito, G. 1989. The Chemistry of Soils. Oxford University Press, New York, NY.

Sumner, M.E., P. Rengasamy and R. Naidu. 1998. Sodic Soils: A Reappraisal. Pp. 3-17. In Malcolm E. Sumner and Ravendra Naidu (eds.), Sodic Soils: Distribution, Properties, Management, and Environmental Consequences. Oxford University Press, New York.

USSL (United States Salinity Laboratory). 1954. Diagnosis and Improvement of Saline and Alkali Soils. USDA, U.S. Govt. Printing Office, Washington, D.C.

Van Olphen, H. 1977. An Introduction to Clay Colloid Chemistry. $2^{\text {nd }}$ Ed., John Wiley, New York, NY.

Waldron, L.J., and G.K. Constantin. 1970. Soil Hydraulic Conductivity and Bulk Volume Changes During Cyclic Calcium-Sodium Exchange. Soil Sci. 110:81-85.

Yousaf, M., O.M. Ali, and J.D. Rhodes. 1987. Dispersion of Clay From Some Salt-Affected, Arid Land Soil Aggregates. Soil Sci. Soc. Am. J. 56:1257-1260. 


\section{Appendix I}

\section{Soil Survey of Coal Bed Methane Product Water Application Areas at the Cooksley Ranch and Leiter Ranch Study Sites}




\section{Introduction}

Wolverine Operations selected a 52.6 ac field near Leiter, Wyoming and a 37.5 ac field along Piney Creek, Wyoming, the Cooksley Ranch, for irrigation with coal bed methane (CBM) co-produced water. Historically, these areas have been cultivated and used for grazing and harvesting of hay grass. A soil survey was completed by the Western Research Institute (WRI) to evaluate the extent and proportion and to document the physicochemical properties of the major soil types within each area and to asses their suitability for irrigation with CBM product water. Preliminary analyses of soil chemistry and soil texture have been completed by WRI and are included with this report.

\section{Methods}

The Leiter and Cooksley fields were traversed by a soil scientist and test-pits were randomly located in each area (Exhibits 1 and 2, respectively). The location of all test sites was recorded using a global positioning system (GPS). At each site a test-pit was excavated to a depth of approximately 48 inches and the soil profile was described. Descriptions included depth, texture-by-feel, consistence, structure, effervescence, percentage of coarse fragments, and visible salts for each soil horizon. Also noted were soil type, slope, aspect, and any other characteristics pertinent to pedon classification or suitability determinations.

Soil samples were collected from randomly selected soil profiles. Soil samples were analyzed by WRI for $\mathrm{pH}$ and electrical conductivity (EC), and texture with percent sand, silt, and clay. Saturated paste extracts were submitted to Green Analytical., Durango Colorado for analysis of calcium, magnesium, sodium, potassium, sodium adsorption ratio (SAR), alkalinity, chloride, and sulfate.

Soil boundaries were drawn on an orthogonal photograph base-map to reflect field observations as test-sites were described. After mapping was complete, the revised soil boundaries were digitized and soil maps were prepared at a scale of 1:3600 using ARCVIEW.

\section{Results}

A soil survey was conducted for the 52.6 ac Leiter Field \#1 and the 37.5 ac Cooksley Ranch Study Site in Sheridan County, Wyoming. The purpose of this survey was to identify the extent and proportion of soils in each area and to determine their suitability for application of CBM product water. The soils in both areas formed predominately in mixed alluvium from sedimentary rock sources. The following are the soil-mapping units identified in each area: 
Leiter Field \#1

- Forkwood loam

- Hiland fine sandy loam

Cooksley Ranch Study Site

- Forkwood silty clay loam

- Kishona loam

- Ulm silty clay loam

The extent and proportion of soils in the Leiter Field \#1 and Cooksley Ranch Study Site are shown on Exhibits 1 and 2, respectively. Soil Profile descriptions are provided for test-pit locations in area (Appendices $\mathrm{A}$ and $\mathrm{B}$, respectively). The A-horizon and Bt-horizons were selected for sampling from four test-pit locations in the Leiter Field \#1 and one test-pit in the Cooksley Ranch Study Site. The results from the laboratory analyses for the Leiter and CR fields are provided in Tables 1 and 2, respectively. Analysis of extractable cations, hydraulic conductivity, and soil texture are being conducted by WRI. A supplementary report of laboratory results and data interpretation will be submitted upon completion. Additional information regarding the physical and chemical properties of soils within the Leiter and CR fields was summarized from the Soil Survey of Sheridan County, Wyoming (USDA-NRCS, 1986) (Table 3).

The Leiter Field \#1 is composed of two soil mapping units: Forkwood loam and Hiland fine sandy loam. The predominant soil type identified in the Leiter Field is Forkwood which represents $97 \%$ (51.2 ac) of the survey area. The minor soil type located in the southwestern part of the survey area is Hiland. This map unit represents 3\% $(1.4 \mathrm{ac})$ of the survey area. The Hiland soil does not have limitations for irrigation. The primary limitations for irrigation in the Leiter Field are clay content in the surface horizons and platy structure of the A-horizon associated with the Forkwood mapping unit. Fine-textured materials have greater potential for clay dispersion and aggregate dispersion than coarser-textured soils. Investigation of the Leiter Field shows a platy structure of the A-horizon (surface) largely throughout the Forkwood mapping unit. The formation of platy structure is likely the result of compaction from high intensity grazing and rain-drop impacts to the soil surface when low vegetation cover is present. In addition, the Leiter Field contains a high density of annual plants that may proliferate under an irrigated regime. Cultivation and replanting of the Leiter Field may be warranted to ameliorate the compacted surface condition and presence of annual species.

The Cooksley Ranch Study Site is composed of three soil mapping units: Forkwood silty clay loam, Kishona loam, and Ulm silty clay loam. Forkwood silty clay loam is the predominant map unit and represents 83 \% (31 ac) of the survey area. Kishona and Ulm soils make up 5\% (2 
ac) $12 \%$ (4.5 ac) of the survey area. The Ulm mapping unit in the Cooksley Ranch Study Site represents the greatest limitations to this site because of elevated clay concentrations throughout the profile. Clay content in the surface horizons ranges from 28 to $50 \%$ and will typically result in a low permeability condition. Clay content of the Forkwood and Kishona soils ranges from 20 to $35 \%$, but these soils are structurally more stable than their counterparts in the Leiter Field. This is largely because of the management practices that have been employed at the two sites.

\section{Conclusion}

Field investigation and laboratory analysis show that soils in the Leiter Field may have limitations for irrigation due to clay content and compacted surface zones. Cultivation of this site may improve surface conditions for infiltration. The Cooksley Ranch site has few limitations for irrigation, however low permeability conditions may exist in the Ulm mapping unit. Hydraulic conductivity of these materials is being evaluated and will be presented upon completion. 
Table 1. Leiter Field \#1 - Results from laboratory analysis of soil samples collected from the Leiter Ranch Study site.

\begin{tabular}{|c|c|c|c|c|c|c|c|c|}
\hline LOCATION & $\begin{array}{l}\text { SAMPLE } \\
\text { DATE }\end{array}$ & $\begin{array}{l}\text { SAMPLE } \\
\text { DEPTH }\end{array}$ & PH UNITS & ЕC MMHO/СM & $\%$ SAT & $\begin{array}{l}\text { CALCIUM } \\
\text { MEQ/L }\end{array}$ & $\begin{array}{l}\text { MAGNESIUM } \\
\text { MEQ/L }\end{array}$ & $\begin{array}{c}\text { SODIUM } \\
\text { MEQ/L }\end{array}$ \\
\hline LEITER 1-1 & 04/03/03 & $0-7 "$ & 8.51 & 0.57 & 60.57 & 3.76 & 2.53 & 0.45 \\
\hline LEITER 1-1 DUP & 04/03/03 & $0-7 "$ & 8.76 & 0.59 & 45.60 & 3.63 & 2.48 & 0.67 \\
\hline LEITER 1-2 & 04/03/03 & 7-15" & 8.93 & 0.80 & 36.03 & 5.24 & 3.33 & 2.02 \\
\hline LEITER 1-3 & 04/03/03 & $15-30 "$ & 8.94 & 0.64 & 39.16 & 2.48 & 1.58 & 3.50 \\
\hline LEITER 2-1 & 04/03/03 & $0-5 "$ & 8.31 & 0.67 & 40.40 & 3.85 & 2.75 & 0.84 \\
\hline LEITER 2-2 & 04/03/03 & 5-15" & 8.74 & 0.50 & 45.50 & 3.11 & 1.99 & 1.06 \\
\hline LEITER 2-3 & 04/03/03 & $15-30 "$ & 8.93 & 0.71 & 52.23 & 3.30 & 2.10 & 3.17 \\
\hline LEITER 3-1 & 04/03/03 & $0-6 "$ & 8.66 & 0.63 & 35.50 & 4.15 & 2.39 & 1.26 \\
\hline LEITER 3-2 & 04/03/03 & 6-18" & 8.74 & 0.36 & 44.53 & 1.54 & 0.99 & 1.60 \\
\hline LEITER 3-2 DUP & 04/03/03 & 6-18" & 8.5 & 0.38 & 44.36 & 1.53 & 1.00 & 1.71 \\
\hline LEITER 4-1 & 04/03/03 & $0-6 "$ & 7.97 & 0.69 & . & 3.28 & 2.65 & 1.17 \\
\hline LEITER 4-2 & 04/03/03 & 6-12" & 8.62 & 0.47 & 31.33 & 1.85 & 1.52 & 1.86 \\
\hline LEITER 4-3 & 04/03/03 & 12-24" & 8.97 & 0.76 & 42.86 & 1.41 & 1.25 & 3.50 \\
\hline
\end{tabular}


Table 1 cont'd. Leiter Field \#1 - Results from laboratory analysis of soil samples collected from the Leiter Ranch Study site.

\begin{tabular}{cccccccc} 
LOCATION & $\begin{array}{c}\text { SAMPLE } \\
\text { DATE }\end{array}$ & $\begin{array}{c}\text { SAMPLE } \\
\text { DEPTH }\end{array}$ & SAR & \% SAND & \% SILT & \% CLAY & CLASS \\
\hline \hline LEITER 1-1 & $04 / 03 / 03$ & $0-7 "$ & 0.25 & 18.7 & 44.6 & 36.7 & SICL \\
LEITER 1-1 DUP & $04 / 03 / 03$ & $0-7 "$ & 0.38 &. &. &. & . \\
LEITER 1-2 & $04 / 03 / 03$ & $7-15 "$ & 0.97 & 35.7 & 38.6 & 25.7 & L \\
LEITER 1-3 & $04 / 03 / 03$ & $15-30 "$ & 2.46 & 22.6 & 43.8 & 33.5 & CL \\
LEITER 2-1 & $04 / 03 / 03$ & $0-5 "$ & 0.46 & 29.2 & 39.3 & 31.5 & CL \\
LEITER 2-2 & $04 / 03 / 03$ & $5-15 "$ & 0.66 & 32.7 & 38.8 & 28.5 & CL \\
LEITER 2-3 & $04 / 03 / 03$ & $15-30 "$ & 1.93 & 24.9 & 44.0 & 31.1 & CL \\
LEITER 3-1 & $04 / 03 / 03$ & $0-6 "$ & 0.70 & 53.4 & 25.9 & 20.7 & SCL \\
LEITER 3-2 & $04 / 03 / 03$ & $6-18 "$ & 1.42 & 35.6 & 28.3 & 36.1 & CL \\
LEITER 3-2 DUP & $04 / 03 / 03$ & $6-18 "$ & 1.52 &. &. & 3.9 & . \\
LEITER 4-1 & $04 / 03 / 03$ & $0-6 "$ & 0.68 & 40.2 & 33.8 & 26.0 & L \\
LEITER 4-2 & $04 / 03 / 03$ & $6-12 "$ & 1.43 & 38.8 & 38.2 & 22.9 & L \\
LEITER 4-3 & $04 / 03 / 03$ & $12-24 "$ & 3.03 & 32.5 & 31.1 & 36.3 & CL
\end{tabular}


Table 1 cont'd. Leiter Field \#1 - Results from laboratory analysis of soil samples collected from the Leiter Ranch Study site.

\begin{tabular}{cccccccc} 
LOCATION & $\begin{array}{c}\text { SAMPLE } \\
\text { DATE }\end{array}$ & $\begin{array}{c}\text { SAMPLE } \\
\text { DEPTH }\end{array}$ & $\begin{array}{c}\text { BICARBONATE } \\
\text { MEQ/L }\end{array}$ & $\begin{array}{c}\text { CARBONATE } \\
\text { MEQ/L }\end{array}$ & $\begin{array}{c}\text { CHLORIDE } \\
\text { MEQ/L }\end{array}$ & $\begin{array}{c}\text { SULFATE } \\
\text { MEQ/L }\end{array}$ & $\begin{array}{c}\text { POTASSIUM } \\
\text { MEQ/L }\end{array}$ \\
\hline \hline LEITER 1-1 & $04 / 03 / 03$ & $0-7 "$ & 3.6 & 0.1 & 0.59 & 0.67 & 0.38 \\
LEITER 1-1 DUP & $04 / 03 / 03$ & $0-7 "$ & 3.3 & 0.1 & 0.93 & 0.65 & 0.40 \\
LEITER 1-2 & $04 / 03 / 03$ & $7-15 "$ & 6.0 & 0.2 & 0.62 & 1.17 & 0.31 \\
LEITER 1-3 & $04 / 03 / 03$ & $15-30 "$ & 3.2 & 0.1 & 0.51 & 3.00 & 0.09 \\
LEITER 2-1 & $04 / 03 / 03$ & $0-5 "$ & 2.6 & 0.1 & 1.07 & 1.12 & 0.29 \\
LEITER 2-2 & $04 / 03 / 03$ & $5-15 "$ & 3.7 & 0.1 & 0.39 & $<0.10$ & 0.12 \\
LEITER 2-3 & $04 / 03 / 03$ & $15-30 "$ & 4.1 & 0.1 & 1.44 & 1.54 & 0.10 \\
LEITER 3-1 & $04 / 03 / 03$ & $0-6 "$ & 5.3 & 0.0 & 0.82 & 0.75 & 0.38 \\
LEITER 3-2 & $04 / 03 / 03$ & $6-18 "$ & 1.7 & 0.0 & 0.65 & 1.12 & $<0.10$ \\
LEITER 3-2 DUP & $04 / 03 / 03$ & $6-18 "$ & 1.7 & 0.0 & 0.54 & 1.17 & $<0.10$ \\
LEITER 4-1 & $04 / 03 / 03$ & $0-6 "$ & 1.6 & 0.0 & 1.24 & 1.17 & 0.35 \\
LEITER 4-2 & $04 / 03 / 03$ & $6-12 "$ & 2.0 & 0.0 & 1.13 & 0.71 & 0.11 \\
LEITER 4-3 & $04 / 03 / 03$ & $12-24 "$ & 1.9 & 0.0 & 0.96 & 0.54 & $<0.10$
\end{tabular}


Table 2. Cooksely Ranch Study Site - Results from laboratory analysis of soil samples collected from the Cooksley Ranch Study site.

\begin{tabular}{ccccccccc} 
LOCATION & $\begin{array}{c}\text { SAMPLE } \\
\text { DATE }\end{array}$ & $\begin{array}{c}\text { SAMPLE } \\
\text { DEPTH }\end{array}$ & PH UNITS & EC MMHO/CM & \% SAT & $\begin{array}{c}\text { CALCIUM } \\
\text { MEQ/L }\end{array}$ & $\begin{array}{c}\text { MAGNESIUM } \\
\text { MEQ/L }\end{array}$ & $\begin{array}{c}\text { SODIUM } \\
\text { MEQ/L }\end{array}$ \\
\hline \hline CR 1-1-1 & $04 / 03 / 03$ & $0-8 "$ & 8.77 & 0.86 & 49.91 & 6.64 & 2.47 & 1.91 \\
CR 1-1-2 & $04 / 03 / 03$ & $8-20 "$ & 8.66 & 0.61 & 40.25 & 4.12 & 1.94 & 1.29
\end{tabular}

\begin{tabular}{cccccccc} 
LOCATION & $\begin{array}{c}\text { SAMPLE } \\
\text { DATE }\end{array}$ & $\begin{array}{c}\text { SAMPLE } \\
\text { DEPTH }\end{array}$ & SAR & \% SAND & \% SILT & \% CLAY & CLASS \\
\hline \hline CR 1-1-1 & $04 / 03 / 03$ & $0-8 "$ & 0.89 & 19.7 & 49.8 & 30.4 & SICL \\
CR $1-1-2$ & $04 / 03 / 03$ & $8-20 "$ & 0.74 & 20.9 & 53.6 & 25.5 & SIL
\end{tabular}

\begin{tabular}{cccccccc} 
LOCATION & $\begin{array}{c}\text { SAMPLE } \\
\text { DATE }\end{array}$ & $\begin{array}{c}\text { SAMPLE } \\
\text { DEPTH }\end{array}$ & $\begin{array}{c}\text { BICARBONATE } \\
\text { MEQ/L }\end{array}$ & $\begin{array}{c}\text { CARBONATE } \\
\text { MEQ/L }\end{array}$ & $\begin{array}{c}\text { CHLORIDE } \\
\text { MEQ/L }\end{array}$ & $\begin{array}{c}\text { SULFATE } \\
\text { MEQ/L }\end{array}$ & $\begin{array}{c}\text { POTASSIUM } \\
\text { MEQ/L }\end{array}$ \\
\hline \hline CR $1-1-1$ & $04 / 03 / 03$ & $0-8 "$ & 6.8 & 0.4 & 0.68 & 2.81 & 0.48 \\
CR $1-1-2$ & $04 / 03 / 03$ & $8-20 "$ & 3.3 & 0.2 & 0.82 & 1.92 & 0.16
\end{tabular}


Table 3. Physical and chemical properties of Leiter Field \#1 and Cooksley Ranch soils ${ }^{1}$.

\begin{tabular}{|c|c|c|c|c|c|c|c|c|c|c|}
\hline Soil Series & Horizon & $\begin{array}{l}\text { Depth } \\
\text { in }\end{array}$ & $\begin{array}{l}\text { Hydro. } \\
\text { group }\end{array}$ & $\begin{array}{c}\text { Clay } \\
\%\end{array}$ & $\begin{array}{c}\text { Moist } \mathrm{P}_{\mathrm{b}} \\
\mathrm{g} / \mathrm{cm}^{3}\end{array}$ & $\begin{array}{c}\text { Permeability } \\
\text { in } / \mathrm{hr}\end{array}$ & $\begin{array}{c}\text { Avialable } \\
\text { Water Capacity }\end{array}$ & $\begin{array}{c}\text { Soil reacton } \\
\text { pH }\end{array}$ & $\begin{array}{c}\text { Salinity } \\
\text { mmhos/cm }\end{array}$ & $\begin{array}{c}\text { Shrink/swell } \\
\text { potential }\end{array}$ \\
\hline \multirow[t]{5}{*}{ Hiland } & A & $0-2$ & B & $8-18$ & $1.25-1.35$ & $2.0-6.0$ & $0.10-0.13$ & $6.6-7.8$ & $<2$ & Low \\
\hline & Bt1 & $2-8$ & & $20-35$ & $1.25-1.35$ & $0.6-2.0$ & $0.14-0.16$ & $7.4-7.8$ & $<2$ & Moderate \\
\hline & Bt2 & $8-26$ & & $20-35$ & $1.25-1.35$ & $0.6-2.0$ & $0.14-0.16$ & 7.9-9.0 & $<2$ & Low \\
\hline & Bt3 & $26-35$ & & $10-20$ & $1.35-1.45$ & $2.0-6.0$ & $0.10-0.14$ & 7.9-9.0 & $<2$ & Low \\
\hline & $\mathrm{Bk}$ & $35-48$ & & $10-20$ & $1.35-1.45$ & $2.0-6.0$ & $0.10-0.14$ & 7.9-9.0 & $<2$ & Low \\
\hline \multirow[t]{6}{*}{ Forkwood } & A & $0-4$ & B & $12-25$ & $1.15-1.25$ & $0.6-2.0$ & $0.15-0.17$ & $6.6-7.8$ & $<2$ & Low \\
\hline & Bt1 & 4-15 & & $25-35$ & $1.25-1.35$ & $0.6-2.0$ & $0.19-0.21$ & $6.6-7.8$ & $<2$ & Moderate \\
\hline & Bt2 & $15-25$ & & $20-32$ & $1.25-1.35$ & $0.6-2.0$ & $0.16-0.18$ & 7.9-9.0 & $<2$ & Low \\
\hline & Btk & $25-31$ & & $20-32$ & $1.25-1.35$ & $0.6-2.0$ & $0.16-0.18$ & 7.9-9.0 & $<2$ & Low \\
\hline & $\mathrm{Bk}$ & $31-44$ & & $20-32$ & $1.25-1.35$ & $0.6-2.0$ & $0.16-0.18$ & 7.9-9.0 & $<2$ & Low \\
\hline & Bky/Cky & $44-60$ & & $20-32$ & $1.25-1.35$ & $0.6-2.0$ & $0.16-0.18$ & 7.9-9.0 & $<2$ & Low \\
\hline \multirow[t]{5}{*}{ Ulm } & Ap & $0-4$ & $\mathrm{C}$ & 28-35 & $1.15-1.25$ & $0.6-2.0$ & $0.17-0.21$ & 6.6-7.3 & $<2$ & Moderate \\
\hline & Bt1 & $4-18$ & & $35-50$ & $1.20-1.30$ & $0.06-0.2$ & $0.17-0.21$ & $6.6-7.8$ & $<2$ & High \\
\hline & Bt2 & $18-25$ & & $30-42$ & $1.20-1.30$ & $0.2-0.6$ & $0.17-0.21$ & 7.9-9.0 & $<2$ & High \\
\hline & Btk & $25-33$ & & $30-42$ & $1.20-1.30$ & $0.2-0.6$ & $0.17-0.21$ & 7.9-9.0 & $<2$ & High \\
\hline & $\mathrm{Ck}$ & $33-53$ & & $30-42$ & $1.20-1.30$ & $0.2-0.6$ & $0.17-0.21$ & 7.9-9.0 & $<2$ & High \\
\hline \multirow[t]{3}{*}{ Kishona } & A & $0-5$ & B & $20-27$ & $1.20-1.30$ & $0.6-2.0$ & $0.15-0.17$ & $7.4-8.4$ & $<2$ & Low \\
\hline & $\mathrm{Bw} / \mathrm{k}$ & $5-11$ & & $20-35$ & $1.20-1.35$ & $0.6-2.0$ & $0.16-0.20$ & 7.9-9.0 & $<4$ & Moderate \\
\hline & C & $11-55$ & & $20-35$ & $1.20-1.35$ & $0.6-2.0$ & $0.16-0.20$ & 7.9-9.0 & $<4$ & Moderate \\
\hline
\end{tabular}

1. Data taken from the Soil Survey of Sheridan County, Wyoming (USDA-NRCS, 1986) 


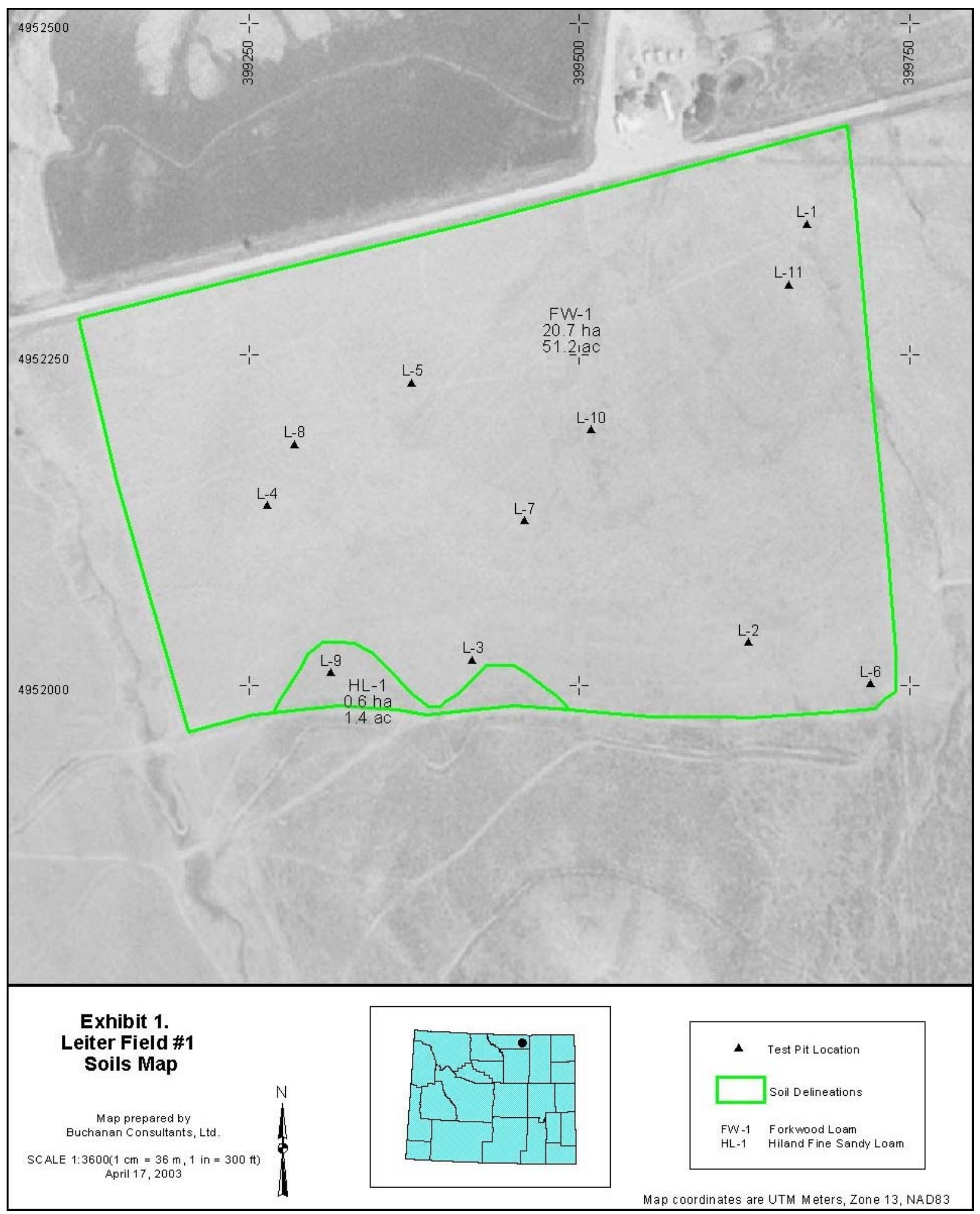




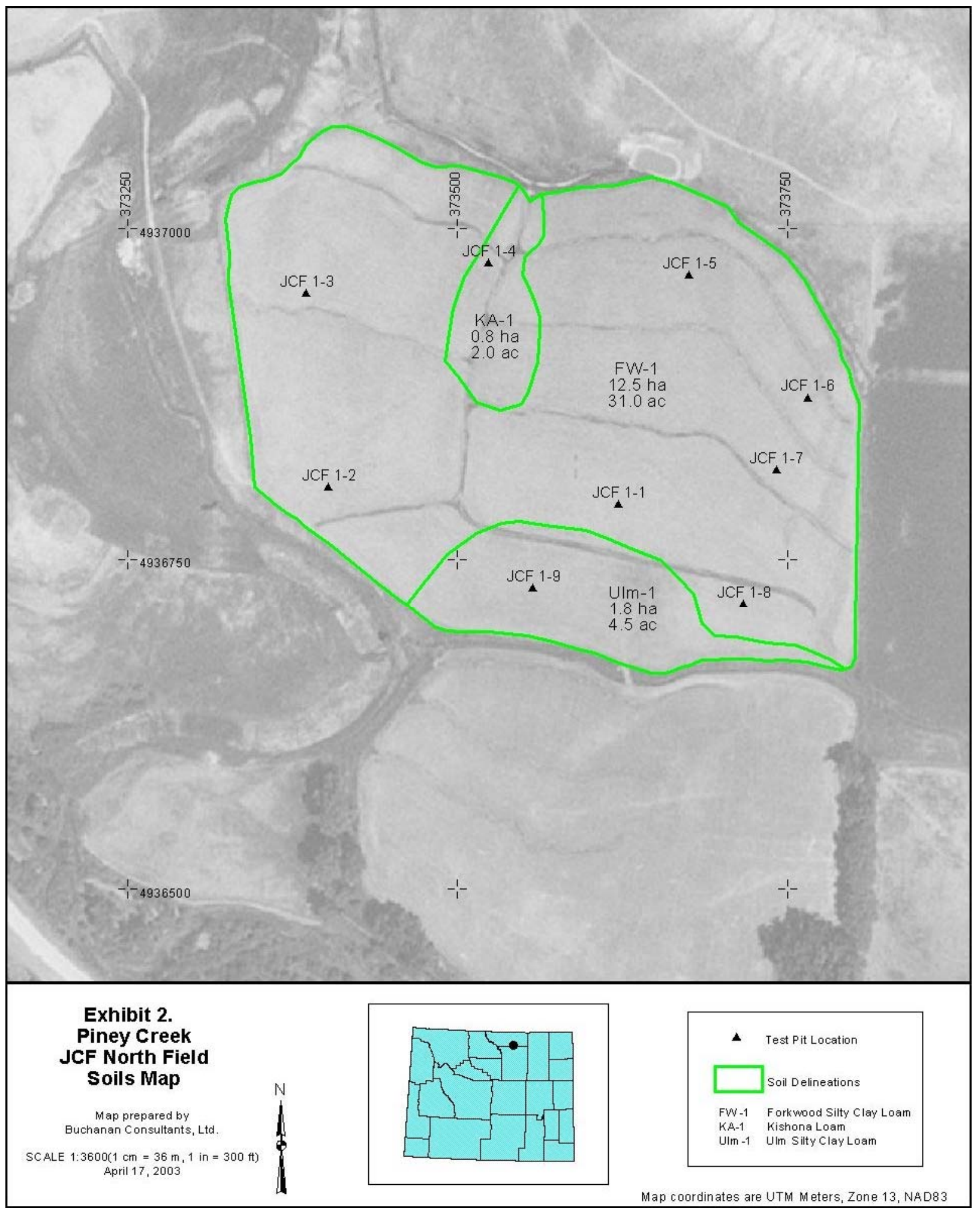


Appendix I-A

\section{Leiter Field \#1 Soil Profile Descriptions}




\section{Soil Description}

Soil Type Forkwool / ulm

File No

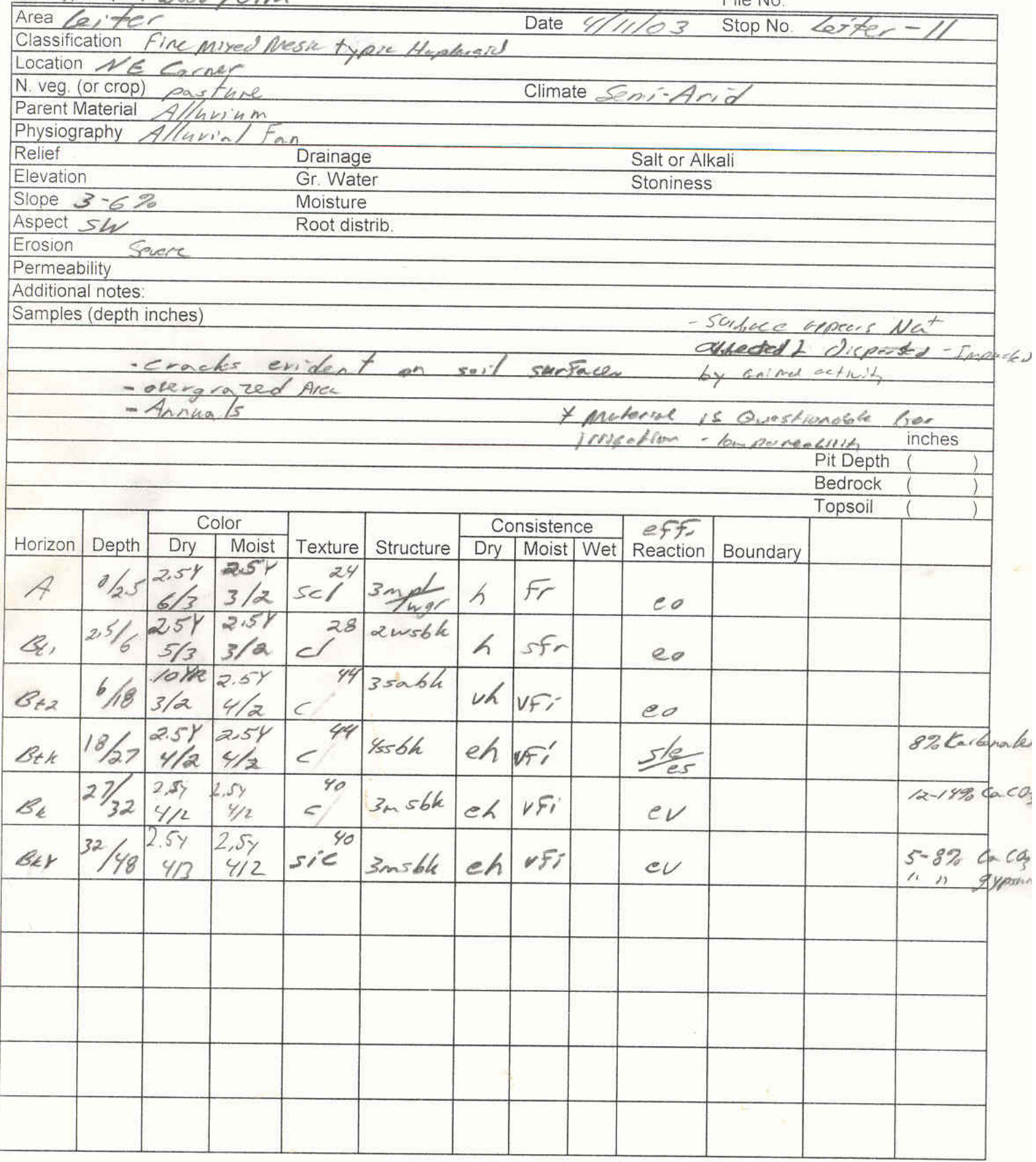




\section{Soil Description}

Soil Type

Area Le,terl File No.

Classification

Date $2 / 19 / 03$

Stop No. $\mathrm{LI}$

Location Le, ifer

N. veg. (or crop)

Parent Material

Physiography

Relief

Elevation

Slope 0-6\%

Aspect $\mathbb{N}$

Erosion -

Permeability

Additional notes:

Samples (depth inches) 3 samplew taken - o->" $>-15^{\prime \prime} 15-30 " 1$

\begin{tabular}{|c|c|c|c|c|c|c|c|c|c|c|c|c|}
\hline \multirow[b]{5}{*}{ Horizon } & \multirow[b]{5}{*}{ Depth } & \multirow{4}{*}{\multicolumn{2}{|c|}{ Color }} & \multirow[b]{5}{*}{ Texture } & \multirow[b]{5}{*}{ Structure } & & & & & & Pit Depth & $(1)$ \\
\hline & & & & & & & & & & & Bedrock & 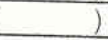 \\
\hline & & & & & & & & & & & Topsoil & ) \\
\hline & & & & & & \multicolumn{3}{|c|}{ Consistence } & \multirow{2}{*}{$\begin{array}{l}10 \% \mathrm{AC} \\
\text { Reaction }\end{array}$} & \multirow{2}{*}{$\begin{array}{l}\angle F \% \\
\text { Boundary }\end{array}$} & & \\
\hline & & \begin{tabular}{|l|} 
Dry \\
\end{tabular} & Moist & & & Dry & \begin{tabular}{|l|l} 
Moist \\
\end{tabular} & Wet & & & & \\
\hline$A_{0}$ & $0->11$ & $\begin{array}{l}2.51 \\
513\end{array}$ & $\begin{array}{l}2.54 \\
4 / 2\end{array}$ & $\begin{array}{c}32 \% \\
<< \\
\end{array}$ & 舟 & & & & es & $0 \%$ & & \\
\hline$B_{f}$ & $7 \times 5^{\circ}$ & $\begin{array}{c}2,5 / 1 \\
5 / 3\end{array}$ & $\begin{array}{l}3,54 \\
3 / 2\end{array}$ & $\begin{array}{l}33 \% \\
c< \\
\end{array}$ & & & & & eo & $0 \%$ & & \\
\hline$B_{2}$ & $15-304$ & $\begin{array}{l}2.5 \% \\
5 / 3\end{array}$ & $\begin{array}{l}254 \\
4 / 2\end{array}$ & $\begin{array}{l}32 \% \\
<< \\
\end{array}$ & & & & & eo & $0 \%$ & & \\
\hline & & & & & & & & & & & & \\
\hline & & & & & & & & & & & & \\
\hline & & & & & & & & & & & & \\
\hline & & & & & & & & & & & & \\
\hline & & & & & & & & & & & & \\
\hline & & & & & & & & & & & & \\
\hline & & & & & & & & & & & & \\
\hline & & & & & & & & & & & & \\
\hline
\end{tabular}




\section{Soil Description}

Soil Type

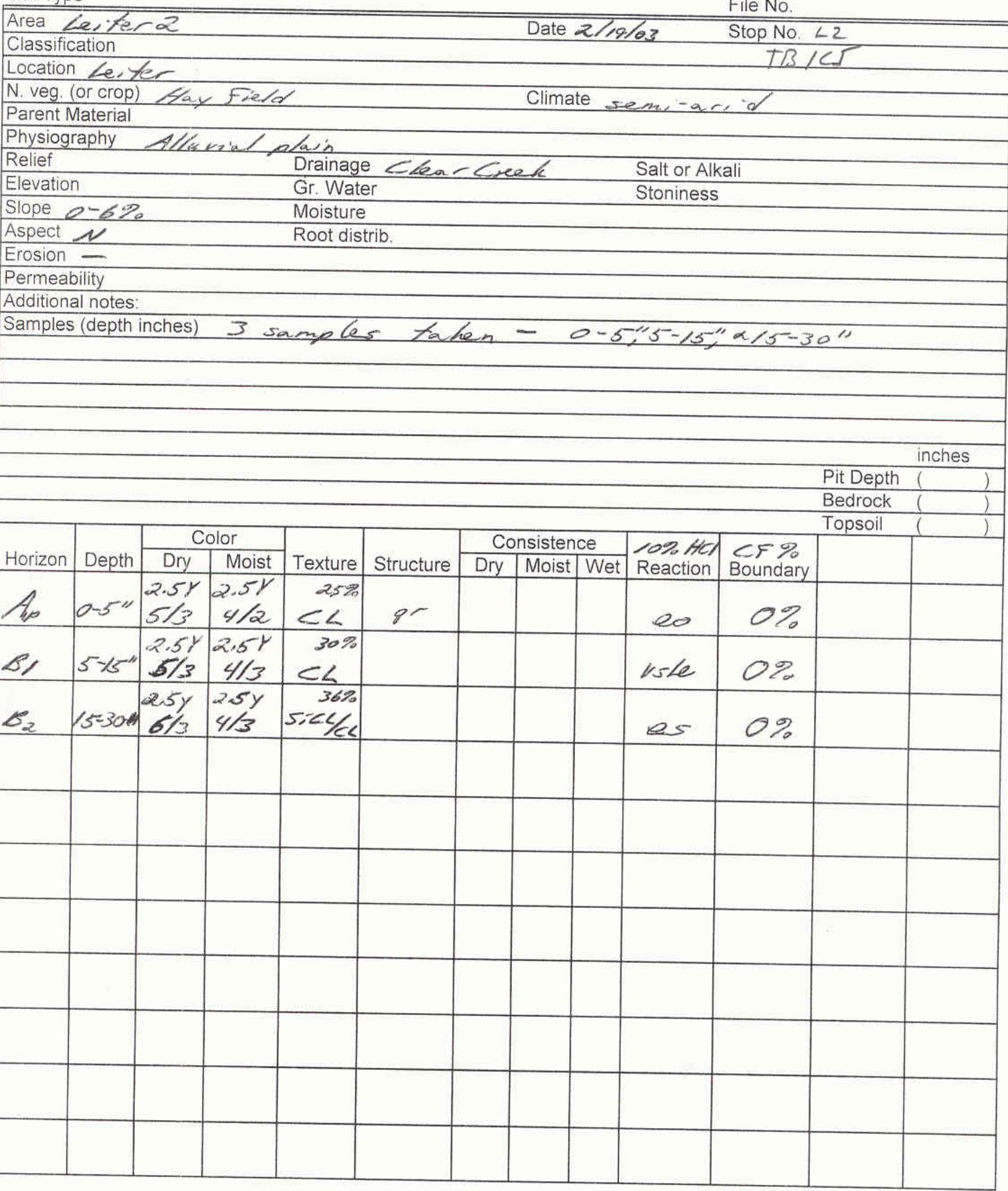




\section{Soil Description}

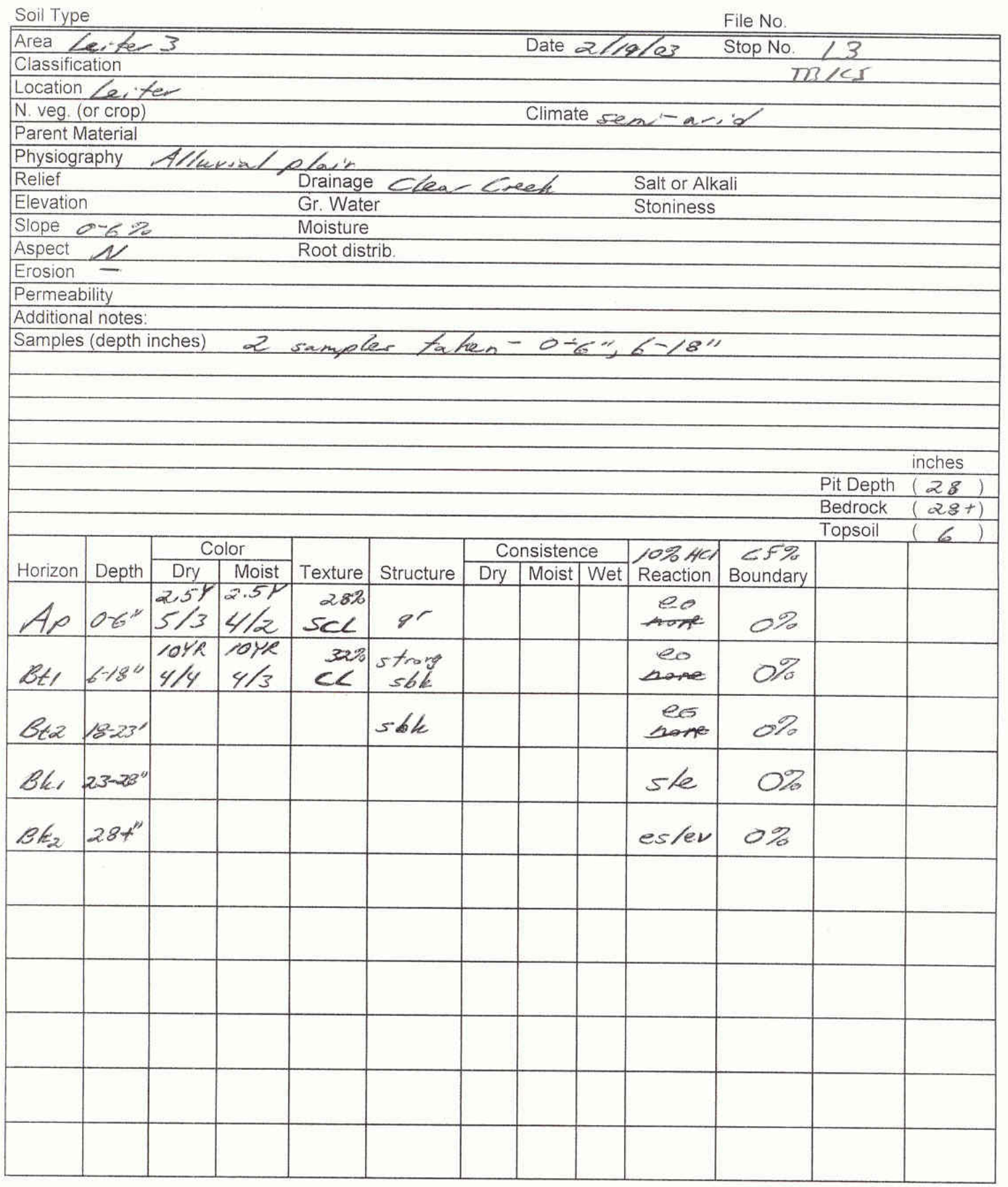


Soil Description

Soil Type

File No.

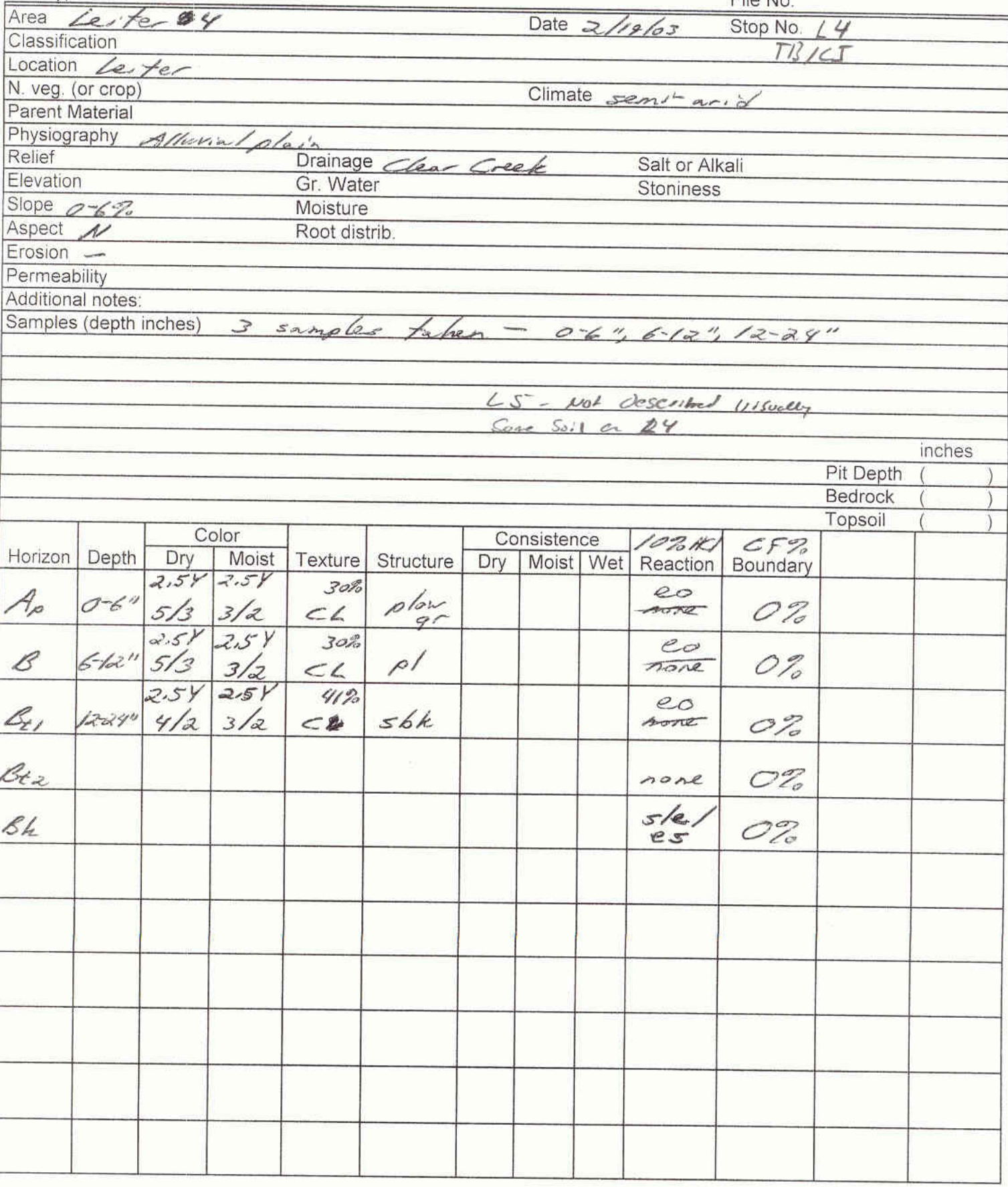




\section{Soil Description}

Soil Type Fork wool $/ 1 / \mathrm{m}$

File No

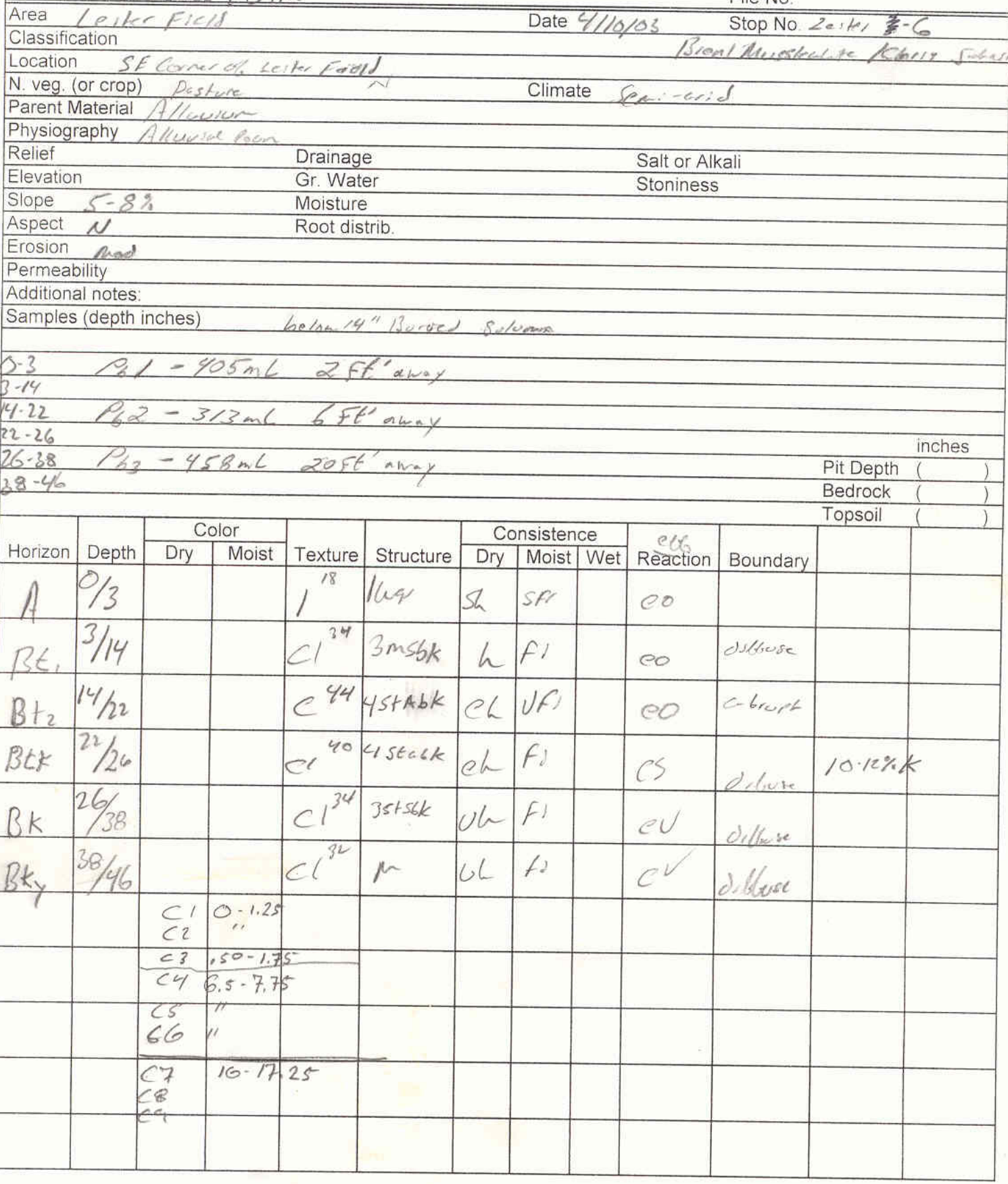




\section{Soil Description}

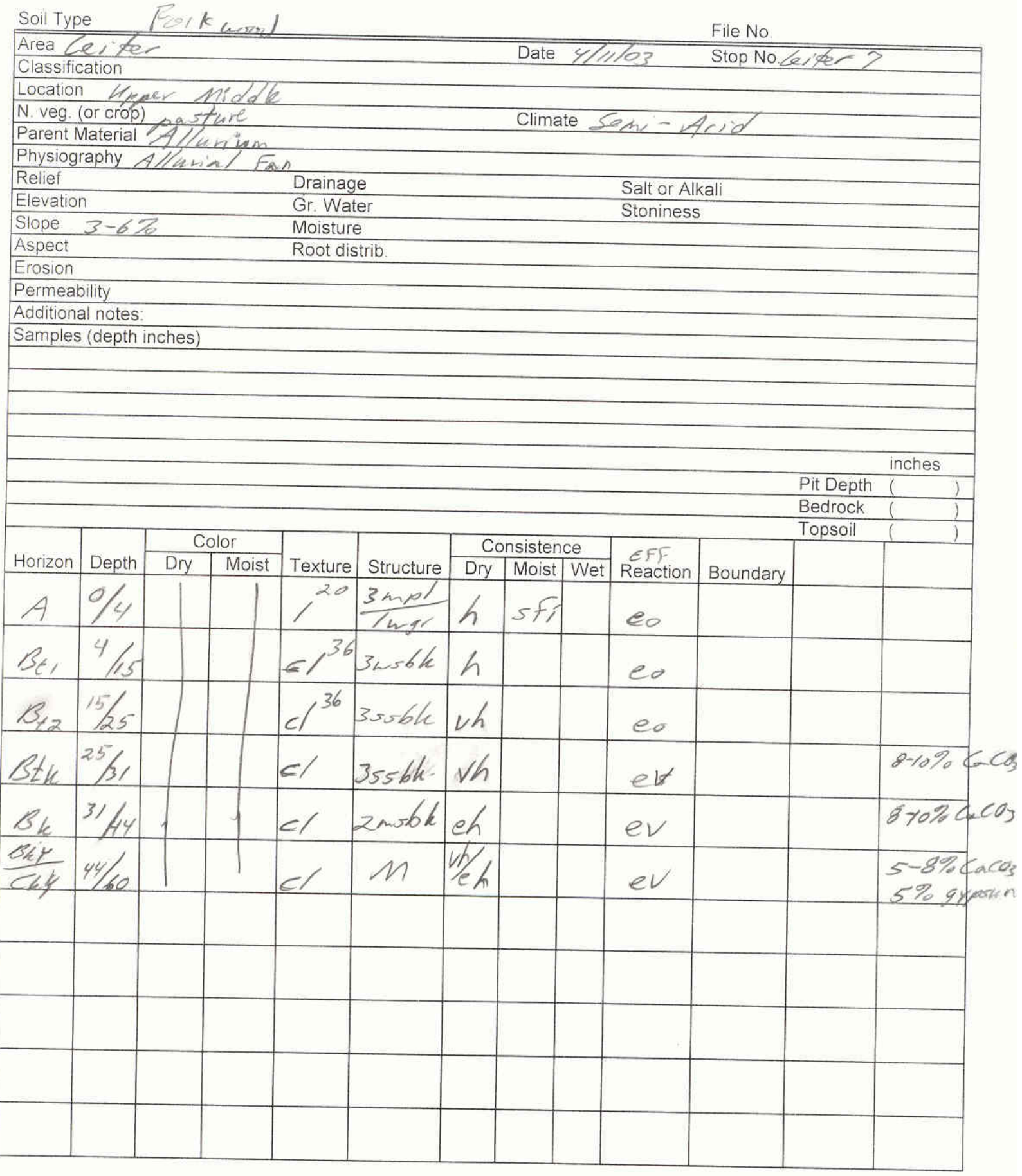




\section{Soil Description}

Soil Type Fork

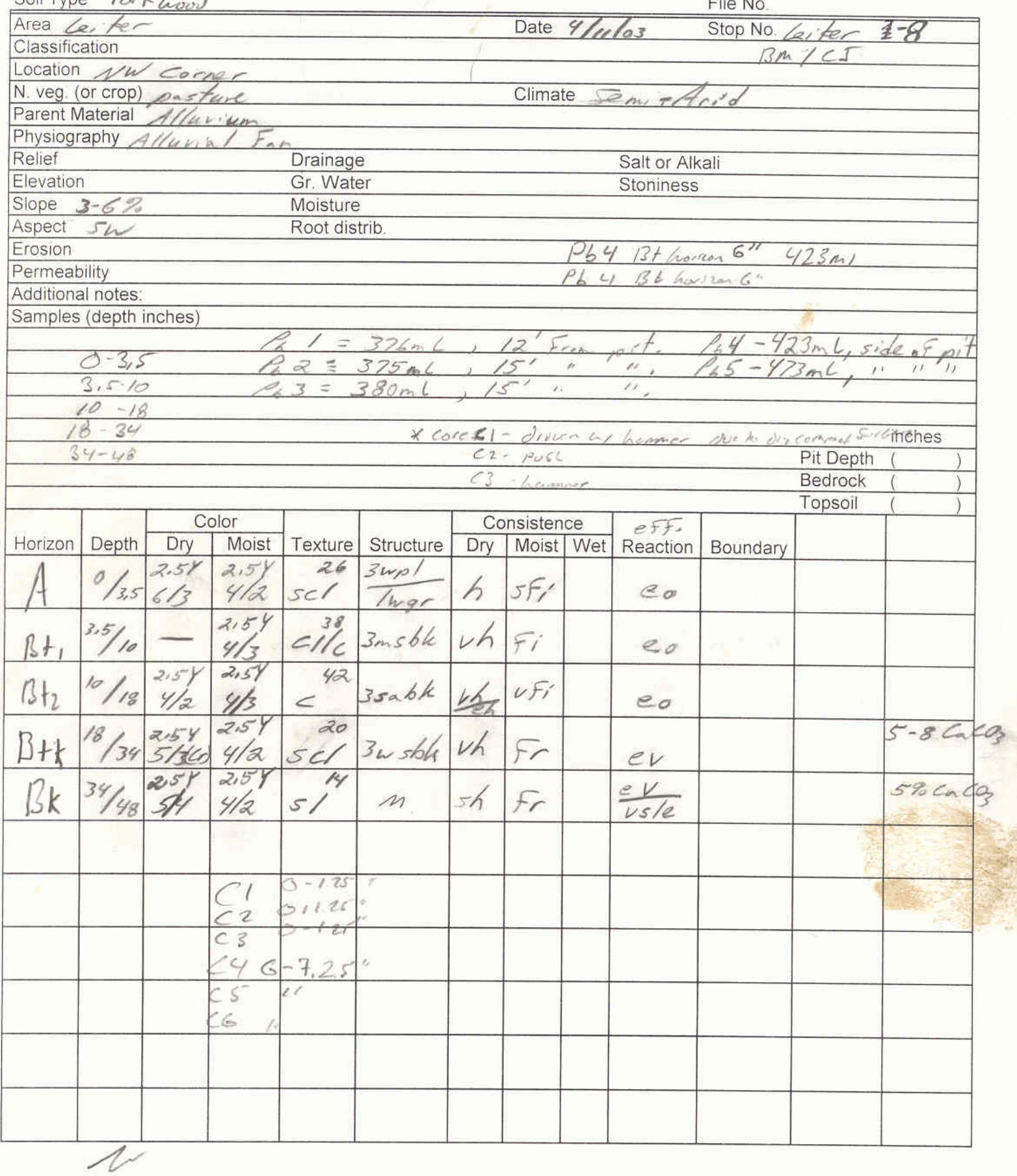


Soil Description

Soil Type H iland

File No.

Area Le, 'te -

Date 4711103 Stop No leifer 9

Classification Pine loem Mwed hesk ustre thendared

Location $5 \mathrm{~h}$ corne-

N. veg. (or crop) ansture

Climate

Parent Material eolian

Physiography mids lo,e of pa leadune

Relief

Elevation

Drainage

Slope

Aspect Root distrib

Erosion

Permeability

Additional notes:

Samples (depth inches)

\begin{tabular}{|c|c|c|c|c|c|c|c|c|c|c|c|c|}
\hline \multirow[b]{5}{*}{ Horizon } & \multirow[b]{5}{*}{ Depth } & \multirow{4}{*}{\multicolumn{2}{|c|}{ Color }} & \multirow[b]{5}{*}{ Texture } & \multirow[b]{5}{*}{ Structure } & \multirow{4}{*}{\multicolumn{3}{|c|}{ Consistence }} & \multirow{5}{*}{$\begin{array}{c}E F F \\
\text { Reaction }\end{array}$} & \multirow{3}{*}{\multicolumn{2}{|c|}{$\begin{array}{l}\text { Pit Depth } \\
\text { Bedrock } \\
\text { Topsoil }\end{array}$}} & inches \\
\hline & & & & & & & & & & & & ) \\
\hline & & & & & & & & & & & & ) \\
\hline & & & & & & & & & & & & \\
\hline & & \begin{tabular}{|l|} 
Dry \\
\end{tabular} & Moist & & & Dry & Moist & Wet & & Boundary & & \\
\hline$A$ & $1 / 2$ & $\begin{array}{l}2,5 / \\
5 / 3\end{array}$ & $\begin{array}{l}101 k \\
4 / 3\end{array}$ & $\mathrm{Fs} /^{14}$ & 3npl & $5 h$ & fr & & eo & & & \\
\hline Bt, & 2 & $\begin{array}{l}\text { lo1k } \\
4 / 4\end{array}$ & $\begin{array}{l}10 \mathrm{KR} \\
4 / 3\end{array}$ & $51^{18}$ & $2 w-6 k$ & sh & Fr & & eo & & & \\
\hline$B_{t_{2}}$ & 8 & $\begin{array}{l}10122 \\
414\end{array}$ & $\begin{array}{l}10 Y R \\
4 / 3\end{array}$ & $5 \mathrm{cl}$ & 45 bh & vh & $5 F r$ & & eo & & & \\
\hline$B_{3}$ & & $\begin{array}{l}10 Y 2 \\
4 / 3\end{array}$ & $\begin{array}{l}101 R \\
4 / 3\end{array}$ & $5=l^{22}$ & Yms sbhe & wh & Fr & & eo & & & \\
\hline$B_{k}$ & $35 / 48$ & $\begin{array}{l}2.54 \\
612 \\
\end{array}$ & $\begin{array}{l}10 K \\
4 / 2\end{array}$ & Lf5/ & $\frac{3 w 56 k}{M}$ & $\frac{h}{5 h}$ & & & ev & & & $\begin{array}{l}15-189 \\
\text { cale1 }\end{array}$ \\
\hline & & & & & & & & & & & & \\
\hline & & & & & & & & & & & & \\
\hline & & & & & & & & & & & & \\
\hline & & & & & & & & & & & & \\
\hline & & & & & & & & & & & & \\
\hline & & & & & & & & & & & & \\
\hline
\end{tabular}




\section{Soil Description}

Soil Type Fork waod $/ U / \mathrm{M}$

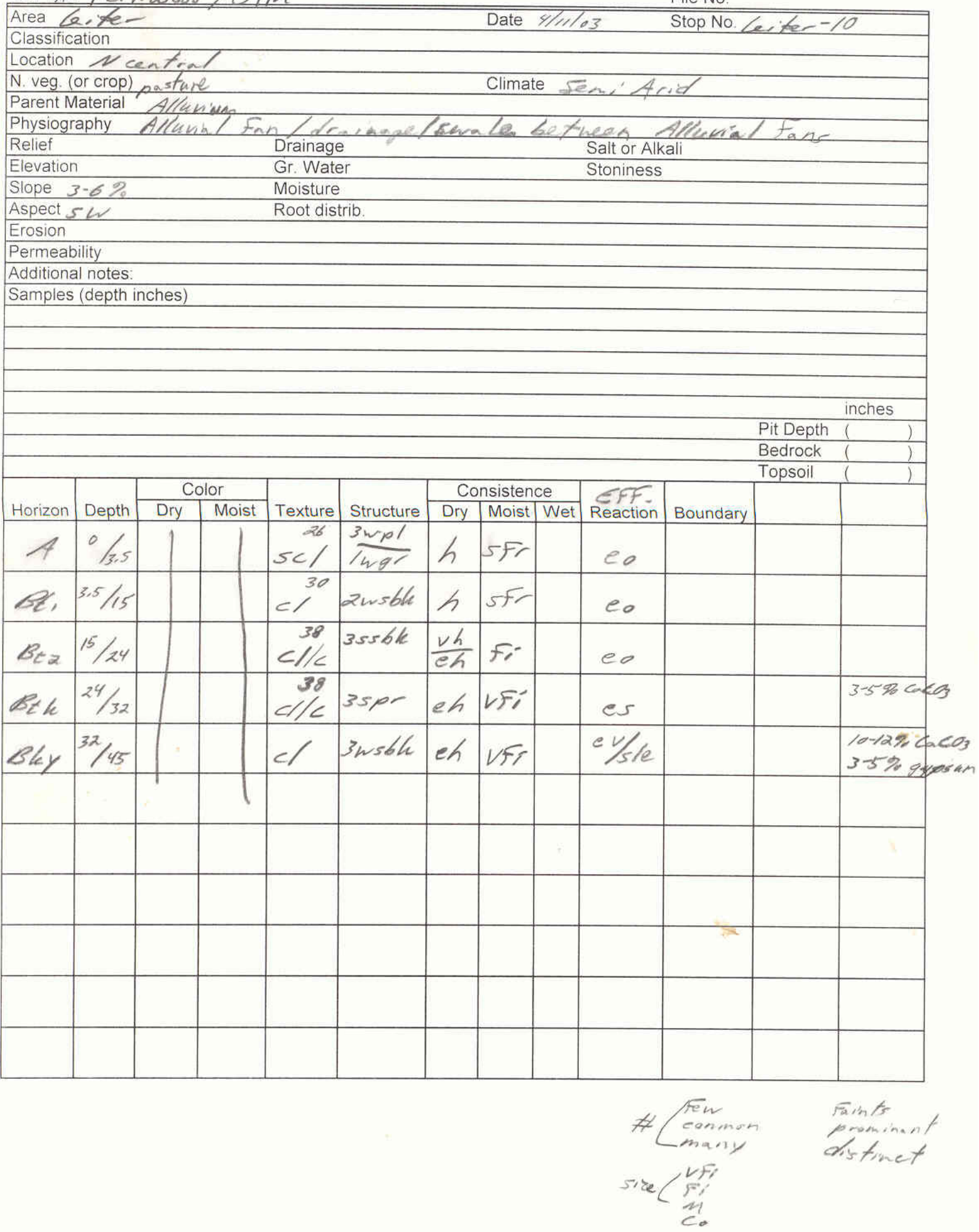


Appendix I-B

Cooksley Ranch Study Site Soil Profile Descriptions 


\section{Soil Description}

Soil Type $\mathrm{C} / \mathrm{m}$

File No.

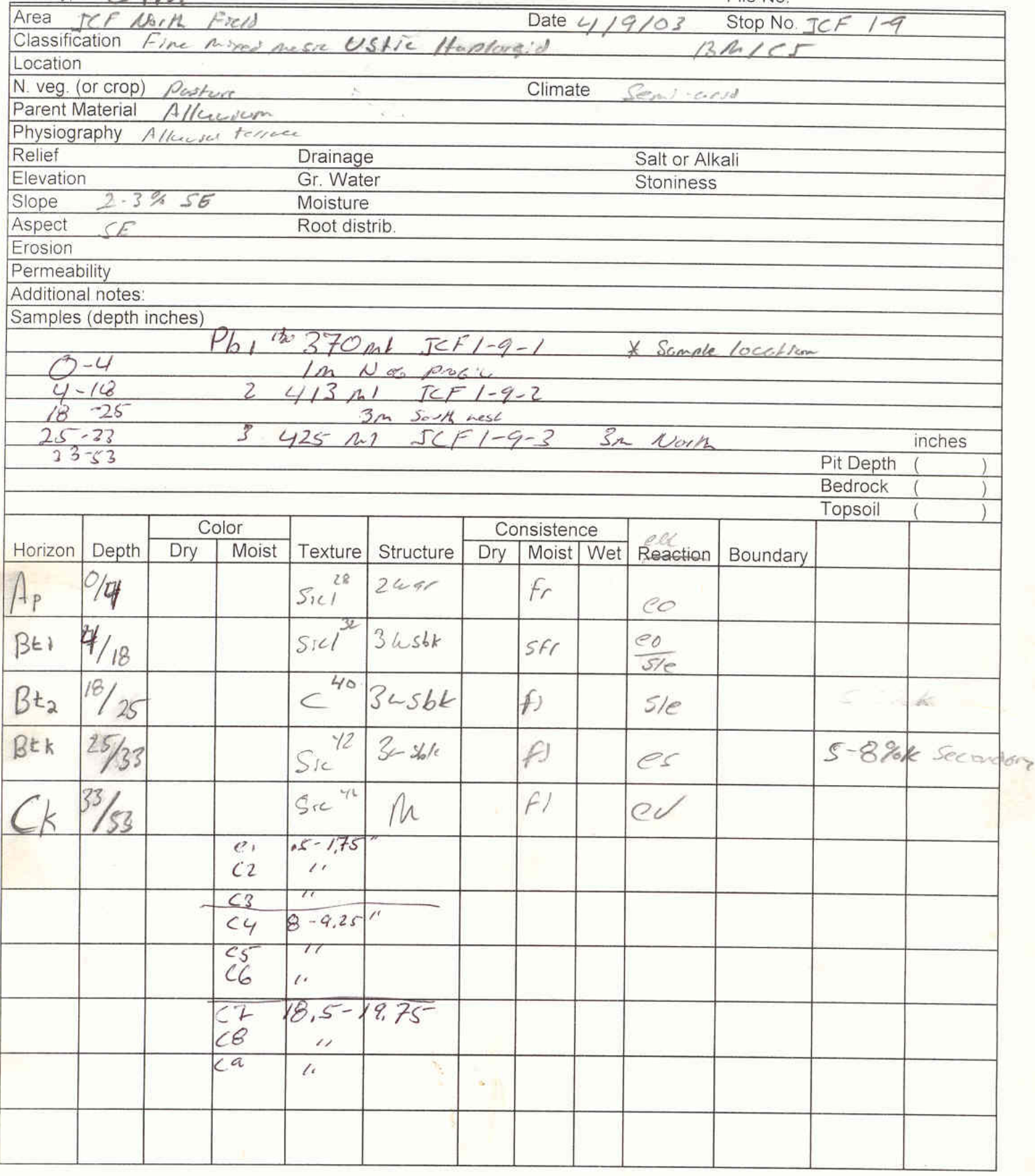




\section{Soil Description}

Soil Type Forklewor

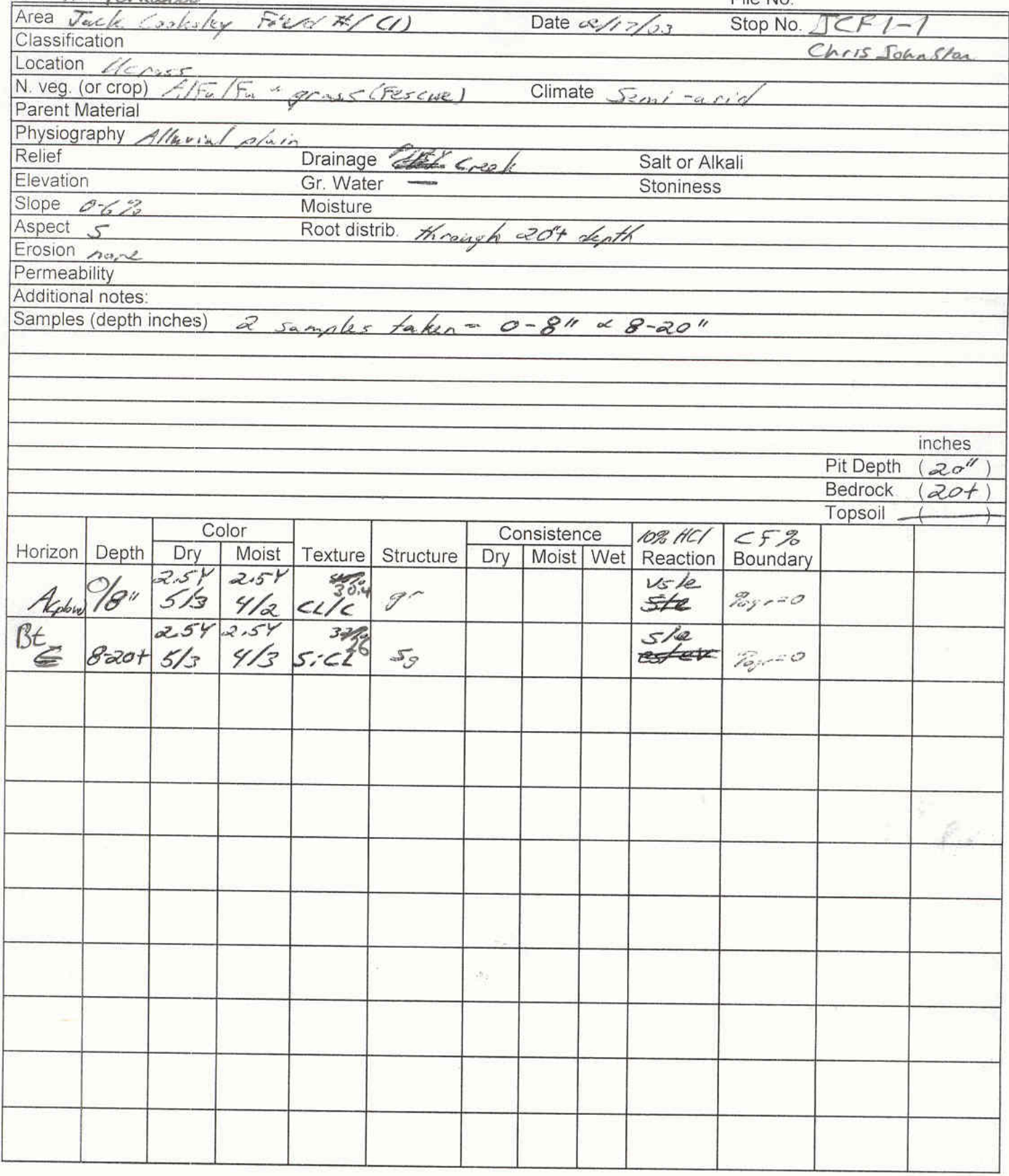

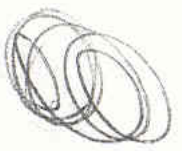




\section{Soil Description}

Soil Type Fork wodd

Area Tack Cooksbys $\quad$ File No

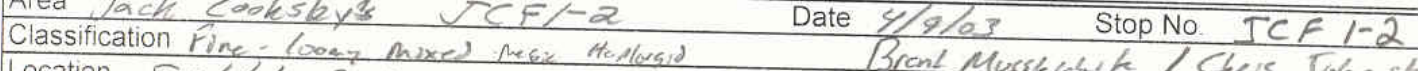

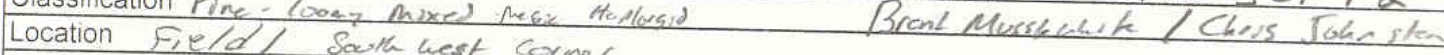

N. veg. (or crop)

Parent Material Alluvium Climate Semi-Arid

Physiography Alluvid Eerrace

Relief

Elevation 4/00

Drainage

Salt or Alkali

Slope $1-30$ Gr. Water

Aspect sacth

Moisture

Stoniness

Erosion Sligel

Permeability

Additional notes:

Samples (depth inches)

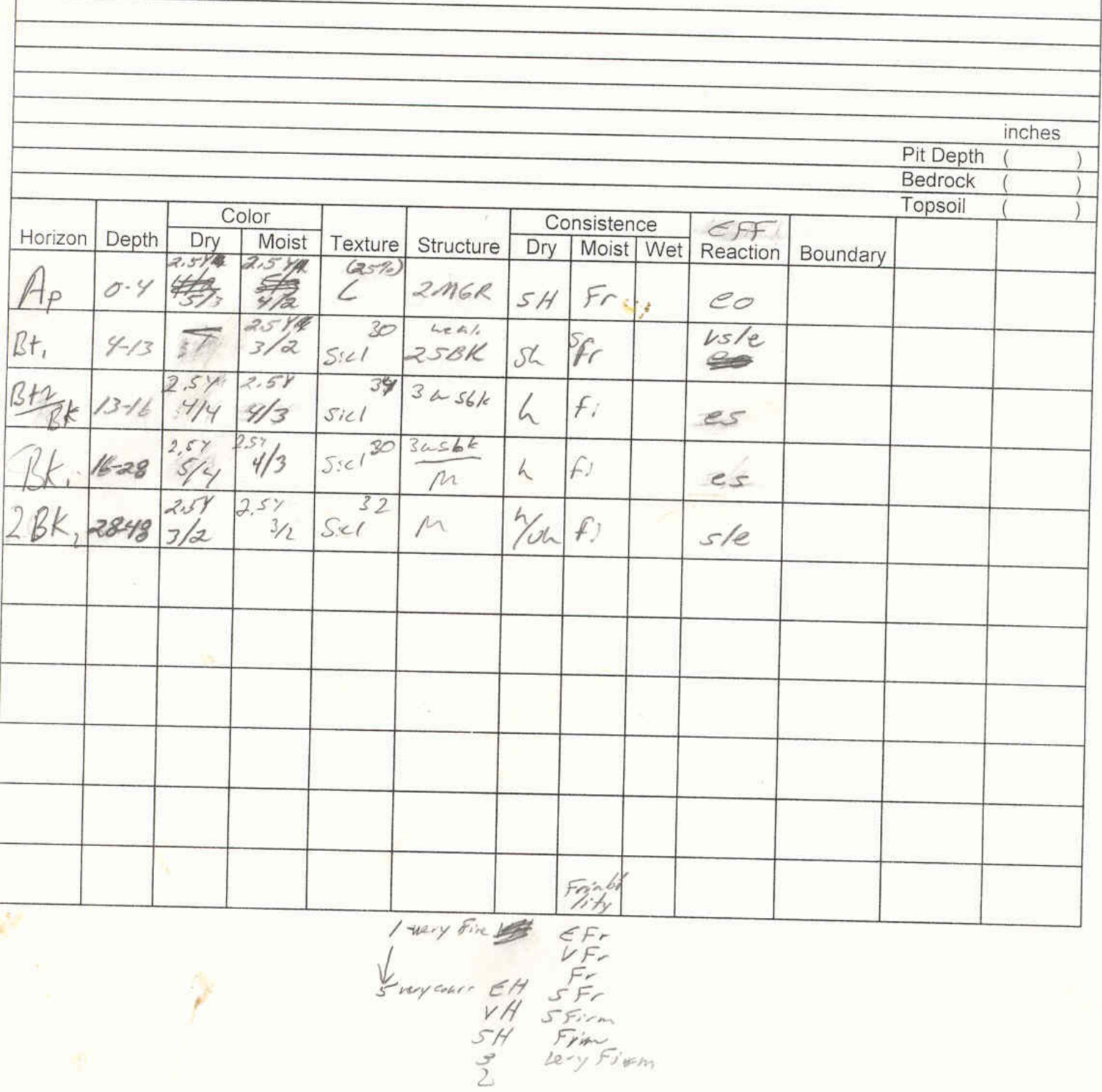




\section{Soil Description}

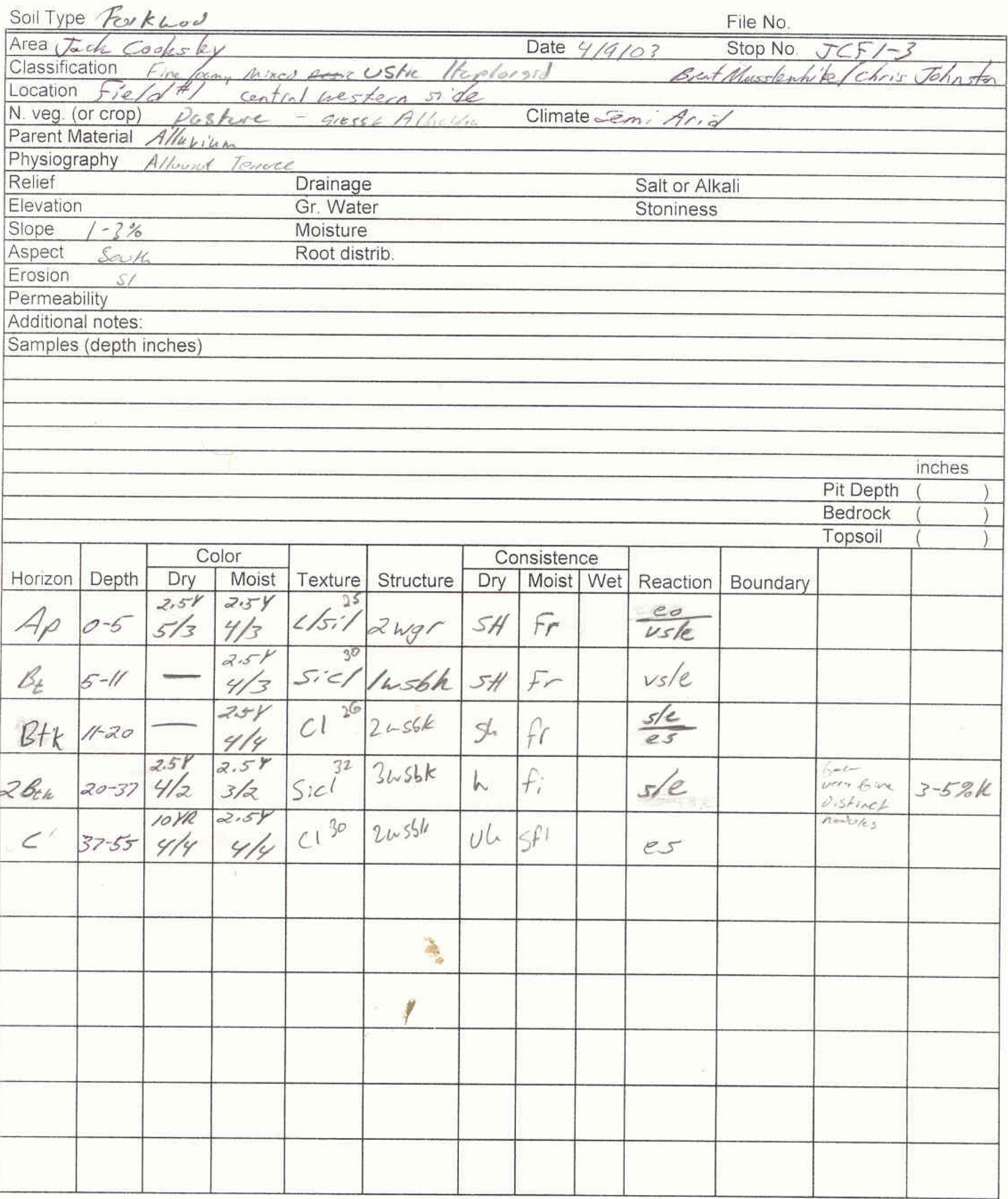




\section{Soil Description}

Soil Type 'Kishona'-loam File No

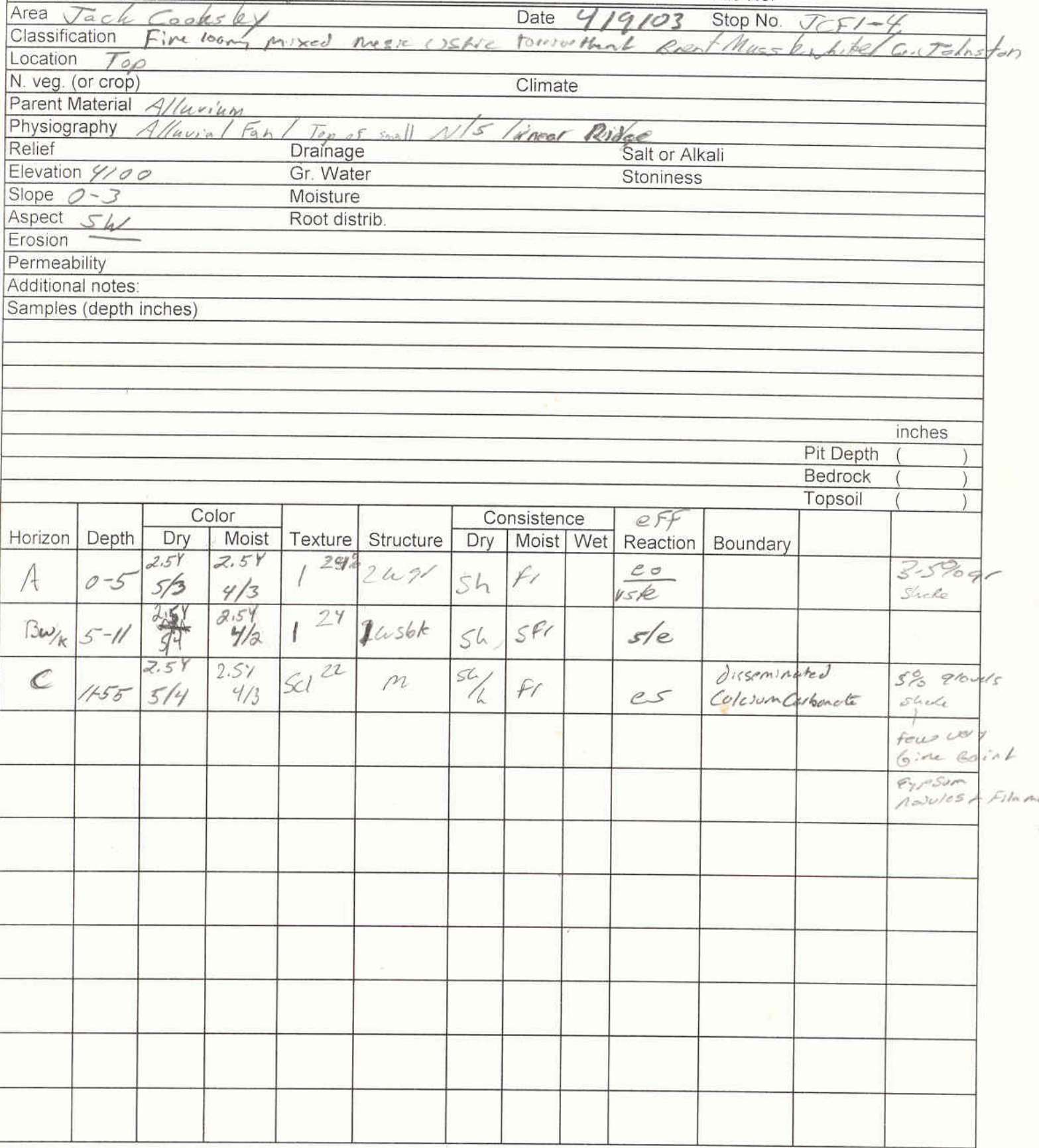




\section{Soil Description}

Soil Type Forkuand

File No.

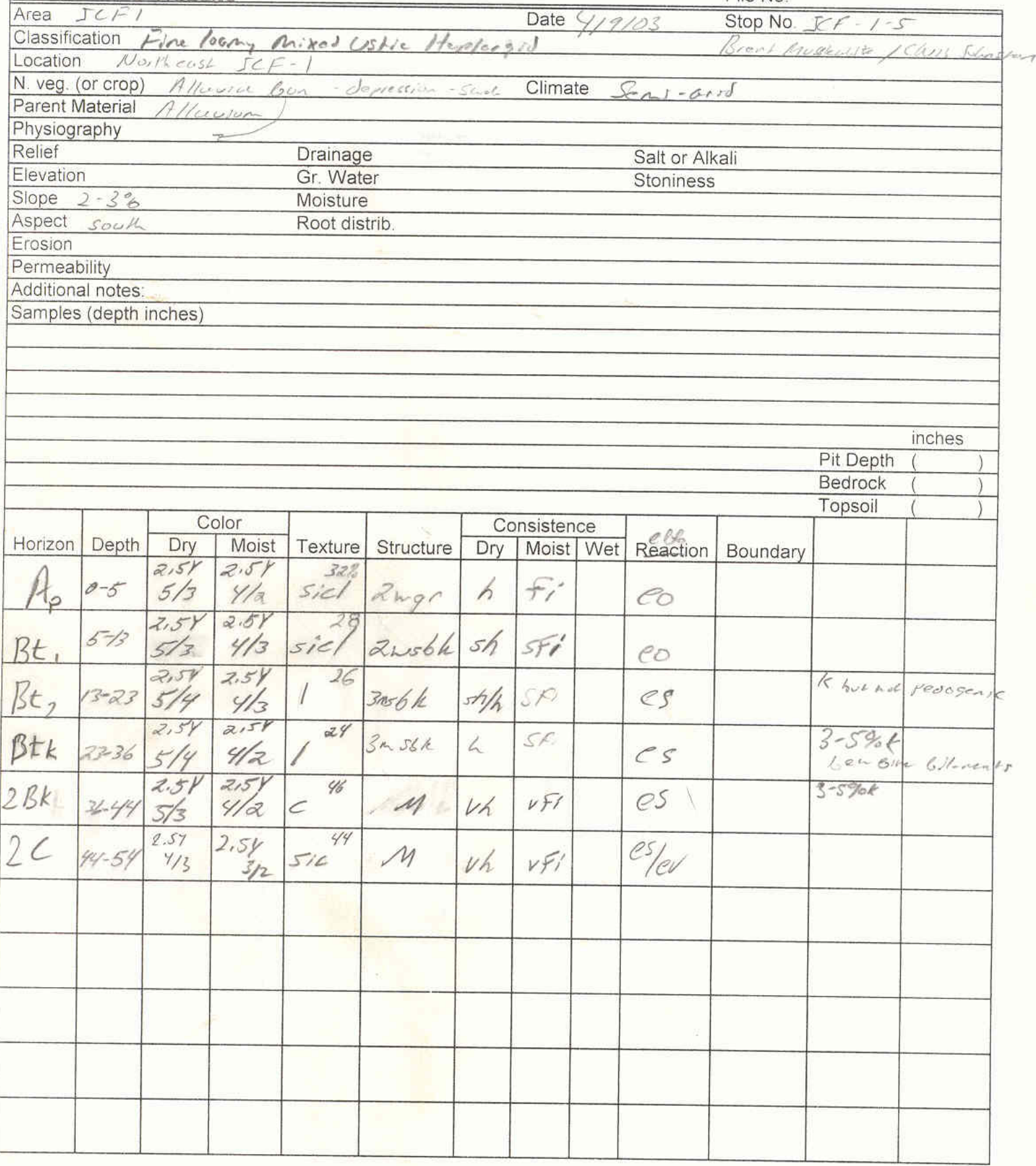




\section{Soil Description}

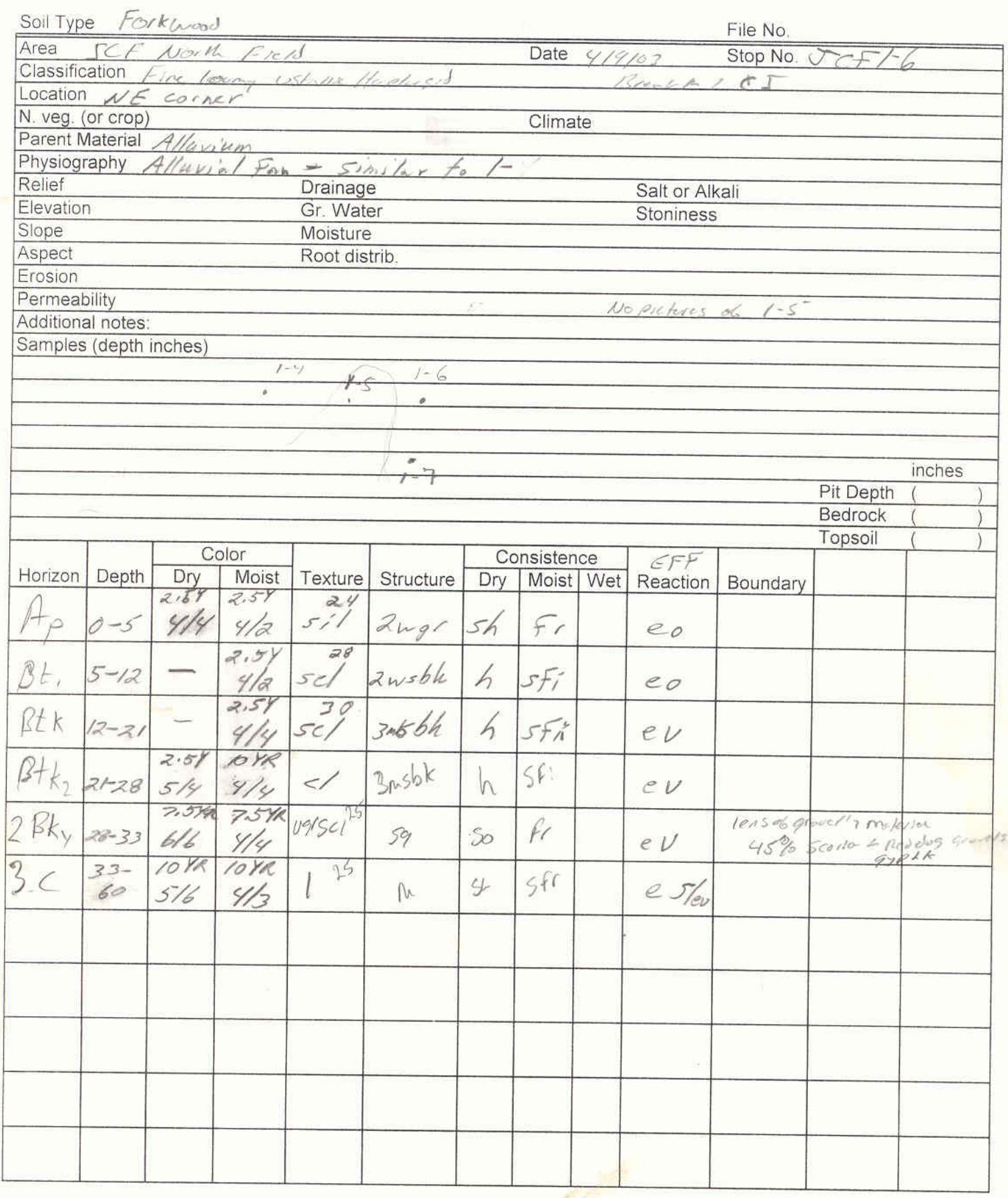




\section{Soil Description}

Soil Type Forkwod

\begin{tabular}{|c|c|c|c|c|c|c|c|c|c|c|c|c|}
\hline & & & & & & & & & & File No. & & \\
\hline Area & ICF & & Pacy) & & & & Date & $4 /$ & $4 / 08$ & Stop No. & $\Gamma \subset F=1$ & $1-7$ \\
\hline Classific & ation & & & & & & & & & $\mathrm{B} / \mathrm{L}$ & $\angle 5$ & \\
\hline Location & $E_{c}$ & ist $\mathrm{Ce}$, & atrel 1 & coted of / & wath Feld & & & & & & & \\
\hline N. veg. ( & or crop) & Past & fure & & & & Climat & & Sem:- & $\ldots, 1$ & & \\
\hline Parent M & laterial & Allu & - wern & $-x+2$ & & & & & & & & \\
\hline Physiogr & aphy & Esin & & & & & & & & & & \\
\hline Relief & & & & Drainage & & & & & Salt or Alk & kali & & \\
\hline Elevation & & & & Gr. Wate & & & & & Stoniness & & & \\
\hline Slope & $3 \%$ & & & Moisture & & & & & & & & \\
\hline Aspect & Soe & & & Root distr & trib. & & & & & & & \\
\hline Erosion & & & & & & & & & & & & \\
\hline Permeab & Dility & & & & & & & & & & & \\
\hline Additiona & al notes: & & & & & & & & & & & \\
\hline Samples & (depth if & ches) & & & imsath & & & & ime pou & Gra ar & $I C I^{E} /-C$ & \\
\hline & & & $P b$ & $\therefore 285$ & $5 \mathrm{ml} \mathrm{HzO}$ & $1-7$ & & & & & & \\
\hline $0-4$ & & & $\mathrm{~Pb}=$ & $=205$ & $5 \mathrm{ml} \mathrm{H} \mathrm{H}_{2} \mathrm{O}$ & $1.7-$ & & $*$ & Potentreal & Semple loce & ection & \\
\hline $4-11$ & & & & & Ecost & & & & & & & \\
\hline 11.23 & & & & 500 & mi 1 & $1-7-$ & & & & 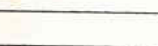 & 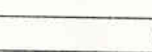 & inches \\
\hline $23 \cdot 30$ & & & & 2 & m west? & & & & & $x^{2}$ & Pit Depth & ( \\
\hline $30-4 / 2$ & & & & & & & & & & 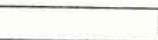 & Bedrock & 1 \\
\hline$y^{2}-50$ & & & & & & & & & & & Topsoil & \\
\hline & & & & & & & onsisten & nce & & & & \\
\hline Horizon & Depth & \begin{tabular}{l|}
\multicolumn{2}{r}{ Co } \\
Dry
\end{tabular} & $\begin{array}{l}\text { olor } \\
\text { Moist }\end{array}$ & Texture & Structure & \begin{tabular}{|l|} 
Co \\
Dry
\end{tabular} & Moist & Wet & Reaction & Boundary & & \\
\hline$A_{p}$ & $0 / 4$ & & & $5.1^{26}$ & 2 mar & SL & Fr & & eo & & & \\
\hline Bt, & $4 / 11$ & & & Siel ${ }^{32}$ & 2usble & h & fi & & eo & & & \\
\hline$B t_{2}$ & $11 / 23$ & & & $\mathrm{Sed}^{34}$ & 3 msble & h & A) & & eo & & & \\
\hline BtK & $23 / 30$ & & & $\mathrm{SiCl}^{32}$ & $3 m \leqslant b / c$ & $S$ & SFi & & ev & & $5.10 \%$ & \\
\hline $2 c k$ & $30 / 42$ & & & essel & 59 & So & & & ev & & G50\% Sep. & 5 \\
\hline $3 c k$ & $42 / 50$ & & & 122 & $\mu$ & Sh & & & es & & & \\
\hline & & $50: 1$ & Ring 17 & 1 . 5 to & $1.75^{\prime \prime}$ & & & & & & & \\
\hline & & So:11 & $\begin{array}{c}R^{2} \\
c^{3}\end{array}$ & $\begin{array}{c}k=-8 \\
y\end{array}$ & $7-81 / 4$ & " & & & & & & \\
\hline & & & $\angle 4$ & " & & & & & & & & \\
\hline & & & $c 5$ & .5 to & $1.75^{\prime \prime}$ & & & & & & & \\
\hline & & & & & & & & & & & & \\
\hline
\end{tabular}




\section{Soil Description}

Soil Type forkwodd parderine on Comberia

Area Tack Colstors

File No.

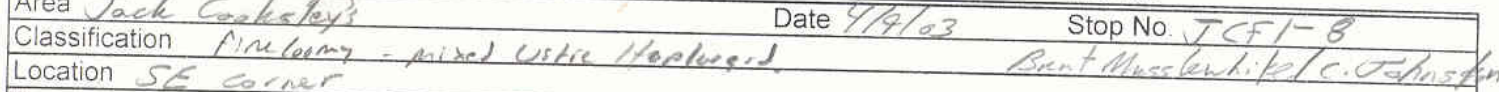

\begin{tabular}{l} 
Location $5 E$ corner \\
\hline N. veg. (or crop) Pasture
\end{tabular}

Parent Material Allukium

Physiography Alluvio/ Prrace

Relief

Elevation 4/00 Drainage

Slope Gr. Water

\begin{tabular}{ll} 
Aspect $5 \mathrm{~W}$ & Moisture \\
\hline
\end{tabular}

Erosion

Permeability

Additional notes:

Samples (depth inches)

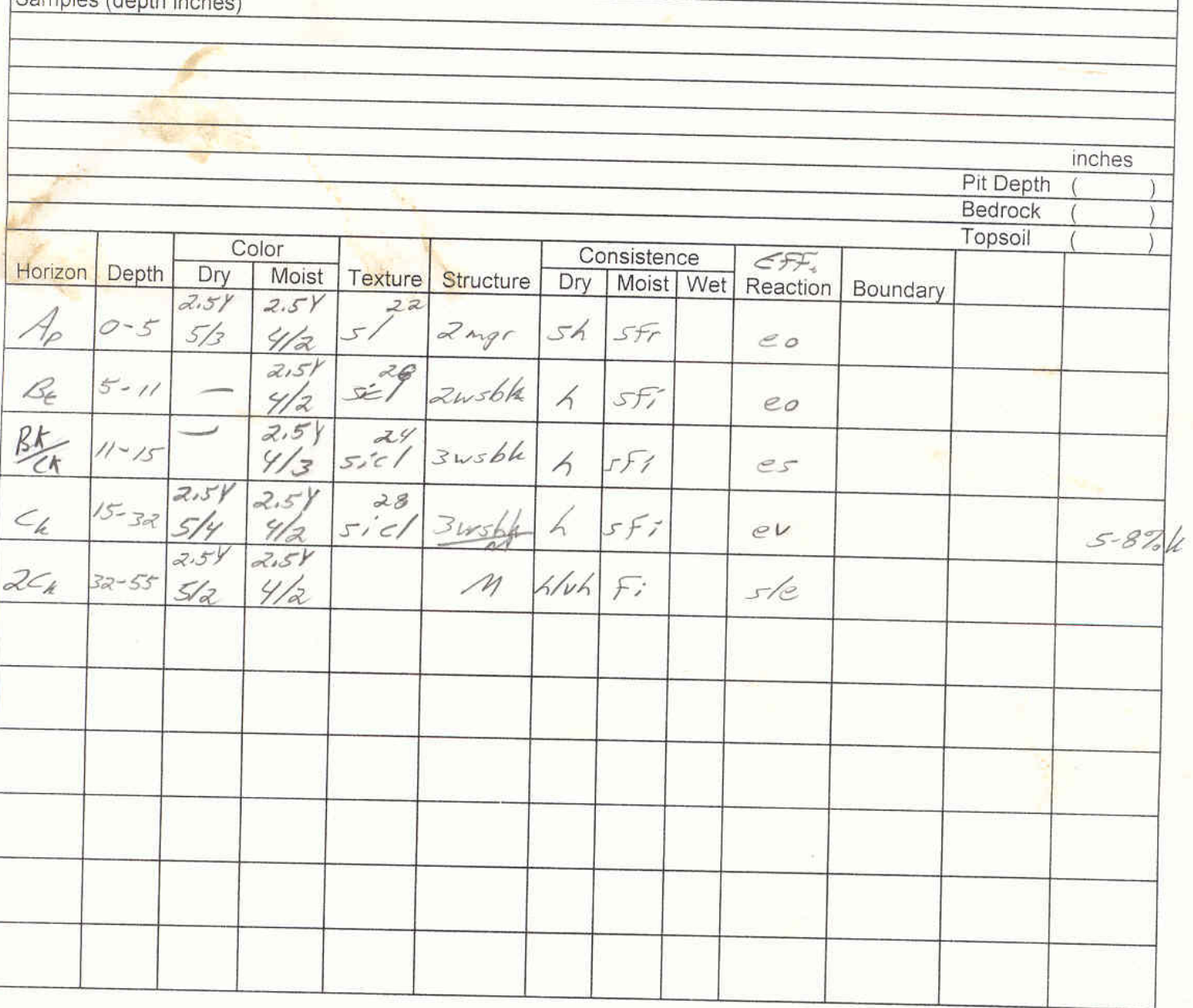




\section{Appendix II}

Baseline Water Quality Data for Clear Creek, Piney Creek and Fowler 11-18 - CBM water that will be applied to the research plots at Cooksley Ranch Research Site. 


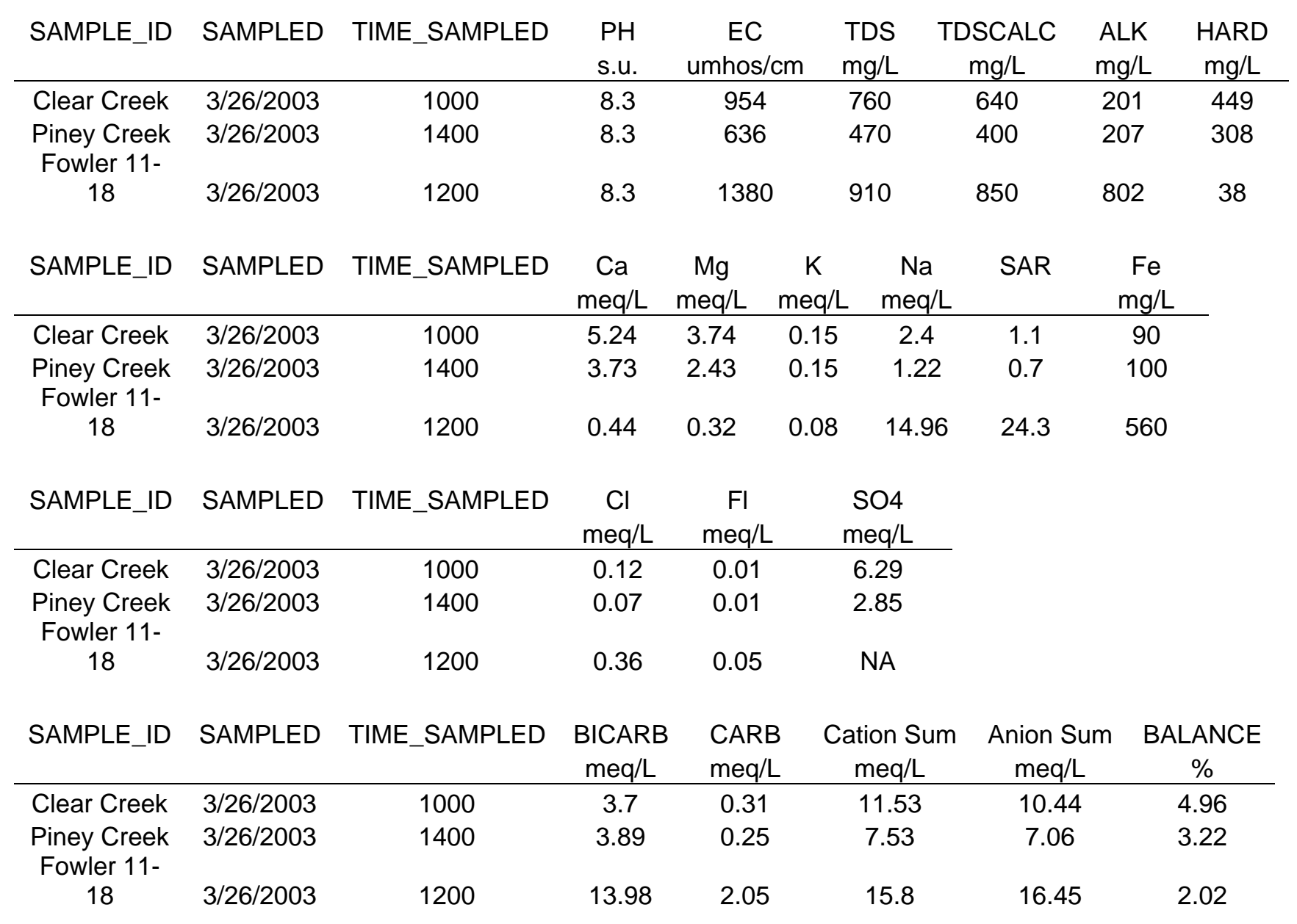




\section{Appendix III}

Chemical analysis of extracts collected from the materials used in the column studies after the treatment application. 


\begin{tabular}{|c|c|c|c|c|c|c|c|c|c|c|c|c|c|c|c|c|c|c|}
\hline & & & & $\mathrm{pH}$ & EC & Sat & $\mathrm{Mg}$ & $\mathrm{Na}$ & $\mathrm{K}$ & $\mathrm{Ca}$ & SAR & $\begin{array}{l}\text { alkalinity } \\
\mathrm{mg} / \mathrm{L} \text { as }\end{array}$ & $\mathrm{F}$ & $\mathrm{Cl}$ & PO4 & NO3 & SO4 & K \\
\hline ID & $\begin{array}{c}\text { Sample } \\
\text { Leiter A }\end{array}$ & Water & Treatment & s.u. & $\mathrm{dS} / \mathrm{m}$ & $\%$ & ppm & ppm & ppm & ppm & & CaCO3 & ppm & ppm & ppm & ppm & ppm & $\mathrm{cm} / \mathrm{s}$ \\
\hline CC-1 & $\begin{array}{l}\text { horizon } 2 / 8 \text { mix } \\
\text { Leiter A }\end{array}$ & Clear Creek & - & 8.38 & 1.21 & & 58 & 110 & 29 & 144 & 2 & 7 & 0 & 7 & 14 & 20 & 322 & $1.87 \mathrm{E}-03$ \\
\hline CC-3 & $\begin{array}{l}\text { horizon } 2 / 8 \text { mix } \\
\text { Leiter A }\end{array}$ & Clear Creek & - & 8.48 & 1.21 & & 57 & 66 & 10 & 141 & 1 & 7 & 0 & 9 & 18 & 27 & 328 & $1.88 \mathrm{E}-03$ \\
\hline CC-6 & $\begin{array}{l}\text { horizon } 2 / 8 \text { mix } \\
\text { Leiter A }\end{array}$ & Clear Creek & - & 8.47 & 1.27 & & 57 & 78 & 10 & 139 & 1 & 8 & 0 & 6 & 12 & 18 & 338 & $1.74 \mathrm{E}-03$ \\
\hline CC-7 & horizon 2/8 mix & Clear Creek & - & 8.45 & 1.21 & & 56 & 64 & 10 & 134 & 1 & 7 & 0 & 7 & 13 & 20 & 325 & $1.85 \mathrm{E}-03$ \\
\hline CBM-4 & $\begin{array}{l}\text { Leiter A } \\
\text { horizon } 2 / 8 \text { mix } \\
\text { Leiter A }\end{array}$ & Fowler 11-18 & - & 8.97 & 1.47 & & 10 & 331 & 7 & 25 & 14 & 15 & 0 & 33 & 66 & 99 & 11 & 7.53E-05 \\
\hline CBM-5 & $\begin{array}{l}\text { horizon 2/8 mix } \\
\text { Leiter A }\end{array}$ & Fowler 11-18 & - & 9.12 & 1.48 & & 11 & 353 & 8 & 26 & 15 & 15 & 0 & 31 & 63 & 94 & 9 & 6.65E-05 \\
\hline CBM-8 & horizon 2/8 mix & Fowler 11-18 & - & 8.34 & 1.39 & & 9 & 308 & 5 & 17 & 15 & 14 & 0 & 33 & 66 & 99 & 10 & 6.86E-05 \\
\hline CВM-9 & $\begin{array}{c}\text { Leiter A } \\
\text { horizon } 2 / 8 \text { mix }\end{array}$ & Fowler 11-18 & $\begin{array}{l}2.5 \mathrm{t} / \mathrm{ac} \\
\text { surface } \\
\text { gyp } \\
2.5 \mathrm{t} / \mathrm{ac} \\
\text { surface }\end{array}$ & 8.76 & 1.61 & & 11 & 375 & 7 & 33 & 14 & 17 & 0 & 22 & 43 & 64 & 18 & $7.20 \mathrm{E}-04$ \\
\hline CBM-10 & $\begin{array}{l}\text { horizon 2/8 mix } \\
\text { Leiter A }\end{array}$ & Fowler 11-18 & $\begin{array}{l}\text { gyp } \\
2.5 \text { t/ac } \\
\text { surface }\end{array}$ & 8.99 & 1.68 & & 8 & 405 & 5 & 20 & 19 & 18 & 0 & 19 & 38 & 57 & 4 & 6.89E-04 \\
\hline CBM-11 & $\begin{array}{c}\text { horizon 2/8 mix } \\
\text { Leiter A }\end{array}$ & Fowler 11-18 & $\begin{array}{l}\text { gyp } \\
2.5 \text { t/ac } \\
\text { surface }\end{array}$ & 8.91 & 1.76 & & 14 & 444 & 10 & 29 & 17 & 17 & 0 & 33 & 65 & 98 & 84 & 4.70E-04 \\
\hline CBM12 & horizon 2/8 mix & Fowler 11-18 & gyp & 8.91 & 1.89 & & 14 & 413 & 10 & 24 & 17 & 15 & 0 & 67 & 135 & 202 & 294 & 6.65E-04 \\
\hline $1079-44-1$ & $\begin{array}{c}\text { Leiter A } \\
\text { horizon } 2 / 8 \text { mix }\end{array}$ & Fowler 11-18 & $\begin{array}{c}\mathrm{H} 2 \mathrm{SO} 4 \\
1.68 \\
\mathrm{ml} / \mathrm{gal} \\
\mathrm{H} 2 \mathrm{SO} 4\end{array}$ & 8.94 & 3.88 & 40.5 & 17 & 394 & 8 & 43 & 13 & 7 & 14 & 18 & 21 & 25 & 613 & $1.15 \mathrm{E}-04$ \\
\hline $1079-44-2$ & $\begin{array}{c}\text { Leiter A } \\
\text { horizon } 2 / 8 \text { mix }\end{array}$ & Fowler 11-18 & $\begin{array}{c}1.68 \\
\mathrm{ml} / \mathrm{gal}\end{array}$ & 9.41 & 4.61 & 43 & 15 & 422 & 7 & 42 & 14 & 6 & 0 & 19 & 38 & 57 & 703 & 2.02E-04 \\
\hline
\end{tabular}




\begin{tabular}{|c|c|c|c|c|c|c|c|c|c|c|c|c|c|c|c|c|c|c|}
\hline 1079-44-5 & $\begin{array}{c}\text { Leiter A } \\
\text { horizon } 2 / 8 \text { mix }\end{array}$ & Fowler 11-18 & $\begin{array}{c}\mathrm{H} 2 \mathrm{SO} 4 \\
1.68 \\
\mathrm{ml} / \mathrm{gal} \\
\mathrm{H} 2 \mathrm{SO} 4\end{array}$ & 8.91 & 4.13 & 42.5 & 15 & 406 & 7 & 41 & 14 & 6 & 21 & 17 & 12 & 8 & 668 & $1.29 \mathrm{E}-04$ \\
\hline $1079-44-8$ & $\begin{array}{c}\text { Leiter A } \\
\text { horizon 2/8 mix }\end{array}$ & Fowler 11-18 & $\begin{array}{l}1.68 \\
\mathrm{ml} / \mathrm{gal}\end{array}$ & 9.07 & 3.75 & 40.5 & 13 & 353 & 5 & 36 & 13 & 6 & 0 & 19 & 37 & 56 & 572 & $1.02 \mathrm{E}-04$ \\
\hline $1079-44-3$ & $\begin{array}{l}\text { Leiter A } \\
\text { horizon 2/8 mix } \\
\text { Leiter A }\end{array}$ & Fowler 11-18 & $\begin{array}{l}\text { Gyp } 193 \\
\text { mg/gal } \\
\text { Gyp } 193\end{array}$ & 9.01 & 4.83 & 43.6 & 30 & 572 & 20 & 43 & 16 & 24 & 6 & 25 & 43 & 62 & 18 & $1.00 \mathrm{E}-04$ \\
\hline $1079-44-4$ & $\begin{array}{l}\text { horizon } 2 / 8 \text { mix } \\
\text { Leiter A }\end{array}$ & Fowler 11-18 & $\begin{array}{l}\mathrm{mg} / \mathrm{gal} \\
\text { Gyp } 193\end{array}$ & 9.04 & 4.51 & 41.6 & 30 & 529 & 9 & 37 & 16 & 24 & 7 & 16 & 26 & 35 & 16 & 6.79E-05 \\
\hline $1079-44-6$ & $\begin{array}{c}\text { horizon } 2 / 8 \text { mix } \\
\text { Leiter A }\end{array}$ & Fowler 11-18 & $\begin{array}{l}\text { mg/gal } \\
\text { Gyp } 193\end{array}$ & 8.98 & 4.86 & 40.4 & 30 & 575 & 12 & 52 & 16 & 25 & 7 & 28 & 49 & 71 & 34 & $2.20 \mathrm{E}-04$ \\
\hline $1079-44-7$ & horizon $2 / 8 \mathrm{mix}$ & Fowler 11-18 & $\mathrm{mg} / \mathrm{gal}$ & 9.06 & 4.37 & 40.8 & 30 & 482 & 10 & 43 & 14 & 22 & 0 & 24 & 48 & 71 & 39 & 5.95E-05 \\
\hline CC-14 & $\begin{array}{c}\text { Leiter } \mathrm{Bt} \\
\text { horizon } 2 / 8 \text { mix } \\
\text { Leiter Bt }\end{array}$ & Clear Creek & - & 8.77 & 0.89 & & 37 & 55 & 5 & 87 & 1 & 4 & 0 & 8 & 17 & 25 & 262 & 4.65E-04 \\
\hline CC-15 & $\begin{array}{l}\text { horizon } 2 / 8 \text { mix } \\
\text { Leiter } \mathrm{Bt}\end{array}$ & Clear Creek & - & 8.66 & 0.96 & & 43 & 60 & 4 & 99 & 1 & 4 & 10 & 9 & 9 & 9 & 311 & $5.50 \mathrm{E}-04$ \\
\hline CC-19 & $\begin{array}{l}\text { horizon } 2 / 8 \text { mix } \\
\text { Leiter } \mathrm{Bt}\end{array}$ & Clear Creek & - & 8.79 & 0.86 & & 37 & 55 & 5 & 92 & 1 & 4 & 0 & 8 & 17 & 25 & 260 & $5.20 \mathrm{E}-04$ \\
\hline CC-20 & horizon 2/8 mix & Clear Creek & - & 8.79 & 0.99 & & 45 & 64 & 4 & 96 & 1 & 4 & 0 & 9 & 19 & 28 & 320 & 7.44E-04 \\
\hline CBM-13 & $\begin{array}{c}\text { Leiter Bt } \\
\text { horizon 2/8 mix } \\
\text { Leiter Bt }\end{array}$ & Fowler 11-18 & - & 9.34 & 1.35 & & 8 & 314 & 2 & 10 & 18 & 14 & 0 & 31 & 61 & 92 & 5 & $1.18 \mathrm{E}-04$ \\
\hline CBM-16 & $\begin{array}{l}\text { horizon 2/8 mix } \\
\text { Leiter Bt }\end{array}$ & Fowler 11-18 & - & 9.44 & 1.39 & & 10 & 337 & 3 & 17 & 16 & 15 & 0 & 29 & 58 & 86 & 7 & 2.77E-04 \\
\hline CBM-17 & $\begin{array}{l}\text { horizon } 2 / 8 \mathrm{mix} \\
\text { Leiter } \mathrm{Bt}\end{array}$ & Fowler 11-18 & - & 9.12 & 1.32 & & 10 & 306 & 3 & 12 & 16 & 13 & 1 & 27 & 52 & 78 & 14 & $1.20 \mathrm{E}-04$ \\
\hline CBM-18 & horizon 2/8 mix & Fowler 11-18 & - & 9.3 & 1.29 & & 9 & 312 & 3 & 19 & 15 & 13 & 1 & 23 & 45 & 67 & 6 & $1.28 \mathrm{E}-04$ \\
\hline CBM-21 & $\begin{array}{l}\text { Leiter Bt } \\
\text { horizon } 2 / 8 \text { mix } \\
\text { Leiter Bt }\end{array}$ & Fowler 11-18 & $\begin{array}{l}2.5 \mathrm{t} / \mathrm{ac} \\
\text { surface } \\
\text { gyp } \\
2.5 \mathrm{t} / \mathrm{ac}\end{array}$ & 9 & 1.73 & & 17 & 380 & 5 & 44 & 12 & 9 & 0 & 14 & 28 & 42 & 387 & 4.17E-04 \\
\hline CBM-22 & horizon 2/8 mix & Fowler 11-18 & surface & 9.14 & 1.64 & & 9 & 412 & 6 & 16 & 21 & 17 & 1 & 29 & 57 & 85 & 20 & 8.65E-04 \\
\hline
\end{tabular}




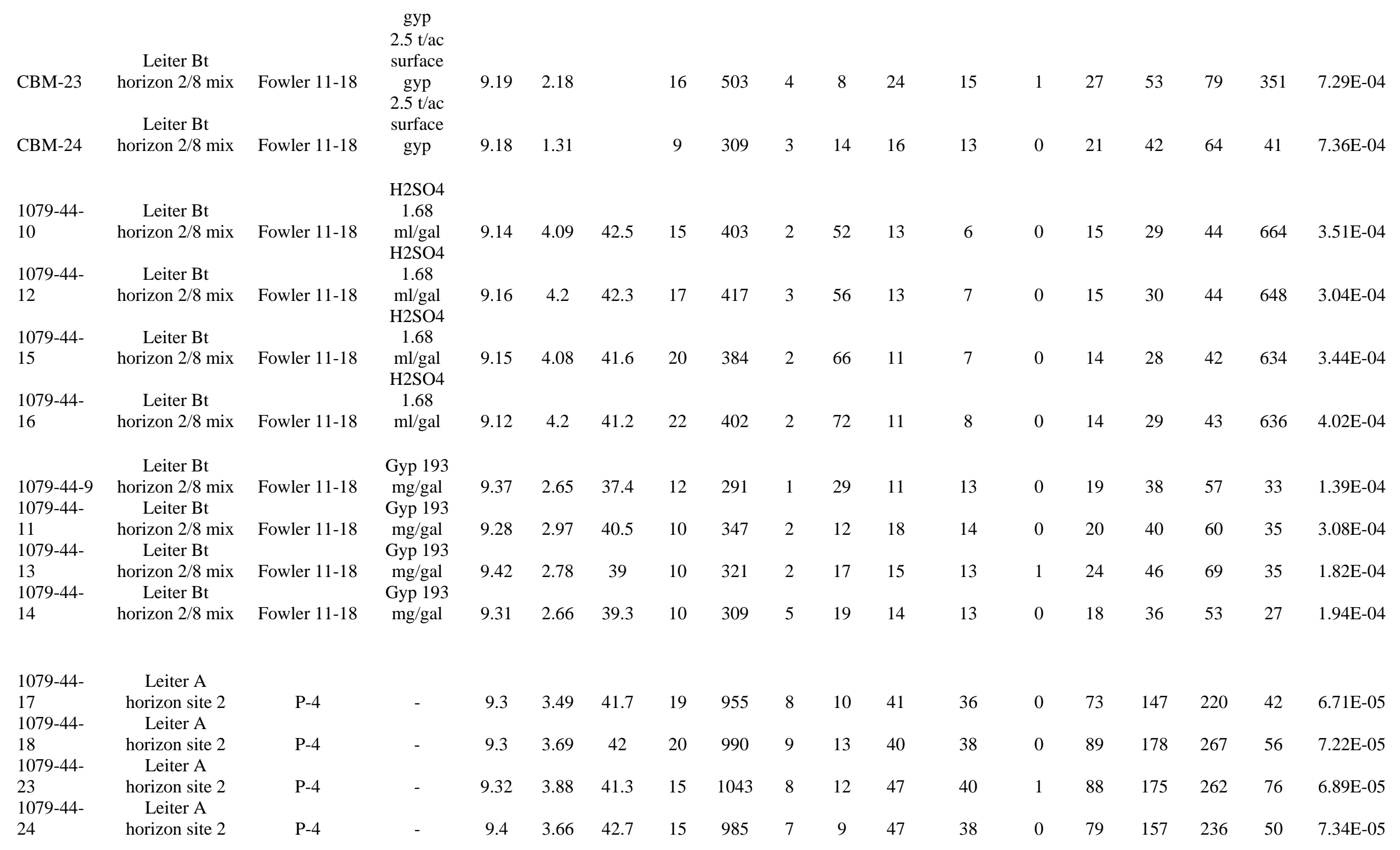




\begin{tabular}{|c|c|c|c|c|c|c|c|c|c|c|c|c|c|c|c|c|c|c|}
\hline 1079-44- & Leiter A & & $\begin{array}{l}4.0 \mathrm{t} / \mathrm{ac} \\
\text { surface }\end{array}$ & & & & & & & & & & & & & & & \\
\hline & horizon site 2 & $\mathrm{P}-4$ & $\begin{array}{c}\text { gyp } \\
4.0 \mathrm{t} / \mathrm{ac}\end{array}$ & 9.03 & 3.4 & 41 & 13 & 863 & 6 & 16 & 39 & 33 & 1 & 70 & 139 & 208 & 147 & $1.59 \mathrm{E}-04$ \\
\hline $\begin{array}{l}1079-44- \\
20\end{array}$ & $\begin{array}{c}\text { Leiter A } \\
\text { horizon site } 2\end{array}$ & P-4 & $\begin{array}{l}\text { surface } \\
\text { gyp } \\
4.0 \mathrm{t} / \mathrm{ac}\end{array}$ & 8.97 & 3.84 & 39.9 & 15 & 979 & 8 & 21 & 40 & 34 & 0 & 63 & 125 & 188 & 258 & 2.35E-04 \\
\hline $\begin{array}{l}1079-44- \\
21\end{array}$ & $\begin{array}{c}\text { Leiter A } \\
\text { horizon site } 2\end{array}$ & P-4 & $\begin{array}{l}\text { surface } \\
\text { gyp } \\
4.0 \mathrm{t} / \mathrm{ac}\end{array}$ & 8.92 & 3.75 & 40.6 & 17 & 938 & 8 & 25 & 36 & 28 & 0 & 66 & 131 & 197 & 500 & $1.92 \mathrm{E}-04$ \\
\hline $\begin{array}{l}1079-44- \\
22\end{array}$ & $\begin{array}{c}\text { Leiter A } \\
\text { horizon site } 2\end{array}$ & P-4 & $\begin{array}{l}\text { surface } \\
\text { gyp }\end{array}$ & 9.12 & 3.85 & 41.7 & 17 & 1072 & 8 & 19 & 43 & 36 & 0 & 62 & 123 & 184 & 313 & $1.64 \mathrm{E}-04$ \\
\hline
\end{tabular}




\title{
Appendix IV
}

\author{
Baseline Soil infiltration Data Collected from the \\ Cooksley Ranch Study Site using the Double Ring Infiltrometer
}


Double Ring Infiltration Data

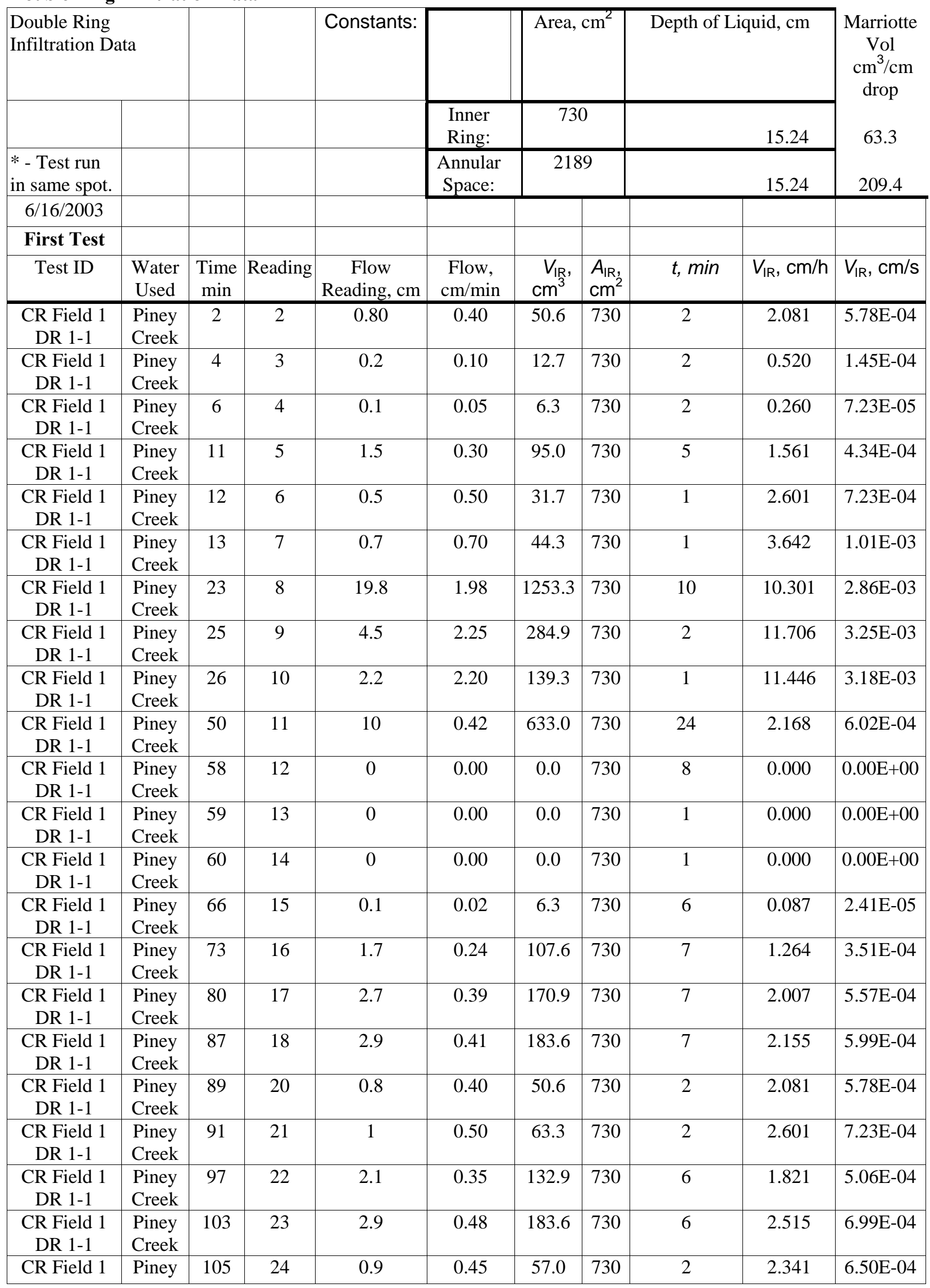




\begin{tabular}{|c|c|c|c|c|c|c|c|c|c|c|}
\hline \multirow[t]{2}{*}{ DR 1-1 } & Creek & & & & & & & \multirow[b]{2}{*}{$\begin{array}{r}\text { Final } \\
\text { Infiltration } \\
\text { Rate: }\end{array}$} & \multirow[b]{2}{*}{2.22} & \multirow[b]{2}{*}{$6.16 \mathrm{E}-04$} \\
\hline & & & & & & & & & & \\
\hline & & & & & & & & & & \\
\hline & & & & & & & & & & \\
\hline \multicolumn{11}{|l|}{$6 / 16 / 2003$} \\
\hline \multicolumn{11}{|l|}{ First Test } \\
\hline Test ID & $\begin{array}{l}\text { Water } \\
\text { Used }\end{array}$ & $\begin{array}{l}\text { Time } \\
\text { min }\end{array}$ & Reading & $\begin{array}{c}\text { Flow } \\
\text { Reading, cm }\end{array}$ & $\begin{array}{l}\text { Flow, } \\
\mathrm{cm} / \mathrm{min}\end{array}$ & $\begin{array}{r}\begin{array}{l}V_{\text {IR }}, \\
\mathrm{cm}^{3}\end{array} \\
\end{array}$ & $\begin{array}{l}A_{\mathrm{IR}} \\
\mathrm{cm}^{2}\end{array}$ & $t, \min$ & $V_{\mathrm{IR}}, \mathrm{cm} / \mathrm{h}$ & $V_{\mathrm{IR}}, \mathrm{cm} / \mathrm{s}$ \\
\hline $\begin{array}{c}\text { CR Field } 1 \\
\text { DR 2-1 }\end{array}$ & $\begin{array}{l}\text { Piney } \\
\text { Creek }\end{array}$ & 1 & 2 & 6.60 & 6.60 & 417.8 & 730 & 1 & 34.338 & $9.54 \mathrm{E}-03$ \\
\hline $\begin{array}{c}\text { CR Field } 1 \\
\text { DR 2-1 }\end{array}$ & $\begin{array}{l}\text { Piney } \\
\text { Creek }\end{array}$ & 2 & 3 & 1.9 & 1.90 & 120.3 & 731 & 1 & 9.872 & $2.74 \mathrm{E}-03$ \\
\hline $\begin{array}{c}\text { CR Field } 1 \\
\text { DR 2-1 }\end{array}$ & $\begin{array}{l}\text { Piney } \\
\text { Creek }\end{array}$ & 3 & 4 & 1.7 & 1.70 & 107.6 & 732 & 1 & 8.820 & $2.45 \mathrm{E}-03$ \\
\hline $\begin{array}{c}\text { CR Field } 1 \\
\text { DR 2-1 }\end{array}$ & $\begin{array}{l}\text { Piney } \\
\text { Creek }\end{array}$ & 4 & 5 & 1.6 & 1.60 & 101.3 & 733 & 1 & 8.290 & $2.30 \mathrm{E}-03$ \\
\hline $\begin{array}{c}\text { CR Field } 1 \\
\text { DR 2-1 }\end{array}$ & $\begin{array}{l}\text { Piney } \\
\text { Creek }\end{array}$ & 5 & 6 & 4.7 & 4.70 & 297.5 & 734 & 1 & 24.320 & 6.76E-03 \\
\hline $\begin{array}{c}\text { CR Field } 1 \\
\text { DR 2-1 }\end{array}$ & $\begin{array}{l}\text { Piney } \\
\text { Creek }\end{array}$ & 10 & 7 & 19.7 & 3.94 & 1247.0 & 735 & 5 & 20.359 & 5.66E-03 \\
\hline $\begin{array}{c}\text { CR Field } 1 \\
\text { DR 2-1 }\end{array}$ & $\begin{array}{l}\text { Piney } \\
\text { Creek }\end{array}$ & 13 & 8 & 12.6 & 4.20 & 797.6 & 736 & 3 & 21.673 & $6.02 \mathrm{E}-03$ \\
\hline $\begin{array}{c}\text { CR Field } 1 \\
\text { DR 2-1 }\end{array}$ & $\begin{array}{l}\text { Piney } \\
\text { Creek }\end{array}$ & 14 & 9 & 1.2 & 1.20 & 76.0 & 737 & 1 & 6.184 & $1.72 \mathrm{E}-03$ \\
\hline $\begin{array}{c}\text { CR Field } 1 \\
\text { DR 2-1 }\end{array}$ & $\begin{array}{l}\text { Piney } \\
\text { Creek }\end{array}$ & 15 & 10 & 1.3 & 1.30 & 82.3 & 738 & 1 & 6.690 & $1.86 \mathrm{E}-03$ \\
\hline $\begin{array}{c}\text { CR Field } 1 \\
\text { DR 2-1 }\end{array}$ & $\begin{array}{l}\text { Piney } \\
\text { Creek }\end{array}$ & 17 & 11 & 2.9 & 1.45 & 183.6 & 739 & 2 & 7.452 & $2.07 \mathrm{E}-03$ \\
\hline $\begin{array}{c}\text { CR Field } 1 \\
\text { DR 2-1 }\end{array}$ & $\begin{array}{l}\text { Piney } \\
\text { Creek }\end{array}$ & 21 & 12 & 9 & 2.25 & 569.7 & 740 & 4 & 11.548 & $3.21 \mathrm{E}-03$ \\
\hline $\begin{array}{c}\text { CR Field } 1 \\
\text { DR 2-1 }\end{array}$ & $\begin{array}{l}\text { Piney } \\
\text { Creek }\end{array}$ & 23 & 13 & 11.4 & 5.70 & 721.6 & 741 & 2 & 29.215 & 8.12E-03 \\
\hline $\begin{array}{c}\text { CR Field } 1 \\
\text { DR 2-1 }\end{array}$ & $\begin{array}{l}\text { Piney } \\
\text { Creek }\end{array}$ & 24 & 14 & 4.4 & 4.40 & 278.5 & 742 & 1 & 22.522 & $6.26 \mathrm{E}-03$ \\
\hline $\begin{array}{c}\text { CR Field } 1 \\
\text { DR 2-1 }\end{array}$ & $\begin{array}{l}\text { Piney } \\
\text { Creek }\end{array}$ & 25 & 15 & 3.1 & 3.10 & 196.2 & 743 & 1 & 15.846 & $4.40 \mathrm{E}-03$ \\
\hline $\begin{array}{c}\text { CR Field } 1 \\
\text { DR 2-1 }\end{array}$ & $\begin{array}{l}\text { Piney } \\
\text { Creek }\end{array}$ & 27 & 16 & 7.2 & 3.60 & 455.8 & 744 & 2 & 18.377 & $5.10 \mathrm{E}-03$ \\
\hline $\begin{array}{c}\text { CR Field } 1 \\
\text { DR 2-1 }\end{array}$ & $\begin{array}{l}\text { Piney } \\
\text { Creek }\end{array}$ & 28 & 17 & 2 & 2.00 & 126.6 & 745 & 1 & 10.196 & 2.83E-03 \\
\hline $\begin{array}{c}\text { CR Field } 1 \\
\text { DR 2-1 }\end{array}$ & $\begin{array}{l}\text { Piney } \\
\text { Creek }\end{array}$ & 36 & 18 & 18.3 & 2.29 & 1158.4 & 746 & 8 & 11.646 & $3.24 \mathrm{E}-03$ \\
\hline $\begin{array}{c}\text { CR Field } 1 \\
\text { DR 2-1 }\end{array}$ & $\begin{array}{l}\text { Piney } \\
\text { Creek }\end{array}$ & 39 & 19 & 6 & 2.00 & 379.8 & 747 & 3 & 10.169 & 2.82E-03 \\
\hline $\begin{array}{c}\text { CR Field } 1 \\
\text { DR 2-1 }\end{array}$ & $\begin{array}{l}\text { Piney } \\
\text { Creek }\end{array}$ & 40 & 20 & 0.9 & 0.90 & 57.0 & 748 & 1 & 4.570 & $1.27 \mathrm{E}-03$ \\
\hline $\begin{array}{c}\text { CR Field } 1 \\
\text { DR 2-1 }\end{array}$ & $\begin{array}{l}\text { Piney } \\
\text { Creek }\end{array}$ & 41 & 21 & 0.9 & 0.90 & 57.0 & 749 & 1 & 4.564 & $1.27 \mathrm{E}-03$ \\
\hline $\begin{array}{c}\text { CR Field } 1 \\
\text { DR 2-1 }\end{array}$ & $\begin{array}{l}\text { Piney } \\
\text { Creek }\end{array}$ & 51 & 22 & 12.7 & 1.27 & 803.9 & 750 & 10 & 6.431 & $1.79 \mathrm{E}-03$ \\
\hline $\begin{array}{c}\text { CR Field } 1 \\
\text { DR 2-1 }\end{array}$ & $\begin{array}{l}\text { Piney } \\
\text { Creek }\end{array}$ & 58 & 23 & 15.5 & 2.21 & 981.2 & 751 & 7 & 11.198 & $3.11 \mathrm{E}-03$ \\
\hline $\begin{array}{c}\text { CR Field } 1 \\
\text { DR 2-1 }\end{array}$ & $\begin{array}{l}\text { Piney } \\
\text { Creek }\end{array}$ & 59 & 24 & 0.8 & 0.80 & 50.6 & 752 & 1 & 4.040 & $1.12 \mathrm{E}-03$ \\
\hline
\end{tabular}




\begin{tabular}{|c|c|c|c|c|c|c|c|c|c|c|}
\hline $\begin{array}{c}\text { CR Field } 1 \\
\text { DR 2-1 }\end{array}$ & $\begin{array}{l}\text { Piney } \\
\text { Creek }\end{array}$ & 60 & 25 & 1.9 & 1.90 & 120.3 & 753 & 1 & 9.583 & $2.66 \mathrm{E}-03$ \\
\hline $\begin{array}{c}\text { CR Field } 1 \\
\text { DR 2-1 }\end{array}$ & $\begin{array}{l}\text { Piney } \\
\text { Creek }\end{array}$ & 70 & 26 & 12.1 & 1.21 & 765.9 & 754 & 10 & 6.095 & $1.69 \mathrm{E}-03$ \\
\hline $\begin{array}{c}\text { CR Field } 1 \\
\text { DR 2-1 }\end{array}$ & $\begin{array}{l}\text { Piney } \\
\text { Creek }\end{array}$ & 75 & 27 & 5.4 & 1.08 & 341.8 & 755 & 5 & 5.433 & $1.51 \mathrm{E}-03$ \\
\hline $\begin{array}{c}\text { CR Field } 1 \\
\text { DR 2-1 }\end{array}$ & $\begin{array}{l}\text { Piney } \\
\text { Creek }\end{array}$ & 78 & 28 & 3 & 1.00 & 189.9 & 756 & 3 & 5.024 & $1.40 \mathrm{E}-03$ \\
\hline $\begin{array}{c}\text { CR Field } 1 \\
\text { DR 2-1 }\end{array}$ & $\begin{array}{l}\text { Piney } \\
\text { Creek }\end{array}$ & 79 & 29 & 1.5 & 1.50 & 95.0 & 757 & 1 & 7.526 & $2.09 \mathrm{E}-03$ \\
\hline $\begin{array}{c}\text { CR Field } 1 \\
\text { DR 2-1 }\end{array}$ & $\begin{array}{l}\text { Piney } \\
\text { Creek }\end{array}$ & 84 & 30 & 5.3 & 1.06 & 335.5 & 758 & 5 & 5.311 & $1.48 \mathrm{E}-03$ \\
\hline $\begin{array}{c}\text { CR Field } 1 \\
\text { DR 2-1 }\end{array}$ & $\begin{array}{l}\text { Piney } \\
\text { Creek }\end{array}$ & 89 & 31 & 5.8 & 1.16 & 367.1 & 759 & 5 & 5.805 & $1.61 \mathrm{E}-03$ \\
\hline $\begin{array}{c}\text { CR Field } 1 \\
\text { DR 2-1 }\end{array}$ & $\begin{array}{l}\text { Piney } \\
\text { Creek }\end{array}$ & 94 & 32 & 5.9 & 1.18 & 373.5 & 760 & 5 & 5.897 & $1.64 \mathrm{E}-03$ \\
\hline $\begin{array}{c}\text { CR Field } 1 \\
\text { DR 2-1 }\end{array}$ & $\begin{array}{l}\text { Piney } \\
\text { Creek }\end{array}$ & 99 & 33 & 6 & 1.20 & 379.8 & 761 & 5 & 5.989 & $1.66 \mathrm{E}-03$ \\
\hline $\begin{array}{c}\text { CR Field } 1 \\
\text { DR 2-1 }\end{array}$ & $\begin{array}{l}\text { Piney } \\
\text { Creek }\end{array}$ & 101 & 34 & 1 & 0.50 & 63.3 & 762 & 2 & 2.492 & $6.92 \mathrm{E}-04$ \\
\hline $\begin{array}{c}\text { CR Field } 1 \\
\text { DR 2-1 }\end{array}$ & $\begin{array}{l}\text { Piney } \\
\text { Creek }\end{array}$ & 109 & 35 & 7.1 & 0.89 & 449.4 & 763 & 8 & 4.418 & $1.23 \mathrm{E}-03$ \\
\hline $\begin{array}{c}\text { CR Field } 1 \\
\text { DR 2-1 }\end{array}$ & $\begin{array}{l}\text { Piney } \\
\text { Creek }\end{array}$ & 115 & 36 & 6.5 & 1.08 & 411.5 & 764 & 6 & 5.385 & $1.50 \mathrm{E}-03$ \\
\hline $\begin{array}{c}\text { CR Field } 1 \\
\text { DR 2-1 }\end{array}$ & $\begin{array}{l}\text { Piney } \\
\text { Creek }\end{array}$ & 122 & 37 & 5.5 & 0.79 & 348.2 & 765 & 7 & 3.901 & $1.08 \mathrm{E}-03$ \\
\hline $\begin{array}{c}\text { CR Field } 1 \\
\text { DR 2-1 }\end{array}$ & $\begin{array}{l}\text { Piney } \\
\text { Creek }\end{array}$ & 128 & 38 & 5.3 & 0.88 & 335.5 & 766 & 6 & 4.380 & $1.22 \mathrm{E}-03$ \\
\hline $\begin{array}{c}\text { CR Field } 1 \\
\text { DR 2-1 }\end{array}$ & $\begin{array}{l}\text { Piney } \\
\text { Creek }\end{array}$ & 134 & 39 & 4.2 & 0.70 & 265.9 & 767 & 6 & 3.466 & $9.63 \mathrm{E}-04$ \\
\hline \multirow[t]{2}{*}{$\begin{array}{c}\text { CR Field } 1 \\
\text { DR 2-1 }\end{array}$} & $\begin{array}{l}\text { Piney } \\
\text { Creek }\end{array}$ & 142 & 40 & 6.2 & 0.78 & 392.5 & 768 & 8 & 3.833 & $1.06 \mathrm{E}-03$ \\
\hline & & & & & & & & $\begin{array}{r}\text { Final } \\
\text { Infiltration } \\
\text { Rate: }\end{array}$ & 3.89 & $1.08 \mathrm{E}-03$ \\
\hline & & & & & & & & & & \\
\hline \multicolumn{11}{|l|}{ 6/17/2003 } \\
\hline \multicolumn{11}{|l|}{\begin{tabular}{|l} 
2nd Test- \\
Same \\
location \\
\end{tabular}} \\
\hline Test ID & $\begin{array}{l}\text { Water } \\
\text { Used }\end{array}$ & $\begin{array}{l}\text { Time } \\
\text { min }\end{array}$ & Reading & $\begin{array}{c}\text { Flow } \\
\text { Reading, cm } \\
\end{array}$ & $\begin{array}{l}\text { Flow, } \\
\mathrm{cm} / \mathrm{min}\end{array}$ & $\begin{array}{r}\begin{array}{r}V_{\mathrm{IR}}, \\
\mathrm{cm}^{3}\end{array} \\
\end{array}$ & $\begin{array}{l}A_{\mathrm{IR}} \\
\mathrm{cm}^{2}\end{array}$ & $t, \min$ & $V_{\mathrm{IR}}, \mathrm{cm} / \mathrm{h}$ & $V_{\mathrm{IR}}, \mathrm{cm} / \mathrm{s}$ \\
\hline $\begin{array}{c}\text { CR Field } 1 \\
\text { DR 1-2 }\end{array}$ & $\begin{array}{l}\text { Piney } \\
\text { Creek }\end{array}$ & 2 & 2 & 0.10 & 0.10 & 6.3 & 725 & 1 & 0.524 & $1.46 \mathrm{E}-04$ \\
\hline $\begin{array}{c}\text { CR Field } 1 \\
\text { DR 1-2 }\end{array}$ & $\begin{array}{l}\text { Piney } \\
\text { Creek }\end{array}$ & 3 & 3 & 0.1 & 0.10 & 6.3 & 725 & 1 & 0.524 & $1.46 \mathrm{E}-04$ \\
\hline $\begin{array}{c}\text { CR Field } 1 \\
\text { DR 1-2 }\end{array}$ & $\begin{array}{l}\text { Piney } \\
\text { Creek }\end{array}$ & 13 & 4 & 0.2 & 0.02 & 12.7 & 725 & 10 & 0.105 & $2.91 \mathrm{E}-05$ \\
\hline $\begin{array}{c}\text { CR Field } 1 \\
\text { DR 1-2 }\end{array}$ & $\begin{array}{l}\text { Piney } \\
\text { Creek }\end{array}$ & 24 & 5 & 4 & 0.36 & 253.2 & 725 & 11 & 1.905 & $5.29 \mathrm{E}-04$ \\
\hline $\begin{array}{c}\text { CR Field } 1 \\
\text { DR 1-2 }\end{array}$ & $\begin{array}{l}\text { Piney } \\
\text { Creek }\end{array}$ & 34 & 6 & 3.8 & 0.38 & 240.5 & 725 & 10 & 1.991 & $5.53 \mathrm{E}-04$ \\
\hline $\begin{array}{c}\text { CR Field } 1 \\
\text { DR 1-2 }\end{array}$ & $\begin{array}{l}\text { Piney } \\
\text { Creek }\end{array}$ & 44 & 7 & 2.8 & 0.28 & 177.2 & 725 & 10 & 1.467 & 4.07E-04 \\
\hline CR Field 1 & Piney & 47 & 8 & 5.2 & 1.73 & 329.2 & 725 & 3 & 9.080 & $2.52 \mathrm{E}-03$ \\
\hline
\end{tabular}




\begin{tabular}{|c|c|c|c|c|c|c|c|c|c|c|}
\hline DR 1-2 & Creek & & & & & & & & & \\
\hline $\begin{array}{c}\text { CR Field } 1 \\
\text { DR 1-2 }\end{array}$ & $\begin{array}{l}\text { Piney } \\
\text { Creek }\end{array}$ & 49 & 9 & 5.1 & 2.55 & 322.8 & 725 & 2 & 13.358 & $3.71 \mathrm{E}-03$ \\
\hline $\begin{array}{c}\text { CR Field } 1 \\
\text { DR 1-2 }\end{array}$ & $\begin{array}{l}\text { Piney } \\
\text { Creek }\end{array}$ & 56 & 10 & 2.5 & 0.36 & 158.3 & 725 & 7 & 1.871 & $5.20 \mathrm{E}-04$ \\
\hline $\begin{array}{c}\text { CR Field } 1 \\
\text { DR 1-2 }\end{array}$ & $\begin{array}{l}\text { Piney } \\
\text { Creek }\end{array}$ & 69 & 11 & 3.6 & 0.28 & 227.9 & 725 & 13 & 1.451 & $4.03 \mathrm{E}-04$ \\
\hline $\begin{array}{c}\text { CR Field } 1 \\
\text { DR 1-2 }\end{array}$ & $\begin{array}{l}\text { Piney } \\
\text { Creek }\end{array}$ & 83 & 12 & 2.4 & 0.17 & 151.9 & 725 & 14 & 0.898 & $2.49 \mathrm{E}-04$ \\
\hline $\begin{array}{c}\text { CR Field } 1 \\
\text { DR 1-2 }\end{array}$ & $\begin{array}{l}\text { Piney } \\
\text { Creek }\end{array}$ & 98 & 13 & 6 & 0.40 & 379.8 & 725 & 15 & 2.095 & $5.82 \mathrm{E}-04$ \\
\hline $\begin{array}{c}\text { CR Field } 1 \\
\text { DR 1-2 }\end{array}$ & $\begin{array}{l}\text { Piney } \\
\text { Creek }\end{array}$ & 113 & 14 & 2.2 & 0.15 & 139.3 & 725 & 15 & 0.768 & $2.13 \mathrm{E}-04$ \\
\hline $\begin{array}{c}\text { CR Field } 1 \\
\text { DR 1-2 }\end{array}$ & $\begin{array}{l}\text { Piney } \\
\text { Creek }\end{array}$ & 125 & 16 & 1.8 & 0.15 & 113.9 & 725 & 12 & 0.786 & $2.18 \mathrm{E}-04$ \\
\hline $\begin{array}{c}\text { CR Field } 1 \\
\text { DR 1-2 }\end{array}$ & $\begin{array}{l}\text { Piney } \\
\text { Creek }\end{array}$ & 127 & 17 & 0.3 & 0.15 & 19.0 & 725 & 2 & 0.786 & $2.18 \mathrm{E}-04$ \\
\hline $\begin{array}{c}\text { CR Field } 1 \\
\text { DR 1-2 }\end{array}$ & $\begin{array}{l}\text { Piney } \\
\text { Creek }\end{array}$ & 131 & 18 & 0.6 & 0.15 & 38.0 & 725 & 4 & 0.786 & $2.18 \mathrm{E}-04$ \\
\hline & & & & & & & & $\begin{array}{r}\text { Final } \\
\text { Infiltration } \\
\text { Rate: } \\
\end{array}$ & 1.08 & 3.00E-04 \\
\hline & & & & & & & & & & \\
\hline & & & & & & & & & & \\
\hline 6/17/2003 & & & & & & & & & & \\
\hline \multicolumn{11}{|l|}{$\begin{array}{l}\text { 2nd Test- } \\
\text { Same } \\
\text { location } \\
\end{array}$} \\
\hline Test ID & $\begin{array}{l}\text { Water } \\
\text { Used }\end{array}$ & $\begin{array}{l}\text { Time } \\
\text { min }\end{array}$ & Reading & $\begin{array}{c}\text { Flow } \\
\text { Reading, cm }\end{array}$ & $\begin{array}{l}\text { Flow, } \\
\mathrm{cm} / \mathrm{min}\end{array}$ & $\begin{array}{l}\begin{array}{l}V_{\mathrm{IR}}, \\
\mathrm{cm}^{3}\end{array}\end{array}$ & $\begin{array}{l}A_{\mathrm{IR}} \\
\mathrm{cm}^{2}\end{array}$ & $t, \min$ & $V_{\mathrm{IR}}, \mathrm{cm} / \mathrm{h}$ & $V_{\mathrm{IR}}, \mathrm{cm} / \mathrm{s}$ \\
\hline $\begin{array}{c}\text { CR Field } 1 \\
\text { DR 2-2 }\end{array}$ & $\begin{array}{c}\text { CBM- } \\
\text { CR }\end{array}$ & 1 & 2 & 0.90 & 0.90 & 57.0 & 725 & 1 & 4.715 & $1.31 \mathrm{E}-03$ \\
\hline $\begin{array}{c}\text { CR Field } 1 \\
\text { DR 2-2 }\end{array}$ & $\begin{array}{c}\text { CBM- } \\
\text { CR }\end{array}$ & 2 & 3 & 1 & 1.00 & 63.3 & 725 & 1 & 5.239 & $1.46 \mathrm{E}-03$ \\
\hline $\begin{array}{c}\text { CR Field } 1 \\
\text { DR 2-2 }\end{array}$ & $\begin{array}{c}\text { CBM- } \\
\text { CR }\end{array}$ & 3 & 4 & 0.9 & 0.90 & 57.0 & 725 & 1 & 4.715 & $1.31 \mathrm{E}-03$ \\
\hline $\begin{array}{c}\text { CR Field } 1 \\
\text { DR 2-2 }\end{array}$ & $\begin{array}{c}\text { CBM- } \\
\text { CR }\end{array}$ & 8 & 5 & 3.2 & 0.64 & 202.6 & 725 & 5 & 3.353 & $9.31 \mathrm{E}-04$ \\
\hline $\begin{array}{c}\text { CR Field } 1 \\
\text { DR 2-2 }\end{array}$ & $\begin{array}{c}\text { CBM- } \\
\text { CR }\end{array}$ & 15 & 6 & 10.9 & 1.56 & 690.0 & 725 & 7 & 8.157 & $2.27 \mathrm{E}-03$ \\
\hline $\begin{array}{c}\text { CR Field } 1 \\
\text { DR 2-2 }\end{array}$ & $\begin{array}{c}\text { CBM- } \\
\text { CR }\end{array}$ & 25 & 7 & 10 & 1.00 & 633.0 & 725 & 10 & 5.239 & $1.46 \mathrm{E}-03$ \\
\hline $\begin{array}{c}\text { CR Field } 1 \\
\text { DR 2-2 }\end{array}$ & $\begin{array}{c}\text { CBM- } \\
\text { CR }\end{array}$ & 35 & 8 & 5.5 & 0.55 & 348.2 & 725 & 10 & 2.881 & $8.00 \mathrm{E}-04$ \\
\hline $\begin{array}{c}\text { CR Field } 1 \\
\text { DR 2-2 }\end{array}$ & $\begin{array}{c}\text { CBM- } \\
\text { CR }\end{array}$ & 45 & 9 & 8.7 & 0.87 & 550.7 & 725 & 10 & 4.558 & $1.27 \mathrm{E}-03$ \\
\hline $\begin{array}{c}\text { CR Field } 1 \\
\text { DR 2-2 }\end{array}$ & $\begin{array}{c}\text { CBM- } \\
\text { CR }\end{array}$ & 48 & 10 & 2.9 & 0.97 & 183.6 & 725 & 3 & 5.064 & $1.41 \mathrm{E}-03$ \\
\hline $\begin{array}{c}\text { CR Field } 1 \\
\text { DR 2-2 }\end{array}$ & $\begin{array}{c}\text { CBM- } \\
\text { CR }\end{array}$ & 49 & 11 & 0.7 & 0.70 & 44.3 & 725 & 1 & 3.667 & $1.02 \mathrm{E}-03$ \\
\hline $\begin{array}{c}\text { CR Field } 1 \\
\text { DR 2-2 }\end{array}$ & $\begin{array}{c}\text { CBM- } \\
\text { CR }\end{array}$ & 50 & 12 & 0.5 & 0.50 & 31.7 & 725 & 1 & 2.619 & 7.28E-04 \\
\hline $\begin{array}{c}\text { CR Field } 1 \\
\text { DR 2-2 }\end{array}$ & $\begin{array}{c}\text { CBM- } \\
\text { CR }\end{array}$ & 51 & 13 & 0.4 & 0.40 & 25.3 & 725 & 1 & 2.095 & 5.82E-04 \\
\hline $\begin{array}{c}\text { CR Field } 1 \\
\text { DR 2-2 }\end{array}$ & $\begin{array}{c}\text { CBM- } \\
\text { CR }\end{array}$ & 56 & 14 & 2 & 0.40 & 126.6 & 725 & 5 & 2.095 & 5.82E-04 \\
\hline
\end{tabular}




\begin{tabular}{|c|c|c|c|c|c|c|c|c|c|c|}
\hline $\begin{array}{c}\text { CR Field } 1 \\
\text { DR 2-2 }\end{array}$ & $\begin{array}{c}\text { CBM- } \\
\text { CR }\end{array}$ & 61 & 15 & 1.9 & 0.38 & 120.3 & 725 & 5 & 1.991 & 5.53E-04 \\
\hline & & & & & & & & $\begin{array}{r}\text { Final } \\
\text { Infiltration } \\
\text { Rate: }\end{array}$ & 2.06 & 5.72E-04 \\
\hline & & & & & & & & & & \\
\hline & & & & & & & & & & \\
\hline \multicolumn{11}{|l|}{$6 / 17 / 2003$} \\
\hline \multicolumn{11}{|l|}{ First Test } \\
\hline Test ID & $\begin{array}{l}\text { Water } \\
\text { Used }\end{array}$ & $\begin{array}{l}\text { Time } \\
\text { min }\end{array}$ & Reading & $\begin{array}{c}\text { Flow } \\
\text { Reading, cm }\end{array}$ & $\begin{array}{l}\text { Flow, } \\
\mathrm{cm} / \mathrm{min}\end{array}$ & $\begin{array}{r}\begin{array}{r}V_{\mathrm{IR}}, \\
\mathrm{cm}^{3}\end{array} \\
\end{array}$ & $\begin{array}{l}A_{\mathrm{IR}} \\
\mathrm{cm}^{2}\end{array}$ & $t, \min$ & $V_{\mathrm{IR}}, \mathrm{cm} / \mathrm{h}$ & $V_{\mathrm{IR}}, \mathrm{cm} / \mathrm{s}$ \\
\hline $\begin{array}{c}\text { CR Field } 1 \\
\text { DR 3-1 }\end{array}$ & $\begin{array}{l}\text { Piney } \\
\text { Creek }\end{array}$ & 1 & 2 & 20.50 & 20.50 & 1297.7 & 730 & 1 & 106.656 & $2.96 \mathrm{E}-02$ \\
\hline $\begin{array}{c}\text { CR Field } 1 \\
\text { DR 3-1 }\end{array}$ & $\begin{array}{l}\text { Piney } \\
\text { Creek }\end{array}$ & 3 & 3 & 16.5 & 8.25 & 1044.5 & 730 & 2 & 42.923 & 1.19E-02 \\
\hline $\begin{array}{c}\text { CR Field } 1 \\
\text { DR 3-1 }\end{array}$ & $\begin{array}{l}\text { Piney } \\
\text { Creek }\end{array}$ & 5 & 4 & 14.5 & 7.25 & 917.9 & 730 & 2 & 37.720 & 1.05E-02 \\
\hline $\begin{array}{c}\text { CR Field } 1 \\
\text { DR 3-1 }\end{array}$ & $\begin{array}{l}\text { Piney } \\
\text { Creek }\end{array}$ & 9 & 5 & 11.7 & 2.93 & 740.6 & 730 & 4 & 15.218 & $4.23 \mathrm{E}-03$ \\
\hline $\begin{array}{c}\text { CR Field } 1 \\
\text { DR 3-1 }\end{array}$ & $\begin{array}{l}\text { Piney } \\
\text { Creek }\end{array}$ & 14 & 6 & 17.8 & 3.56 & 1126.7 & 730 & 5 & 18.522 & $5.14 \mathrm{E}-03$ \\
\hline $\begin{array}{c}\text { CR Field } 1 \\
\text { DR 3-1 }\end{array}$ & $\begin{array}{l}\text { Piney } \\
\text { Creek }\end{array}$ & 19 & 7 & 14.1 & 2.82 & 892.5 & 730 & 5 & 14.672 & $4.08 \mathrm{E}-03$ \\
\hline $\begin{array}{c}\text { CR Field } 1 \\
\text { DR 3-1 }\end{array}$ & $\begin{array}{l}\text { Piney } \\
\text { Creek }\end{array}$ & 21 & 8 & 2.9 & 1.45 & 183.6 & 730 & 2 & 7.544 & $2.10 \mathrm{E}-03$ \\
\hline $\begin{array}{c}\text { CR Field } 1 \\
\text { DR 3-1 }\end{array}$ & $\begin{array}{l}\text { Piney } \\
\text { Creek }\end{array}$ & 27 & 9 & 14.7 & 2.45 & 930.5 & 730 & 6 & 12.747 & $3.54 \mathrm{E}-03$ \\
\hline $\begin{array}{c}\text { CR Field } 1 \\
\text { DR 3-1 }\end{array}$ & $\begin{array}{l}\text { Piney } \\
\text { Creek }\end{array}$ & 32 & 10 & 14.7 & 2.94 & 930.5 & 730 & 5 & 15.296 & $4.25 \mathrm{E}-03$ \\
\hline $\begin{array}{c}\text { CR Field } 1 \\
\text { DR 3-1 }\end{array}$ & $\begin{array}{l}\text { Piney } \\
\text { Creek }\end{array}$ & 37 & 11 & 6.8 & 1.36 & 430.4 & 730 & 5 & 7.076 & 1.97E-03 \\
\hline $\begin{array}{c}\text { CR Field } 1 \\
\text { DR 3-1 }\end{array}$ & $\begin{array}{l}\text { Piney } \\
\text { Creek }\end{array}$ & 42 & 12 & 6.4 & 1.28 & 405.1 & 730 & 5 & 6.660 & $1.85 \mathrm{E}-03$ \\
\hline $\begin{array}{c}\text { CR Field } 1 \\
\text { DR 3-1 }\end{array}$ & $\begin{array}{l}\text { Piney } \\
\text { Creek }\end{array}$ & 46 & 13 & 1.7 & 0.43 & 107.6 & 730 & 4 & 2.211 & $6.14 \mathrm{E}-04$ \\
\hline $\begin{array}{c}\text { CR Field } 1 \\
\text { DR 3-1 }\end{array}$ & $\begin{array}{l}\text { Piney } \\
\text { Creek }\end{array}$ & 49 & 14 & 9.5 & 3.17 & 601.4 & 730 & 3 & 16.475 & $4.58 \mathrm{E}-03$ \\
\hline $\begin{array}{c}\text { CR Field } 1 \\
\text { DR 3-1 }\end{array}$ & $\begin{array}{l}\text { Piney } \\
\text { Creek }\end{array}$ & 54 & 15 & 7 & 1.40 & 443.1 & 730 & 5 & 7.284 & $2.02 \mathrm{E}-03$ \\
\hline $\begin{array}{c}\text { CR Field } 1 \\
\text { DR 3-1 }\end{array}$ & $\begin{array}{l}\text { Piney } \\
\text { Creek }\end{array}$ & 59 & 16 & 6.7 & 1.34 & 424.1 & 730 & 5 & 6.972 & 1.94E-03 \\
\hline \multirow[t]{2}{*}{$\begin{array}{c}\text { CR Field } 1 \\
\text { DR 3-1 }\end{array}$} & $\begin{array}{l}\text { Piney } \\
\text { Creek }\end{array}$ & 61 & 17 & 2.7 & 1.35 & 170.9 & 730 & 2 & 7.024 & $1.95 \mathrm{E}-03$ \\
\hline & & & & & & & & $\begin{array}{r}\text { Final } \\
\text { Infiltration } \\
\text { Rate: }\end{array}$ & 7.09 & 1.97E-03 \\
\hline & & & & & & & & & & \\
\hline & & & & & & & & & & \\
\hline \multicolumn{11}{|l|}{ 6/17/2003 } \\
\hline \multicolumn{11}{|l|}{ First Test } \\
\hline Test ID & $\begin{array}{l}\text { Water } \\
\text { Used }\end{array}$ & $\begin{array}{l}\text { Time } \\
\text { min }\end{array}$ & Reading & $\begin{array}{c}\text { Flow } \\
\text { Reading, cm }\end{array}$ & $\begin{array}{l}\text { Flow, } \\
\mathrm{cm} / \mathrm{min}\end{array}$ & $\begin{array}{r}\begin{array}{l}V_{\mathrm{IR}}, \\
\mathrm{cm}^{3}\end{array} \\
\end{array}$ & $\begin{array}{l}A_{\mathrm{IR}} \\
\mathrm{cm}^{2} \\
\end{array}$ & $t, \min$ & $V_{\mathrm{IR}}, \mathrm{cm} / \mathrm{h}$ & $V_{\mathrm{IR}}, \mathrm{cm} / \mathrm{s}$ \\
\hline $\begin{array}{c}\text { CR Field } 1 \\
\text { DR 4-1 }\end{array}$ & $\begin{array}{l}\text { Piney } \\
\text { Creek }\end{array}$ & 1 & 2 & 14.20 & 14.20 & 898.9 & 730 & 1 & 73.879 & $2.05 \mathrm{E}-02$ \\
\hline
\end{tabular}




\begin{tabular}{|c|c|c|c|c|c|c|c|c|c|c|}
\hline $\begin{array}{l}\text { CR Field } 1 \\
\text { DR 4-1 }\end{array}$ & $\begin{array}{l}\text { Piney } \\
\text { Creek }\end{array}$ & 2 & 3 & 13.5 & 13.50 & 854.6 & 730 & 1 & 70.237 & 1.95E-02 \\
\hline $\begin{array}{c}\text { CR Field } 1 \\
\text { DR 4-1 }\end{array}$ & $\begin{array}{l}\text { Piney } \\
\text { Creek }\end{array}$ & 3 & 4 & 5.5 & 5.50 & 348.2 & 730 & 1 & 28.615 & 7.95E-03 \\
\hline $\begin{array}{c}\text { CR Field } 1 \\
\text { DR 4-1 }\end{array}$ & $\begin{array}{l}\text { Piney } \\
\text { Creek }\end{array}$ & 5 & 5 & 5.6 & 2.80 & 354.5 & 730 & 2 & 14.568 & 4.05E-03 \\
\hline $\begin{array}{c}\text { CR Field } 1 \\
\text { DR 4-1 }\end{array}$ & $\begin{array}{l}\text { Piney } \\
\text { Creek }\end{array}$ & 8 & 6 & 8 & 2.67 & 506.4 & 730 & 3 & 13.874 & 3.85E-03 \\
\hline $\begin{array}{c}\text { CR Field } 1 \\
\text { DR 4-1 }\end{array}$ & $\begin{array}{l}\text { Piney } \\
\text { Creek }\end{array}$ & 10 & 7 & 4.4 & 2.20 & 278.5 & 730 & 2 & 11.446 & 3.18E-03 \\
\hline $\begin{array}{c}\text { CR Field } 1 \\
\text { DR 4-1 }\end{array}$ & $\begin{array}{l}\text { Piney } \\
\text { Creek }\end{array}$ & 13 & 9 & 1.9 & 0.63 & 120.3 & 730 & 3 & 3.295 & $9.15 E-04$ \\
\hline $\begin{array}{c}\text { CR Field } 1 \\
\text { DR 4-1 }\end{array}$ & $\begin{array}{l}\text { Piney } \\
\text { Creek }\end{array}$ & 16 & 10 & 4.5 & 1.50 & 284.9 & 730 & 3 & 7.804 & 2.17E-03 \\
\hline $\begin{array}{c}\text { CR Field } 1 \\
\text { DR 4-1 }\end{array}$ & $\begin{array}{l}\text { Piney } \\
\text { Creek }\end{array}$ & 22 & 11 & 10.8 & 1.80 & 683.6 & 730 & 6 & 9.365 & $2.60 \mathrm{E}-03$ \\
\hline $\begin{array}{c}\text { CR Field } 1 \\
\text { DR 4-1 }\end{array}$ & $\begin{array}{l}\text { Piney } \\
\text { Creek }\end{array}$ & 28 & 12 & 9.9 & 1.65 & 626.7 & 730 & 6 & 8.585 & $2.38 \mathrm{E}-03$ \\
\hline $\begin{array}{c}\text { CR Field } 1 \\
\text { DR 4-1 }\end{array}$ & $\begin{array}{l}\text { Piney } \\
\text { Creek }\end{array}$ & 34 & 13 & 8.2 & 1.37 & 519.1 & 730 & 6 & 7.110 & $1.98 \mathrm{E}-03$ \\
\hline $\begin{array}{c}\text { CR Field } 1 \\
\text { DR 4-1 }\end{array}$ & $\begin{array}{l}\text { Piney } \\
\text { Creek }\end{array}$ & 40 & 14 & 2 & 0.33 & 126.6 & 730 & 6 & 1.734 & 4.82E-04 \\
\hline $\begin{array}{c}\text { CR Field } 1 \\
\text { DR 4-1 }\end{array}$ & $\begin{array}{l}\text { Piney } \\
\text { Creek }\end{array}$ & 46 & 15 & 7.1 & 1.18 & 449.4 & 730 & 6 & 6.157 & 1.71E-03 \\
\hline $\begin{array}{c}\text { CR Field } 1 \\
\text { DR 4-1 }\end{array}$ & $\begin{array}{l}\text { Piney } \\
\text { Creek }\end{array}$ & 59 & 17 & 3.5 & 0.27 & 221.6 & 730 & 13 & 1.401 & 3.89E-04 \\
\hline $\begin{array}{c}\text { CR Field } 1 \\
\text { DR 4-1 }\end{array}$ & $\begin{array}{l}\text { Piney } \\
\text { Creek }\end{array}$ & 65 & 18 & 3.9 & 0.65 & 246.9 & 730 & 6 & 3.382 & 9.39E-04 \\
\hline $\begin{array}{c}\text { CR Field } 1 \\
\text { DR 4-1 }\end{array}$ & $\begin{array}{l}\text { Piney } \\
\text { Creek }\end{array}$ & 71 & 19 & 2 & 0.33 & 126.6 & 730 & 6 & 1.734 & $4.82 \mathrm{E}-04$ \\
\hline $\begin{array}{c}\text { CR Field } 1 \\
\text { DR 4-1 }\end{array}$ & $\begin{array}{l}\text { Piney } \\
\text { Creek }\end{array}$ & 77 & 20 & 9.5 & 1.58 & 601.4 & 730 & 6 & 8.238 & 2.29E-03 \\
\hline $\begin{array}{c}\text { CR Field } 1 \\
\text { DR 4-1 }\end{array}$ & $\begin{array}{l}\text { Piney } \\
\text { Creek }\end{array}$ & 84 & 21 & 15.9 & 2.27 & 1006.5 & 730 & 7 & 11.818 & $3.28 \mathrm{E}-03$ \\
\hline $\begin{array}{c}\text { CR Field } 1 \\
\text { DR 4-1 }\end{array}$ & $\begin{array}{l}\text { Piney } \\
\text { Creek }\end{array}$ & 86 & 22 & 1.5 & 0.75 & 95.0 & 730 & 2 & 3.902 & $1.08 \mathrm{E}-03$ \\
\hline $\begin{array}{c}\text { CR Field } 1 \\
\text { DR 4-1 }\end{array}$ & $\begin{array}{l}\text { Piney } \\
\text { Creek }\end{array}$ & 88 & 23 & 1.3 & 0.65 & 82.3 & 730 & 2 & 3.382 & 9.39E-04 \\
\hline $\begin{array}{c}\text { CR Field } 1 \\
\text { DR 4-1 }\end{array}$ & $\begin{array}{l}\text { Piney } \\
\text { Creek }\end{array}$ & 90 & 24 & 1.8 & 0.90 & 113.9 & 730 & 2 & 4.682 & $1.30 \mathrm{E}-03$ \\
\hline $\begin{array}{c}\text { CR Field } 1 \\
\text { DR 4-1 }\end{array}$ & $\begin{array}{l}\text { Piney } \\
\text { Creek }\end{array}$ & 92 & 25 & 2.2 & 1.10 & 139.3 & 730 & 2 & 5.723 & 1.59E-03 \\
\hline $\begin{array}{c}\text { CR Field } 1 \\
\text { DR 4-1 }\end{array}$ & $\begin{array}{l}\text { Piney } \\
\text { Creek }\end{array}$ & 94 & 26 & 2.4 & 1.20 & 151.9 & 730 & 2 & 6.243 & 1.73E-03 \\
\hline $\begin{array}{c}\text { CR Field } 1 \\
\text { DR 4-1 }\end{array}$ & $\begin{array}{l}\text { Piney } \\
\text { Creek }\end{array}$ & 96 & 27 & 2.3 & 1.15 & 145.6 & 730 & 2 & 5.983 & $1.66 \mathrm{E}-03$ \\
\hline \multirow[t]{2}{*}{$\begin{array}{c}\text { CR Field } 1 \\
\text { DR 4-1 }\end{array}$} & $\begin{array}{l}\text { Piney } \\
\text { Creek }\end{array}$ & 98 & 28 & 2.4 & 1.20 & 151.9 & 730 & 2 & 6.243 & 1.73E-03 \\
\hline & & & & & & & & $\begin{array}{r}\text { Final } \\
\text { Infiltration } \\
\text { Rate: }\end{array}$ & 6.16 & $1.71 \mathrm{E}-03$ \\
\hline & & & & & & & & & & \\
\hline \multicolumn{11}{|l|}{ 6/18/2003 } \\
\hline $\begin{array}{l}\text { 2nd Test- } \\
\text { Same } \\
\text { location }\end{array}$ & & & & & & & & & & \\
\hline
\end{tabular}




\begin{tabular}{|c|c|c|c|c|c|c|c|c|c|c|}
\hline Test ID & $\begin{array}{l}\text { Water } \\
\text { Used }\end{array}$ & $\begin{array}{l}\text { Time } \\
\text { min }\end{array}$ & Reading & $\begin{array}{c}\text { Flow } \\
\text { Reading, cm }\end{array}$ & $\begin{array}{l}\text { Flow, } \\
\mathrm{cm} / \mathrm{min}\end{array}$ & $\begin{array}{l}V_{\mathrm{IR}}, \\
\mathrm{cm}^{3}\end{array}$ & $\begin{array}{l}A_{\mathrm{IR}}, \\
\mathrm{cm}^{2}\end{array}$ & $t, \min$ & $V_{\mathrm{IR}}, \mathrm{cm} / \mathrm{h}$ & $V_{\mathrm{IR}}, \mathrm{cm} / \mathrm{s}$ \\
\hline $\begin{array}{c}\text { CR Field } 1 \\
\text { DR 3-2 }\end{array}$ & $\begin{array}{l}\text { Piney } \\
\text { Creek }\end{array}$ & 1 & 1 & 5.70 & 5.70 & 360.8 & 725 & 1 & 29.860 & $8.29 \mathrm{E}-03$ \\
\hline $\begin{array}{c}\text { CR Field } 1 \\
\text { DR 3-2 }\end{array}$ & $\begin{array}{l}\text { Piney } \\
\text { Creek }\end{array}$ & 2 & 2 & 5.7 & 5.70 & 360.8 & 725 & 1 & 29.860 & 8.29E-03 \\
\hline $\begin{array}{c}\text { CR Field } 1 \\
\text { DR 3-2 }\end{array}$ & $\begin{array}{l}\text { Piney } \\
\text { Creek }\end{array}$ & 3 & 3 & 5.6 & 5.60 & 354.5 & 725 & 1 & 29.336 & 8.15E-03 \\
\hline $\begin{array}{c}\text { CR Field } 1 \\
\text { DR 3-2 }\end{array}$ & $\begin{array}{l}\text { Piney } \\
\text { Creek }\end{array}$ & 5 & 4 & 11.7 & 5.85 & 740.6 & 725 & 2 & 30.646 & $8.51 \mathrm{E}-03$ \\
\hline $\begin{array}{c}\text { CR Field } 1 \\
\text { DR 3-2 }\end{array}$ & $\begin{array}{l}\text { Piney } \\
\text { Creek }\end{array}$ & 7 & 5 & 12.5 & 6.25 & 791.3 & 725 & 2 & 32.741 & $9.09 \mathrm{E}-03$ \\
\hline $\begin{array}{c}\text { CR Field } 1 \\
\text { DR 3-2 }\end{array}$ & $\begin{array}{l}\text { Piney } \\
\text { Creek }\end{array}$ & 8 & 6 & 6 & 6.00 & 379.8 & 725 & 1 & 31.432 & 8.73E-03 \\
\hline $\begin{array}{c}\text { CR Field } 1 \\
\text { DR 3-2 }\end{array}$ & $\begin{array}{l}\text { Piney } \\
\text { Creek }\end{array}$ & 10 & 7 & 7.6 & 3.80 & 481.1 & 725 & 2 & 19.907 & 5.53E-03 \\
\hline $\begin{array}{c}\text { CR Field } 1 \\
\text { DR 3-2 }\end{array}$ & $\begin{array}{l}\text { Piney } \\
\text { Creek }\end{array}$ & 11 & 8 & 5.7 & 5.70 & 360.8 & 725 & 1 & 29.860 & 8.29E-03 \\
\hline $\begin{array}{c}\text { CR Field } 1 \\
\text { DR 3-2 }\end{array}$ & $\begin{array}{l}\text { Piney } \\
\text { Creek }\end{array}$ & 12 & 9 & 5.3 & 5.30 & 335.5 & 725 & 1 & 27.765 & $7.71 \mathrm{E}-03$ \\
\hline $\begin{array}{c}\text { CR Field } 1 \\
\text { DR 3-2 }\end{array}$ & $\begin{array}{l}\text { Piney } \\
\text { Creek }\end{array}$ & 17 & 10 & 24.4 & 4.88 & 1544.5 & 725 & 5 & 25.564 & 7.10E-03 \\
\hline $\begin{array}{c}\text { CR Field } 1 \\
\text { DR 3-2 }\end{array}$ & $\begin{array}{l}\text { Piney } \\
\text { Creek }\end{array}$ & 18 & 11 & 5.1 & 5.10 & 322.8 & 725 & 1 & 26.717 & 7.42E-03 \\
\hline $\begin{array}{c}\text { CR Field } 1 \\
\text { DR 3-2 }\end{array}$ & $\begin{array}{l}\text { Piney } \\
\text { Creek }\end{array}$ & 20 & 12 & 5 & 2.50 & 316.5 & 725 & 2 & 13.097 & 3.64E-03 \\
\hline $\begin{array}{c}\text { CR Field } 1 \\
\text { DR 3-2 }\end{array}$ & $\begin{array}{l}\text { Piney } \\
\text { Creek }\end{array}$ & 22 & 13 & 9 & 4.50 & 569.7 & 725 & 2 & 23.574 & $6.55 \mathrm{E}-03$ \\
\hline $\begin{array}{c}\text { CR Field } 1 \\
\text { DR 3-2 }\end{array}$ & $\begin{array}{l}\text { Piney } \\
\text { Creek }\end{array}$ & 28 & 14 & 25.1 & 4.18 & 1588.8 & 725 & 6 & 21.915 & 6.09E-03 \\
\hline $\begin{array}{c}\text { CR Field } 1 \\
\text { DR 3-2 }\end{array}$ & $\begin{array}{l}\text { Piney } \\
\text { Creek }\end{array}$ & 31 & 15 & 12.9 & 4.30 & 816.6 & 725 & 3 & 22.526 & $6.26 \mathrm{E}-03$ \\
\hline $\begin{array}{c}\text { CR Field } 1 \\
\text { DR 3-2 }\end{array}$ & $\begin{array}{l}\text { Piney } \\
\text { Creek }\end{array}$ & 35 & 16 & 13.6 & 3.40 & 860.9 & 725 & 4 & 17.811 & 4.95E-03 \\
\hline $\begin{array}{c}\text { CR Field } 1 \\
\text { DR 3-2 }\end{array}$ & $\begin{array}{l}\text { Piney } \\
\text { Creek }\end{array}$ & 50 & 17 & 16 & 1.07 & 1012.8 & 725 & 15 & 5.588 & $1.55 \mathrm{E}-03$ \\
\hline $\begin{array}{c}\text { CR Field } 1 \\
\text { DR 3-2 }\end{array}$ & $\begin{array}{l}\text { Piney } \\
\text { Creek }\end{array}$ & 56 & 18 & 20.4 & 3.40 & 1291.3 & 725 & 6 & 17.811 & 4.95E-03 \\
\hline $\begin{array}{c}\text { CR Field } 1 \\
\text { DR 3-2 }\end{array}$ & $\begin{array}{l}\text { Piney } \\
\text { Creek }\end{array}$ & 59 & 19 & 6 & 2.00 & 379.8 & 725 & 3 & 10.477 & 2.91E-03 \\
\hline $\begin{array}{c}\text { CR Field } 1 \\
\text { DR 3-2 }\end{array}$ & $\begin{array}{l}\text { Piney } \\
\text { Creek }\end{array}$ & 63 & 20 & 14.5 & 3.63 & 917.9 & 725 & 4 & 18.990 & $5.28 \mathrm{E}-03$ \\
\hline $\begin{array}{c}\text { CR Field } 1 \\
\text { DR 3-2 }\end{array}$ & $\begin{array}{l}\text { Piney } \\
\text { Creek }\end{array}$ & 69 & 21 & 15 & 2.50 & 949.5 & 725 & 6 & 13.097 & $3.64 \mathrm{E}-03$ \\
\hline $\begin{array}{c}\text { CR Field } 1 \\
\text { DR 3-2 }\end{array}$ & $\begin{array}{l}\text { Piney } \\
\text { Creek }\end{array}$ & 74 & 22 & 17.6 & 3.52 & 1114.1 & 725 & 5 & 18.440 & 5.12E-03 \\
\hline $\begin{array}{c}\text { CR Field } 1 \\
\text { DR 3-2 }\end{array}$ & $\begin{array}{l}\text { Piney } \\
\text { Creek }\end{array}$ & 77 & 23 & 7 & 2.33 & 443.1 & 725 & 3 & 12.223 & $3.40 \mathrm{E}-03$ \\
\hline $\begin{array}{c}\text { CR Field } 1 \\
\text { DR 3-2 }\end{array}$ & $\begin{array}{l}\text { Piney } \\
\text { Creek }\end{array}$ & 82 & 24 & 12.8 & 2.56 & 810.2 & 725 & 5 & 13.411 & 3.73E-03 \\
\hline $\begin{array}{c}\text { CR Field } 1 \\
\text { DR 3-2 }\end{array}$ & $\begin{array}{l}\text { Piney } \\
\text { Creek }\end{array}$ & 87 & 25 & 16.5 & 3.30 & 1044.5 & 725 & 5 & 17.287 & $4.80 \mathrm{E}-03$ \\
\hline $\begin{array}{c}\text { CR Field } 1 \\
\text { DR 3-2 }\end{array}$ & $\begin{array}{l}\text { Piney } \\
\text { Creek }\end{array}$ & 91 & 26 & 16.5 & 4.13 & 1044.5 & 725 & 4 & 21.609 & $6.00 \mathrm{E}-03$ \\
\hline $\begin{array}{c}\text { CR Field } 1 \\
\text { DR 3-2 }\end{array}$ & $\begin{array}{l}\text { Piney } \\
\text { Creek }\end{array}$ & 93 & 27 & 5.2 & 2.60 & 329.2 & 725 & 2 & 13.620 & $3.78 \mathrm{E}-03$ \\
\hline CR Field 1 & Piney & 100 & 28 & 24.1 & 3.44 & 1525.5 & 725 & 7 & 18.036 & $5.01 \mathrm{E}-03$ \\
\hline
\end{tabular}




\begin{tabular}{|c|c|c|c|c|c|c|c|c|c|c|}
\hline DR 3-2 & Creek & & & & & & & & & \\
\hline $\begin{array}{c}\text { CR Field } 1 \\
\text { DR 3-2 }\end{array}$ & $\begin{array}{l}\text { Piney } \\
\text { Creek }\end{array}$ & 104 & 29 & 10.6 & 2.65 & 671.0 & 725 & 4 & 13.882 & $3.86 \mathrm{E}-03$ \\
\hline & & & & & & & & $\begin{array}{r}\text { Final } \\
\text { Infiltration } \\
\text { Rate: }\end{array}$ & 14.74 & 4.10E-03 \\
\hline & & & & & & & & & & \\
\hline & & & & & & & & & & \\
\hline \multicolumn{11}{|l|}{ 6/18/2003 } \\
\hline \multicolumn{11}{|l|}{\begin{tabular}{|l} 
2nd Test- \\
Same \\
location \\
\end{tabular}} \\
\hline Test ID & $\begin{array}{l}\text { Water } \\
\text { Used }\end{array}$ & $\begin{array}{l}\text { Time } \\
\text { min }\end{array}$ & Reading & $\begin{array}{c}\text { Flow } \\
\text { Reading, cm }\end{array}$ & $\begin{array}{l}\text { Flow, } \\
\mathrm{cm} / \mathrm{min}\end{array}$ & $\begin{array}{r}\begin{array}{l}V_{\mathrm{IR}}, \\
\mathrm{cm}^{3}\end{array} \\
\mathrm{c}^{2}\end{array}$ & $\begin{array}{l}A_{\mathrm{IR}}, \\
\mathrm{cm}^{2}\end{array}$ & $t, \min$ & $V_{\mathrm{IR}}, \mathrm{cm} / \mathrm{h}$ & $V_{\mathrm{IR}}, \mathrm{cm} / \mathrm{s}$ \\
\hline $\begin{array}{c}\text { CR Field } 1 \\
\text { DR 4-2 }\end{array}$ & $\begin{array}{c}\text { CBM- } \\
\text { CR }\end{array}$ & 3 & 1 & 7.20 & 2.40 & 455.8 & 725 & 3 & 12.573 & $3.49 \mathrm{E}-03$ \\
\hline $\begin{array}{c}\text { CR Field } 1 \\
\text { DR 4-2 }\end{array}$ & $\begin{array}{c}\text { CBM- } \\
\text { CR }\end{array}$ & 5 & 2 & 3.7 & 1.85 & 234.2 & 725 & 2 & 9.691 & $2.69 \mathrm{E}-03$ \\
\hline $\begin{array}{c}\text { CR Field } 1 \\
\text { DR 4-2 }\end{array}$ & $\begin{array}{c}\text { CBM- } \\
\text { CR }\end{array}$ & 15 & 3 & 9.4 & 0.94 & 595.0 & 725 & 10 & 4.924 & 1.37E-03 \\
\hline $\begin{array}{c}\text { CR Field } 1 \\
\text { DR 4-2 }\end{array}$ & $\begin{array}{c}\text { CBM- } \\
\text { CR }\end{array}$ & 20 & 4 & 2.3 & 0.46 & 145.6 & 725 & 5 & 2.410 & 6.69E-04 \\
\hline $\begin{array}{c}\text { CR Field } 1 \\
\text { DR 4-2 }\end{array}$ & $\begin{array}{c}\text { CBM- } \\
\text { CR }\end{array}$ & 28 & 5 & 1.4 & 0.18 & 88.6 & 725 & 8 & 0.917 & $2.55 \mathrm{E}-04$ \\
\hline $\begin{array}{c}\text { CR Field } 1 \\
\text { DR 4-2 }\end{array}$ & $\begin{array}{c}\text { CBM- } \\
\text { CR }\end{array}$ & 36 & 6 & 4.4 & 0.55 & 278.5 & 725 & 8 & 2.881 & $8.00 \mathrm{E}-04$ \\
\hline $\begin{array}{c}\text { CR Field } 1 \\
\text { DR 4-2 }\end{array}$ & $\begin{array}{c}\text { CBM- } \\
\text { CR }\end{array}$ & 44 & 7 & 13.1 & 1.64 & 829.2 & 725 & 8 & 8.578 & 2.38E-03 \\
\hline $\begin{array}{c}\text { CR Field } 1 \\
\text { DR 4-2 }\end{array}$ & $\begin{array}{c}\text { CBM- } \\
\text { CR }\end{array}$ & 49 & 8 & 3.9 & 0.78 & 246.9 & 725 & 5 & 4.086 & $1.14 \mathrm{E}-03$ \\
\hline $\begin{array}{c}\text { CR Field } 1 \\
\text { DR 4-2 }\end{array}$ & $\begin{array}{c}\text { CBM- } \\
\text { CR }\end{array}$ & 57 & 10 & 2.9 & 0.36 & 183.6 & 725 & 8 & 1.899 & $5.28 \mathrm{E}-04$ \\
\hline $\begin{array}{c}\text { CR Field } 1 \\
\text { DR 4-2 }\end{array}$ & $\begin{array}{c}\text { CBM- } \\
\text { CR }\end{array}$ & 64 & 11 & 3.2 & 0.46 & 202.6 & 725 & 7 & 2.395 & 6.65E-04 \\
\hline $\begin{array}{c}\text { CR Field } 1 \\
\text { DR 4-2 }\end{array}$ & $\begin{array}{c}\text { CBM- } \\
\text { CR }\end{array}$ & 72 & 12 & 9.6 & 1.20 & 607.7 & 725 & 8 & 6.286 & $1.75 \mathrm{E}-03$ \\
\hline $\begin{array}{c}\text { CR Field } 1 \\
\text { DR 4-2 }\end{array}$ & $\begin{array}{c}\text { CBM- } \\
\text { CR }\end{array}$ & 84 & 13 & 10.9 & 0.91 & 690.0 & 725 & 12 & 4.758 & $1.32 \mathrm{E}-03$ \\
\hline $\begin{array}{c}\text { CR Field } 1 \\
\text { DR 4-2 }\end{array}$ & $\begin{array}{c}\text { CBM- } \\
\text { CR }\end{array}$ & 94 & 14 & 8 & 0.80 & 506.4 & 725 & 10 & 4.191 & $1.16 \mathrm{E}-03$ \\
\hline \multirow[t]{2}{*}{$\begin{array}{c}\text { CR Field } 1 \\
\text { DR 4-2 }\end{array}$} & $\begin{array}{c}\text { CBM- } \\
\text { CR }\end{array}$ & 99 & 15 & 5.8 & 1.16 & 367.1 & 725 & 5 & 6.077 & $1.69 \mathrm{E}-03$ \\
\hline & & & & & & & & $\begin{array}{r}\text { Final } \\
\text { Infiltration } \\
\text { Rate: }\end{array}$ & 5.33 & $1.48 \mathrm{E}-03$ \\
\hline & & & & & & & & & & \\
\hline & & & & & & & & & & \\
\hline \multicolumn{11}{|l|}{ 6/18/2003 } \\
\hline \multicolumn{11}{|l|}{ First Test } \\
\hline Test ID & $\begin{array}{l}\text { Water } \\
\text { Used }\end{array}$ & $\begin{array}{l}\text { Time } \\
\text { min }\end{array}$ & Reading & $\begin{array}{c}\text { Flow } \\
\text { Reading, cm }\end{array}$ & $\begin{array}{l}\text { Flow, } \\
\mathrm{cm} / \mathrm{min}\end{array}$ & $\begin{array}{l}\begin{array}{l}V_{\text {IR }}, \\
\mathrm{cm}^{3}\end{array}\end{array}$ & $\begin{array}{l}A_{\mathrm{IR}}, \\
\mathrm{cm}^{2}\end{array}$ & $t, \min$ & $V_{\mathrm{IR}}, \mathrm{cm} / \mathrm{h}$ & $V_{\mathrm{IR}}, \mathrm{cm} / \mathrm{s}$ \\
\hline $\begin{array}{c}\text { CR Field } 1 \\
\text { DR 5-1 }\end{array}$ & $\begin{array}{l}\text { Piney } \\
\text { Creek }\end{array}$ & 2 & 1 & 7.60 & 7.60 & 481.1 & 730 & 1 & 39.541 & $1.10 \mathrm{E}-02$ \\
\hline
\end{tabular}




\begin{tabular}{|c|c|c|c|c|c|c|c|c|c|c|}
\hline $\begin{array}{c}\text { CR Field } 1 \\
\text { DR 5-1 }\end{array}$ & $\begin{array}{l}\text { Piney } \\
\text { Creek }\end{array}$ & 2.5 & 2 & 5.2 & 5.20 & 329.2 & 730 & 1 & 27.054 & 7.52E-03 \\
\hline $\begin{array}{c}\text { CR Field } 1 \\
\text { DR 5-1 }\end{array}$ & $\begin{array}{l}\text { Piney } \\
\text { Creek }\end{array}$ & 3 & 3 & 2.1 & 4.20 & 132.9 & 730 & 1 & 21.852 & 6.07E-03 \\
\hline $\begin{array}{c}\text { CR Field } 1 \\
\text { DR 5-1 }\end{array}$ & $\begin{array}{l}\text { Piney } \\
\text { Creek }\end{array}$ & 4 & 4 & 3.1 & 3.10 & 196.2 & 730 & 1 & 16.128 & $4.48 \mathrm{E}-03$ \\
\hline $\begin{array}{c}\text { CR Field } 1 \\
\text { DR 5-1 }\end{array}$ & $\begin{array}{l}\text { Piney } \\
\text { Creek }\end{array}$ & 5 & 5 & 0.2 & 0.20 & 12.7 & 730 & 1 & 1.041 & 2.89E-04 \\
\hline $\begin{array}{c}\text { CR Field } 1 \\
\text { DR 5-1 }\end{array}$ & $\begin{array}{l}\text { Piney } \\
\text { Creek }\end{array}$ & 8 & 6 & 4.7 & 1.57 & 297.5 & 730 & 3 & 8.151 & $2.26 \mathrm{E}-03$ \\
\hline $\begin{array}{c}\text { CR Field } 1 \\
\text { DR 5-1 }\end{array}$ & $\begin{array}{l}\text { Piney } \\
\text { Creek }\end{array}$ & 13 & 7 & 13.4 & 2.68 & 848.2 & 730 & 5 & 13.943 & 3.87E-03 \\
\hline $\begin{array}{c}\text { CR Field } 1 \\
\text { DR 5-1 }\end{array}$ & $\begin{array}{l}\text { Piney } \\
\text { Creek }\end{array}$ & 21 & 8 & 8.8 & 1.10 & 557.0 & 730 & 8 & 5.723 & $1.59 \mathrm{E}-03$ \\
\hline $\begin{array}{c}\text { CR Field } 1 \\
\text { DR 5-1 }\end{array}$ & $\begin{array}{l}\text { Piney } \\
\text { Creek }\end{array}$ & 30 & 12 & 1 & 0.11 & 63.3 & 730 & 9 & 0.578 & $1.61 \mathrm{E}-04$ \\
\hline $\begin{array}{c}\text { CR Field } 1 \\
\text { DR 5-1 }\end{array}$ & $\begin{array}{l}\text { Piney } \\
\text { Creek }\end{array}$ & 31 & 13 & 0.3 & 0.30 & 19.0 & 730 & 1 & 1.561 & 4.34E-04 \\
\hline $\begin{array}{c}\text { CR Field } 1 \\
\text { DR 5-1 }\end{array}$ & $\begin{array}{l}\text { Piney } \\
\text { Creek }\end{array}$ & 38 & 14 & 18.8 & 2.69 & 1190.0 & 730 & 7 & 13.973 & $3.88 \mathrm{E}-03$ \\
\hline $\begin{array}{c}\text { CR Field } 1 \\
\text { DR 5-1 }\end{array}$ & $\begin{array}{l}\text { Piney } \\
\text { Creek }\end{array}$ & 45 & 15 & 2.9 & 0.41 & 183.6 & 730 & 7 & 2.155 & 5.99E-04 \\
\hline $\begin{array}{c}\text { CR Field } 1 \\
\text { DR 5-1 }\end{array}$ & $\begin{array}{l}\text { Piney } \\
\text { Creek }\end{array}$ & 60 & 16 & 10.5 & 0.70 & 664.7 & 730 & 15 & 3.642 & $1.01 \mathrm{E}-03$ \\
\hline $\begin{array}{c}\text { CR Field } 1 \\
\text { DR 5-1 }\end{array}$ & $\begin{array}{l}\text { Piney } \\
\text { Creek }\end{array}$ & 72 & 17 & 15.7 & 1.31 & 993.8 & 730 & 12 & 6.807 & 1.89E-03 \\
\hline $\begin{array}{c}\text { CR Field } 1 \\
\text { DR 5-1 }\end{array}$ & $\begin{array}{l}\text { Piney } \\
\text { Creek }\end{array}$ & 73 & 18 & 1.9 & 1.90 & 120.3 & 730 & 1 & 9.885 & 2.75E-03 \\
\hline $\begin{array}{c}\text { CR Field } 1 \\
\text { DR 5-1 }\end{array}$ & $\begin{array}{l}\text { Piney } \\
\text { Creek }\end{array}$ & 75 & 20 & 0.7 & 0.35 & 44.3 & 730 & 2 & 1.821 & 5.06E-04 \\
\hline $\begin{array}{c}\text { CR Field } 1 \\
\text { DR 5-1 }\end{array}$ & $\begin{array}{l}\text { Piney } \\
\text { Creek }\end{array}$ & 76 & 21 & 0.5 & 0.50 & 31.7 & 730 & 1 & 2.601 & 7.23E-04 \\
\hline $\begin{array}{c}\text { CR Field } 1 \\
\text { DR 5-1 }\end{array}$ & $\begin{array}{l}\text { Piney } \\
\text { Creek }\end{array}$ & 82 & 22 & 5.5 & 0.92 & 348.2 & 730 & 6 & 4.769 & $1.32 \mathrm{E}-03$ \\
\hline $\begin{array}{c}\text { CR Field } 1 \\
\text { DR 5-1 }\end{array}$ & $\begin{array}{l}\text { Piney } \\
\text { Creek }\end{array}$ & 89 & 23 & 3.4 & 0.49 & 215.2 & 730 & 7 & 2.527 & 7.02E-04 \\
\hline $\begin{array}{c}\text { CR Field } 1 \\
\text { DR 5-1 }\end{array}$ & $\begin{array}{l}\text { Piney } \\
\text { Creek }\end{array}$ & 96 & 24 & 4.5 & 0.64 & 284.9 & 730 & 7 & 3.345 & $9.29 \mathrm{E}-04$ \\
\hline $\begin{array}{c}\text { CR Field } 1 \\
\text { DR 5-1 }\end{array}$ & $\begin{array}{l}\text { Piney } \\
\text { Creek }\end{array}$ & 107 & 25 & 13 & 1.18 & 822.9 & 730 & 11 & 6.149 & $1.71 \mathrm{E}-03$ \\
\hline $\begin{array}{c}\text { CR Field } 1 \\
\text { DR 5-1 }\end{array}$ & $\begin{array}{l}\text { Piney } \\
\text { Creek }\end{array}$ & 112 & 26 & 3.2 & 0.64 & 202.6 & 730 & 5 & 3.330 & $9.25 \mathrm{E}-04$ \\
\hline $\begin{array}{c}\text { CR Field } 1 \\
\text { DR 5-1 }\end{array}$ & $\begin{array}{l}\text { Piney } \\
\text { Creek }\end{array}$ & 118 & 27 & 4.8 & 0.80 & 303.8 & 730 & 6 & 4.162 & $1.16 \mathrm{E}-03$ \\
\hline $\begin{array}{c}\text { CR Field } 1 \\
\text { DR 5-1 }\end{array}$ & $\begin{array}{l}\text { Piney } \\
\text { Creek }\end{array}$ & 124 & 28 & 4.8 & 0.80 & 303.8 & 730 & 6 & 4.162 & 1.16E-03 \\
\hline & & & & & & & & $\begin{array}{r}\text { Final } \\
\text { Infiltration } \\
\text { Rate: }\end{array}$ & 4.23 & 1.17E-03 \\
\hline & & & & & & & & & & \\
\hline & & & & & & & & & & \\
\hline \multirow{2}{*}{\multicolumn{11}{|c|}{ 6/18/2003 }} \\
\hline & & & & & & & & & & \\
\hline \multicolumn{11}{|l|}{ First Test } \\
\hline Test ID & $\begin{array}{l}\text { Water } \\
\text { Used }\end{array}$ & $\begin{array}{l}\text { Time } \\
\text { min }\end{array}$ & Reading & $\begin{array}{c}\text { Flow } \\
\text { Reading, cm }\end{array}$ & $\begin{array}{l}\text { Flow, } \\
\mathrm{cm} / \mathrm{min}\end{array}$ & $\begin{array}{r}\begin{array}{l}V_{\mathrm{IR}}, \\
\mathrm{cm}^{3}\end{array} \\
\end{array}$ & $\begin{array}{l}A_{\mathrm{IR}} \\
\mathrm{cm}^{2}\end{array}$ & $t, \min$ & $V_{\mathrm{IR}}, \mathrm{cm} / \mathrm{h}$ & $V_{\mathrm{IR}}, \mathrm{cm} / \mathrm{s}$ \\
\hline
\end{tabular}




\begin{tabular}{|c|c|c|c|c|c|c|c|c|c|c|}
\hline $\begin{array}{c}\text { CR Field } 1 \\
\text { DR 6-1 }\end{array}$ & $\begin{array}{l}\text { Piney } \\
\text { Creek }\end{array}$ & 1 & 1 & 8.90 & 8.90 & 563.4 & 730 & 1 & 46.304 & $1.29 \mathrm{E}-02$ \\
\hline $\begin{array}{c}\text { CR Field } 1 \\
\text { DR 6-1 }\end{array}$ & $\begin{array}{l}\text { Piney } \\
\text { Creek }\end{array}$ & 1.5 & 2 & 9.7 & 19.40 & 614.0 & 730 & 1 & 100.933 & $2.80 \mathrm{E}-02$ \\
\hline $\begin{array}{c}\text { CR Field } 1 \\
\text { DR 6-1 }\end{array}$ & $\begin{array}{l}\text { Piney } \\
\text { Creek }\end{array}$ & 3 & 3 & 10.3 & 6.87 & 652.0 & 730 & 2 & 35.725 & 9.92E-03 \\
\hline $\begin{array}{c}\text { CR Field } 1 \\
\text { DR 6-1 }\end{array}$ & $\begin{array}{l}\text { Piney } \\
\text { Creek }\end{array}$ & 5 & 4 & 6.1 & 3.05 & 386.1 & 730 & 2 & 15.868 & 4.41E-03 \\
\hline $\begin{array}{c}\text { CR Field } 1 \\
\text { DR 6-1 }\end{array}$ & $\begin{array}{l}\text { Piney } \\
\text { Creek }\end{array}$ & 6 & 5 & 4.9 & 4.90 & 310.2 & 730 & 1 & 25.493 & 7.08E-03 \\
\hline $\begin{array}{c}\text { CR Field } 1 \\
\text { DR 6-1 }\end{array}$ & $\begin{array}{l}\text { Piney } \\
\text { Creek }\end{array}$ & 8 & 6 & 10.7 & 5.35 & 677.3 & 730 & 2 & 27.835 & 7.73E-03 \\
\hline $\begin{array}{c}\text { CR Field } 1 \\
\text { DR 6-1 }\end{array}$ & $\begin{array}{l}\text { Piney } \\
\text { Creek }\end{array}$ & 13 & 7 & 8.5 & 1.70 & 538.1 & 730 & 5 & 8.845 & $2.46 \mathrm{E}-03$ \\
\hline $\begin{array}{c}\text { CR Field } 1 \\
\text { DR 6-1 }\end{array}$ & $\begin{array}{l}\text { Piney } \\
\text { Creek }\end{array}$ & 18 & 8 & 16.6 & 3.32 & 1050.8 & 730 & 5 & 17.273 & $4.80 \mathrm{E}-03$ \\
\hline $\begin{array}{c}\text { CR Field } 1 \\
\text { DR 6-1 }\end{array}$ & $\begin{array}{l}\text { Piney } \\
\text { Creek }\end{array}$ & 23 & 9 & 6.9 & 1.38 & 436.8 & 730 & 5 & 7.180 & $1.99 \mathrm{E}-03$ \\
\hline $\begin{array}{c}\text { CR Field } 1 \\
\text { DR 6-1 }\end{array}$ & $\begin{array}{l}\text { Piney } \\
\text { Creek }\end{array}$ & 30 & 10 & 6.6 & 0.94 & 417.8 & 730 & 7 & 4.905 & $1.36 \mathrm{E}-03$ \\
\hline $\begin{array}{c}\text { CR Field } 1 \\
\text { DR 6-1 }\end{array}$ & $\begin{array}{l}\text { Piney } \\
\text { Creek }\end{array}$ & 32 & 12 & 1.5 & 0.75 & 95.0 & 730 & 2 & 3.902 & $1.08 \mathrm{E}-03$ \\
\hline $\begin{array}{c}\text { CR Field } 1 \\
\text { DR 6-1 }\end{array}$ & $\begin{array}{l}\text { Piney } \\
\text { Creek }\end{array}$ & 33 & 13 & 2.1 & 2.10 & 132.9 & 730 & 1 & 10.926 & 3.03E-03 \\
\hline $\begin{array}{c}\text { CR Field } 1 \\
\text { DR 6-1 }\end{array}$ & $\begin{array}{l}\text { Piney } \\
\text { Creek }\end{array}$ & 41 & 14 & 17 & 2.13 & 1076.1 & 730 & 8 & 11.056 & 3.07E-03 \\
\hline $\begin{array}{c}\text { CR Field } 1 \\
\text { DR 6-1 }\end{array}$ & $\begin{array}{l}\text { Piney } \\
\text { Creek }\end{array}$ & 52 & 15 & 9.1 & 0.83 & 576.0 & 730 & 11 & 4.304 & $1.20 \mathrm{E}-03$ \\
\hline $\begin{array}{c}\text { CR Field } 1 \\
\text { DR 6-1 }\end{array}$ & $\begin{array}{l}\text { Piney } \\
\text { Creek }\end{array}$ & 62 & 16 & 6.1 & 0.61 & 386.1 & 730 & 10 & 3.174 & 8.82E-04 \\
\hline $\begin{array}{c}\text { CR Field } 1 \\
\text { DR 6-1 }\end{array}$ & $\begin{array}{l}\text { Piney } \\
\text { Creek }\end{array}$ & 69 & 17 & 13.2 & 1.89 & 835.6 & 730 & 7 & 9.811 & 2.73E-03 \\
\hline $\begin{array}{c}\text { CR Field } 1 \\
\text { DR 6-1 }\end{array}$ & $\begin{array}{l}\text { Piney } \\
\text { Creek }\end{array}$ & 70 & 18 & 2.7 & 2.70 & 170.9 & 730 & 1 & 14.047 & $3.90 \mathrm{E}-03$ \\
\hline $\begin{array}{c}\text { CR Field } 1 \\
\text { DR 6-1 }\end{array}$ & $\begin{array}{l}\text { Piney } \\
\text { Creek }\end{array}$ & 72 & 19 & 1 & 0.50 & 63.3 & 730 & 2 & 2.601 & 7.23E-04 \\
\hline $\begin{array}{c}\text { CR Field } 1 \\
\text { DR 6-1 }\end{array}$ & $\begin{array}{l}\text { Piney } \\
\text { Creek }\end{array}$ & 73 & 20 & 1.4 & 1.40 & 88.6 & 730 & 1 & 7.284 & $2.02 \mathrm{E}-03$ \\
\hline $\begin{array}{c}\text { CR Field } 1 \\
\text { DR 6-1 }\end{array}$ & $\begin{array}{l}\text { Piney } \\
\text { Creek }\end{array}$ & 78 & 21 & 6 & 1.20 & 379.8 & 730 & 5 & 6.243 & $1.73 \mathrm{E}-03$ \\
\hline $\begin{array}{c}\text { CR Field } 1 \\
\text { DR 6-1 }\end{array}$ & $\begin{array}{l}\text { Piney } \\
\text { Creek }\end{array}$ & 87 & 22 & 11.5 & 1.28 & 728.0 & 730 & 9 & 6.648 & $1.85 \mathrm{E}-03$ \\
\hline $\begin{array}{c}\text { CR Field } 1 \\
\text { DR 6-1 }\end{array}$ & $\begin{array}{l}\text { Piney } \\
\text { Creek }\end{array}$ & 93 & 23 & 8.5 & 1.42 & 538.1 & 730 & 6 & 7.371 & $2.05 \mathrm{E}-03$ \\
\hline \multirow[t]{2}{*}{$\begin{array}{c}\text { CR Field } 1 \\
\text { DR 6-1 }\end{array}$} & $\begin{array}{l}\text { Piney } \\
\text { Creek }\end{array}$ & 99 & 24 & 8.1 & 1.35 & 512.7 & 730 & 6 & 7.024 & $1.95 \mathrm{E}-03$ \\
\hline & & & & & & & & $\begin{array}{r}\text { Final } \\
\text { Infiltration } \\
\text { Rate: }\end{array}$ & 6.82 & 1.89E-03 \\
\hline & & & & & & & & & & \\
\hline & & & & & & & & & & \\
\hline \multirow{2}{*}{\multicolumn{11}{|c|}{ 6/19/2003 }} \\
\hline & & & & & & & & & & \\
\hline $\begin{array}{l}\text { 2nd Test- } \\
\text { Same } \\
\text { location }\end{array}$ & & & & & & & & & & \\
\hline
\end{tabular}




\begin{tabular}{|c|c|c|c|c|c|c|c|c|c|c|}
\hline Test ID & $\begin{array}{l}\text { Water } \\
\text { Used }\end{array}$ & $\begin{array}{l}\text { Time } \\
\text { min }\end{array}$ & Reading & $\begin{array}{c}\text { Flow } \\
\text { Reading, cm }\end{array}$ & $\begin{array}{l}\text { Flow, } \\
\text { cm/min }\end{array}$ & $\begin{array}{r}\begin{array}{l}V_{\mathrm{IR}}, \\
\mathrm{cm}^{3}\end{array} \\
\text {, }\end{array}$ & $\begin{array}{l}A_{\mathrm{IR}}, \\
\mathrm{cm}^{2}\end{array}$ & $t, \min$ & $V_{\mathrm{IR}}, \mathrm{cm} / \mathrm{h}$ & $V_{\mathrm{IR}}, \mathrm{cm} / \mathrm{s}$ \\
\hline $\begin{array}{c}\text { CR Field } 1 \\
\text { DR 5-2 }\end{array}$ & $\begin{array}{l}\text { Piney } \\
\text { Creek }\end{array}$ & 1 & 1 & 0.7 & 0.70 & 44.3 & 725 & 1 & 3.667 & $1.02 \mathrm{E}-03$ \\
\hline $\begin{array}{c}\text { CR Field } 1 \\
\text { DR 5-2 }\end{array}$ & $\begin{array}{l}\text { Piney } \\
\text { Creek }\end{array}$ & 2 & 2 & 0.20 & 0.20 & 12.7 & 725 & 1 & 1.048 & 2.91E-04 \\
\hline $\begin{array}{c}\text { CR Field } 1 \\
\text { DR 5-2 }\end{array}$ & $\begin{array}{l}\text { Piney } \\
\text { Creek }\end{array}$ & 4 & 3 & 0.4 & 0.20 & 25.3 & 725 & 2 & 1.048 & $2.91 \mathrm{E}-04$ \\
\hline $\begin{array}{c}\text { CR Field } 1 \\
\text { DR 5-2 }\end{array}$ & $\begin{array}{l}\text { Piney } \\
\text { Creek }\end{array}$ & 6 & 4 & 0.9 & 0.45 & 57.0 & 725 & 2 & 2.357 & $6.55 \mathrm{E}-04$ \\
\hline $\begin{array}{c}\text { CR Field } 1 \\
\text { DR 5-2 }\end{array}$ & $\begin{array}{l}\text { Piney } \\
\text { Creek }\end{array}$ & 10 & 5 & 4.2 & 1.05 & 265.9 & 725 & 4 & 5.501 & $1.53 \mathrm{E}-03$ \\
\hline $\begin{array}{c}\text { CR Field } 1 \\
\text { DR 5-2 }\end{array}$ & $\begin{array}{l}\text { Piney } \\
\text { Creek }\end{array}$ & 16 & 6 & 6.1 & 1.02 & 386.1 & 725 & 6 & 5.326 & $1.48 \mathrm{E}-03$ \\
\hline $\begin{array}{c}\text { CR Field } 1 \\
\text { DR 5-2 }\end{array}$ & $\begin{array}{l}\text { Piney } \\
\text { Creek }\end{array}$ & 23 & 7 & 4.8 & 0.69 & 303.8 & 725 & 7 & 3.592 & 9.98E-04 \\
\hline $\begin{array}{c}\text { CR Field } 1 \\
\text { DR 5-2 }\end{array}$ & $\begin{array}{l}\text { Piney } \\
\text { Creek }\end{array}$ & 33 & 8 & 5.2 & 0.52 & 329.2 & 725 & 10 & 2.724 & 7.57E-04 \\
\hline $\begin{array}{l}\text { CR Field } 1 \\
\text { DR 5-2 }\end{array}$ & $\begin{array}{l}\text { Piney } \\
\text { Creek }\end{array}$ & 46 & 9 & 11.7 & 0.90 & 740.6 & 725 & 13 & 4.715 & $1.31 \mathrm{E}-03$ \\
\hline $\begin{array}{c}\text { CR Field } 1 \\
\text { DR 5-2 }\end{array}$ & $\begin{array}{l}\text { Piney } \\
\text { Creek }\end{array}$ & 54 & 10 & 9 & 1.13 & 569.7 & 725 & 8 & 5.893 & $1.64 \mathrm{E}-03$ \\
\hline $\begin{array}{l}\text { CR Field } 1 \\
\text { DR 5-2 }\end{array}$ & $\begin{array}{l}\text { Piney } \\
\text { Creek }\end{array}$ & 56 & 12 & 0.3 & 0.15 & 19.0 & 725 & 2 & 0.786 & $2.18 \mathrm{E}-04$ \\
\hline $\begin{array}{c}\text { CR Field } 1 \\
\text { DR 5-2 }\end{array}$ & $\begin{array}{l}\text { Piney } \\
\text { Creek }\end{array}$ & 57 & 13 & 0.3 & 0.30 & 19.0 & 725 & 1 & 1.572 & 4.37E-04 \\
\hline $\begin{array}{c}\text { CR Field } 1 \\
\text { DR 5-2 }\end{array}$ & $\begin{array}{l}\text { Piney } \\
\text { Creek }\end{array}$ & 63 & 14 & 1.3 & 0.22 & 82.3 & 725 & 6 & 1.135 & 3.15E-04 \\
\hline $\begin{array}{c}\text { CR Field } 1 \\
\text { DR 5-2 }\end{array}$ & $\begin{array}{l}\text { Piney } \\
\text { Creek }\end{array}$ & 70 & 15 & 8.7 & 1.24 & 550.7 & 725 & 7 & 6.511 & $1.81 \mathrm{E}-03$ \\
\hline $\begin{array}{c}\text { CR Field } 1 \\
\text { DR 5-2 }\end{array}$ & $\begin{array}{l}\text { Piney } \\
\text { Creek }\end{array}$ & 76 & 16 & 4.4 & 0.73 & 278.5 & 725 & 6 & 3.842 & 1.07E-03 \\
\hline $\begin{array}{l}\text { CR Field } 1 \\
\text { DR 5-2 }\end{array}$ & $\begin{array}{l}\text { Piney } \\
\text { Creek }\end{array}$ & 80 & 17 & 2.9 & 0.73 & 183.6 & 725 & 4 & 3.798 & $1.06 \mathrm{E}-03$ \\
\hline $\begin{array}{l}\text { CR Field } 1 \\
\text { DR 5-2 }\end{array}$ & $\begin{array}{l}\text { Piney } \\
\text { Creek }\end{array}$ & 85 & 18 & 3.7 & 0.74 & 234.2 & 725 & 5 & 3.877 & $1.08 \mathrm{E}-03$ \\
\hline & & & & & & & & $\begin{array}{r}\text { Final } \\
\text { Infiltration } \\
\text { Rate: }\end{array}$ & 3.84 & $1.07 \mathrm{E}-03$ \\
\hline & & & & & & & & & & \\
\hline & & & & & & & & & & \\
\hline \multicolumn{11}{|l|}{$6 / 19 / 2003$} \\
\hline \multicolumn{11}{|l|}{$\begin{array}{l}\text { 2nd Test- } \\
\text { Same } \\
\text { location }\end{array}$} \\
\hline Test ID & $\begin{array}{l}\text { Water } \\
\text { Used }\end{array}$ & $\begin{array}{l}\text { Time } \\
\text { min }\end{array}$ & Reading & $\begin{array}{c}\text { Flow } \\
\text { Reading, cm }\end{array}$ & $\begin{array}{l}\text { Flow, } \\
\mathrm{cm} / \mathrm{min}\end{array}$ & $\begin{array}{r}\begin{array}{l}V_{\mathrm{IR}}, \\
\mathrm{cm}^{3}\end{array}\end{array}$ & $\begin{array}{l}A_{\mathrm{IR}_{2}} \\
\mathrm{~cm}^{2}\end{array}$ & $t, \min$ & $V_{\mathrm{IR}}, \mathrm{cm} / \mathrm{h}$ & $V_{\mathrm{IR}}, \mathrm{cm} / \mathrm{s}$ \\
\hline $\begin{array}{c}\text { CR Field } 1 \\
\text { DR 6-2 }\end{array}$ & $\begin{array}{l}\text { CR- } \\
\text { CBM }\end{array}$ & 1 & 1 & 3.8 & 3.80 & 240.5 & 720 & 1 & 20.045 & $5.57 \mathrm{E}-03$ \\
\hline $\begin{array}{c}\text { CR Field } 1 \\
\text { DR 6-2 }\end{array}$ & $\begin{array}{l}\text { CR- } \\
\text { CBM }\end{array}$ & 2 & 2 & 4.20 & 4.20 & 265.9 & 720 & 1 & 22.155 & $6.15 \mathrm{E}-03$ \\
\hline $\begin{array}{c}\text { CR Field } 1 \\
\text { DR 6-2 }\end{array}$ & $\begin{array}{l}\text { CR- } \\
\text { CBM }\end{array}$ & 3 & 3 & 2.2 & 2.20 & 139.3 & 720 & 1 & 11.605 & $3.22 \mathrm{E}-03$ \\
\hline $\begin{array}{c}\text { CR Field } 1 \\
\text { DR 6-2 }\end{array}$ & $\begin{array}{l}\text { CR- } \\
\text { CBM }\end{array}$ & 5 & 4 & 2.9 & 1.45 & 183.6 & 720 & 2 & 7.649 & 2.12E-03 \\
\hline
\end{tabular}




\begin{tabular}{|c|c|c|c|c|c|c|c|c|c|c|}
\hline $\begin{array}{c}\text { CR Field } 1 \\
\text { DR 6-2 }\end{array}$ & $\begin{array}{l}\text { CR- } \\
\text { CBM }\end{array}$ & 9 & 5 & 8.4 & 2.10 & 531.7 & 720 & 4 & 11.078 & 3.08E-03 \\
\hline $\begin{array}{c}\text { CR Field } 1 \\
\text { DR 6-2 }\end{array}$ & $\begin{array}{c}\text { CR- } \\
\text { CBM }\end{array}$ & 19 & 6 & 24.5 & 2.45 & 1550.9 & 720 & 10 & 12.924 & $3.59 \mathrm{E}-03$ \\
\hline $\begin{array}{c}\text { CR Field } 1 \\
\text { DR 6-2 }\end{array}$ & $\begin{array}{l}\text { CR- } \\
\text { CBM }\end{array}$ & 20 & 7 & 2.5 & 2.50 & 158.3 & 720 & 1 & 13.188 & 3.66E-03 \\
\hline $\begin{array}{c}\text { CR Field } 1 \\
\text { DR 6-2 }\end{array}$ & $\begin{array}{l}\text { CR- } \\
\text { CBM }\end{array}$ & 21 & 8 & 1.6 & 1.60 & 101.3 & 720 & 1 & 8.440 & $2.34 \mathrm{E}-03$ \\
\hline $\begin{array}{c}\text { CR Field } 1 \\
\text { DR 6-2 }\end{array}$ & $\begin{array}{l}\text { CR- } \\
\text { CBM }\end{array}$ & 22 & 9 & 2.6 & 2.60 & 164.6 & 720 & 1 & 13.715 & 3.81E-03 \\
\hline $\begin{array}{c}\text { CR Field } 1 \\
\text { DR 6-2 }\end{array}$ & $\begin{array}{l}\text { CR- } \\
\text { CBM }\end{array}$ & 24 & 10 & 6 & 3.00 & 379.8 & 720 & 2 & 15.825 & $4.40 \mathrm{E}-03$ \\
\hline $\begin{array}{c}\text { CR Field } 1 \\
\text { DR 6-2 }\end{array}$ & $\begin{array}{l}\text { CR- } \\
\text { CBM }\end{array}$ & 29 & 11 & 14.1 & 2.82 & 892.5 & 720 & 5 & 14.876 & 4.13E-03 \\
\hline $\begin{array}{c}\text { CR Field } 1 \\
\text { DR 6-2 }\end{array}$ & $\begin{array}{l}\text { CR- } \\
\text { CBM }\end{array}$ & 39 & 12 & 16.1 & 1.61 & 1019.1 & 720 & 10 & 8.493 & $2.36 \mathrm{E}-03$ \\
\hline $\begin{array}{c}\text { CR Field } 1 \\
\text { DR 6-2 }\end{array}$ & $\begin{array}{l}\text { CR- } \\
\text { CBM }\end{array}$ & 43 & 13 & 8.3 & 2.08 & 525.4 & 720 & 4 & 10.946 & 3.04E-03 \\
\hline $\begin{array}{c}\text { CR Field } 1 \\
\text { DR 6-2 }\end{array}$ & $\begin{array}{l}\text { CR- } \\
\text { CBM }\end{array}$ & 55 & 15 & 16 & 1.33 & 1012.8 & 720 & 12 & 7.033 & $1.95 \mathrm{E}-03$ \\
\hline $\begin{array}{c}\text { CR Field } 1 \\
\text { DR 6-2 }\end{array}$ & $\begin{array}{l}\text { CR- } \\
\text { CBM }\end{array}$ & 59 & 16 & 25.7 & 6.43 & 1626.8 & 720 & 4 & 33.892 & $9.41 \mathrm{E}-03$ \\
\hline $\begin{array}{c}\text { CR Field } 1 \\
\text { DR 6-2 }\end{array}$ & $\begin{array}{l}\text { CR- } \\
\text { CBM }\end{array}$ & 62 & 17 & 6.7 & 2.23 & 424.1 & 720 & 3 & 11.781 & $3.27 \mathrm{E}-03$ \\
\hline $\begin{array}{c}\text { CR Field } 1 \\
\text { DR 6-2 }\end{array}$ & $\begin{array}{l}\text { CR- } \\
\text { CBM }\end{array}$ & 64 & 18 & 1.4 & 0.70 & 88.6 & 720 & 2 & 3.693 & $1.03 \mathrm{E}-03$ \\
\hline $\begin{array}{c}\text { CR Field } 1 \\
\text { DR 6-2 }\end{array}$ & $\begin{array}{l}\text { CR- } \\
\text { CBM }\end{array}$ & 65 & 19 & 2.6 & 2.60 & 164.6 & 720 & 1 & 13.715 & 3.81E-03 \\
\hline $\begin{array}{c}\text { CR Field } 1 \\
\text { DR 6-2 }\end{array}$ & $\begin{array}{c}\text { CR- } \\
\text { CBM }\end{array}$ & 70 & 20 & 11.4 & 2.28 & 721.6 & 720 & 5 & 12.027 & 3.34E-03 \\
\hline $\begin{array}{c}\text { CR Field } 1 \\
\text { DR 6-2 }\end{array}$ & $\begin{array}{l}\text { CR- } \\
\text { CBM }\end{array}$ & 74 & 21 & 7.6 & 1.90 & 481.1 & 720 & 4 & 10.023 & $2.78 \mathrm{E}-03$ \\
\hline $\begin{array}{c}\text { CR Field } 1 \\
\text { DR 6-2 }\end{array}$ & $\begin{array}{l}\text { CR- } \\
\text { CBM }\end{array}$ & 78 & 22 & 7.3 & 1.83 & 462.1 & 720 & 4 & 9.627 & 2.67E-03 \\
\hline $\begin{array}{c}\text { CR Field } 1 \\
\text { DR 6-2 }\end{array}$ & $\begin{array}{l}\text { CR- } \\
\text { CBM }\end{array}$ & 85 & 23 & 12.7 & 1.81 & 803.9 & 720 & 7 & 9.570 & $2.66 \mathrm{E}-03$ \\
\hline $\begin{array}{c}\text { CR Field } 1 \\
\text { DR 6-2 }\end{array}$ & $\begin{array}{l}\text { CR- } \\
\text { CBM }\end{array}$ & 92 & 26 & 2.5 & 0.36 & 158.3 & 720 & 7 & 1.884 & $5.23 \mathrm{E}-04$ \\
\hline $\begin{array}{c}\text { CR Field } 1 \\
\text { DR 6-2 }\end{array}$ & $\begin{array}{l}\text { CR- } \\
\text { CBM }\end{array}$ & 93 & 27 & 1.9 & 1.90 & 120.3 & 720 & 1 & 10.023 & $2.78 \mathrm{E}-03$ \\
\hline $\begin{array}{c}\text { CR Field } 1 \\
\text { DR 6-2 }\end{array}$ & $\begin{array}{l}\text { CR- } \\
\text { CBM }\end{array}$ & 97 & 28 & 10.3 & 2.58 & 652.0 & 720 & 4 & 13.583 & 3.77E-03 \\
\hline $\begin{array}{c}\text { CR Field } 1 \\
\text { DR 6-2 }\end{array}$ & $\begin{array}{l}\text { CR- } \\
\text { CBM }\end{array}$ & 104 & 29 & 16.5 & 2.36 & 1044.5 & 720 & 7 & 12.434 & 3.45E-03 \\
\hline $\begin{array}{c}\text { CR Field } 1 \\
\text { DR 6-2 }\end{array}$ & $\begin{array}{l}\text { CR- } \\
\text { CBM }\end{array}$ & 110 & 30 & 13.9 & 2.32 & 879.9 & 720 & 6 & 12.220 & 3.39E-03 \\
\hline $\begin{array}{c}\text { CR Field } 1 \\
\text { DR 6-2 }\end{array}$ & $\begin{array}{l}\text { CR- } \\
\text { CBM }\end{array}$ & 112 & 31 & 5.1 & 2.55 & 322.8 & 720 & 2 & 13.451 & 3.74E-03 \\
\hline & & & & & & & & $\begin{array}{r}\text { Final } \\
\text { Infiltration } \\
\text { Rate: }\end{array}$ & 12.92 & 3.59E-03 \\
\hline & & & & & & & & & & \\
\hline & & & & & & & & & & \\
\hline \multirow{2}{*}{\multicolumn{11}{|c|}{$\begin{array}{l}6 / 19 / 2003 \\
\text { First Test }\end{array}$}} \\
\hline & & & & & & & & & & \\
\hline Test ID & Water & Time & Reading & Flow & Flow, & $V_{\mathbb{I R}}$ & $A_{\mathrm{IR}}$ & $t, \min$ & $V_{\mathrm{IR}}, \mathrm{cm} / \mathrm{h}$ & $V_{\mathrm{IR}}, \mathrm{cm} / \mathrm{s}$ \\
\hline
\end{tabular}




\begin{tabular}{|c|c|c|c|c|c|c|c|c|c|c|}
\hline & Used & $\min$ & & Reading, cm & $\mathrm{cm} / \mathrm{min}$ & $\mathrm{cm}^{3}$ & $\mathrm{~cm}^{2}$ & & & \\
\hline $\begin{array}{c}\text { CR Field } 1 \\
\text { DR 7-1 }\end{array}$ & $\begin{array}{l}\text { Piney } \\
\text { Creek }\end{array}$ & 1 & 1 & 5.70 & 5.70 & 360.8 & 730 & 1 & 29.656 & 8.24E-03 \\
\hline $\begin{array}{c}\text { CR Field } 1 \\
\text { DR 7-1 }\end{array}$ & $\begin{array}{l}\text { Piney } \\
\text { Creek }\end{array}$ & 2 & 2 & 7.2 & 7.20 & 455.8 & 730 & 1 & 37.460 & $1.04 \mathrm{E}-02$ \\
\hline $\begin{array}{c}\text { CR Field } 1 \\
\text { DR 7-1 }\end{array}$ & $\begin{array}{l}\text { Piney } \\
\text { Creek }\end{array}$ & 3 & 3 & 2.5 & 2.50 & 158.3 & 730 & 1 & 13.007 & $3.61 \mathrm{E}-03$ \\
\hline $\begin{array}{c}\text { CR Field } 1 \\
\text { DR 7-1 }\end{array}$ & $\begin{array}{l}\text { Piney } \\
\text { Creek }\end{array}$ & 5 & 4 & 4.8 & 2.40 & 303.8 & 730 & 2 & 12.487 & $3.47 \mathrm{E}-03$ \\
\hline $\begin{array}{c}\text { CR Field } 1 \\
\text { DR 7-1 }\end{array}$ & $\begin{array}{l}\text { Piney } \\
\text { Creek }\end{array}$ & 7 & 5 & 6.7 & 3.35 & 424.1 & 730 & 2 & 17.429 & $4.84 \mathrm{E}-03$ \\
\hline $\begin{array}{c}\text { CR Field } 1 \\
\text { DR 7-1 }\end{array}$ & $\begin{array}{l}\text { Piney } \\
\text { Creek }\end{array}$ & 10 & 6 & 9.8 & 3.27 & 620.3 & 730 & 3 & 16.996 & $4.72 \mathrm{E}-03$ \\
\hline $\begin{array}{c}\text { CR Field } 1 \\
\text { DR 7-1 }\end{array}$ & $\begin{array}{l}\text { Piney } \\
\text { Creek }\end{array}$ & 13 & 7 & 6.5 & 2.17 & 411.5 & 730 & 3 & 11.273 & 3.13E-03 \\
\hline $\begin{array}{c}\text { CR Field } 1 \\
\text { DR 7-1 }\end{array}$ & $\begin{array}{l}\text { Piney } \\
\text { Creek }\end{array}$ & 19 & 8 & 5.3 & 0.88 & 335.5 & 730 & 6 & 4.596 & $1.28 \mathrm{E}-03$ \\
\hline $\begin{array}{c}\text { CR Field } 1 \\
\text { DR 7-1 }\end{array}$ & $\begin{array}{l}\text { Piney } \\
\text { Creek }\end{array}$ & 21 & 10 & 0.8 & 0.40 & 50.6 & 730 & 2 & 2.081 & $5.78 \mathrm{E}-04$ \\
\hline $\begin{array}{c}\text { CR Field } 1 \\
\text { DR 7-1 }\end{array}$ & $\begin{array}{l}\text { Piney } \\
\text { Creek }\end{array}$ & 23 & 11 & 1.5 & 0.75 & 95.0 & 730 & 2 & 3.902 & $1.08 \mathrm{E}-03$ \\
\hline $\begin{array}{c}\text { CR Field } 1 \\
\text { DR 7-1 }\end{array}$ & $\begin{array}{l}\text { Piney } \\
\text { Creek }\end{array}$ & 27 & 12 & 6.2 & 1.55 & 392.5 & 730 & 4 & 8.064 & $2.24 \mathrm{E}-03$ \\
\hline $\begin{array}{c}\text { CR Field } 1 \\
\text { DR 7-1 }\end{array}$ & $\begin{array}{l}\text { Piney } \\
\text { Creek }\end{array}$ & 33 & 13 & 9.9 & 1.65 & 626.7 & 730 & 6 & 8.585 & $2.38 \mathrm{E}-03$ \\
\hline $\begin{array}{c}\text { CR Field } 1 \\
\text { DR 7-1 }\end{array}$ & $\begin{array}{l}\text { Piney } \\
\text { Creek }\end{array}$ & 39 & 14 & 8.9 & 1.48 & 563.4 & 730 & 6 & 7.717 & $2.14 \mathrm{E}-03$ \\
\hline $\begin{array}{c}\text { CR Field } 1 \\
\text { DR 7-1 }\end{array}$ & $\begin{array}{l}\text { Piney } \\
\text { Creek }\end{array}$ & 45 & 15 & 8 & 1.33 & 506.4 & 730 & 6 & 6.937 & $1.93 \mathrm{E}-03$ \\
\hline $\begin{array}{c}\text { CR Field } 1 \\
\text { DR 7-1 }\end{array}$ & $\begin{array}{l}\text { Piney } \\
\text { Creek }\end{array}$ & 55 & 16 & 8.8 & 0.88 & 557.0 & 730 & 10 & 4.578 & $1.27 \mathrm{E}-03$ \\
\hline $\begin{array}{c}\text { CR Field } 1 \\
\text { DR 7-1 }\end{array}$ & $\begin{array}{l}\text { Piney } \\
\text { Creek }\end{array}$ & 60 & 17 & 3.2 & 0.64 & 202.6 & 730 & 5 & 3.330 & 9.25E-04 \\
\hline $\begin{array}{c}\text { CR Field } 1 \\
\text { DR 7-1 }\end{array}$ & $\begin{array}{l}\text { Piney } \\
\text { Creek }\end{array}$ & 63 & 18 & 5 & 1.67 & 316.5 & 730 & 3 & 8.671 & $2.41 \mathrm{E}-03$ \\
\hline $\begin{array}{c}\text { CR Field } 1 \\
\text { DR 7-1 }\end{array}$ & $\begin{array}{l}\text { Piney } \\
\text { Creek }\end{array}$ & 65 & 19 & 2.8 & 1.40 & 177.2 & 730 & 2 & 7.284 & $2.02 \mathrm{E}-03$ \\
\hline $\begin{array}{c}\text { CR Field } 1 \\
\text { DR 7-1 }\end{array}$ & $\begin{array}{l}\text { Piney } \\
\text { Creek }\end{array}$ & 74 & 20 & 8.7 & 0.97 & 550.7 & 730 & 9 & 5.029 & $1.40 \mathrm{E}-03$ \\
\hline $\begin{array}{c}\text { CR Field } 1 \\
\text { DR 7-1 }\end{array}$ & $\begin{array}{l}\text { Piney } \\
\text { Creek }\end{array}$ & 82 & 21 & 6.8 & 0.85 & 430.4 & 730 & 8 & 4.422 & $1.23 \mathrm{E}-03$ \\
\hline $\begin{array}{c}\text { CR Field } 1 \\
\text { DR 7-1 }\end{array}$ & $\begin{array}{l}\text { Piney } \\
\text { Creek }\end{array}$ & 88 & 22 & 4.7 & 0.78 & 297.5 & 730 & 6 & 4.075 & $1.13 \mathrm{E}-03$ \\
\hline $\begin{array}{c}\text { CR Field } 1 \\
\text { DR 7-1 }\end{array}$ & $\begin{array}{l}\text { Piney } \\
\text { Creek }\end{array}$ & 93 & 23 & 4.6 & 0.92 & 291.2 & 730 & 5 & 4.787 & $1.33 \mathrm{E}-03$ \\
\hline $\begin{array}{c}\text { CR Field } 1 \\
\text { DR 7-1 }\end{array}$ & $\begin{array}{l}\text { Piney } \\
\text { Creek }\end{array}$ & 97 & 24 & 3.9 & 0.98 & 246.9 & 730 & 4 & 5.073 & $1.41 \mathrm{E}-03$ \\
\hline $\begin{array}{c}\text { CR Field } 1 \\
\text { DR 7-1 }\end{array}$ & $\begin{array}{l}\text { Piney } \\
\text { Creek }\end{array}$ & 99 & 25 & 1.8 & 0.90 & 113.9 & 730 & 2 & 4.682 & $1.30 \mathrm{E}-03$ \\
\hline \multirow[t]{4}{*}{$\begin{array}{c}\text { CR Field } 1 \\
\text { DR 7-1 }\end{array}$} & $\begin{array}{l}\text { Piney } \\
\text { Creek }\end{array}$ & 101 & 26 & 1.8 & 0.90 & 113.9 & 730 & 2 & 4.682 & $1.30 \mathrm{E}-03$ \\
\hline & & & & & & & & $\begin{array}{r}\text { Final } \\
\text { Infiltration } \\
\text { Rate: }\end{array}$ & 4.81 & $1.34 \mathrm{E}-03$ \\
\hline & & & & & & & & & & \\
\hline & & & & & & & & & & \\
\hline
\end{tabular}




\begin{tabular}{|c|c|c|c|c|c|c|c|c|c|c|}
\hline \multirow{2}{*}{\multicolumn{11}{|c|}{\begin{tabular}{|l|l|} 
& \\
\end{tabular}}} \\
\hline & & & & & & & & & & \\
\hline \multicolumn{11}{|l|}{ 6/19/2003 } \\
\hline \multicolumn{11}{|l|}{ First Test } \\
\hline Test ID & $\begin{array}{l}\text { Water } \\
\text { Used }\end{array}$ & $\begin{array}{c}\text { Time } \\
\text { min }\end{array}$ & Reading & $\begin{array}{c}\text { Flow } \\
\text { Reading, cm }\end{array}$ & $\begin{array}{l}\text { Flow, } \\
\mathrm{cm} / \mathrm{min}\end{array}$ & $\begin{array}{l}\begin{array}{l}V_{\text {IR }}, \\
\mathrm{cm}^{3}\end{array}\end{array}$ & $\begin{array}{l}A_{\mathrm{IR}}, \\
\mathrm{cm}^{2}\end{array}$ & $t, \min$ & $V_{\mathrm{IR}}, \mathrm{cm} / \mathrm{h}$ & $V_{\mathrm{IR}}, \mathrm{cm} / \mathrm{s}$ \\
\hline $\begin{array}{c}\text { CR Field } 1 \\
\text { DR 8-1 }\end{array}$ & $\begin{array}{l}\text { Piney } \\
\text { Creek }\end{array}$ & 2 & 1 & 3.00 & 1.50 & 189.9 & 730 & 2 & 7.804 & $2.17 \mathrm{E}-03$ \\
\hline $\begin{array}{c}\text { CR Field } 1 \\
\text { DR 8-1 }\end{array}$ & $\begin{array}{l}\text { Piney } \\
\text { Creek }\end{array}$ & 4 & 2 & 1.6 & 0.80 & 101.3 & 730 & 2 & 4.162 & $1.16 \mathrm{E}-03$ \\
\hline $\begin{array}{c}\text { CR Field } 1 \\
\text { DR 8-1 }\end{array}$ & $\begin{array}{l}\text { Piney } \\
\text { Creek }\end{array}$ & 7 & 3 & 3.8 & 1.27 & 240.5 & 730 & 3 & 6.590 & $1.83 \mathrm{E}-03$ \\
\hline $\begin{array}{l}\text { CR Field } 1 \\
\text { DR 8-1 }\end{array}$ & $\begin{array}{l}\text { Piney } \\
\text { Creek }\end{array}$ & 10 & 4 & 0.7 & 0.23 & 44.3 & 730 & 3 & 1.214 & 3.37E-04 \\
\hline $\begin{array}{c}\text { CR Field } 1 \\
\text { DR 8-1 }\end{array}$ & $\begin{array}{l}\text { Piney } \\
\text { Creek }\end{array}$ & 15 & 5 & 3.5 & 0.70 & 221.6 & 730 & 5 & 3.642 & $1.01 \mathrm{E}-03$ \\
\hline $\begin{array}{c}\text { CR Field } 1 \\
\text { DR 8-1 }\end{array}$ & $\begin{array}{l}\text { Piney } \\
\text { Creek }\end{array}$ & 20 & 6 & 2 & 0.40 & 126.6 & 730 & 5 & 2.081 & $5.78 \mathrm{E}-04$ \\
\hline $\begin{array}{c}\text { CR Field } 1 \\
\text { DR 8-1 }\end{array}$ & $\begin{array}{l}\text { Piney } \\
\text { Creek }\end{array}$ & 25 & 7 & 1.9 & 0.38 & 120.3 & 730 & 5 & 1.977 & $5.49 \mathrm{E}-04$ \\
\hline $\begin{array}{c}\text { CR Field } 1 \\
\text { DR 8-1 }\end{array}$ & $\begin{array}{l}\text { Piney } \\
\text { Creek }\end{array}$ & 30 & 8 & 4 & 0.80 & 253.2 & 730 & 5 & 4.162 & $1.16 \mathrm{E}-03$ \\
\hline $\begin{array}{c}\text { CR Field } 1 \\
\text { DR 8-1 }\end{array}$ & $\begin{array}{l}\text { Piney } \\
\text { Creek }\end{array}$ & 40 & 9 & 3.5 & 0.35 & 221.6 & 730 & 10 & 1.821 & $5.06 \mathrm{E}-04$ \\
\hline $\begin{array}{c}\text { CR Field } 1 \\
\text { DR 8-1 }\end{array}$ & $\begin{array}{l}\text { Piney } \\
\text { Creek }\end{array}$ & 45 & 10 & 1.6 & 0.32 & 101.3 & 730 & 5 & 1.665 & $4.62 \mathrm{E}-04$ \\
\hline $\begin{array}{c}\text { CR Field } 1 \\
\text { DR 8-1 }\end{array}$ & $\begin{array}{l}\text { Piney } \\
\text { Creek }\end{array}$ & 50 & 11 & 1 & 0.20 & 63.3 & 730 & 5 & 1.041 & $2.89 \mathrm{E}-04$ \\
\hline $\begin{array}{c}\text { CR Field } 1 \\
\text { DR 8-1 }\end{array}$ & $\begin{array}{l}\text { Piney } \\
\text { Creek }\end{array}$ & 55 & 12 & 1 & 0.20 & 63.3 & 730 & 5 & 1.041 & 2.89E-04 \\
\hline $\begin{array}{c}\text { CR Field } 1 \\
\text { DR 8-1 }\end{array}$ & $\begin{array}{l}\text { Piney } \\
\text { Creek }\end{array}$ & 60 & 13 & 1.2 & 0.24 & 76.0 & 730 & 5 & 1.249 & $3.47 \mathrm{E}-04$ \\
\hline $\begin{array}{c}\text { CR Field } 1 \\
\text { DR 8-1 }\end{array}$ & $\begin{array}{l}\text { Piney } \\
\text { Creek }\end{array}$ & 65 & 14 & 1.4 & 0.28 & 88.6 & 730 & 5 & 1.457 & 4.05E-04 \\
\hline \multirow[t]{2}{*}{$\begin{array}{c}\text { CR Field } 1 \\
\text { DR 8-1 }\end{array}$} & $\begin{array}{l}\text { Piney } \\
\text { Creek }\end{array}$ & 70 & 15 & 1.1 & 0.22 & 69.6 & 730 & 5 & 1.145 & 3.18E-04 \\
\hline & & & & & & & & $\begin{array}{r}\text { Final } \\
\text { Infiltration } \\
\text { Rate: }\end{array}$ & 1.27 & $3.52 \mathrm{E}-04$ \\
\hline & & & & & & & & & & \\
\hline & & & & & & & & & & \\
\hline & & & & & & & & & & \\
\hline & & & & & & & & & & \\
\hline \multicolumn{11}{|l|}{ 6/19/2003 } \\
\hline \multicolumn{11}{|l|}{$\begin{array}{l}\text { 2nd Test- } \\
\text { Same } \\
\text { location }\end{array}$} \\
\hline Test ID & $\begin{array}{l}\text { Water } \\
\text { Used }\end{array}$ & $\begin{array}{c}\text { Time } \\
\text { min }\end{array}$ & Reading & $\begin{array}{c}\text { Flow } \\
\text { Reading, cm }\end{array}$ & $\begin{array}{l}\text { Flow, } \\
\mathrm{cm} / \mathrm{min}\end{array}$ & $\begin{array}{r}\begin{array}{l}V_{\text {IR }}, \\
\mathrm{cm}^{3}\end{array} \\
\mathrm{cos}^{2}\end{array}$ & $\begin{array}{l}A_{\mathrm{IR}}, \\
\mathrm{cm}^{2}\end{array}$ & $t, \min$ & $V_{\mathrm{IR}}, \mathrm{cm} / \mathrm{h}$ & $V_{\mathrm{IR}}, \mathrm{cm} / \mathrm{s}$ \\
\hline $\begin{array}{c}\text { CR Field } 1 \\
\text { DR 7-2 }\end{array}$ & $\begin{array}{l}\text { Piney } \\
\text { Creek }\end{array}$ & 1 & 1 & 1 & 1.00 & 63.3 & 725 & 1 & 5.239 & $1.46 \mathrm{E}-03$ \\
\hline $\begin{array}{l}\text { CR Field } 1 \\
\text { DR } 7-2\end{array}$ & $\begin{array}{l}\text { Piney } \\
\text { Creek }\end{array}$ & 2 & 2 & 0.80 & 0.80 & 50.6 & 725 & 1 & 4.191 & $1.16 \mathrm{E}-03$ \\
\hline $\begin{array}{c}\text { CR Field } 1 \\
\text { DR 7-2 }\end{array}$ & $\begin{array}{l}\text { Piney } \\
\text { Creek }\end{array}$ & 3 & 3 & 0.8 & 0.80 & 50.6 & 725 & 1 & 4.191 & $1.16 \mathrm{E}-03$ \\
\hline CR Field 1 & Piney & 5 & 4 & 2.9 & 1.45 & 183.6 & 725 & 2 & 7.596 & $2.11 \mathrm{E}-03$ \\
\hline
\end{tabular}




\begin{tabular}{|c|c|c|c|c|c|c|c|c|c|c|}
\hline DR 7-2 & Creek & & & & & & & & & \\
\hline $\begin{array}{c}\text { CR Field } 1 \\
\text { DR 7-2 }\end{array}$ & $\begin{array}{l}\text { Piney } \\
\text { Creek }\end{array}$ & 11 & 5 & 11.4 & 1.90 & 721.6 & 725 & 6 & 9.953 & $2.76 \mathrm{E}-03$ \\
\hline $\begin{array}{c}\text { CR Field } 1 \\
\text { DR 7-2 }\end{array}$ & $\begin{array}{l}\text { Piney } \\
\text { Creek }\end{array}$ & 17 & 6 & 7.4 & 1.23 & 468.4 & 725 & 6 & 6.461 & $1.79 \mathrm{E}-03$ \\
\hline $\begin{array}{c}\text { CR Field } 1 \\
\text { DR 7-2 }\end{array}$ & $\begin{array}{l}\text { Piney } \\
\text { Creek }\end{array}$ & 23 & 7 & 9.5 & 1.58 & 601.4 & 725 & 6 & 8.294 & $2.30 \mathrm{E}-03$ \\
\hline $\begin{array}{c}\text { CR Field } 1 \\
\text { DR 7-2 }\end{array}$ & $\begin{array}{l}\text { Piney } \\
\text { Creek }\end{array}$ & 29 & 8 & 11.2 & 1.87 & 709.0 & 725 & 6 & 9.779 & $2.72 \mathrm{E}-03$ \\
\hline $\begin{array}{c}\text { CR Field } 1 \\
\text { DR 7-2 }\end{array}$ & $\begin{array}{l}\text { Piney } \\
\text { Creek }\end{array}$ & 30 & 9 & 2.3 & 2.30 & 145.6 & 725 & 1 & 12.049 & $3.35 \mathrm{E}-03$ \\
\hline $\begin{array}{c}\text { CR Field } 1 \\
\text { DR 7-2 }\end{array}$ & $\begin{array}{l}\text { Piney } \\
\text { Creek }\end{array}$ & 32 & 11 & 0.4 & 0.20 & 25.3 & 725 & 2 & 1.048 & $2.91 \mathrm{E}-04$ \\
\hline $\begin{array}{c}\text { CR Field } 1 \\
\text { DR 7-2 }\end{array}$ & $\begin{array}{l}\text { Piney } \\
\text { Creek }\end{array}$ & 34 & 12 & 0.9 & 0.45 & 57.0 & 725 & 2 & 2.357 & $6.55 \mathrm{E}-04$ \\
\hline $\begin{array}{c}\text { CR Field } 1 \\
\text { DR 7-2 }\end{array}$ & $\begin{array}{l}\text { Piney } \\
\text { Creek }\end{array}$ & 38 & 13 & 3.4 & 0.85 & 215.2 & 725 & 4 & 4.453 & $1.24 \mathrm{E}-03$ \\
\hline $\begin{array}{c}\text { CR Field } 1 \\
\text { DR 7-2 }\end{array}$ & $\begin{array}{l}\text { Piney } \\
\text { Creek }\end{array}$ & 46 & 14 & 23.7 & 2.96 & 1500.2 & 725 & 8 & 15.519 & $4.31 \mathrm{E}-03$ \\
\hline $\begin{array}{c}\text { CR Field } 1 \\
\text { DR 7-2 }\end{array}$ & $\begin{array}{l}\text { Piney } \\
\text { Creek }\end{array}$ & 50 & 15 & 6.3 & 1.58 & 398.8 & 725 & 4 & 8.251 & $2.29 \mathrm{E}-03$ \\
\hline $\begin{array}{c}\text { CR Field } 1 \\
\text { DR 7-2 }\end{array}$ & $\begin{array}{l}\text { Piney } \\
\text { Creek }\end{array}$ & 54 & 16 & 6.7 & 1.68 & 424.1 & 725 & 4 & 8.775 & $2.44 \mathrm{E}-03$ \\
\hline $\begin{array}{c}\text { CR Field } 1 \\
\text { DR 7-2 }\end{array}$ & $\begin{array}{l}\text { Piney } \\
\text { Creek }\end{array}$ & 58 & 17 & 7.4 & 1.85 & 468.4 & 725 & 4 & 9.691 & $2.69 \mathrm{E}-03$ \\
\hline $\begin{array}{c}\text { CR Field } 1 \\
\text { DR 7-2 }\end{array}$ & $\begin{array}{l}\text { Piney } \\
\text { Creek }\end{array}$ & 60 & 19 & 1.4 & 0.70 & 88.6 & 725 & 2 & 3.667 & $1.02 \mathrm{E}-03$ \\
\hline $\begin{array}{c}\text { CR Field } 1 \\
\text { DR 7-2 }\end{array}$ & $\begin{array}{l}\text { Piney } \\
\text { Creek }\end{array}$ & 64 & 20 & 7 & 1.75 & 443.1 & 725 & 4 & 9.168 & $2.55 \mathrm{E}-03$ \\
\hline $\begin{array}{c}\text { CR Field } 1 \\
\text { DR 7-2 }\end{array}$ & $\begin{array}{l}\text { Piney } \\
\text { Creek }\end{array}$ & 68 & 21 & 7 & 1.75 & 443.1 & 725 & 4 & 9.168 & $2.55 \mathrm{E}-03$ \\
\hline $\begin{array}{c}\text { CR Field } 1 \\
\text { DR 7-2 }\end{array}$ & $\begin{array}{l}\text { Piney } \\
\text { Creek }\end{array}$ & 72 & 22 & 6.6 & 1.65 & 417.8 & 725 & 4 & 8.644 & $2.40 \mathrm{E}-03$ \\
\hline $\begin{array}{c}\text { CR Field } 1 \\
\text { DR 7-2 }\end{array}$ & $\begin{array}{l}\text { Piney } \\
\text { Creek }\end{array}$ & 76 & 23 & 7.5 & 1.88 & 474.8 & 725 & 4 & 9.822 & $2.73 \mathrm{E}-03$ \\
\hline & & & & & & & & $\begin{array}{r}\text { Final } \\
\text { Infiltration } \\
\text { Rate: }\end{array}$ & 9.20 & $2.56 \mathrm{E}-03$ \\
\hline & & & & & & & & & & \\
\hline & & & & & & & & & & \\
\hline & & & & & & & & & & \\
\hline \multicolumn{11}{|l|}{$6 / 19 / 2003$} \\
\hline \multicolumn{11}{|l|}{\begin{tabular}{|l|} 
2nd Test- \\
Same \\
location \\
\end{tabular}} \\
\hline Test ID & $\begin{array}{l}\text { Water } \\
\text { Used }\end{array}$ & $\begin{array}{c}\text { Time } \\
\text { min }\end{array}$ & Reading & $\begin{array}{c}\text { Flow } \\
\text { Reading, cm }\end{array}$ & $\begin{array}{l}\text { Flow, } \\
\mathrm{cm} / \mathrm{min}\end{array}$ & $\begin{array}{r}\begin{array}{r}V_{\text {IR }}, \\
\mathrm{cm}^{3}\end{array} \\
\end{array}$ & $\begin{array}{l}A_{\mathrm{IR}}, \\
\mathrm{cm}^{2}\end{array}$ & $t, \min$ & $V_{\mathrm{IR}}, \mathrm{cm} / \mathrm{h}$ & $V_{\mathrm{IR}}, \mathrm{cm} / \mathrm{s}$ \\
\hline $\begin{array}{c}\text { CR Field } 1 \\
\text { DR 8-2 }\end{array}$ & $\begin{array}{c}\text { CR- } \\
\text { CBM }\end{array}$ & 1 & 1 & 3.8 & 3.80 & 240.5 & 725 & 1 & 19.907 & 5.53E-03 \\
\hline $\begin{array}{c}\text { CR Field } 1 \\
\text { DR 8-2 }\end{array}$ & $\begin{array}{l}\text { CR- } \\
\text { CBM }\end{array}$ & 2 & 2 & 7.90 & 7.90 & 500.1 & 725 & 1 & 41.385 & $1.15 \mathrm{E}-02$ \\
\hline $\begin{array}{c}\text { CR Field } 1 \\
\text { DR 8-2 }\end{array}$ & $\begin{array}{l}\text { CR- } \\
\text { CBM }\end{array}$ & 4 & 3 & 9 & 4.50 & 569.7 & 725 & 2 & 23.574 & $6.55 \mathrm{E}-03$ \\
\hline $\begin{array}{c}\text { CR Field } 1 \\
\text { DR 8-2 }\end{array}$ & $\begin{array}{l}\text { CR- } \\
\text { CBM }\end{array}$ & 9 & 4 & 22.5 & 4.50 & 1424.3 & 725 & 5 & 23.574 & $6.55 \mathrm{E}-03$ \\
\hline CR Field 1 & CR- & 12 & 5 & 4 & 1.33 & 253.2 & 725 & 3 & 6.985 & $1.94 \mathrm{E}-03$ \\
\hline
\end{tabular}




\begin{tabular}{|c|c|c|c|c|c|c|c|c|c|c|}
\hline DR 8-2 & CBM & & & & & & & & & \\
\hline $\begin{array}{c}\text { CR Field } 1 \\
\text { DR 8-2 }\end{array}$ & $\begin{array}{l}\text { CR- } \\
\text { CBM }\end{array}$ & 17 & 6 & 4.4 & 0.88 & 278.5 & 725 & 5 & 4.610 & $1.28 \mathrm{E}-03$ \\
\hline $\begin{array}{c}\text { CR Field } 1 \\
\text { DR 8-2 }\end{array}$ & $\begin{array}{l}\text { CR- } \\
\text { CBM }\end{array}$ & 22 & 7 & 3.3 & 0.66 & 208.9 & 725 & 5 & 3.457 & $9.60 \mathrm{E}-04$ \\
\hline $\begin{array}{c}\text { CR Field } 1 \\
\text { DR 8-2 }\end{array}$ & $\begin{array}{l}\text { CR- } \\
\text { CBM }\end{array}$ & 27 & 8 & 2.2 & 0.44 & 139.3 & 725 & 5 & 2.305 & $6.40 \mathrm{E}-04$ \\
\hline $\begin{array}{c}\text { CR Field } 1 \\
\text { DR 8-2 }\end{array}$ & $\begin{array}{l}\text { CR- } \\
\text { CBM }\end{array}$ & 32 & 9 & 3.3 & 0.66 & 208.9 & 725 & 5 & 3.457 & $9.60 \mathrm{E}-04$ \\
\hline $\begin{array}{c}\text { CR Field } 1 \\
\text { DR 8-2 }\end{array}$ & $\begin{array}{l}\text { CR- } \\
\text { CBM }\end{array}$ & 37 & 10 & 3.1 & 0.62 & 196.2 & 725 & 5 & 3.248 & $9.02 \mathrm{E}-04$ \\
\hline $\begin{array}{c}\text { CR Field } 1 \\
\text { DR 8-2 }\end{array}$ & $\begin{array}{l}\text { CR- } \\
\text { CBM }\end{array}$ & 42 & 11 & 2.9 & 0.58 & 183.6 & 725 & 5 & 3.038 & $8.44 \mathrm{E}-04$ \\
\hline $\begin{array}{c}\text { CR Field } 1 \\
\text { DR 8-2 }\end{array}$ & $\begin{array}{l}\text { CR- } \\
\text { CBM }\end{array}$ & 47 & 12 & 3 & 0.60 & 189.9 & 725 & 5 & 3.143 & 8.73E-04 \\
\hline \multirow[t]{2}{*}{$\begin{array}{c}\text { CR Field } 1 \\
\text { DR 8-2 }\end{array}$} & $\begin{array}{l}\text { CR- } \\
\text { CBM }\end{array}$ & 52 & 13 & 3 & 0.60 & 189.9 & 725 & $\Gamma^{\circ}$ & 3.143 & 8.73E-04 \\
\hline & & & & & & & & $\begin{array}{r}\text { Final } \\
\text { Infiltration } \\
\text { Rate: }\end{array}$ & 3.14 & 8.73E-04 \\
\hline & & & & & & & & & & \\
\hline \multirow{2}{*}{\multicolumn{11}{|c|}{$6 / 28 / 2003$}} \\
\hline & & & & & & & & & & \\
\hline \multicolumn{11}{|l|}{ First Test } \\
\hline Test ID & $\begin{array}{l}\text { Water } \\
\text { Used }\end{array}$ & $\begin{array}{l}\text { Time } \\
\text { min }\end{array}$ & Reading & $\begin{array}{c}\text { Flow } \\
\text { Reading, cm } \\
\end{array}$ & $\begin{array}{c}\text { Flow, } \\
\mathrm{cm} / \mathrm{min}\end{array}$ & $\begin{array}{l}\begin{array}{l}V_{\mathrm{IR}}, \\
\mathrm{cm}^{3}\end{array} \\
\end{array}$ & $\begin{array}{l}A_{\mathrm{IR}}, \\
\mathrm{cm}^{2}\end{array}$ & $t, \min$ & $V_{\mathrm{IR}}, \mathrm{cm} / \mathrm{h}$ & $V_{\mathrm{IR}}, \mathrm{cm} / \mathrm{s}$ \\
\hline $\begin{array}{c}\text { CR Field } 1 \\
\text { DR 9-1 }\end{array}$ & $\begin{array}{l}\text { Piney } \\
\text { Creek }\end{array}$ & 1 & 1 & 13.20 & 13.20 & 835.6 & 730 & 1 & 68.676 & $1.91 \mathrm{E}-02$ \\
\hline $\begin{array}{c}\text { CR Field } 1 \\
\text { DR 9-1 }\end{array}$ & $\begin{array}{l}\text { Piney } \\
\text { Creek }\end{array}$ & 2 & 2 & 7.9 & 7.90 & 500.1 & 730 & 1 & 41.102 & $1.14 \mathrm{E}-02$ \\
\hline $\begin{array}{c}\text { CR Field } 1 \\
\text { DR 9-1 }\end{array}$ & $\begin{array}{l}\text { Piney } \\
\text { Creek }\end{array}$ & 4 & 3 & 9.9 & 4.95 & 626.7 & 730 & 2 & 25.754 & 7.15E-03 \\
\hline $\begin{array}{c}\text { CR Field } 1 \\
\text { DR 9-1 }\end{array}$ & $\begin{array}{l}\text { Piney } \\
\text { Creek }\end{array}$ & 7 & 4 & 16.3 & 5.43 & 1031.8 & 730 & 3 & 28.268 & 7.85E-03 \\
\hline $\begin{array}{c}\text { CR Field } 1 \\
\text { DR 9-1 }\end{array}$ & $\begin{array}{l}\text { Piney } \\
\text { Creek }\end{array}$ & 16 & 5 & 37.4 & 4.16 & 2367.4 & 730 & 9 & 21.620 & $6.01 \mathrm{E}-03$ \\
\hline $\begin{array}{c}\text { CR Field } 1 \\
\text { DR 9-1 }\end{array}$ & $\begin{array}{l}\text { Piney } \\
\text { Creek }\end{array}$ & 20 & 6 & 10.9 & 2.73 & 690.0 & 730 & 4 & 14.177 & $3.94 \mathrm{E}-03$ \\
\hline $\begin{array}{c}\text { CR Field } 1 \\
\text { DR 9-1 }\end{array}$ & $\begin{array}{l}\text { Piney } \\
\text { Creek }\end{array}$ & 23 & 7 & 7.1 & 2.37 & 449.4 & 730 & 3 & 12.313 & $3.42 \mathrm{E}-03$ \\
\hline $\begin{array}{c}\text { CR Field } 1 \\
\text { DR 9-1 }\end{array}$ & $\begin{array}{l}\text { Piney } \\
\text { Creek }\end{array}$ & 28 & 8 & 17.7 & 3.54 & 1120.4 & 730 & 5 & 18.418 & $5.12 \mathrm{E}-03$ \\
\hline $\begin{array}{c}\text { CR Field } 1 \\
\text { DR 9-1 }\end{array}$ & $\begin{array}{l}\text { Piney } \\
\text { Creek }\end{array}$ & 33 & 9 & 12.5 & 2.50 & 791.3 & 730 & 5 & 13.007 & $3.61 \mathrm{E}-03$ \\
\hline $\begin{array}{c}\text { CR Field } 1 \\
\text { DR 9-1 }\end{array}$ & $\begin{array}{l}\text { Piney } \\
\text { Creek }\end{array}$ & 38 & 10 & 13.6 & 2.72 & 860.9 & 730 & 5 & 14.151 & 3.93E-03 \\
\hline $\begin{array}{c}\text { CR Field } 1 \\
\text { DR 9-1 }\end{array}$ & $\begin{array}{l}\text { Piney } \\
\text { Creek }\end{array}$ & 40 & 11 & 3.8 & 1.90 & 240.5 & 730 & 2 & 9.885 & $2.75 \mathrm{E}-03$ \\
\hline $\begin{array}{c}\text { CR Field } 1 \\
\text { DR 9-1 }\end{array}$ & $\begin{array}{l}\text { Piney } \\
\text { Creek }\end{array}$ & 45 & 12 & 7.4 & 1.48 & 468.4 & 730 & 5 & 7.700 & $2.14 \mathrm{E}-03$ \\
\hline $\begin{array}{c}\text { CR Field } 1 \\
\text { DR 9-1 }\end{array}$ & $\begin{array}{l}\text { Piney } \\
\text { Creek }\end{array}$ & 50 & 13 & 10.9 & 2.18 & 690.0 & 730 & 5 & 11.342 & $3.15 \mathrm{E}-03$ \\
\hline $\begin{array}{c}\text { CR Field } 1 \\
\text { DR 9-1 }\end{array}$ & $\begin{array}{l}\text { Piney } \\
\text { Creek }\end{array}$ & 55 & 14 & 10 & 2.00 & 633.0 & 730 & 5 & 10.405 & $2.89 \mathrm{E}-03$ \\
\hline $\begin{array}{c}\text { CR Field } 1 \\
\text { DR 9-1 }\end{array}$ & $\begin{array}{l}\text { Piney } \\
\text { Creek }\end{array}$ & 60 & 15 & 10.5 & 2.10 & 664.7 & 730 & 5 & 10.926 & 3.03E-03 \\
\hline
\end{tabular}




\begin{tabular}{|c|c|c|c|c|c|c|c|c|c|c|}
\hline & & & & & & & & $\begin{array}{r}\text { Final } \\
\text { Infiltration } \\
\text { Rate: } \\
\end{array}$ & 10.89 & 3.03E-03 \\
\hline \multirow{2}{*}{\multicolumn{11}{|c|}{ 6/29/2003 }} \\
\hline & & & & & & & & & & \\
\hline \multicolumn{11}{|l|}{$\begin{array}{l}\text { 2nd Test- } \\
\text { same } \\
\text { location }\end{array}$} \\
\hline Test ID & $\begin{array}{l}\text { Water } \\
\text { Used }\end{array}$ & $\begin{array}{c}\text { Time } \\
\text { min }\end{array}$ & Reading & $\begin{array}{c}\text { Flow } \\
\text { Reading, cm } \\
\end{array}$ & $\begin{array}{l}\text { Flow, } \\
\mathrm{cm} / \mathrm{min}\end{array}$ & $\begin{array}{l}\begin{array}{l}V_{\text {IR }}, \\
\mathrm{cm}^{3}\end{array} \\
\end{array}$ & $\begin{array}{l}A_{\mathrm{IR}}, \\
\mathrm{cm}^{2}\end{array}$ & $t, \min$ & $V_{\mathrm{IR}}, \mathrm{cm} / \mathrm{h}$ & $V_{\mathrm{IR}}, \mathrm{cm} / \mathrm{s}$ \\
\hline $\begin{array}{c}\text { CR Field } 1 \\
\text { DR 9-2 }\end{array}$ & $\begin{array}{l}\text { Piney } \\
\text { Creek }\end{array}$ & 1 & 1 & 0.50 & 0.50 & 31.7 & 725 & 1 & 2.619 & $7.28 \mathrm{E}-04$ \\
\hline $\begin{array}{c}\text { CR Field } 1 \\
\text { DR 9-2 } \\
\end{array}$ & $\begin{array}{l}\text { Piney } \\
\text { Creek }\end{array}$ & 2 & 2 & 0.6 & 0.60 & 38.0 & 725 & 1 & 3.143 & 8.73E-04 \\
\hline $\begin{array}{c}\text { CR Field } 1 \\
\text { DR 9-2 } \\
\end{array}$ & $\begin{array}{l}\text { Piney } \\
\text { Creek }\end{array}$ & 3 & 3 & 0.8 & 0.80 & 50.6 & 725 & 1 & 4.191 & $1.16 \mathrm{E}-03$ \\
\hline $\begin{array}{c}\text { CR Field } 1 \\
\text { DR 9-2 }\end{array}$ & $\begin{array}{l}\text { Piney } \\
\text { Creek }\end{array}$ & 4 & 4 & 1.5 & 1.50 & 95.0 & 725 & 1 & 7.858 & $2.18 \mathrm{E}-03$ \\
\hline $\begin{array}{c}\text { CR Field } 1 \\
\text { DR 9-2 }\end{array}$ & $\begin{array}{l}\text { Piney } \\
\text { Creek }\end{array}$ & 5 & 5 & 1.7 & 1.70 & 107.6 & 725 & 1 & 8.906 & $2.47 \mathrm{E}-03$ \\
\hline $\begin{array}{c}\text { CR Field } 1 \\
\text { DR 9-2 }\end{array}$ & $\begin{array}{l}\text { Piney } \\
\text { Creek }\end{array}$ & 10 & 6 & 10.5 & 2.10 & 664.7 & 725 & 5 & 11.001 & 3.06E-03 \\
\hline $\begin{array}{c}\text { CR Field } 1 \\
\text { DR 9-2 }\end{array}$ & $\begin{array}{l}\text { Piney } \\
\text { Creek }\end{array}$ & 12 & 7 & 3.7 & 1.85 & 234.2 & 725 & 2 & 9.691 & 2.69E-03 \\
\hline $\begin{array}{c}\text { CR Field } 1 \\
\text { DR 9-2 }\end{array}$ & $\begin{array}{l}\text { Piney } \\
\text { Creek }\end{array}$ & 15 & 8 & 4.8 & 1.60 & 303.8 & 725 & 3 & 8.382 & $2.33 \mathrm{E}-03$ \\
\hline $\begin{array}{c}\text { CR Field } 1 \\
\text { DR 9-2 }\end{array}$ & $\begin{array}{l}\text { Piney } \\
\text { Creek }\end{array}$ & 20 & 9 & 6.2 & 1.24 & 392.5 & 725 & 5 & 6.496 & $1.80 \mathrm{E}-03$ \\
\hline $\begin{array}{c}\text { CR Field } 1 \\
\text { DR 9-2 }\end{array}$ & $\begin{array}{l}\text { Piney } \\
\text { Creek }\end{array}$ & 25 & 10 & 5.9 & 1.18 & 373.5 & 725 & 5 & 6.182 & $1.72 \mathrm{E}-03$ \\
\hline $\begin{array}{c}\text { CR Field } 1 \\
\text { DR 9-2 }\end{array}$ & $\begin{array}{l}\text { Piney } \\
\text { Creek }\end{array}$ & 30 & 11 & 5.6 & 1.12 & 354.5 & 725 & 5 & 5.867 & $1.63 \mathrm{E}-03$ \\
\hline $\begin{array}{c}\text { CR Field } 1 \\
\text { DR 9-2 }\end{array}$ & $\begin{array}{l}\text { Piney } \\
\text { Creek }\end{array}$ & 35 & 12 & 5.8 & 1.16 & 367.1 & 725 & 5 & 6.077 & $1.69 \mathrm{E}-03$ \\
\hline $\begin{array}{c}\text { CR Field } 1 \\
\text { DR 9-2 }\end{array}$ & $\begin{array}{l}\text { Piney } \\
\text { Creek }\end{array}$ & 38 & 14 & 3.7 & 1.23 & 234.2 & 725 & 3 & 6.461 & $1.79 \mathrm{E}-03$ \\
\hline $\begin{array}{c}\text { CR Field } 1 \\
\text { DR 9-2 }\end{array}$ & $\begin{array}{l}\text { Piney } \\
\text { Creek }\end{array}$ & 40 & 15 & 3.5 & 1.75 & 221.6 & 725 & 2 & 9.168 & $2.55 \mathrm{E}-03$ \\
\hline $\begin{array}{l}\text { CR Field } 1 \\
\text { DR 9-2 }\end{array}$ & $\begin{array}{l}\text { Piney } \\
\text { Creek }\end{array}$ & 42 & 16 & 6.5 & 3.25 & 411.5 & 725 & 2 & 17.026 & 4.73E-03 \\
\hline $\begin{array}{c}\text { CR Field } 1 \\
\text { DR 9-2 }\end{array}$ & $\begin{array}{l}\text { Piney } \\
\text { Creek }\end{array}$ & 44 & 17 & 3 & 1.50 & 189.9 & 725 & 2 & 7.858 & $2.18 \mathrm{E}-03$ \\
\hline $\begin{array}{c}\text { CR Field } 1 \\
\text { DR 9-2 }\end{array}$ & $\begin{array}{l}\text { Piney } \\
\text { Creek }\end{array}$ & 46 & 18 & 2.9 & 1.45 & 183.6 & 725 & 2 & 7.596 & $2.11 \mathrm{E}-03$ \\
\hline $\begin{array}{c}\text { CR Field } 1 \\
\text { DR 9-2 }\end{array}$ & $\begin{array}{l}\text { Piney } \\
\text { Creek }\end{array}$ & 48 & 19 & 2.6 & 1.30 & 164.6 & 725 & 2 & 6.810 & $1.89 \mathrm{E}-03$ \\
\hline $\begin{array}{c}\text { CR Field } 1 \\
\text { DR 9-2 }\end{array}$ & $\begin{array}{l}\text { Piney } \\
\text { Creek }\end{array}$ & 50 & 20 & 2.9 & 1.45 & 183.6 & 725 & 2 & 7.596 & $2.11 \mathrm{E}-03$ \\
\hline $\begin{array}{c}\text { CR Field } 1 \\
\text { DR 9-2 }\end{array}$ & $\begin{array}{l}\text { Piney } \\
\text { Creek }\end{array}$ & 52 & 21 & 2.6 & 1.30 & 164.6 & 725 & 2 & 6.810 & $1.89 \mathrm{E}-03$ \\
\hline $\begin{array}{c}\text { CR Field } 1 \\
\text { DR 9-2 }\end{array}$ & $\begin{array}{l}\text { Piney } \\
\text { Creek }\end{array}$ & 54 & 22 & 2.7 & 1.35 & 170.9 & 725 & 2 & 7.072 & $1.96 \mathrm{E}-03$ \\
\hline \multirow[t]{2}{*}{$\begin{array}{c}\text { CR Field } 1 \\
\text { DR 9-2 }\end{array}$} & $\begin{array}{l}\text { Piney } \\
\text { Creek }\end{array}$ & 56 & 23 & 2.7 & 1.35 & 170.9 & 725 & 2 & 7.072 & $1.96 \mathrm{E}-03$ \\
\hline & & & & & & & & $\begin{array}{r}\text { Final } \\
\text { Infiltration } \\
\text { Rate: } \\
\end{array}$ & 7.16 & $1.99 \mathrm{E}-03$ \\
\hline
\end{tabular}




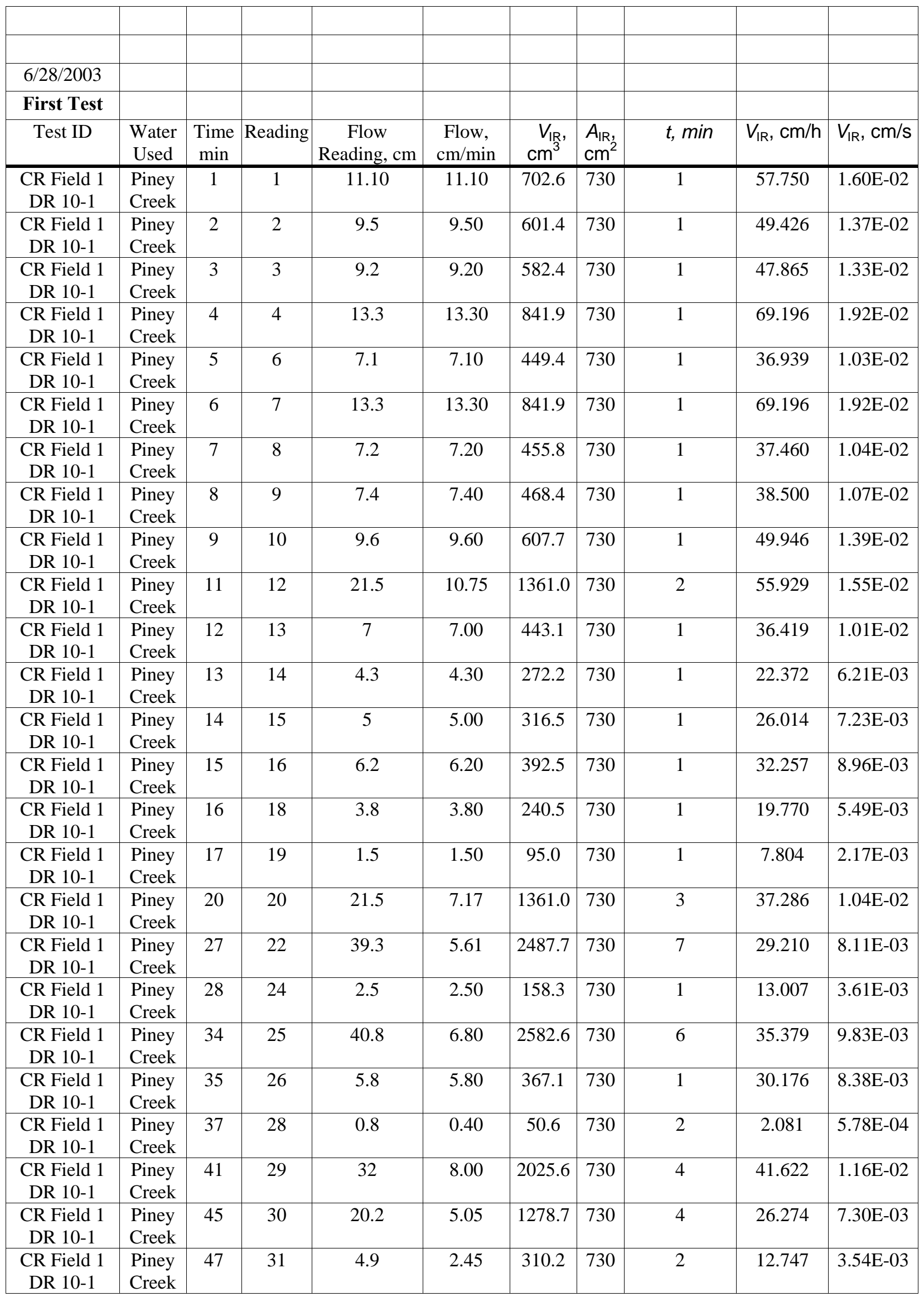




\begin{tabular}{|c|c|c|c|c|c|c|c|c|c|c|}
\hline $\begin{array}{c}\text { CR Field } 1 \\
\text { DR 10-1 }\end{array}$ & $\begin{array}{l}\text { Piney } \\
\text { Creek }\end{array}$ & 49 & 32 & 27.5 & 13.75 & 1740.8 & 730 & 2 & 71.538 & 1.99E-02 \\
\hline $\begin{array}{c}\text { CR Field } 1 \\
\text { DR 10-1 }\end{array}$ & $\begin{array}{l}\text { Piney } \\
\text { Creek }\end{array}$ & 51 & 33 & 8.5 & 4.25 & 538.1 & 730 & 2 & 22.112 & $6.14 \mathrm{E}-03$ \\
\hline $\begin{array}{c}\text { CR Field } 1 \\
\text { DR 10-1 }\end{array}$ & $\begin{array}{l}\text { Piney } \\
\text { Creek }\end{array}$ & 53 & 34 & 9.6 & 4.80 & 607.7 & 730 & 2 & 24.973 & $6.94 \mathrm{E}-03$ \\
\hline $\begin{array}{c}\text { CR Field } 1 \\
\text { DR 10-1 }\end{array}$ & $\begin{array}{l}\text { Piney } \\
\text { Creek }\end{array}$ & 55 & 36 & 5 & 2.50 & 316.5 & 730 & 2 & 13.007 & $3.61 \mathrm{E}-03$ \\
\hline $\begin{array}{c}\text { CR Field } 1 \\
\text { DR 10-1 }\end{array}$ & $\begin{array}{l}\text { Piney } \\
\text { Creek }\end{array}$ & 61 & 37 & 29.5 & 4.92 & 1867.4 & 730 & 6 & 25.580 & 7.11E-03 \\
\hline $\begin{array}{c}\text { CR Field } 1 \\
\text { DR 10-1 }\end{array}$ & $\begin{array}{l}\text { Piney } \\
\text { Creek }\end{array}$ & 63 & 38 & 9.6 & 4.80 & 607.7 & 730 & 2 & 24.973 & $6.94 \mathrm{E}-03$ \\
\hline $\begin{array}{c}\text { CR Field } 1 \\
\text { DR 10-1 }\end{array}$ & $\begin{array}{l}\text { Piney } \\
\text { Creek }\end{array}$ & 65 & 39 & 9.2 & 4.60 & 582.4 & 730 & 2 & 23.933 & 6.65E-03 \\
\hline & & & & & & & & $\begin{array}{r}\text { Final } \\
\text { Infiltration } \\
\text { Rate: }\end{array}$ & 24.83 & $6.90 \mathrm{E}-03$ \\
\hline & & & & & & & & & & \\
\hline & & & & & & & & & & \\
\hline $6 / 29 / 2003$ & & & & & & & & & & \\
\hline $\begin{array}{l}\text { 2nd Test- } \\
\text { same } \\
\text { location }\end{array}$ & & & & & & & & & & \\
\hline Test ID & $\begin{array}{l}\text { Water } \\
\text { Used }\end{array}$ & $\begin{array}{l}\text { Time } \\
\text { min }\end{array}$ & Reading & $\begin{array}{c}\text { Flow } \\
\text { Reading, } \mathrm{cm}\end{array}$ & $\begin{array}{c}\text { Flow, } \\
\mathrm{cm} / \mathrm{min}\end{array}$ & $\begin{array}{r}V_{\mathrm{IR}}, \\
\mathrm{cm}^{3}\end{array}$ & $\begin{array}{l}A_{\mathrm{IR}} \\
\mathrm{cm}^{2}\end{array}$ & $t, \min$ & $V_{\mathrm{IR}}, \mathrm{cm} / \mathrm{h}$ & $V_{\mathrm{IR}}, \mathrm{cm} / \mathrm{s}$ \\
\hline $\begin{array}{c}\text { CR Field } 1 \\
\text { DR 10-2 }\end{array}$ & $\begin{array}{l}\text { CR- } \\
\text { CBM }\end{array}$ & 1 & 1 & 0.80 & 0.80 & 50.6 & 725 & 1 & 4.191 & $1.16 \mathrm{E}-03$ \\
\hline $\begin{array}{c}\text { CR Field } 1 \\
\text { DR 10-2 }\end{array}$ & $\begin{array}{l}\text { CR- } \\
\text { CBM }\end{array}$ & 3 & 2 & 10.1 & 5.05 & 639.3 & 725 & 2 & 26.455 & 7.35E-03 \\
\hline $\begin{array}{c}\text { CR Field } 1 \\
\text { DR 10-2 }\end{array}$ & $\begin{array}{l}\text { CR- } \\
\text { CBM }\end{array}$ & 5 & 3 & 8.5 & 4.25 & 538.1 & 725 & 2 & 22.264 & $6.18 \mathrm{E}-03$ \\
\hline $\begin{array}{l}\text { CR Field } 1 \\
\text { DR 10-2 }\end{array}$ & $\begin{array}{l}\text { CR- } \\
\text { CBM }\end{array}$ & 7 & 4 & 10.5 & 5.25 & 664.7 & 725 & 2 & 27.503 & 7.64E-03 \\
\hline $\begin{array}{c}\text { CR Field } 1 \\
\text { DR 10-2 }\end{array}$ & $\begin{array}{l}\text { CR- } \\
\text { CBM }\end{array}$ & 9 & 5 & 9.1 & 4.55 & 576.0 & 725 & 2 & 23.836 & 6.62E-03 \\
\hline $\begin{array}{c}\text { CR Field } 1 \\
\text { DR 10-2 }\end{array}$ & $\begin{array}{l}\text { CR- } \\
\text { CBM }\end{array}$ & 15 & 7 & 26.9 & 4.48 & 1702.8 & 725 & 6 & 23.486 & $6.52 \mathrm{E}-03$ \\
\hline $\begin{array}{c}\text { CR Field } 1 \\
\text { DR 10-2 }\end{array}$ & $\begin{array}{l}\text { CR- } \\
\text { CBM }\end{array}$ & 20 & 8 & 20.2 & 4.04 & 1278.7 & 725 & 5 & 21.164 & $5.88 \mathrm{E}-03$ \\
\hline $\begin{array}{c}\text { CR Field } 1 \\
\text { DR 10-2 }\end{array}$ & $\begin{array}{l}\text { CR- } \\
\text { CBM }\end{array}$ & 23 & 10 & 8 & 2.67 & 506.4 & 725 & 3 & 13.970 & 3.88E-03 \\
\hline $\begin{array}{l}\text { CR Field } 1 \\
\text { DR 10-2 }\end{array}$ & $\begin{array}{l}\text { CR- } \\
\text { CBM }\end{array}$ & 25 & 11 & 7.7 & 3.85 & 487.4 & 725 & 2 & 20.169 & $5.60 \mathrm{E}-03$ \\
\hline $\begin{array}{c}\text { CR Field } 1 \\
\text { DR 10-2 }\end{array}$ & $\begin{array}{l}\text { CR- } \\
\text { CBM }\end{array}$ & 29 & 12 & 14.2 & 3.55 & 898.9 & 725 & 4 & 18.597 & 5.17E-03 \\
\hline $\begin{array}{c}\text { CR Field } 1 \\
\text { DR 10-2 }\end{array}$ & $\begin{array}{l}\text { CR- } \\
\text { CBM }\end{array}$ & 31 & 13 & 6.6 & 3.30 & 417.8 & 725 & 2 & 17.287 & $4.80 \mathrm{E}-03$ \\
\hline $\begin{array}{c}\text { CR Field } 1 \\
\text { DR } 10-2\end{array}$ & $\begin{array}{l}\text { CR- } \\
\text { CBM }\end{array}$ & 33 & 14 & 6.3 & 3.15 & 398.8 & 725 & 2 & 16.502 & $4.58 \mathrm{E}-03$ \\
\hline $\begin{array}{c}\text { CR Field } 1 \\
\text { DR 10-2 }\end{array}$ & $\begin{array}{l}\text { CR- } \\
\text { CBM }\end{array}$ & 36 & 16 & 6.6 & 2.20 & 417.8 & 725 & 3 & 11.525 & $3.20 \mathrm{E}-03$ \\
\hline $\begin{array}{c}\text { CR Field } 1 \\
\text { DR 10-2 }\end{array}$ & $\begin{array}{l}\text { CR- } \\
\text { CBM }\end{array}$ & 38 & 17 & 7 & 3.50 & 443.1 & 725 & 2 & 18.335 & 5.09E-03 \\
\hline $\begin{array}{l}\text { CR Field } 1 \\
\text { DR 10-2 }\end{array}$ & $\begin{array}{l}\text { CR- } \\
\text { CBM }\end{array}$ & 40 & 18 & 5.8 & 2.90 & 367.1 & 725 & 2 & 15.192 & $4.22 \mathrm{E}-03$ \\
\hline CR Field 1 & CR- & 42 & 19 & 6 & 3.00 & 379.8 & 725 & 2 & 15.716 & $4.37 \mathrm{E}-03$ \\
\hline
\end{tabular}




\begin{tabular}{|c|c|c|c|c|c|c|c|c|c|c|}
\hline DR $10-2$ & CBM & & & & & & & & & \\
\hline $\begin{array}{c}\text { CR Field } 1 \\
\text { DR 10-2 }\end{array}$ & $\begin{array}{l}\text { CR- } \\
\text { CBM }\end{array}$ & 44 & 20 & 5.3 & 2.65 & 335.5 & 725 & 2 & 13.882 & 3.86E-03 \\
\hline $\begin{array}{l}\text { CR Field } 1 \\
\text { DR } 10-2\end{array}$ & $\begin{array}{l}\text { CR- } \\
\text { CBM }\end{array}$ & 46 & 21 & 4.6 & 2.30 & 291.2 & 725 & 2 & 12.049 & 3.35E-03 \\
\hline $\begin{array}{c}\text { CR Field } 1 \\
\text { DR } 10-2\end{array}$ & $\begin{array}{l}\text { CR- } \\
\text { CBM }\end{array}$ & 48 & 22 & 5.8 & 2.90 & 367.1 & 725 & 2 & 15.192 & $4.22 \mathrm{E}-03$ \\
\hline $\begin{array}{c}\text { CR Field } 1 \\
\text { DR } 10-2\end{array}$ & $\begin{array}{l}\text { CR- } \\
\text { CBM }\end{array}$ & 51 & 24 & 5.8 & 1.93 & 367.1 & 725 & 3 & 10.128 & $2.81 \mathrm{E}-03$ \\
\hline $\begin{array}{c}\text { CR Field } 1 \\
\text { DR } 10-2\end{array}$ & $\begin{array}{l}\text { CR- } \\
\text { CBM }\end{array}$ & 53 & 25 & 4.8 & 2.40 & 303.8 & 725 & 2 & 12.573 & 3.49E-03 \\
\hline $\begin{array}{c}\text { CR Field } 1 \\
\text { DR } 10-2\end{array}$ & $\begin{array}{l}\text { CR- } \\
\text { CBM }\end{array}$ & 55 & 26 & 5.4 & 2.70 & 341.8 & 725 & 2 & 14.144 & 3.93E-03 \\
\hline $\begin{array}{c}\text { CR Field } 1 \\
\text { DR 10-2 }\end{array}$ & $\begin{array}{l}\text { CR- } \\
\text { CBM }\end{array}$ & 57 & 27 & 5.1 & 2.55 & 322.8 & 725 & 2 & 13.358 & 3.71E-03 \\
\hline $\begin{array}{c}\text { CR Field } 1 \\
\text { DR 10-2 }\end{array}$ & $\begin{array}{l}\text { CR- } \\
\text { CBM }\end{array}$ & 59 & 28 & 5.1 & 2.55 & 322.8 & 725 & 2 & 13.358 & $3.71 \mathrm{E}-03$ \\
\hline $\begin{array}{c}\text { CR Field } 1 \\
\text { DR } 10-2\end{array}$ & $\begin{array}{l}\text { CR- } \\
\text { CBM }\end{array}$ & 61 & 29 & 5 & 2.50 & 316.5 & 725 & 2 & 13.097 & 3.64E-03 \\
\hline & & & & & & & & $\begin{array}{r}\text { Final } \\
\text { Infiltration } \\
\text { Rate: }\end{array}$ & 13.27 & $3.69 \mathrm{E}-03$ \\
\hline & & & & & & & & & & \\
\hline & & & & & & & & & & \\
\hline \multicolumn{11}{|l|}{ 6/29/2003 } \\
\hline \multicolumn{11}{|l|}{ First Test } \\
\hline Test ID & $\begin{array}{l}\text { Water } \\
\text { Used }\end{array}$ & $\begin{array}{l}\text { Time } \\
\text { min }\end{array}$ & Reading & $\begin{array}{c}\text { Flow } \\
\text { Reading, cm } \\
\end{array}$ & $\begin{array}{c}\text { Flow, } \\
\mathrm{cm} / \mathrm{min}\end{array}$ & $\begin{array}{r}\begin{array}{l}V_{\text {IR }}, \\
\mathrm{cm}^{3}\end{array} \\
\end{array}$ & $\begin{array}{l}A_{\mathrm{IR}} \\
\mathrm{cm}^{2}\end{array}$ & $t, \min$ & $V_{\mathrm{IR}}, \mathrm{cm} / \mathrm{h}$ & $V_{\mathrm{IR}}, \mathrm{cm} / \mathrm{s}$ \\
\hline $\begin{array}{c}\text { CR Field } 1 \\
\text { DR 11-1 }\end{array}$ & $\begin{array}{l}\text { Piney } \\
\text { Creek }\end{array}$ & 1 & 1 & 9.60 & 9.60 & 607.7 & 730 & 1 & 49.946 & $1.39 \mathrm{E}-02$ \\
\hline $\begin{array}{c}\text { CR Field } 1 \\
\text { DR 11-1 }\end{array}$ & $\begin{array}{l}\text { Piney } \\
\text { Creek }\end{array}$ & 2 & 2 & 4.5 & 4.50 & 284.9 & 730 & 1 & 23.412 & $6.50 \mathrm{E}-03$ \\
\hline $\begin{array}{c}\text { CR Field } 1 \\
\text { DR 11-1 }\end{array}$ & $\begin{array}{l}\text { Piney } \\
\text { Creek }\end{array}$ & 3 & 3 & 4.3 & 4.30 & 272.2 & 730 & 1 & 22.372 & $6.21 \mathrm{E}-03$ \\
\hline $\begin{array}{c}\text { CR Field } 1 \\
\text { DR 11-1 }\end{array}$ & $\begin{array}{l}\text { Piney } \\
\text { Creek }\end{array}$ & 5 & 4 & 3.4 & 1.70 & 215.2 & 730 & 2 & 8.845 & $2.46 \mathrm{E}-03$ \\
\hline $\begin{array}{c}\text { CR Field } 1 \\
\text { DR 11-1 }\end{array}$ & $\begin{array}{l}\text { Piney } \\
\text { Creek }\end{array}$ & 7 & 5 & 3.3 & 1.65 & 208.9 & 730 & 2 & 8.585 & $2.38 \mathrm{E}-03$ \\
\hline $\begin{array}{c}\text { CR Field } 1 \\
\text { DR 11-1 }\end{array}$ & $\begin{array}{l}\text { Piney } \\
\text { Creek }\end{array}$ & 11 & 6 & 7 & 1.75 & 443.1 & 730 & 4 & 9.105 & 2.53E-03 \\
\hline $\begin{array}{c}\text { CR Field } 1 \\
\text { DR 11-1 }\end{array}$ & $\begin{array}{l}\text { Piney } \\
\text { Creek }\end{array}$ & 15 & 7 & 6.5 & 1.63 & 411.5 & 730 & 4 & 8.454 & $2.35 \mathrm{E}-03$ \\
\hline $\begin{array}{c}\text { CR Field } 1 \\
\text { DR 11-1 }\end{array}$ & $\begin{array}{l}\text { Piney } \\
\text { Creek }\end{array}$ & 19 & 8 & 5.8 & 1.45 & 367.1 & 730 & 4 & 7.544 & $2.10 \mathrm{E}-03$ \\
\hline $\begin{array}{c}\text { CR Field } 1 \\
\text { DR 11-1 }\end{array}$ & $\begin{array}{l}\text { Piney } \\
\text { Creek }\end{array}$ & 24 & 10 & 6.4 & 1.28 & 405.1 & 730 & 5 & 6.660 & $1.85 \mathrm{E}-03$ \\
\hline $\begin{array}{c}\text { CR Field } 1 \\
\text { DR 11-1 }\end{array}$ & $\begin{array}{l}\text { Piney } \\
\text { Creek }\end{array}$ & 28 & 11 & 5 & 1.25 & 316.5 & 730 & 4 & 6.503 & $1.81 \mathrm{E}-03$ \\
\hline $\begin{array}{c}\text { CR Field } 1 \\
\text { DR 11-1 }\end{array}$ & $\begin{array}{l}\text { Piney } \\
\text { Creek }\end{array}$ & 32 & 12 & 4.4 & 1.10 & 278.5 & 730 & 4 & 5.723 & 1.59E-03 \\
\hline $\begin{array}{c}\text { CR Field } 1 \\
\text { DR 11-1 }\end{array}$ & $\begin{array}{l}\text { Piney } \\
\text { Creek }\end{array}$ & 40 & 13 & 7.5 & 0.94 & 474.8 & 730 & 8 & 4.878 & $1.35 \mathrm{E}-03$ \\
\hline $\begin{array}{c}\text { CR Field } 1 \\
\text { DR 11-1 }\end{array}$ & $\begin{array}{l}\text { Piney } \\
\text { Creek }\end{array}$ & 44 & 14 & 3.5 & 0.88 & 221.6 & 730 & 4 & 4.552 & $1.26 \mathrm{E}-03$ \\
\hline $\begin{array}{c}\text { CR Field } 1 \\
\text { DR 11-1 }\end{array}$ & $\begin{array}{l}\text { Piney } \\
\text { Creek }\end{array}$ & 48 & 15 & 0.3 & 0.08 & 19.0 & 730 & 4 & 0.390 & $1.08 \mathrm{E}-04$ \\
\hline
\end{tabular}




\begin{tabular}{|c|c|c|c|c|c|c|c|c|c|c|}
\hline $\begin{array}{c}\text { CR Field } 1 \\
\text { DR 11-1 }\end{array}$ & $\begin{array}{l}\text { Piney } \\
\text { Creek }\end{array}$ & 52 & 16 & 2.6 & 0.65 & 164.6 & 730 & 4 & 3.382 & $9.39 \mathrm{E}-04$ \\
\hline $\begin{array}{c}\text { CR Field } 1 \\
\text { DR 11-1 }\end{array}$ & $\begin{array}{l}\text { Piney } \\
\text { Creek }\end{array}$ & 56 & 17 & 3 & 0.75 & 189.9 & 730 & 4 & 3.902 & $1.08 \mathrm{E}-03$ \\
\hline $\begin{array}{c}\text { CR Field } 1 \\
\text { DR 11-1 }\end{array}$ & $\begin{array}{l}\text { Piney } \\
\text { Creek }\end{array}$ & 60 & 18 & 3 & 0.75 & 189.9 & 730 & 4 & 3.902 & $1.08 \mathrm{E}-03$ \\
\hline $\begin{array}{c}\text { CR Field } 1 \\
\text { DR 11-1 }\end{array}$ & $\begin{array}{l}\text { Piney } \\
\text { Creek }\end{array}$ & 64 & 19 & 2.5 & 0.63 & 158.3 & 730 & 4 & 3.252 & 9.03E-04 \\
\hline $\begin{array}{c}\text { CR Field } 1 \\
\text { DR 11-1 }\end{array}$ & $\begin{array}{l}\text { Piney } \\
\text { Creek }\end{array}$ & 68 & 20 & 2.5 & 0.63 & 158.3 & 730 & 4 & 3.252 & 9.03E-04 \\
\hline $\begin{array}{c}\text { CR Field } 1 \\
\text { DR 11-1 }\end{array}$ & $\begin{array}{l}\text { Piney } \\
\text { Creek }\end{array}$ & 72 & 21 & 2.6 & 0.65 & 164.6 & 730 & 4 & 3.382 & $9.39 \mathrm{E}-04$ \\
\hline & & & & & & & & $\begin{array}{r}\text { Final } \\
\text { Infiltration } \\
\text { Rate: }\end{array}$ & 3.30 & 9.15E-04 \\
\hline & & & & & & & & & & \\
\hline & & & & & & & & & & \\
\hline 6/30/2003 & & & & & & & & & & \\
\hline $\begin{array}{l}\text { 2nd Test- } \\
\text { same } \\
\text { location }\end{array}$ & & & & & & & & & & \\
\hline Test ID & $\begin{array}{l}\text { Water } \\
\text { Used }\end{array}$ & $\begin{array}{l}\text { Time } \\
\text { min }\end{array}$ & Reading & $\begin{array}{c}\text { Flow } \\
\text { Reading, cm }\end{array}$ & $\begin{array}{l}\text { Flow, } \\
\mathrm{cm} / \mathrm{min}\end{array}$ & $\begin{array}{l}\begin{array}{l}V_{\text {IR }}, \\
\mathrm{cm}^{3}\end{array}\end{array}$ & $\begin{array}{l}A_{\mathrm{IR}} \\
\mathrm{cm}^{2}\end{array}$ & $t, \min$ & $V_{\mathrm{IR}}, \mathrm{cm} / \mathrm{h}$ & $V_{\mathrm{IR}}, \mathrm{cm} / \mathrm{s}$ \\
\hline $\begin{array}{c}\text { CR Field } 1 \\
\text { DR } 11-2\end{array}$ & $\begin{array}{l}\text { CR- } \\
\text { CBM }\end{array}$ & 2 & 1 & 0.60 & 0.30 & 38.0 & 725 & 2 & 1.572 & 4.37E-04 \\
\hline $\begin{array}{c}\text { CR Field } 1 \\
\text { DR } 11-2\end{array}$ & $\begin{array}{l}\text { CR- } \\
\text { CBM }\end{array}$ & 4 & 2 & 1.5 & 0.75 & 95.0 & 725 & 2 & 3.929 & $1.09 \mathrm{E}-03$ \\
\hline $\begin{array}{c}\text { CR Field } 1 \\
\text { DR 11-2 }\end{array}$ & $\begin{array}{l}\text { CR- } \\
\text { CBM }\end{array}$ & 6 & 3 & 1.3 & 0.65 & 82.3 & 725 & 2 & 3.405 & $9.46 \mathrm{E}-04$ \\
\hline $\begin{array}{c}\text { CR Field } 1 \\
\text { DR 11-2 }\end{array}$ & $\begin{array}{l}\text { CR- } \\
\text { CBM }\end{array}$ & 8 & 4 & 0.9 & 0.45 & 57.0 & 725 & 2 & 2.357 & $6.55 \mathrm{E}-04$ \\
\hline $\begin{array}{c}\text { CR Field } 1 \\
\text { DR 11-2 }\end{array}$ & $\begin{array}{l}\text { CR- } \\
\text { CBM }\end{array}$ & 10 & 5 & 1.2 & 0.60 & 76.0 & 725 & 2 & 3.143 & 8.73E-04 \\
\hline $\begin{array}{c}\text { CR Field } 1 \\
\text { DR 11-2 }\end{array}$ & $\begin{array}{l}\text { CR- } \\
\text { CBM }\end{array}$ & 15 & 6 & 2.1 & 0.42 & 132.9 & 725 & 5 & 2.200 & $6.11 \mathrm{E}-04$ \\
\hline $\begin{array}{c}\text { CR Field } 1 \\
\text { DR 11-2 }\end{array}$ & $\begin{array}{l}\text { CR- } \\
\text { CBM }\end{array}$ & 20 & 7 & 0.7 & 0.14 & 44.3 & 725 & 5 & 0.733 & $2.04 \mathrm{E}-04$ \\
\hline $\begin{array}{c}\text { CR Field } 1 \\
\text { DR } 11-2\end{array}$ & $\begin{array}{l}\text { CR- } \\
\text { CBM }\end{array}$ & 25 & 8 & 0.6 & 0.12 & 38.0 & 725 & 5 & 0.629 & $1.75 \mathrm{E}-04$ \\
\hline $\begin{array}{c}\text { CR Field } 1 \\
\text { DR 11-2 }\end{array}$ & $\begin{array}{l}\text { CR- } \\
\text { CBM }\end{array}$ & 30 & 9 & 0.5 & 0.10 & 31.7 & 725 & 5 & 0.524 & $1.46 \mathrm{E}-04$ \\
\hline $\begin{array}{c}\text { CR Field } 1 \\
\text { DR 11-2 }\end{array}$ & $\begin{array}{l}\text { CR- } \\
\text { CBM }\end{array}$ & 35 & 10 & 0.5 & 0.10 & 31.7 & 725 & 5 & 0.524 & $1.46 \mathrm{E}-04$ \\
\hline $\begin{array}{c}\text { CR Field } 1 \\
\text { DR 11-2 }\end{array}$ & $\begin{array}{l}\text { CR- } \\
\text { CBM }\end{array}$ & 40 & 11 & 0.2 & 0.04 & 12.7 & 725 & 5 & 0.210 & 5.82E-05 \\
\hline $\begin{array}{c}\text { CR Field } 1 \\
\text { DR 11-2 }\end{array}$ & $\begin{array}{l}\text { CR- } \\
\text { CBM }\end{array}$ & 45 & 12 & 1 & 0.20 & 63.3 & 725 & 5 & 1.048 & 2.91E-04 \\
\hline $\begin{array}{c}\text { CR Field } 1 \\
\text { DR 11-2 }\end{array}$ & $\begin{array}{l}\text { CR- } \\
\text { CBM }\end{array}$ & 50 & 13 & 0.8 & 0.16 & 50.6 & 725 & 5 & 0.838 & 2.33E-04 \\
\hline $\begin{array}{c}\text { CR Field } 1 \\
\text { DR 11-2 }\end{array}$ & $\begin{array}{l}\text { CR- } \\
\text { CBM }\end{array}$ & 55 & 14 & 1.4 & 0.28 & 88.6 & 725 & 5 & 1.467 & 4.07E-04 \\
\hline $\begin{array}{c}\text { CR Field } 1 \\
\text { DR 11-2 }\end{array}$ & $\begin{array}{l}\text { CR- } \\
\text { CBM }\end{array}$ & 60 & 15 & 1.4 & 0.28 & 88.6 & 725 & 5 & 1.467 & 4.07E-04 \\
\hline $\begin{array}{c}\text { CR Field } 1 \\
\text { DR 11-2 }\end{array}$ & $\begin{array}{l}\text { CR- } \\
\text { CBM }\end{array}$ & 65 & 16 & 1.5 & 0.30 & 95.0 & 725 & 5 & 1.572 & 4.37E-04 \\
\hline CR Field 1 & CR- & 70 & 17 & 1.4 & 0.28 & 88.6 & 725 & 5 & 1.467 & $4.07 \mathrm{E}-04$ \\
\hline
\end{tabular}




\begin{tabular}{|c|c|c|c|c|c|c|c|c|c|c|}
\hline \multirow[t]{2}{*}{ DR 11-2 } & \multirow[t]{2}{*}{ CBM } & & & & & & & \multirow[b]{2}{*}{$\begin{array}{r}\text { Final } \\
\text { Infiltration } \\
\text { Rate: }\end{array}$} & \multirow[b]{2}{*}{1.49} & \multirow[b]{2}{*}{ 4.15E-04 } \\
\hline & & & & & & & & & & \\
\hline & & & & & & & & & & \\
\hline & & & & & & & & & & \\
\hline & & & & & & & & & & \\
\hline \multicolumn{11}{|l|}{$6 / 29 / 2003$} \\
\hline \multicolumn{11}{|l|}{ First Test } \\
\hline Test ID & $\begin{array}{l}\text { Water } \\
\text { Used }\end{array}$ & $\begin{array}{c}\text { Time } \\
\text { min }\end{array}$ & Reading & $\begin{array}{c}\text { Flow } \\
\text { Reading, cm }\end{array}$ & $\begin{array}{l}\text { Flow, } \\
\mathrm{cm} / \mathrm{min}\end{array}$ & $\begin{array}{r}\begin{array}{l}V_{\mathrm{IR}}, \\
\mathrm{cm}^{3}\end{array} \\
\text {, }\end{array}$ & $\begin{array}{l}A_{\mathrm{IR}}, \\
\mathrm{cm}^{2}\end{array}$ & $t, \min$ & $V_{\mathrm{IR}}, \mathrm{cm} / \mathrm{h}$ & $V_{\mathrm{IR}}, \mathrm{cm} / \mathrm{s}$ \\
\hline $\begin{array}{l}\text { CR Field } 1 \\
\text { DR 12-1 }\end{array}$ & $\begin{array}{l}\text { Piney } \\
\text { Creek }\end{array}$ & 1 & 1 & 13.10 & 13.10 & 829.2 & 730 & 1 & 68.156 & 1.89E-02 \\
\hline $\begin{array}{c}\text { CR Field } 1 \\
\text { DR } 12-1\end{array}$ & $\begin{array}{l}\text { Piney } \\
\text { Creek }\end{array}$ & 2 & 2 & 5.4 & 5.40 & 341.8 & 730 & 1 & 28.095 & $7.80 \mathrm{E}-03$ \\
\hline $\begin{array}{c}\text { CR Field } 1 \\
\text { DR 12-1 }\end{array}$ & $\begin{array}{l}\text { Piney } \\
\text { Creek }\end{array}$ & 3 & 3 & 1.7 & 1.70 & 107.6 & 730 & 1 & 8.845 & $2.46 \mathrm{E}-03$ \\
\hline $\begin{array}{c}\text { CR Field } 1 \\
\text { DR 12-1 }\end{array}$ & $\begin{array}{l}\text { Piney } \\
\text { Creek }\end{array}$ & 5 & 4 & 4.6 & 2.30 & 291.2 & 730 & 2 & 11.966 & 3.32E-03 \\
\hline $\begin{array}{c}\text { CR Field } 1 \\
\text { DR 12-1 }\end{array}$ & $\begin{array}{l}\text { Piney } \\
\text { Creek }\end{array}$ & 7 & 5 & 4.7 & 2.35 & 297.5 & 730 & 2 & 12.226 & $3.40 \mathrm{E}-03$ \\
\hline $\begin{array}{c}\text { CR Field } 1 \\
\text { DR 12-1 }\end{array}$ & $\begin{array}{l}\text { Piney } \\
\text { Creek }\end{array}$ & 11 & 6 & 8.2 & 2.05 & 519.1 & 730 & 4 & 10.666 & $2.96 \mathrm{E}-03$ \\
\hline $\begin{array}{c}\text { CR Field } 1 \\
\text { DR } 12-1\end{array}$ & $\begin{array}{l}\text { Piney } \\
\text { Creek }\end{array}$ & 15 & 7 & 7.1 & 1.78 & 449.4 & 730 & 4 & 9.235 & $2.57 \mathrm{E}-03$ \\
\hline $\begin{array}{c}\text { CR Field } 1 \\
\text { DR 12-1 }\end{array}$ & $\begin{array}{l}\text { Piney } \\
\text { Creek }\end{array}$ & 20 & 9 & 5.9 & 1.18 & 373.5 & 730 & 5 & 6.139 & $1.71 \mathrm{E}-03$ \\
\hline $\begin{array}{c}\text { CR Field } 1 \\
\text { DR 12-1 }\end{array}$ & $\begin{array}{l}\text { Piney } \\
\text { Creek }\end{array}$ & 24 & 10 & 4 & 1.00 & 253.2 & 730 & 4 & 5.203 & $1.45 \mathrm{E}-03$ \\
\hline $\begin{array}{c}\text { CR Field } 1 \\
\text { DR 12-1 }\end{array}$ & $\begin{array}{l}\text { Piney } \\
\text { Creek }\end{array}$ & 28 & 11 & 2.8 & 0.70 & 177.2 & 730 & 4 & 3.642 & $1.01 \mathrm{E}-03$ \\
\hline $\begin{array}{c}\text { CR Field } 1 \\
\text { DR } 12-1\end{array}$ & $\begin{array}{l}\text { Piney } \\
\text { Creek }\end{array}$ & 32 & 12 & 2.1 & 0.53 & 132.9 & 730 & 4 & 2.731 & 7.59E-04 \\
\hline $\begin{array}{c}\text { CR Field } 1 \\
\text { DR 12-1 }\end{array}$ & $\begin{array}{l}\text { Piney } \\
\text { Creek }\end{array}$ & 36 & 13 & 2.4 & 0.60 & 151.9 & 730 & 4 & 3.122 & 8.67E-04 \\
\hline $\begin{array}{c}\text { CR Field } 1 \\
\text { DR 12-1 }\end{array}$ & $\begin{array}{l}\text { Piney } \\
\text { Creek }\end{array}$ & 40 & 14 & 2.3 & 0.58 & 145.6 & 730 & 4 & 2.992 & 8.31E-04 \\
\hline $\begin{array}{c}\text { CR Field } 1 \\
\text { DR 12-1 }\end{array}$ & $\begin{array}{l}\text { Piney } \\
\text { Creek }\end{array}$ & 44 & 15 & 2.2 & 0.55 & 139.3 & 730 & 4 & 2.862 & 7.95E-04 \\
\hline $\begin{array}{c}\text { CR Field } 1 \\
\text { DR 12-1 }\end{array}$ & $\begin{array}{l}\text { Piney } \\
\text { Creek }\end{array}$ & 48 & 16 & 2.5 & 0.63 & 158.3 & 730 & 4 & 3.252 & $9.03 \mathrm{E}-04$ \\
\hline $\begin{array}{c}\text { CR Field } 1 \\
\text { DR 12-1 }\end{array}$ & $\begin{array}{l}\text { Piney } \\
\text { Creek }\end{array}$ & 52 & 17 & 2.3 & 0.58 & 145.6 & 730 & 4 & 2.992 & 8.31E-04 \\
\hline $\begin{array}{c}\text { CR Field } 1 \\
\text { DR } 12-1\end{array}$ & $\begin{array}{l}\text { Piney } \\
\text { Creek }\end{array}$ & 56 & 18 & 2.2 & 0.55 & 139.3 & 730 & 4 & 2.862 & 7.95E-04 \\
\hline $\begin{array}{c}\text { CR Field } 1 \\
\text { DR } 12-1\end{array}$ & $\begin{array}{l}\text { Piney } \\
\text { Creek }\end{array}$ & 60 & 19 & 2.5 & 0.63 & 158.3 & 730 & 4 & 3.252 & $9.03 \mathrm{E}-04$ \\
\hline $\begin{array}{c}\text { CR Field } 1 \\
\text { DR 12-1 }\end{array}$ & $\begin{array}{l}\text { Piney } \\
\text { Creek }\end{array}$ & 64 & 20 & 2.3 & 0.58 & 145.6 & 730 & 4 & 2.992 & 8.31E-04 \\
\hline $\begin{array}{c}\text { CR Field } 1 \\
\text { DR } 12-1\end{array}$ & $\begin{array}{l}\text { Piney } \\
\text { Creek }\end{array}$ & 68 & 21 & 2.7 & 0.68 & 170.9 & 730 & 4 & 3.512 & $9.76 \mathrm{E}-04$ \\
\hline $\begin{array}{c}\text { CR Field } 1 \\
\text { DR 12-1 }\end{array}$ & $\begin{array}{l}\text { Piney } \\
\text { Creek }\end{array}$ & 72 & 22 & 1.6 & 0.40 & 101.3 & 730 & 4 & 2.081 & $5.78 \mathrm{E}-04$ \\
\hline $\begin{array}{c}\text { CR Field } 1 \\
\text { DR 12-1 }\end{array}$ & $\begin{array}{l}\text { Piney } \\
\text { Creek }\end{array}$ & 76 & 23 & 2.4 & 0.60 & 151.9 & 730 & 4 & 3.122 & 8.67E-04 \\
\hline
\end{tabular}




\begin{tabular}{|c|c|c|c|c|c|c|c|c|c|c|}
\hline $\begin{array}{c}\text { CR Field } 1 \\
\text { DR 12-1 }\end{array}$ & $\begin{array}{l}\text { Piney } \\
\text { Creek }\end{array}$ & 80 & 24 & 2.2 & 0.55 & 139.3 & 730 & 4 & 2.862 & 7.95E-04 \\
\hline $\begin{array}{c}\text { CR Field } 1 \\
\text { DR 12-1 }\end{array}$ & $\begin{array}{l}\text { Piney } \\
\text { Creek }\end{array}$ & 84 & 25 & 2.2 & 0.55 & 139.3 & 730 & 4 & 2.862 & 7.95E-04 \\
\hline & & & & & & & & $\begin{array}{r}\text { Final } \\
\text { Infiltration } \\
\text { Rate: }\end{array}$ & 2.86 & 7.95E-04 \\
\hline & & & & & & & & & & \\
\hline & & & & & & & & & & \\
\hline 6/30/2003 & & & & & & & & & & \\
\hline $\begin{array}{l}\text { 2nd Test- } \\
\text { same } \\
\text { location }\end{array}$ & & & & & & & & & & \\
\hline Test ID & $\begin{array}{l}\text { Water } \\
\text { Used }\end{array}$ & $\begin{array}{l}\text { Time } \\
\text { min }\end{array}$ & Reading & $\begin{array}{c}\text { Flow } \\
\text { Reading, cm }\end{array}$ & $\begin{array}{l}\text { Flow, } \\
\mathrm{cm} / \mathrm{min}\end{array}$ & 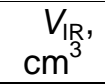 & $\begin{array}{l}A_{\mathrm{IR}} \\
\mathrm{cm}^{2}\end{array}$ & $t, \min$ & $V_{\mathrm{IR}}, \mathrm{cm} / \mathrm{h}$ & $V_{\mathrm{IR}}, \mathrm{cm} / \mathrm{s}$ \\
\hline $\begin{array}{c}\text { CR Field } 1 \\
\text { DR } 11-2\end{array}$ & $\begin{array}{l}\text { Piney } \\
\text { Creek }\end{array}$ & 2 & 1 & 0.00 & 0.00 & 0.0 & 725 & 2 & 0.000 & $0.00 \mathrm{E}+00$ \\
\hline $\begin{array}{c}\text { CR Field } 1 \\
\text { DR 11-2 }\end{array}$ & $\begin{array}{l}\text { Piney } \\
\text { Creek }\end{array}$ & 4 & 2 & 0 & 0.00 & 0.0 & 725 & 2 & 0.000 & $0.00 \mathrm{E}+00$ \\
\hline $\begin{array}{c}\text { CR Field } 1 \\
\text { DR 11-2 }\end{array}$ & $\begin{array}{l}\text { Piney } \\
\text { Creek }\end{array}$ & 6 & 3 & 0 & 0.00 & 0.0 & 725 & 2 & 0.000 & $0.00 \mathrm{E}+00$ \\
\hline $\begin{array}{c}\text { CR Field } 1 \\
\text { DR 11-2 }\end{array}$ & $\begin{array}{l}\text { Piney } \\
\text { Creek }\end{array}$ & 8 & 4 & 0.2 & 0.10 & 12.7 & 725 & 2 & 0.524 & $1.46 \mathrm{E}-04$ \\
\hline $\begin{array}{c}\text { CR Field } 1 \\
\text { DR 11-2 }\end{array}$ & $\begin{array}{l}\text { Piney } \\
\text { Creek }\end{array}$ & 10 & 5 & 0.3 & 0.15 & 19.0 & 725 & 2 & 0.786 & $2.18 \mathrm{E}-04$ \\
\hline $\begin{array}{c}\text { CR Field } 1 \\
\text { DR 11-2 }\end{array}$ & $\begin{array}{l}\text { Piney } \\
\text { Creek }\end{array}$ & 15 & 6 & 1.7 & 0.34 & 107.6 & 725 & 5 & 1.781 & 4.95E-04 \\
\hline $\begin{array}{c}\text { CR Field } 1 \\
\text { DR 11-2 }\end{array}$ & $\begin{array}{l}\text { Piney } \\
\text { Creek }\end{array}$ & 20 & 7 & 3 & 0.60 & 189.9 & 725 & 5 & 3.143 & 8.73E-04 \\
\hline $\begin{array}{c}\text { CR Field } 1 \\
\text { DR 11-2 }\end{array}$ & $\begin{array}{l}\text { Piney } \\
\text { Creek }\end{array}$ & 25 & 8 & 2.4 & 0.48 & 151.9 & 725 & 5 & 2.515 & $6.98 \mathrm{E}-04$ \\
\hline $\begin{array}{c}\text { CR Field } 1 \\
\text { DR 11-2 }\end{array}$ & $\begin{array}{l}\text { Piney } \\
\text { Creek }\end{array}$ & 30 & 9 & 3.8 & 0.76 & 240.5 & 725 & 5 & 3.981 & $1.11 \mathrm{E}-03$ \\
\hline $\begin{array}{c}\text { CR Field } 1 \\
\text { DR } 11-2\end{array}$ & $\begin{array}{l}\text { Piney } \\
\text { Creek }\end{array}$ & 35 & 10 & 4.3 & 0.86 & 272.2 & 725 & 5 & 4.505 & $1.25 \mathrm{E}-03$ \\
\hline $\begin{array}{c}\text { CR Field } 1 \\
\text { DR 11-2 }\end{array}$ & $\begin{array}{l}\text { Piney } \\
\text { Creek }\end{array}$ & 40 & 11 & 3.5 & 0.70 & 221.6 & 725 & 5 & 3.667 & $1.02 \mathrm{E}-03$ \\
\hline $\begin{array}{c}\text { CR Field } 1 \\
\text { DR 11-2 }\end{array}$ & $\begin{array}{l}\text { Piney } \\
\text { Creek }\end{array}$ & 45 & 12 & 3 & 0.60 & 189.9 & 725 & 5 & 3.143 & 8.73E-04 \\
\hline $\begin{array}{c}\text { CR Field } 1 \\
\text { DR 11-2 }\end{array}$ & $\begin{array}{l}\text { Piney } \\
\text { Creek }\end{array}$ & 50 & 13 & 2.5 & 0.50 & 158.3 & 725 & 5 & 2.619 & $7.28 \mathrm{E}-04$ \\
\hline $\begin{array}{c}\text { CR Field } 1 \\
\text { DR 11-2 }\end{array}$ & $\begin{array}{l}\text { Piney } \\
\text { Creek }\end{array}$ & 55 & 14 & 2.4 & 0.48 & 151.9 & 725 & 5 & 2.515 & $6.98 \mathrm{E}-04$ \\
\hline $\begin{array}{c}\text { CR Field } 1 \\
\text { DR 11-2 }\end{array}$ & $\begin{array}{l}\text { Piney } \\
\text { Creek }\end{array}$ & 60 & 15 & 2.2 & 0.44 & 139.3 & 725 & 5 & 2.305 & $6.40 \mathrm{E}-04$ \\
\hline $\begin{array}{c}\text { CR Field } 1 \\
\text { DR 11-2 }\end{array}$ & $\begin{array}{l}\text { Piney } \\
\text { Creek }\end{array}$ & 65 & 16 & 2.2 & 0.44 & 139.3 & 725 & 5 & 2.305 & $6.40 \mathrm{E}-04$ \\
\hline $\begin{array}{c}\text { CR Field } 1 \\
\text { DR 11-2 }\end{array}$ & $\begin{array}{l}\text { Piney } \\
\text { Creek }\end{array}$ & 70 & 17 & 1.9 & 0.38 & 120.3 & 725 & 5 & 1.991 & $5.53 \mathrm{E}-04$ \\
\hline $\begin{array}{c}\text { CR Field } 1 \\
\text { DR } 11-2\end{array}$ & $\begin{array}{l}\text { Piney } \\
\text { Creek }\end{array}$ & 75 & 18 & 2 & 0.40 & 126.6 & 725 & 5 & 2.095 & $5.82 \mathrm{E}-04$ \\
\hline \multirow[t]{2}{*}{$\begin{array}{c}\text { CR Field } 1 \\
\text { DR 11-2 }\end{array}$} & $\begin{array}{l}\text { Piney } \\
\text { Creek }\end{array}$ & 80 & 19 & 2 & 0.40 & 126.6 & 725 & 5 & 2.095 & $5.82 \mathrm{E}-04$ \\
\hline & & & & & & & & Final & 2.10 & $5.82 \mathrm{E}-04$ \\
\hline
\end{tabular}




\section{Appendix V}

Baseline Soil Chemistry Data from Cooksley Ranch Site - Fall 2003. 
Irrigation

\begin{tabular}{|c|c|c|c|c|c|c|c|c|c|c|c|c|c|}
\hline Plot & Treatment & $\begin{array}{c}\text { Plot } \\
\text { Treatment }\end{array}$ & $\begin{array}{c}\text { Sample } \\
\text { Depth }\end{array}$ & $\begin{array}{c}\text { Soil } \\
\text { Horizon }\end{array}$ & $\begin{array}{l}\mathrm{pH} \\
\text { s.u. }\end{array}$ & $\begin{array}{c}\mathrm{EC} \\
\mathrm{mmhos} / \mathrm{cm}\end{array}$ & $\begin{array}{c}\mathrm{Na} \\
\mathrm{meq} / \mathrm{L}\end{array}$ & $\begin{array}{c}\mathrm{Ca} \\
\mathrm{meq} / \mathrm{L}\end{array}$ & $\begin{array}{c}\mathrm{Mg} \\
\mathrm{meq} / \mathrm{L}\end{array}$ & SAR & $\begin{array}{c}\text { Alkalinity } \\
\mathrm{mg} / \mathrm{L}\end{array}$ & $\begin{array}{c}\mathrm{Cl} \\
\mathrm{meq} / \mathrm{L}\end{array}$ & $\begin{array}{c}\mathrm{SO} 4 \\
\mathrm{meq} / \mathrm{L}\end{array}$ \\
\hline $1-1$ & PC & GS & $0-4.5$ & $\mathrm{~A}$ & 7.1 & 0.9 & 1.1 & 6.8 & 2.9 & 0.5 & 10 & 0.3 & 0.2 \\
\hline $1-1$ & PC & GS & 4.5-12 & Bt1 & 7.4 & 0.6 & 1.3 & 4.2 & 1.8 & 0.7 & 4.1 & 0.3 & 0.4 \\
\hline $1-1$ & PC & GS & $12-24$ & Bt2 & 7.7 & 0.5 & 1.2 & 3.2 & 1.2 & 0.8 & 3.4 & 0.3 & 0.4 \\
\hline $1-2$ & PC & $\mathrm{G}$ & $0-4.75$ & $\mathrm{~A}$ & 7.2 & 0.8 & 1 & 5.1 & 2.3 & 0.5 & 6.7 & 0.3 & 0.2 \\
\hline $1-2$ & PC & G & $4.75-13.5$ & Bt1 & 7.5 & 0.6 & 1.3 & 3.7 & 1.7 & 0.8 & 3.5 & 0.3 & 0.4 \\
\hline $1-2$ & PC & $\mathrm{G}$ & $13.5-24$ & Bt2 & 8.2 & 0.43 & 1.1 & 2.4 & 0.9 & 0.8 & 7.4 & 0.3 & 0.6 \\
\hline $1-3$ & $\mathrm{PC}$ & NT & $0-6$ & A & 7.2 & 0.8 & 1.2 & 6.2 & 2.4 & 0.6 & 2.5 & 0.3 & 0.2 \\
\hline $1-3$ & PC & NT & $6-13.5$ & Bt1 & 7.8 & 0.48 & 1.1 & 2.7 & 1 & 0.8 & 3.2 & 0.3 & 0.5 \\
\hline $1-3$ & PC & NT & $13.5-24$ & Bt2 & 8 & 0.44 & 1.1 & 2.4 & 0.8 & 0.9 & 2.4 & 0.3 & 0.7 \\
\hline $1-4$ & PC & $\mathrm{S}$ & $0-6$ & $\mathrm{~A}$ & 7.5 & 0.91 & 0.9 & 5.2 & 1.9 & 0.5 & 6.4 & 0.3 & 0.7 \\
\hline $1-4$ & PC & S & 6-13.5 & Bt1 & 7.9 & 0.52 & 1.3 & 2.8 & 1 & 0.9 & 2.8 & 0.3 & 0.8 \\
\hline $1-4$ & PC & $S$ & $13.5-24$ & $\mathrm{Bt} 2$ & 8 & 0.52 & 1.3 & 2.8 & 1 & 0.9 & 2.8 & 0.3 & 0.9 \\
\hline $2-1$ & PC & G & $0-4.5$ & A & 7.5 & 0.92 & 0.9 & 6.2 & 2.3 & 0.4 & 7.2 & 0.3 & 0.5 \\
\hline $2-1$ & PC & $\mathrm{G}$ & $4.5-12.75$ & Bt1 & 7.7 & 0.6 & 1.2 & 3.1 & 1.3 & 0.8 & 3.6 & 0.3 & 0.8 \\
\hline $2-1$ & PC & $\mathrm{G}$ & $12.75-24$ & Bt2 & 7.9 & 0.53 & 1.1 & 2.7 & 1.1 & 0.8 & 2.6 & 0.3 & 1.1 \\
\hline $2-2$ & PC & NT & $0-5$ & A & 7.5 & 0.71 & 0.7 & 4.5 & 1.6 & 0.4 & 6 & 0.3 & 0.3 \\
\hline $2-2$ & PC & NT & 5-14.5 & Bt1 & 7.8 & 0.39 & 1.1 & 2.2 & 0.8 & 0.9 & 3 & 0.3 & 0.4 \\
\hline $2-2$ & PC & NT & $14.5-24$ & Bt2 & 7.9 & 0.41 & 1 & 2.4 & 0.9 & 0.8 & 2.7 & 0.3 & 0.7 \\
\hline $2-3$ & PC & $\mathrm{S}$ & $0-5.5$ & $\mathrm{~A}$ & 7.5 & 0.67 & 0.9 & 2.4 & 0.8 & 0.7 & 4.9 & 0.5 & 0.7 \\
\hline $2-3$ & PC & S & 5.5-13 & Bt1 & 7.7 & 0.61 & 1.2 & 3.6 & 1.4 & 0.8 & 3.6 & 0.4 & 1 \\
\hline $2-3$ & PC & $\mathrm{S}$ & $13-24$ & Bt2 & 7.9 & 0.63 & 1.3 & 3.5 & 1.4 & 0.8 & 3.5 & 0.3 & 1.6 \\
\hline $2-4$ & PC & GS & $0-6$ & A & 7.5 & 0.66 & 0.8 & 4.1 & 1.4 & 0.5 & 6.5 & 0.4 & 0.5 \\
\hline $2-4$ & PC & GS & 6-13.5 & Bt1 & 7.8 & 0.44 & 0.9 & 2.7 & 1 & 0.7 & 4.2 & 0.4 & 0.6 \\
\hline
\end{tabular}




\begin{tabular}{|c|c|c|c|c|c|c|c|c|c|c|c|c|c|}
\hline $3-1$ & CBM & NT & $0-5$ & A & 7.6 & 0.56 & 1 & 3.2 & 1.1 & 0.9 & 6.7 & 1 & 0.3 \\
\hline 3-1 & CBM & NT & $5-14$ & Bt1 & 7.7 & 0.44 & 1.1 & 2.4 & 0.8 & 0.9 & 2.8 & 0.4 & 0.6 \\
\hline $3-1$ & CBM & NT & $14-24$ & $\mathrm{Bt} 2$ & 7.8 & 0.46 & 1.1 & 2.6 & 0.8 & 0.9 & 2.6 & 0.4 & 0.7 \\
\hline $3-2$ & CBM & GS & $0-5$ & $\mathrm{~A}$ & 7.5 & 0.81 & 0.9 & 5.2 & 2 & 0.5 & 5.9 & 0.5 & 0.7 \\
\hline $3-2$ & CBM & GS & $5-12.5$ & Bt1 & 7.7 & 0.52 & 1.2 & 3.2 & 1.1 & 0.8 & 3.6 & 0.5 & 0.7 \\
\hline $3-2$ & CBM & GS & $12.5-24$ & $\mathrm{Bt} 2$ & 7.9 & 0.41 & 1.1 & 2.4 & 0.8 & 0.8 & 2.6 & 0.5 & 0.5 \\
\hline $3-3$ & CBM & $\mathrm{S}$ & $0-6$ & A & 7.5 & 0.76 & 0.8 & 4.6 & 1.6 & 0.4 & 5.9 & 0.5 & 0.3 \\
\hline $3-3$ & CBM & $\mathrm{S}$ & $6-13$ & Bt1 & 7.8 & 0.47 & 1.1 & 3.1 & 1.2 & 0.7 & 3.7 & 0.4 & 0.4 \\
\hline $3-3$ & CBM & $\mathrm{S}$ & 13-23.5 & $\mathrm{Bt} 2$ & 7.9 & 0.5 & 1 & 3.1 & 1.3 & 0.7 & 2.7 & 0.3 & 0.7 \\
\hline $3-4$ & CBM & G & $0-5.5$ & A & 7.4 & 0.83 & 0.8 & 5.9 & 2 & 0.4 & 6.4 & 0.6 & 0.3 \\
\hline $3-4$ & CBM & G & $5.5-13$ & Bt1 & 7.6 & 0.6 & 1.1 & 3.6 & 1.4 & 0.7 & 4.4 & 0.6 & 0.4 \\
\hline $3-4$ & CBM & G & $13-16$ & $\mathrm{Bt} 2$ & 7.8 & 0.6 & 1.2 & 3.6 & 1.3 & 0.8 & 3.6 & 0.6 & 0.7 \\
\hline $4-1$ & CBM & GS & $0-5$ & A & 7.6 & 0.71 & 1 & 4.4 & 1.7 & 0.6 & 5.4 & 0.7 & 0.4 \\
\hline $4-1$ & CBM & GS & $5-14$ & Bt1 & 7.9 & 0.43 & 1 & 2.2 & 0.8 & 0.8 & 2.5 & 0.6 & 0.4 \\
\hline $4-1$ & CBM & GS & $14-24$ & $\mathrm{Bt} 2$ & 7.9 & 0.4 & 0.8 & 2.2 & 0.8 & 0.7 & 2.5 & 0.3 & 0.4 \\
\hline $4-2$ & CBM & NT & $0-4.75$ & A & 7.4 & 0.66 & 1 & 4.6 & 1.6 & 0.6 & 6.2 & 0.2 & 0.5 \\
\hline $4-2$ & CBM & NT & $4.75-12$ & Bt1 & 7.8 & 0.45 & 1 & 2.8 & 0.9 & 0.7 & 3.6 & 0 & 0.3 \\
\hline $4-2$ & CBM & NT & $12-24$ & $\mathrm{Bt} 2$ & 7.9 & 0.41 & 0.9 & 2.5 & 0.8 & 0.7 & 2.4 & 0.2 & 0.3 \\
\hline $4-3$ & CBM & $\mathrm{S}$ & $0-5$ & $\mathrm{~A}$ & 7.3 & 1.11 & 1 & 8.1 & 3 & 0.4 & 10.4 & 0.2 & 0.5 \\
\hline $4-3$ & CBM & $\mathrm{S}$ & $5-13$ & Bt1 & 7.8 & 0.5 & 1.1 & 3.2 & 1.1 & 0.8 & 3.6 & 0.2 & 0.6 \\
\hline $4-3$ & CBM & $\mathrm{S}$ & $13-24$ & $\mathrm{Bt} 2$ & 7.9 & 0.47 & 1.1 & 2.7 & 0.9 & 0.8 & 2.8 & 0.1 & 0.7 \\
\hline $4-4$ & CBM & G & $0-4.5$ & A & 7.3 & 1.07 & 1 & 7.2 & 2.7 & 0.5 & 8.2 & 0.2 & 0.5 \\
\hline $4-4$ & CBM & G & $4.5-13$ & Bt1 & 7.7 & 0.52 & 1.1 & 3.1 & 1 & 0.8 & 3.4 & 0 & 0.6 \\
\hline $4-4$ & CBM & G & $13-21$ & $\mathrm{Bt} 2$ & 7.9 & 0.43 & 1 & 2.4 & 0.8 & 0.8 & 3.2 & 0.5 & 0.6 \\
\hline $5-1$ & CBM + Gyp & GS & $0-5$ & A & 7.6 & 0.63 & 1 & 3.9 & 1.4 & 0.6 & 5.2 & 0.4 & 0.4 \\
\hline $5-1$ & CBM + Gyp & GS & 5-13.5 & Bt1 & 7.7 & 0.59 & 1 & 3.2 & 1.1 & 0.7 & 3.9 & 0.2 & 0.4 \\
\hline $5-1$ & CBM + Gyp & GS & $13.5-24$ & $\mathrm{Bt} 2$ & 7.9 & 0.51 & 1 & 2.6 & 0.9 & 0.8 & 2.8 & 0.2 & 0.5 \\
\hline
\end{tabular}




\begin{tabular}{|c|c|c|c|c|c|c|c|c|c|c|c|c|}
\hline $5-2$ & CBM + Gyp & G & $0-5.5$ & A & 7.4 & 0.73 & 0.8 & 4.6 & 1.6 & 0.5 & 6 & 0.3 \\
\hline $5-2$ & CBM + Gyp & $\mathrm{G}$ & $5.5-13$ & Bt1 & 7.7 & 0.45 & 1.1 & 2.5 & 0.9 & 0.9 & 3.4 & 0.5 \\
\hline $5-2$ & CBM + Gyp & $\mathrm{G}$ & $13-24$ & $\mathrm{Bt} 2$ & 7.8 & 0.48 & 1.1 & 2.5 & 0.9 & 0.9 & 2.6 & 0.3 \\
\hline $5-3$ & CBM + Gyp & NT & $0-6$ & A & 7.4 & 0.76 & 0.8 & 4.7 & 1.6 & 0.5 & 6.6 & 0.2 \\
\hline $5-3$ & CBM + Gyp & NT & $6-13.5$ & Bt1 & 7.7 & 0.41 & 0.9 & 2.3 & 0.8 & 0.7 & 3.4 & 0 \\
\hline $5-3$ & CBM + Gyp & NT & $13.5-24$ & $\mathrm{Bt} 2$ & 7.9 & 0.37 & 1 & 2.1 & 0.7 & 0.8 & 2.4 & 0.2 \\
\hline $5-4$ & CBM + Gyp & $\mathrm{S}$ & $0-6$ & $\mathrm{~A}$ & 7.6 & 0.63 & 0.7 & 3.9 & 1.3 & 0.4 & 5.4 & 0.3 \\
\hline $5-4$ & CBM + Gyp & $\mathrm{S}$ & $6-13$ & Bt1 & 7.8 & 0.41 & 0.7 & 2.6 & 1 & 0.6 & 3.2 & 0.2 \\
\hline $5-4$ & CBM + Gyp & $\mathrm{S}$ & $13-24$ & $\mathrm{Bt} 2$ & 7.9 & 0.41 & 0.9 & 2.3 & 0.9 & 0.7 & 3 & 0.4 \\
\hline $6-1$ & CBM + Gyp & NT & $0-6.5$ & A & 7.5 & 0.7 & 0.9 & 4.6 & 1.5 & 0.5 & 7.8 & 0.5 \\
\hline $6-1$ & CBM + Gyp & NT & 6.5-13.5 & Bt1 & 7.7 & 0.54 & 1.1 & 2.6 & 0.8 & 0.8 & 3 & 0 \\
\hline $6-1$ & CBM + Gyp & NT & $13.5-24$ & $\mathrm{Bt} 2$ & 7.8 & 0.52 & 1 & 2.7 & 0.9 & 0.7 & 3.4 & 0 \\
\hline $6-2$ & CBM + Gyp & $\mathrm{S}$ & $0-5.75$ & A & 7.4 & 0.72 & 0.9 & 5.2 & 1.8 & 0.5 & 5 & 0.1 \\
\hline $6-2$ & CBM + Gyp & S & $5.75-13.5$ & Bt1 & 7.6 & 0.5 & 1.2 & 3.3 & 1.2 & 0.8 & 3.6 & 0.3 \\
\hline $6-2$ & CBM + Gyp & $\mathrm{S}$ & $13.5-24$ & $\mathrm{Bt} 2$ & 7.7 & 0.45 & 1.1 & 2.9 & 1 & 0.8 & 2.4 & 0 \\
\hline $6-3$ & CBM + Gyp & GS & $0-6$ & A & 7.7 & 0.74 & 0.8 & 5.2 & 1.7 & 0.4 & 5.2 & 0.2 \\
\hline $6-3$ & CBM + Gyp & GS & $6-13$ & Bt1 & 7.6 & 0.61 & 1.2 & 3.9 & 1.5 & 0.7 & 5.4 & 0.1 \\
\hline $6-3$ & CBM + Gyp & GS & $13-24$ & $\mathrm{Bt} 2$ & 7.6 & 0.55 & 1.3 & 3.3 & 1.2 & 0.8 & 3.4 & 0.2 \\
\hline $6-4$ & CBM + Gyp & $\mathrm{G}$ & $0-6.25$ & A & 7.5 & 0.6 & 0.7 & 4.2 & 1.5 & 0.4 & 5 & 0.6 \\
\hline $6-4$ & CBM + Gyp & G & $6.25-13.5$ & Bt1 & 7.7 & 0.51 & 1 & 3.3 & 1.2 & 0.6 & 3.9 & 0.3 \\
\hline $6-4$ & CBM + Gyp & G & $13.5-24$ & $\mathrm{Bt} 2$ & 7.9 & 0.4 & 1 & 2.5 & 0.9 & 0.8 & 2.3 & 0.2 \\
\hline $7-1$ & CBM + Gyp + SB & $\mathrm{S}$ & $0-4.5$ & A & 6.9 & 1.04 & 0.6 & 8.6 & 2.6 & 0.3 & 9.5 & 0.4 \\
\hline $7-1$ & $\mathrm{CBM}+\mathrm{Gyp}+\mathrm{SB}$ & $\mathrm{S}$ & $4.5-11$ & Bt1 & 7.3 & 0.64 & 0.9 & 4.7 & 1.6 & 0.5 & 5.7 & 0.1 \\
\hline $7-1$ & $\mathrm{CBM}+\mathrm{Gyp}+\mathrm{SB}$ & $\mathrm{S}$ & $11-14.5$ & Bt2 & 7.5 & 0.54 & 1.2 & 4 & 1.5 & 0.7 & 4.4 & 0.6 \\
\hline $7-2$ & $\mathrm{CBM}+\mathrm{Gyp}+\mathrm{SB}$ & NT & $0-5$ & A & 6.8 & 1.34 & 0.7 & 12.3 & 3.9 & 0.2 & 10.8 & 0.8 \\
\hline $7-2$ & CBM + Gyp + SB & NT & 5-11.25 & Bt1 & 7.4 & 0.63 & 1 & 4.4 & 1.5 & 0.6 & 4.6 & 0.3 \\
\hline $7-2$ & CBM + Gyp + SB & NT & $11.25-24$ & Bt2 & 7.6 & 0.52 & 1.1 & 3.2 & 1.2 & 0.8 & 3 & 0.4 \\
\hline
\end{tabular}




\begin{tabular}{|c|c|c|c|c|c|c|c|c|c|c|c|c|c|}
\hline $7-3$ & CBM + Gyp + SB & GS & $0-5$ & $\mathrm{~A}$ & 7.2 & 0.9 & 0.7 & 6.7 & 2.3 & 0.3 & 8.6 & 0.7 & 0.4 \\
\hline $7-3$ & $\mathrm{CBM}+\mathrm{Gyp}+\mathrm{SB}$ & GS & $5-11.5$ & Bt1 & 7.5 & 0.54 & 1.1 & 4 & 1.4 & 0.6 & 5 & 0.5 & 0.4 \\
\hline $7-3$ & $\mathrm{CBM}+\mathrm{Gyp}+\mathrm{SB}$ & GS & $11.5-24$ & $\mathrm{Bt} 2$ & 7.7 & 0.48 & 1.2 & 2.8 & 1 & 0.8 & 3.6 & 0.3 & 0.7 \\
\hline $7-4$ & $\mathrm{CBM}+\mathrm{Gyp}+\mathrm{SB}$ & $\mathrm{G}$ & $0-4.5$ & $\mathrm{~A}$ & 7.2 & 0.93 & 0.7 & 8 & 2.4 & 0.3 & 8.2 & 0.4 & 0.4 \\
\hline $7-4$ & $\mathrm{CBM}+\mathrm{Gyp}+\mathrm{SB}$ & $\mathrm{G}$ & 4.5-10.5 & Bt1 & 7.6 & 0.42 & 0.9 & 3 & 0.9 & 0.6 & 4.2 & 0.7 & 0.3 \\
\hline $7-4$ & CBM + Gyp + SB & $\mathrm{G}$ & $10.5-24$ & $\mathrm{Bt} 2$ & 7.7 & 0.55 & 1.1 & 3.3 & 1.3 & 0.7 & 3.6 & 0.3 & 0.8 \\
\hline $8-1$ & CBM + Gyp + SB & G & $0-4$ & $\mathrm{~A}$ & 7.3 & 0.78 & 0.7 & 6.4 & 2 & 0.4 & 6.8 & 0.7 & 0.3 \\
\hline $8-1$ & CBM + Gyp + SB & G & $4-10.5$ & Bt1 & 7.5 & 0.41 & 0.7 & 2.3 & 0.8 & 0.6 & 4 & 0.4 & 0.2 \\
\hline 8-1 & CBM + Gyp + SB & $\mathrm{G}$ & $10.5-24$ & $\mathrm{Bt} 2$ & 7.6 & 0.38 & 0.9 & 2.5 & 0.8 & 0.7 & 3.6 & 0.3 & 0.3 \\
\hline $8-2$ & CBM + Gyp + SB & $\mathrm{S}$ & $0-4.25$ & A & 7.3 & 0.73 & 1 & 6 & 2 & 0.5 & 7.4 & 1 & 0.4 \\
\hline $8-2$ & $\mathrm{CBM}+\mathrm{Gyp}+\mathrm{SB}$ & $\mathrm{S}$ & $4.25-11$ & Bt1 & 7.6 & 0.51 & 1.2 & 4 & 1.3 & 0.8 & 5.4 & 0.6 & 0.6 \\
\hline $8-2$ & $\mathrm{CBM}+\mathrm{Gyp}+\mathrm{SB}$ & $\mathrm{S}$ & $11-24$ & $\mathrm{Bt} 2$ & 7.6 & 0.47 & 0.9 & 2.9 & 1.1 & 0.7 & 4.6 & 0.3 & 0.7 \\
\hline $8-3$ & CBM + Gyp + SB & GS & $0-4.5$ & $\mathrm{~A}$ & 7.3 & 0.73 & 0.7 & 5.7 & 1.8 & 0.4 & 7 & 0.4 & 0.5 \\
\hline 8-3 & CBM + Gyp + SB & GS & $4.5-11$ & Bt1 & 7.4 & 0.58 & 1 & 3.9 & 1.2 & 0.6 & 4.4 & 0.4 & 0.3 \\
\hline 8-3 & $\mathrm{CBM}+\mathrm{Gyp}+\mathrm{SB}$ & GS & $11-24$ & $\mathrm{Bt} 2$ & 7.7 & 0.41 & 1 & 2.6 & 0.8 & 0.8 & 3.2 & 0.5 & 0.5 \\
\hline $8-4$ & $\mathrm{CBM}+\mathrm{Gyp}+\mathrm{SB}$ & NT & $0-4.25$ & $\mathrm{~A}$ & 7 & 1.12 & 0.9 & 9.5 & 3 & 0.4 & 9.6 & 0.5 & 0.5 \\
\hline $8-4$ & $\mathrm{CBM}+\mathrm{Gyp}+\mathrm{SB}$ & NT & $4.25-10.5$ & Bt1 & 7.5 & 0.46 & 0.9 & 3.6 & 1.2 & 0.6 & 4.4 & 0.5 & 0.3 \\
\hline $8-4$ & CBM + Gyp + SB & NT & $10.5-24$ & $\mathrm{Bt} 2$ & 7.7 & 0.48 & 0.9 & 3 & 1.1 & 0.6 & 3.6 & 0.4 & 0.8 \\
\hline $9-1$ & $\mathrm{CBM}+\mathrm{Gyp}+\mathrm{SB}$ & $\mathrm{G}$ & $0-4$ & $\mathrm{~A}$ & 7.1 & 1 & 0.7 & 7.9 & 2.5 & 0.3 & 8.4 & 0.5 & 0.5 \\
\hline $9-1$ & CBM + Gyp + SB & G & $4-11$ & Bt1 & 7.5 & 0.4 & 1.1 & 3.3 & 1.1 & 0.7 & 4 & 1.4 & 0.3 \\
\hline $9-1$ & CBM + Gyp + SB & $\mathrm{G}$ & $11-24$ & $\mathrm{Bt} 2$ & 7.7 & 0.5 & 1.3 & 2.7 & 1.1 & 1 & 3 & 0.5 & 0.8 \\
\hline $9-2$ & CBM + Gyp + SB & GS & $0-4.5$ & $\mathrm{~A}$ & 7.1 & 1.16 & 0.7 & 9.4 & 2.6 & 0.3 & 9.2 & 1 & 0.6 \\
\hline $9-2$ & CBM + Gyp + SB & GS & $4.5-9.5$ & Bt1 & 7.4 & 0.75 & 1 & 5.4 & 1.8 & 0.5 & 7.4 & 0.7 & 0.4 \\
\hline $9-2$ & CBM + Gyp + SB & GS & $9.5-24$ & $\mathrm{Bt} 2$ & 7.6 & 0.5 & 1 & 3.3 & 1.1 & 0.6 & 3.8 & 0.8 & 0.5 \\
\hline $9-3$ & CBM + Gyp + SB & $\mathrm{S}$ & $0-4$ & A & 7 & 1.07 & 0.6 & 8.9 & 2.5 & 0.3 & 8.4 & 0.5 & 0.6 \\
\hline $9-3$ & CBM + Gyp + SB & $\mathrm{S}$ & $4-11$ & Bt1 & 7.3 & 0.58 & 0.8 & 4.4 & 1.3 & 0.5 & 4.8 & 0.3 & 0.5 \\
\hline $9-3$ & $\mathrm{CBM}+\mathrm{Gyp}+\mathrm{SB}$ & $\mathrm{S}$ & $11-24$ & $\mathrm{Bt} 2$ & 7.5 & 0.48 & 0.9 & 3.4 & 1.2 & 0.6 & 3.3 & 0.4 & 0.6 \\
\hline
\end{tabular}




\begin{tabular}{|c|c|c|c|c|c|c|c|c|c|c|c|c|c|}
\hline $9-4$ & $\mathrm{CBM}+\mathrm{Gyp}+\mathrm{SB}$ & NT & $0-5$ & A & 7 & 1.03 & 0.6 & 8.8 & 2.6 & 0.3 & 9.2 & 0.7 & 0.6 \\
\hline $9-4$ & $\mathrm{CBM}+\mathrm{Gyp}+\mathrm{SB}$ & NT & $5-12.5$ & Bt1 & 7 & 0.5 & 0.6 & 8.9 & 2.6 & 0.3 & 4.1 & 1 & 0.5 \\
\hline $9-4$ & $\mathrm{CBM}+\mathrm{Gyp}+\mathrm{SB}$ & NT & $12.5-24$ & Bt2 & 7.6 & 0.52 & 0.8 & 4 & 1.3 & 0.5 & 3 & 0.4 & 0.6 \\
\hline $10-1$ & $\mathrm{CBM}+\mathrm{Gyp}+\mathrm{SB}$ & $\mathrm{S}$ & $0-5.5$ & A & 7.1 & 0.89 & 0.6 & 7.9 & 2.3 & 0.2 & 8 & 0.7 & 0.5 \\
\hline $10-1$ & $\mathrm{CBM}+\mathrm{Gyp}+\mathrm{SB}$ & $\mathrm{S}$ & $5.5-14$ & Bt1 & 7.4 & 0.57 & 0.9 & 3.2 & 1.2 & 0.6 & 4.2 & 0.3 & 0.5 \\
\hline $10-1$ & $\mathrm{CBM}+\mathrm{Gyp}+\mathrm{SB}$ & $\mathrm{S}$ & $14-24$ & Bt2 & 7.6 & 0.62 & 0.8 & 4.4 & 1.5 & 0.4 & 3.3 & 0.4 & 1 \\
\hline $10-2$ & $\mathrm{CBM}+\mathrm{Gyp}+\mathrm{SB}$ & G & $0-6$ & A & 7.2 & 0.74 & 1 & 4 & 1.6 & 0.6 & 7.1 & 0.3 & 0.8 \\
\hline $10-2$ & $\mathrm{CBM}+\mathrm{Gyp}+\mathrm{SB}$ & G & $6-14$ & Bt1 & 7.5 & 0.49 & 0.6 & 6.5 & 1.9 & 0.3 & 4.3 & 0.3 & 0.4 \\
\hline $10-2$ & $\mathrm{CBM}+\mathrm{Gyp}+\mathrm{SB}$ & G & $14-24$ & Bt2 & 7.6 & 0.52 & 0.7 & 3.5 & 1.1 & 0.5 & 3.3 & 0.4 & 0.6 \\
\hline $10-3$ & $\mathrm{CBM}+\mathrm{Gyp}+\mathrm{SB}$ & NT & $0-6.25$ & A & 7.3 & 0.68 & 0.9 & 3.3 & 1.3 & 0.6 & 6.4 & 0.1 & 0.3 \\
\hline $10-3$ & $\mathrm{CBM}+\mathrm{Gyp}+\mathrm{SB}$ & NT & $6.25-13.5$ & Bt1 & 7.5 & 0.46 & 0.5 & 5.3 & 1.6 & 0.2 & 3.7 & 0.3 & 0.5 \\
\hline $10-3$ & $\mathrm{CBM}+\mathrm{Gyp}+\mathrm{SB}$ & NT & $13.25-24$ & Bt2 & 7.6 & 0.49 & 0.5 & 3.1 & 1 & 0.4 & 3.4 & 0.2 & 0.5 \\
\hline $10-4$ & $\mathrm{CBM}+\mathrm{Gyp}+\mathrm{SB}$ & GS & $0-6$ & A & 7.2 & 0.78 & 0.6 & 6.7 & 1.9 & 0.3 & 7.1 & 0.5 & 0.4 \\
\hline $10-4$ & $\mathrm{CBM}+\mathrm{Gyp}+\mathrm{SB}$ & GS & $6-14$ & Bt1 & 7.5 & 0.54 & 0.8 & 3.8 & 1.3 & 0.5 & 4.2 & 0.3 & 0.4 \\
\hline $10-4$ & $\mathrm{CBM}+\mathrm{Gyp}+\mathrm{SB}$ & GS & $14-24$ & Bt2 & 7.6 & 0.49 & 1 & 3.3 & 1.2 & 0.6 & 3.6 & 0.5 & 0.8 \\
\hline $11-1$ & PC & NT & $0-6$ & A & 7 & 1.1 & 0.7 & 9.9 & 3.2 & 0.3 & 9 & 0.5 & 0.5 \\
\hline $11-1$ & PC & NT & $6-13$ & Bt1 & 7.4 & 0.76 & 1.1 & 6 & 2.2 & 0.5 & 6 & 0.3 & 0.9 \\
\hline $11-1$ & PC & NT & $13-24$ & Bt2 & 7.4 & 0.74 & 0.7 & 4.8 & 2 & 0.4 & 4.2 & 0.7 & 1.6 \\
\hline $11-2$ & PC & GS & $0-6$ & A & 7.2 & 0.98 & 0.3 & 8.4 & 2.5 & 0.1 & 9.6 & 0.5 & 0.5 \\
\hline $11-2$ & PC & GS & $6-13$ & Bt1 & 7.4 & 0.74 & 0.8 & 5.6 & 1.9 & 0.4 & 6.4 & 0.4 & 0.7 \\
\hline $11-2$ & PC & GS & $13-24$ & Bt2 & 7.6 & 0.61 & 0.6 & 4.1 & 1.4 & 0.4 & 4.2 & 0.4 & 0.9 \\
\hline $11-3$ & PC & $\mathrm{S}$ & $0-5.5$ & A & 7.4 & 0.9 & & 6.4 & 1.9 & & 7.6 & 1 & 0.6 \\
\hline $11-3$ & PC & $\mathrm{S}$ & $5.5-12.5$ & Bt1 & 7.6 & 0.59 & 0.3 & 4.4 & 1.4 & 0.2 & 4.9 & 0.4 & 0.8 \\
\hline $11-3$ & PC & $\mathrm{S}$ & $12.5-24$ & Bt2 & 7.7 & 0.49 & 0.2 & 3.4 & 1.1 & 0.2 & 3.6 & 0.4 & 0.7 \\
\hline $11-4$ & PC & G & $0-6$ & A & 7.4 & 0.84 & & 5.8 & 2.1 & & 8.6 & 0.5 & 0.5 \\
\hline $11-4$ & PC & G & $6-13.5$ & Bt1 & 7.7 & 0.51 & 0.3 & 3.4 & 1.4 & 0.2 & 3.9 & 0.5 & 0.6 \\
\hline $11-4$ & PC & G & $13.5-24$ & Bt2 & 7.7 & 0.55 & 0.4 & 3.4 & 1.4 & 0.3 & 2.9 & 0.5 & 0.9 \\
\hline
\end{tabular}




\begin{tabular}{|c|c|c|c|c|c|c|c|c|c|c|c|c|c|}
\hline $12-1$ & PC & NT & $0-5.5$ & A & 7.3 & 0.93 & 0.3 & 7.3 & 2.7 & 0.2 & 9.3 & 0.4 & 0.5 \\
\hline $12-1$ & PC & NT & 5.5-13.5 & Bt1 & 7.6 & 0.8 & 0.7 & 5.9 & 2.2 & 0.4 & 5.6 & 0.5 & 1.1 \\
\hline $12-1$ & PC & NT & $13.5-24$ & Bt2 & 7.8 & 0.71 & 0.8 & 4.7 & 1.9 & 0.4 & 3.5 & 0.4 & 1.8 \\
\hline $12-2$ & PC & $\mathrm{S}$ & $0-6.5$ & A & 7.1 & 1.09 & 0.2 & 9.5 & 2.9 & & 10.8 & 0.5 & 0.4 \\
\hline $12-2$ & PC & S & $6.5-13$ & Bt1 & 7.6 & 0.77 & 0.6 & 5.2 & 2 & 0.3 & 5.6 & 0.4 & 0.9 \\
\hline $12-2$ & PC & S & $13-24$ & Bt2 & 7.8 & 0.6 & 0.5 & 4 & 1.6 & 0.3 & 3.5 & 1.2 & 1.3 \\
\hline $12-3$ & PC & G & $0-5$ & A & 7.4 & 1 & 0.4 & 6.6 & 2 & 0.2 & 8.7 & 0.3 & 0.6 \\
\hline $12-3$ & PC & G & $5-12.5$ & Bt1 & 7.6 & 0.73 & 0.6 & 4.4 & 1.4 & 0.3 & 4.7 & 0.4 & 0.9 \\
\hline $12-3$ & PC & G & $12.5-24$ & Bt2 & 7.8 & 0.69 & 0.6 & 4.3 & 1.9 & 0.4 & 3.3 & 0.1 & 1.5 \\
\hline $12-4$ & PC & GS & $0-6$ & A & 7.5 & 0.82 & 0.8 & 6.6 & 2 & 0.4 & 7.3 & 0.7 & 0.4 \\
\hline $12-4$ & PC & GS & $6-13.5$ & Bt1 & 7.7 & 0.65 & 0.4 & 4.8 & 1.7 & 0.2 & 5 & 0.4 & 0.7 \\
\hline $12-4$ & PC & GS & $13.5-24$ & Bt2 & 7.8 & 0.52 & 0.4 & 3.5 & 1.3 & 0.2 & 3.7 & 0.4 & 0.8 \\
\hline 13-1 & CBM & GS & $0-5$ & A & 7.1 & 1.2 & 0.2 & 10.7 & 3.4 & & 9.7 & 0.3 & 0.5 \\
\hline $13-1$ & CBM & GS & $5-11.5$ & Bt1 & 7.6 & 0.64 & 0.4 & 4.5 & 1.7 & 0.2 & 5.2 & 0.4 & 0.6 \\
\hline 13-1 & CBM & GS & $11.5-24$ & Bt2 & 7.8 & 0.63 & 0.5 & 4.1 & 1.7 & 0.3 & 3.7 & 0.3 & 1.1 \\
\hline $13-2$ & CBM & NT & $0-5.5$ & A & 7.3 & 1.2 & 1.1 & 8.5 & 3.1 & 0.5 & 9.1 & 0.7 & 0.6 \\
\hline $13-2$ & CBM & NT & 5.5-12 & Bt1 & 7.3 & 0.85 & 1.3 & 5.8 & 2.2 & 0.6 & 5.9 & 0.3 & 1.3 \\
\hline $13-2$ & CBM & NT & $12-24$ & Bt2 & 7.6 & 0.9 & 1.2 & 5.7 & 3 & 0.6 & 8.4 & 0.3 & 2.4 \\
\hline $13-3$ & CBM & $\mathrm{S}$ & $0-5$ & A & 7.2 & 1.4 & & 11 & 4.4 & & 11.5 & 0.5 & 0.5 \\
\hline $13-3$ & CBM & S & $5-11.5$ & Bt1 & 7.4 & 0.82 & 0.7 & 5.8 & 2.6 & 0.4 & 8 & 0.3 & 0.5 \\
\hline $13-3$ & CBM & S & $11.5-24$ & Bt2 & 7.8 & 0.6 & 0.8 & 3.8 & 1.8 & 0.5 & 4.6 & 0.4 & 0.8 \\
\hline $13-4$ & CBM & G & $0-6$ & A & 7.4 & 1.97 & 0.5 & 15.3 & 5.5 & 0.2 & 9.8 & 2.9 & 1.3 \\
\hline $13-4$ & CBM & G & $6-11.5$ & Bt1 & 7.7 & 1.2 & 0.4 & 8.1 & 3.3 & 0.2 & 6.1 & 0.8 & 0.9 \\
\hline $13-4$ & CBM & G & $11.5-24$ & Bt2 & 7.9 & 0.74 & 0.5 & 4.6 & 1.9 & 0.3 & 4.3 & 0.8 & 0.7 \\
\hline $14-1$ & CBM & GS & $0-5.75$ & A & 7.4 & 1.2 & 0.2 & 8.9 & 3.5 & & 10 & 0.8 & 0.5 \\
\hline $14-1$ & CBM & GS & $5.75-12$ & Bt1 & 7.8 & 0.55 & 0.4 & 3.7 & 1.4 & 0.3 & 4.6 & 0.5 & 0.4 \\
\hline $14-1$ & CBM & GS & $12-24$ & Bt2 & 8 & 0.58 & 0.8 & 3.6 & 1.5 & 0.5 & 3 & 0.5 & 1.1 \\
\hline
\end{tabular}




\begin{tabular}{|c|c|c|c|c|c|c|c|c|c|c|c|c|c|}
\hline $14-2$ & CBM & S & $0-5.5$ & A & 7.3 & 1 & 0.3 & 9 & 3.1 & 0.1 & 9 & 0.5 & 0.4 \\
\hline $14-2$ & CBM & S & 5.5-11.5 & Bt1 & 7.7 & 0.65 & 0.5 & 4.8 & 2 & 0.3 & 5.2 & 0.3 & 0.5 \\
\hline $14-2$ & $\mathrm{CBM}$ & $\mathrm{S}$ & $11.5-24$ & Bt2 & 7.9 & 0.6 & 0.6 & 4 & 1.9 & 0.4 & 3.4 & 0.5 & 1.2 \\
\hline $14-3$ & CBM & $\mathrm{G}$ & $0-5$ & $\mathrm{~A}$ & 7.3 & 0.97 & 0.1 & 8.1 & 3.1 & & 8 & 0.3 & 0.4 \\
\hline $14-3$ & CBM & $\mathrm{G}$ & 5-11 & Bt1 & 7.6 & 0.73 & 0.5 & 4.8 & 2.2 & 0.2 & 5.2 & 0.5 & 0.9 \\
\hline $14-3$ & $\mathrm{CBM}$ & G & $11-24$ & Bt2 & 7.9 & 0.58 & 0.8 & 3.5 & 1.7 & 0.5 & 2.8 & 0.3 & 1 \\
\hline $14-4$ & CBM & NT & $0-5.75$ & $\mathrm{~A}$ & 7.5 & 0.9 & 0.2 & 5.8 & 2.3 & & 7.2 & 0.5 & 0.7 \\
\hline $14-4$ & CBM & NT & $5.75-11.5$ & Bt1 & 7.7 & 0.76 & 0.5 & 5.2 & 2.3 & 0.3 & 6.2 & 0.3 & 1.1 \\
\hline $14-4$ & CBM & NT & $11.5-24$ & Bt2 & 7.9 & 0.55 & 0.7 & 3.4 & 1.8 & 0.4 & 4.2 & 0.3 & 0.8 \\
\hline $15-1$ & CBM + Gyp & $\mathrm{G}$ & $0-6$ & A & 7.5 & 0.77 & 0.2 & 5.8 & 2.3 & 0.1 & 7.4 & 0.5 & 0.4 \\
\hline $15-1$ & CBM + Gyp & G & $6-13$ & Bt1 & 7.8 & 0.6 & 0.3 & 4 & 1.8 & 0.2 & 4.6 & 0.5 & 0.9 \\
\hline $15-1$ & CBM + Gyp & G & $13-24$ & Bt2 & 7.8 & 0.58 & 0.5 & 4.1 & 1.9 & 0.3 & 3.6 & 0.5 & 1.1 \\
\hline $15-2$ & CBM + Gyp & NT & $0-6$ & $\mathrm{~A}$ & 7.5 & 0.68 & 0.4 & 4.9 & 2 & 0.2 & 5.2 & 0.5 & 0.5 \\
\hline $15-2$ & CBM + Gyp & NT & $6-12$ & Bt1 & 7.7 & 0.52 & 0.5 & 3.1 & 1.4 & 0.3 & 4.1 & 0.3 & 0.4 \\
\hline $15-2$ & CBM + Gyp & NT & $12-24$ & Bt2 & 7.9 & 0.57 & 0.6 & 3.4 & 1.6 & 0.4 & 3.3 & 0.3 & 0.8 \\
\hline $15-3$ & CBM + Gyp & $\mathrm{S}$ & $0-6$ & A & 7.4 & 0.89 & 0.3 & 7 & 2.5 & 0.1 & 8.2 & 0.7 & 0.4 \\
\hline $15-3$ & CBM + Gyp & S & $6-11$ & Bt1 & 7.7 & 0.65 & 0.6 & 4.5 & 1.8 & 0.3 & 5 & 0.5 & 0.5 \\
\hline $15-3$ & CBM + Gyp & $\mathrm{S}$ & $11-24$ & Bt2 & 7.9 & 0.63 & 0.9 & 3.9 & 1.8 & 0.6 & 3.6 & 0.3 & 1.2 \\
\hline $15-4$ & CBM + Gyp & GS & $0-5$ & A & 7.3 & 0.97 & 0.3 & 6.9 & 2.7 & 0.1 & 7 & 0.8 & 0.4 \\
\hline $15-4$ & CBM + Gyp & GS & $5-11$ & Bt1 & 7.6 & 0.6 & 0.4 & 4.4 & 1.7 & 0.2 & 5.8 & 0.5 & 0.3 \\
\hline $15-4$ & CBM + Gyp & GS & $11-23$ & Bt2 & 7.9 & 0.51 & 0.5 & 3.2 & 1.3 & 0.4 & 3.4 & 0.3 & 0.6 \\
\hline $16-1$ & CBM + Gyp & $\mathrm{G}$ & $0-5$ & A & 7.3 & 1.1 & 0.4 & 9.8 & 3.8 & 0.1 & 10.4 & 0.5 & 0.7 \\
\hline $16-1$ & CBM + Gyp & G & $5-12$ & Bt1 & 7.7 & 0.62 & 0.5 & 4.4 & 1.9 & 0.3 & 4.6 & 0.5 & 0.7 \\
\hline $16-1$ & CBM + Gyp & G & $12-24$ & Bt2 & 7.8 & 0.6 & 1.2 & 4.2 & 1.8 & 0.7 & 3.5 & 0.5 & 1.1 \\
\hline $16-2$ & CBM + Gyp & $\mathrm{S}$ & $0-5$ & $\mathrm{~A}$ & 7.3 & 1.09 & 0.7 & 8.5 & 3.2 & 0.3 & 8.4 & 0.4 & 0.6 \\
\hline $16-2$ & CBM + Gyp & S & $5-12$ & Bt1 & 7.7 & 0.64 & 1 & 4.5 & 1.8 & 0.6 & 4.4 & 0.4 & 0.6 \\
\hline $16-2$ & CBM + Gyp & S & $12-24$ & Bt2 & 7.9 & 0.55 & 1 & 3.8 & 1.5 & 0.6 & 3.9 & 0.4 & 1.1 \\
\hline
\end{tabular}




\begin{tabular}{|c|c|c|c|c|c|c|c|c|c|c|c|c|c|}
\hline $16-3$ & CBM + Gyp & NT & $0-6$ & A & 7.6 & 0.77 & 0.7 & 6.2 & 2 & 0.3 & 6.1 & 0.4 & 0.5 \\
\hline $16-3$ & CBM + Gyp & NT & $6-12$ & Bt1 & 7.6 & 0.64 & 0.9 & 4.9 & 1.9 & 0.5 & 6 & 0.5 & 0.7 \\
\hline $16-3$ & CBM + Gyp & NT & $12-24$ & Bt2 & 7.9 & 0.53 & 1 & 3.7 & 1.5 & 0.6 & 3.1 & 0.5 & 1 \\
\hline $16-4$ & CBM + Gyp & GS & $0-6$ & A & 7.4 & 1.15 & 0.8 & 9.3 & 3.1 & 0.3 & 9.3 & 0.7 & 0.9 \\
\hline $16-4$ & CBM + Gyp & GS & $6-11$ & Bt1 & 7.7 & 0.71 & 0.8 & 5.4 & 1.8 & 0.4 & 5.3 & 0.4 & 0.6 \\
\hline $16-4$ & CBM + Gyp & GS & $11-18$ & Bt2 & 7.9 & 0.63 & 1 & 4.5 & 1.7 & 0.6 & 3.8 & 0.4 & 1.3 \\
\hline $16-4$ & CBM + Gyp & GS & $18-24$ & Bt3 & 7.9 & 0.63 & 1.1 & 4.2 & 1.7 & 0.7 & 3.5 & 0.4 & 1.3 \\
\hline $17-1$ & CBM PC Blend & GS & $0-6$ & $\mathrm{~A}$ & 7.4 & 0.65 & 0.7 & 4.8 & 2.1 & 0.4 & 5.8 & 0.5 & 0.4 \\
\hline $17-1$ & CBM PC Blend & GS & $6-16$ & Bt1 & 7.5 & 0.52 & 1.1 & 3.5 & 1.6 & 0.7 & 4 & 0.4 & 0.7 \\
\hline $17-1$ & CBM PC Blend & GS & $16-24$ & Bt2 & 7.8 & 0.7 & 2.7 & 3.3 & 2.1 & 1.7 & 3.5 & 0.4 & 1.5 \\
\hline $17-2$ & CBM PC Blend & $\mathrm{S}$ & $0-6$ & $\mathrm{~A}$ & 7.3 & 1.1 & 1 & 8.1 & 3.4 & 0.4 & 8.8 & 1.1 & 0.5 \\
\hline $17-2$ & CBM PC Blend & S & $6-15$ & Bt1 & 7.7 & 0.62 & 1.3 & 4.4 & 1.9 & 0.7 & 5.2 & 0.3 & 0.4 \\
\hline $17-2$ & CBM PC Blend & S & $15-24$ & Bt2 & 7.9 & 0.63 & 3.4 & 2.3 & 1.7 & 2.4 & 4.3 & 0.5 & 0.9 \\
\hline $17-3$ & CBM PC Blend & G & $0-6$ & $\mathrm{~A}$ & 7.4 & 0.71 & 0.7 & 5.2 & 2.5 & 0.3 & 6.4 & 0.3 & 0.4 \\
\hline $17-3$ & CBM PC Blend & $\mathrm{G}$ & $6-15$ & Bt1 & 7.6 & 0.65 & 1.4 & 4.4 & 2.2 & 0.8 & 5.5 & 0.8 & 0.6 \\
\hline $17-3$ & CBM PC Blend & G & $15-24$ & Bt2 & 7.9 & 1.04 & 5.7 & 3.3 & 2.6 & 3.3 & 3.8 & 0.5 & 3 \\
\hline $17-4$ & CBM PC Blend & NT & $0-6$ & A & 7.2 & 1.02 & 1 & 7.9 & 3.6 & 0.4 & 9.6 & 0.8 & 0.7 \\
\hline $17-4$ & CBM PC Blend & NT & $6-17$ & Bt1 & 7.6 & 0.81 & 1.9 & 5.4 & 2.5 & 0.9 & 4.6 & 4.9 & 1.6 \\
\hline $17-4$ & CBM PC Blend & NT & $17-24$ & Bt2 & 7.9 & 1.85 & 5.6 & 10.5 & 6.8 & 1.9 & 3.7 & 0.4 & 10 \\
\hline $18-1$ & CBM PC Blend & GS & $0-6$ & $\mathrm{~A}$ & 7.3 & 0.84 & 0.9 & 6.4 & 3 & 0.4 & 7.3 & 0.7 & 0.5 \\
\hline $18-1$ & CBM PC Blend & GS & $6-14$ & Bt1 & 7.6 & 0.65 & 1.2 & 4.2 & 2 & 0.7 & 4.2 & 0.5 & 1 \\
\hline $18-1$ & CBM PC Blend & GS & $14-24$ & Bt2 & 7.5 & 1.53 & 5 & 7.5 & 4.5 & 2 & 3.4 & 0.7 & 5.9 \\
\hline $18-2$ & CBM PC Blend & $\mathrm{S}$ & $0-6$ & $\mathrm{~A}$ & 7.2 & 0.88 & 0.7 & 6.8 & 3.3 & 0.3 & 7.1 & 0.5 & 0.6 \\
\hline $18-2$ & CBM PC Blend & S & $6-15$ & Bt1 & 7.5 & 0.87 & 1.3 & 5.4 & 2.6 & 0.7 & 4.9 & 0.5 & 1.8 \\
\hline $18-2$ & CBM PC Blend & $\mathrm{S}$ & $15-24$ & Bt2 & 7.7 & 1.32 & 6.2 & 4.8 & 3.5 & 3 & 3.6 & 0.5 & 4.8 \\
\hline $18-3$ & CBM PC Blend & G & $0-5$ & $\mathrm{~A}$ & 7.2 & 1.36 & 0.8 & 8.7 & 3.9 & 0.3 & 10.4 & 1 & 0.9 \\
\hline $18-3$ & CBM PC Blend & $\mathrm{G}$ & 5-15 & Bt1 & 7.4 & 1.83 & 1.7 & 12.6 & 6.1 & 0.6 & 5.5 & 1.7 & 2.9 \\
\hline
\end{tabular}


18-3 CBM PC Blend

18-4 CBM PC Blend

18-4 CBM PC Blend

G 15-24

18-4 CBM PC Blend

NT $\quad 0-6$

NT $\quad 6-16$

Bt2 $\quad 7.7 \quad 1.79$

4.4

A $\quad 7.2 \quad 1.04$

0.8

$10.3 \quad 6.8$

Bt1 $7.6 \quad 1.04$

NT 16-24

Bt2 7.7

1.49

1.

8.3

8.3

$\begin{array}{ccccc}3.8 & 0.3 & 8.9 & 0.5 & 0.5 \\ 3.5 & 0.8 & 7 & 0.3 & 2.9 \\ 4.4 & 1.6 & 3.7 & 0.8 & 5.5\end{array}$




\section{Appendix VI}

Soil Chemistry Data from Cooksley Ranch Site - Spring 2004 


\begin{tabular}{|c|c|c|c|c|c|c|c|c|c|c|c|c|c|}
\hline $\begin{array}{c}\text { Plot } \\
\# \\
\end{array}$ & $\begin{array}{l}\text { Irrigation } \\
\text { Treatment }\end{array}$ & $\begin{array}{c}\text { Plot } \\
\text { Treat. }\end{array}$ & Depth, in & $\begin{array}{c}\text { Soil } \\
\text { Horizon }\end{array}$ & $\begin{array}{l}\mathrm{pH} \\
\text { s.u. }\end{array}$ & $\begin{array}{c}\text { EC } \\
\text { mmhos/cm }\end{array}$ & $\begin{array}{c}\mathrm{Na} \\
\mathrm{meq} / \mathrm{L}\end{array}$ & $\begin{array}{c}\mathrm{Ca} \\
\mathrm{meq} / \mathrm{L} \\
\end{array}$ & $\begin{array}{c}\mathrm{Mg} \\
\mathrm{meq} / \mathrm{L}\end{array}$ & SAR & $\begin{array}{c}\text { Alkalinity } \\
\mathrm{mg} / \mathrm{L}\end{array}$ & $\begin{array}{c}\mathrm{Cl} \\
\mathrm{meq} / \mathrm{L}\end{array}$ & $\begin{array}{c}\mathrm{SO} 4 \\
\mathrm{meq} / \mathrm{L} \\
\end{array}$ \\
\hline $1-1$ & PC & GS & $0-4.5$ & $\mathrm{~A}$ & 7.3 & 2.16 & 1.0 & 18.9 & 6.4 & 0.3 & 4.0 & $<0.01$ & 22.7 \\
\hline $1-1$ & PC & GS & $4.5-10.5$ & Bt1 & 7.3 & 1.33 & 1.3 & 9.7 & 4.0 & 0.5 & 4.4 & 0.28 & 10.8 \\
\hline $1-1$ & PC & GS & $10.5-24$ & Bt2 & 7.5 & 1.50 & 3.8 & 7.9 & 3.4 & 1.6 & 3.2 & 0.14 & 12.6 \\
\hline $1-2$ & PC & $\mathrm{G}$ & 0-4 & A & 7.2 & 1.83 & 0.8 & 15.1 & 4.5 & 0.2 & 4.0 & 0.14 & 15.7 \\
\hline $1-2$ & PC & G & $4-11$ & Bt1 & 7.4 & 1.02 & 1.0 & 7.5 & 2.7 & 0.5 & 6.0 & 0.28 & 7.3 \\
\hline $1-2$ & PC & $\mathrm{G}$ & $11-24$ & Bt2 & 7.5 & 0.61 & 1.0 & 4.1 & 1.4 & 0.6 & 2.2 & $<0.01$ & 4.1 \\
\hline $1-3$ & PC & NT & $0-4$ & $\mathrm{~A}$ & 7.3 & 0.67 & 0.8 & 4.6 & 1.6 & 0.4 & 7.4 & 0.56 & 1.1 \\
\hline $1-3$ & PC & NT & $4-11$ & Bt1 & 7.5 & 0.63 & 1.0 & 3.5 & 1.3 & 0.6 & 3.6 & 0.14 & 1.6 \\
\hline $1-3$ & PC & NT & $0-0$ & Bt2 & 7.7 & 0.58 & 1.2 & 3.8 & 1.4 & 0.8 & 3.2 & 0.28 & 3.4 \\
\hline $1-4$ & PC & $\mathrm{S}$ & $0-6$ & $\mathrm{~A}$ & 7.4 & 0.81 & 0.7 & 5.9 & 2.1 & 0.4 & 5.2 & 0.14 & 3.7 \\
\hline $1-4$ & PC & S & $6-11$ & Bt1 & 7.4 & 0.62 & 1.0 & 4.4 & 1.7 & 0.6 & 5.2 & $<0.01$ & 15.9 \\
\hline $1-4$ & PC & $\mathrm{S}$ & $11-24$ & Bt2 & 7.5 & 0.49 & 1.0 & 3.1 & 1.2 & 0.7 & 3.6 & 0.14 & 1.6 \\
\hline $2-1$ & PC & $\mathrm{G}$ & $0-4.5$ & $\mathrm{~A}$ & 7.3 & 1.93 & 1.0 & 14.8 & 5.2 & 0.3 & 3.2 & 0.56 & 18.3 \\
\hline $2-1$ & PC & G & 4.5-11 & Bt1 & 7.3 & 0.97 & 1.1 & 6.8 & 2.8 & 0.5 & 3.6 & $<0.01$ & 7.1 \\
\hline $2-1$ & PC & G & $11-24$ & Bt2 & 7.6 & 0.49 & 1.1 & 3.0 & 1.3 & 0.7 & 3.2 & 0.28 & 1.8 \\
\hline $2-2$ & PC & NT & $0-5$ & $\mathrm{~A}$ & 7.4 & 0.57 & 0.8 & 4.3 & 1.5 & 0.5 & 4.4 & 0.28 & 1.4 \\
\hline $2-2$ & PC & NT & $5-10$ & Bt1 & 7.4 & 0.54 & 1.0 & 3.8 & 1.4 & 0.6 & 4.6 & 0.14 & 1.6 \\
\hline $2-2$ & PC & NT & $10-21$ & Bt2 & 7.5 & 0.61 & 1.1 & 3.9 & 1.5 & 0.7 & 2.8 & 1.13 & 1.5 \\
\hline $2-3$ & PC & $\mathrm{S}$ & $0-6.5$ & $\mathrm{~A}$ & 7.5 & 0.73 & 0.9 & 5.2 & 1.8 & 0.4 & 3.4 & 0.14 & 5.5 \\
\hline $2-3$ & PC & $S$ & 6.5-12 & Bt1 & 7.5 & 0.57 & 1.0 & 3.7 & 1.4 & 0.6 & 3.6 & 0.14 & 2.4 \\
\hline $2-3$ & PC & $\mathrm{S}$ & $12-24$ & Bt2 & 7.7 & 0.55 & 1.3 & 3.2 & 1.2 & 0.8 & 2.8 & 0.28 & 2.7 \\
\hline $2-4$ & PC & GS & $0-4.5$ & $\mathrm{~A}$ & 7.4 & 2.38 & 0.8 & 20.0 & 5.9 & 0.2 & 3.6 & 0.28 & 26.1 \\
\hline $2-4$ & PC & GS & 4.5-11 & Bt1 & 7.5 & 0.98 & 1.1 & 6.9 & 2.5 & 0.5 & 4.0 & 0.28 & 6.4 \\
\hline $2-4$ & CBM & NT & $11-24$ & Bt2 & 7.7 & 0.63 & 1.2 & 3.8 & 1.5 & 0.7 & 2.8 & $<0.01$ & 3.6 \\
\hline
\end{tabular}




\begin{tabular}{|c|c|c|c|c|c|c|c|c|c|c|c|c|c|}
\hline $3-1$ & CBM & NT & $0-6$ & A & 7.5 & 0.70 & 0.9 & 4.7 & 1.5 & 0.5 & 4.4 & 0.28 & 3.1 \\
\hline $3-1$ & CBM & NT & $6-13$ & Bt1 & 7.6 & 0.60 & 0.9 & 3.1 & 1.0 & 0.7 & 3.2 & $<0.01$ & 1.7 \\
\hline $3-1$ & CBM & GS & $0-0$ & Bt2 & 7.7 & 0.47 & 0.9 & 3.0 & 1.0 & 0.6 & 2.8 & 0.14 & 1.8 \\
\hline $3-2$ & CBM & GS & $0-6$ & $\mathrm{~A}$ & 7.4 & 1.83 & 0.9 & 14.7 & 4.2 & 0.3 & 3.2 & 0.14 & 17.6 \\
\hline $3-2$ & CBM & GS & $6-11.5$ & Bt1 & 7.5 & 1.33 & 1.0 & 10.4 & 3.3 & 0.4 & 4.4 & $<0.01$ & 10.4 \\
\hline $3-2$ & CBM & $\mathrm{S}$ & $11.5-24$ & Bt2 & 7.5 & 0.97 & 1.1 & 7.0 & 2.3 & 0.5 & 3.8 & 0.14 & 6.5 \\
\hline $3-3$ & CBM & S & $0-6.5$ & $\mathrm{~A}$ & 7.5 & 0.73 & 0.8 & 5.6 & 1.9 & 0.4 & 4.8 & 0.28 & 3.5 \\
\hline $3-3$ & CBM & S & 6.5-12 & Bt1 & 7.6 & 0.48 & 0.8 & 3.3 & 1.2 & 0.6 & 4.4 & $<0.01$ & 1.6 \\
\hline $3-3$ & CBM & G & $12-24$ & Bt2 & 7.7 & 0.46 & 1.0 & 3.1 & 1.3 & 0.7 & 3.8 & 0.14 & 1.8 \\
\hline $3-4$ & CBM & G & $0-5$ & $\mathrm{~A}$ & 7.3 & 1.57 & 0.7 & 10.9 & 3.2 & 0.3 & 4.6 & 0.14 & 12.0 \\
\hline $3-4$ & CBM & G & $5-10$ & Bt1 & 7.4 & 1.14 & 0.9 & 8.2 & 2.8 & 0.4 & 4.2 & 0.28 & 8.1 \\
\hline $3-4$ & CBM & GS & $10-27$ & Bt2 & 7.5 & 1.06 & 1.3 & 8.1 & 3.0 & 0.5 & 3.8 & 0.42 & 8.2 \\
\hline 4-1 & CBM & GS & $0-5$ & $\mathrm{~A}$ & 7.4 & 1.38 & 0.6 & 10 & 2.7 & 0.2 & 4.8 & 0.42 & 10.5 \\
\hline 4-1 & CBM & GS & $5-14$ & Bt1 & 7.5 & 0.62 & 0.5 & 4.3 & 1.4 & 0.3 & 3.8 & 0.14 & 3.1 \\
\hline 4-1 & CBM & NT & $14-23$ & Bt2 & 7.6 & 0.52 & 0.7 & 3.4 & 1.1 & 0.5 & 2.6 & 0.28 & 2.6 \\
\hline 4-2 & CBM & NT & $0-4.5$ & $\mathrm{~A}$ & 7.3 & 0.84 & 0.8 & 5.9 & 2.1 & 0.4 & 6.2 & 1.13 & 1.4 \\
\hline $4-2$ & CBM & NT & 4.5-11 & Bt1 & 7.5 & 0.58 & 0.7 & 4.1 & 1.3 & 0.4 & 5.8 & 0.56 & 1.3 \\
\hline $4-2$ & CBM & $\mathrm{S}$ & $11-24$ & Bt2 & 7.6 & 0.45 & 0.8 & 3.1 & 1.0 & 0.5 & 3.0 & 0.71 & 1.5 \\
\hline 4-3 & CBM & $S$ & $0-5$ & A & 7.5 & 0.82 & 0.8 & 5.8 & 1.8 & 0.4 & 5.2 & 0.42 & 1.2 \\
\hline $4-3$ & CBM & S & 5-11 & Bt1 & 7.5 & 0.52 & 0.8 & 3.7 & 1.3 & 0.5 & 4.0 & 0.28 & 5.2 \\
\hline 4-3 & CBM & G & $11-24$ & Bt2 & 7.7 & 0.57 & 0.9 & 3.5 & 1.5 & 0.6 & 3.8 & $<0.01$ & 1.4 \\
\hline 4-4 & $\mathrm{CBM}$ & $\mathrm{G}$ & $0-4.5$ & $\mathrm{~A}$ & 7.5 & 0.98 & 0.7 & 7.1 & 2.2 & 0.3 & 4.6 & 0.14 & 2.7 \\
\hline 4-4 & CBM & $\mathrm{G}$ & $4.5-13$ & Bt1 & 7.7 & 0.46 & 0.9 & 3.0 & 1.1 & 0.6 & 2.4 & 0.14 & 5.6 \\
\hline 4-4 & CBM & $\mathrm{G}$ & $13-24$ & Bt2 & 7.6 & 0.63 & 1.0 & 5.1 & 1.8 & 0.5 & 3.8 & 0.28 & 2.3 \\
\hline $5-1$ & CBM + Gyp & GS & $0-5.5$ & $\mathrm{~A}$ & 7.0 & 2.78 & 1.2 & 30.2 & 7.5 & 0.3 & 2.2 & 0.68 & 33.2 \\
\hline $5-1$ & CBM + Gyp & GS & $5.5-13$ & Bt1 & 7.1 & 1.36 & 1.0 & 10.1 & 3.2 & 0.4 & 3.0 & 0.25 & 9.7 \\
\hline $5-1$ & CBM + Gyp & GS & $13-24$ & $\mathrm{Bt} 2$ & 7.4 & 0.73 & 1.1 & 5.6 & 1.8 & 0.6 & 2.4 & 0.25 & 5.0 \\
\hline
\end{tabular}




\begin{tabular}{|c|c|c|c|c|c|c|c|c|c|c|c|c|c|}
\hline $5-2$ & CBM + Gyp & G & $0-5$ & A & 7.1 & 2.74 & 1.0 & 25.1 & 7.3 & 0.3 & 2.6 & 0.54 & 28.4 \\
\hline $5-2$ & CBM + Gyp & G & $5-13$ & Bt1 & 7.2 & 1.40 & 1.2 & 10.1 & 3.7 & 0.5 & 4.6 & 0.25 & 9.7 \\
\hline $5-2$ & CBM + Gyp & G & $13-24$ & Bt2 & 7.3 & 0.74 & 1.2 & 5.4 & 2.1 & 0.6 & 4.4 & 0.39 & 3.2 \\
\hline $5-3$ & CBM + Gyp & NT & $0-5.5$ & $\mathrm{~A}$ & 7.2 & 0.77 & 0.8 & 5.1 & 1.7 & 0.4 & 4.4 & 0.54 & 1.1 \\
\hline $5-3$ & CBM + Gyp & NT & $5.5-11.5$ & Bt1 & 7.3 & 0.57 & 0.8 & 3.7 & 1.4 & 0.5 & 2.6 & 0.39 & 0.8 \\
\hline $5-3$ & CBM + Gyp & NT & $11.5-24$ & Bt2 & 7.5 & 0.44 & 0.7 & 3.0 & 1.2 & 0.5 & 3.0 & 0.39 & 1.0 \\
\hline $5-4$ & CBM + Gyp & $\mathrm{S}$ & 0-6 & $\mathrm{A}$ & 7.0 & 1.48 & 1.0 & 12.7 & 3.8 & 0.3 & 4.6 & 0.39 & 12.5 \\
\hline $5-4$ & CBM + Gyp & S & $6-12$ & Bt1 & 7.3 & 1.37 & 0.9 & 11.8 & 3.5 & 0.3 & 3.4 & 0.54 & 11.6 \\
\hline $5-4$ & CBM + Gyp & $\mathrm{S}$ & $12-24$ & Bt2 & 7.4 & 0.70 & 0.9 & 5.5 & 2.0 & 0.4 & 6.0 & 0.54 & 3.1 \\
\hline $6-1$ & CBM + Gyp & NT & $0-6.5$ & $\mathrm{~A}$ & 7.4 & 0.54 & 1.0 & 4.4 & 1.6 & 0.6 & 4.8 & 0.54 & 2.2 \\
\hline $6-1$ & CBM + Gyp & NT & 6.5-13.5 & Bt1 & 7.3 & 0.60 & 1.0 & 4.9 & 1.6 & 0.6 & 4.8 & 0.25 & 1.6 \\
\hline $6-1$ & CBM + Gyp & NT & $13.5-24$ & Bt2 & 7.6 & 0.48 & 0.9 & 3.5 & 1.2 & 0.6 & 2.8 & 0.39 & 1.5 \\
\hline $6-2$ & CBM + Gyp & $\mathrm{S}$ & $0-5$ & A & 7.2 & 1.03 & 1.0 & 8.6 & 2.7 & 0.4 & 3.4 & 0.39 & 6.1 \\
\hline $6-2$ & CBM + Gyp & S & $5-12$ & Bt1 & 7.4 & 0.58 & 0.7 & 4.6 & 1.6 & 0.4 & 5.0 & 0.39 & 1.8 \\
\hline $6-2$ & CBM + Gyp & $\mathrm{S}$ & $12-24$ & Bt2 & 7.5 & 0.52 & 0.8 & 3.6 & 1.3 & 0.5 & 2.6 & 0.39 & 1.7 \\
\hline $6-3$ & CBM + Gyp & GS & $0-? ?$ & $\mathrm{~A}$ & 7.2 & 1.95 & 1.0 & 19.4 & 5.6 & 0.3 & 1.0 & 0.39 & 19.8 \\
\hline $6-3$ & CBM + Gyp & GS & ??-13.5 & Bt1 & 7.3 & 1.34 & 0.9 & 10.5 & 4.0 & 0.3 & 5.6 & 0.25 & 10.5 \\
\hline $6-3$ & CBM + Gyp & GS & $13.5-24$ & Bt2 & 7.6 & 0.82 & 1.1 & 6.4 & 2.7 & 0.5 & 2.4 & 0.39 & 6.7 \\
\hline $6-4$ & CBM + Gyp & $\mathrm{G}$ & 0-6 & A & 7.3 & 1.26 & 0.8 & 10.5 & 3.0 & 0.3 & 3.0 & 0.54 & 10.0 \\
\hline $6-4$ & CBM + Gyp & G & $6-12$ & Bt1 & 7.4 & 1.26 & 0.9 & 11.0 & 3.9 & 0.3 & 5.2 & 0.39 & 9.1 \\
\hline $6-4$ & CBM + Gyp & G & $12-24$ & Bt2 & 7.6 & 0.98 & 0.8 & 7.9 & 3.0 & 0.4 & 2.6 & 0.25 & 8.1 \\
\hline $7-1$ & CBM + Gyp + SB & S & $0-5$ & $\mathrm{~A}$ & 7.3 & 1.13 & 1.0 & 8.8 & 2.6 & 0.4 & 4.8 & 0.39 & 6.9 \\
\hline $7-1$ & $\mathrm{CBM}+\mathrm{Gyp}+\mathrm{SB}$ & S & $5-11$ & Bt1 & 7.4 & 0.66 & 1.1 & 5.1 & 1.7 & 0.6 & 3.2 & 0.68 & 2.7 \\
\hline $7-1$ & $\mathrm{CBM}+\mathrm{Gyp}+\mathrm{SB}$ & $\mathrm{S}$ & $11-24$ & Bt2 & 7.6 & 0.57 & 1.1 & 4.0 & 1.4 & 0.7 & 4.2 & 0.39 & 2.5 \\
\hline $7-2$ & CBM + Gyp + SB & NT & $0-5$ & A & 7.4 & 0.56 & 0.8 & 4.5 & 1.4 & 0.5 & 6.6 & 0.68 & 1.0 \\
\hline $7-2$ & $\mathrm{CBM}+\mathrm{Gyp}+\mathrm{SB}$ & NT & $5-11$ & Bt1 & 7.4 & 0.52 & 1.0 & 4.2 & 1.4 & 0.6 & 5.6 & 0.39 & 1.0 \\
\hline $7-2$ & $\mathrm{CBM}+\mathrm{Gyp}+\mathrm{SB}$ & NT & $11-18$ & $\mathrm{Bt} 2$ & 7.6 & 0.47 & 1.1 & 3.3 & 1.1 & 0.8 & 3.4 & 0.39 & 1.7 \\
\hline
\end{tabular}




\begin{tabular}{|c|c|c|c|c|c|c|c|c|c|c|c|c|c|}
\hline $7-3$ & CBM + Gyp + SB & GS & $0-5$ & A & 7.2 & 2.08 & 0.9 & 21.6 & 5.6 & 0.2 & 3.2 & 0.39 & 22.5 \\
\hline $7-3$ & CBM + Gyp + SB & GS & $5-12$ & Bt1 & 7.5 & 1.30 & 1.5 & 11.0 & 3.6 & 0.6 & 4.8 & 0.39 & 11.8 \\
\hline $7-3$ & CBM + Gyp + SB & GS & $12-23$ & Bt2 & 7.7 & 0.59 & 1.3 & 4.1 & 1.6 & 0.8 & 2.8 & 0.54 & 3.5 \\
\hline $7-4$ & $\mathrm{CBM}+\mathrm{Gyp}+\mathrm{SB}$ & $\mathrm{G}$ & $0-4$ & A & 7.3 & 1.94 & 0.9 & 18.8 & 4.8 & 0.3 & 5.0 & 0.39 & 18.7 \\
\hline $7-4$ & $\mathrm{CBM}+\mathrm{Gyp}+\mathrm{SB}$ & G & $4-11$ & Bt1 & 7.4 & 1.26 & 1.3 & 10.7 & 3.3 & 0.5 & 4.2 & 0.39 & 10.8 \\
\hline $7-4$ & CBM + Gyp + SB & G & $11-24$ & Bt2 & 7.6 & 0.66 & 1.3 & 4.9 & 1.7 & 0.7 & 3.4 & 0.25 & 4.0 \\
\hline 8-1 & CBM + Gyp + SB & G & $0-4$ & $\mathrm{~A}$ & 7.3 & 1.74 & 0.8 & 16.6 & 4.4 & 0.2 & 3.6 & 0.54 & 15.5 \\
\hline $8-1$ & $\mathrm{CBM}+\mathrm{Gyp}+\mathrm{SB}$ & G & $4-11$ & Bt1 & 7.4 & 1.29 & 1.5 & 10.4 & 3.2 & 0.6 & 3.8 & 0.25 & 9.4 \\
\hline $8-1$ & $\mathrm{CBM}+\mathrm{Gyp}+\mathrm{SB}$ & G & $11-24$ & Bt2 & 7.5 & 1.36 & 1.2 & 10.4 & 3.0 & 0.5 & 2.8 & 0.68 & 9.4 \\
\hline $8-2$ & CBM + Gyp + SB & S & $0-4$ & $\mathrm{~A}$ & 7.2 & 2.42 & 1.1 & 19.9 & 5.9 & 0.3 & 3.8 & 1.24 & 11.0 \\
\hline $8-2$ & CBM + Gyp + SB & S & $4-12$ & Bt1 & 7.4 & 1.34 & 1.4 & 10.2 & 3.3 & 0.5 & 3.6 & 1.24 & 6.4 \\
\hline $8-2$ & CBM + Gyp + SB & $\mathrm{S}$ & $12-24$ & Bt2 & 7.5 & 0.55 & 1.0 & 4.3 & 1.5 & 0.6 & 3.6 & 0.68 & 2.1 \\
\hline $8-3$ & $\mathrm{CBM}+\mathrm{Gyp}+\mathrm{SB}$ & GS & $0-5$ & A & 7.3 & 2.38 & 0.6 & 27.7 & 5.9 & 0.2 & 3.2 & 0.39 & 27.9 \\
\hline $8-3$ & CBM + Gyp + SB & GS & $5-11$ & Bt1 & 7.4 & 1.09 & 0.8 & 9.1 & 2.7 & 0.3 & 2.8 & 0.25 & 7.8 \\
\hline $8-3$ & CBM + Gyp + SB & GS & $11-24$ & Bt2 & 7.7 & 0.65 & 0.9 & 5.1 & 1.7 & 0.5 & 2.4 & 0.39 & 4.0 \\
\hline $8-4$ & $\mathrm{CBM}+\mathrm{Gyp}+\mathrm{SB}$ & NT & $0-4$ & $\mathrm{~A}$ & 7.3 & 0.58 & 0.7 & 4.7 & 1.4 & 0.4 & 5.4 & 0.54 & 0.9 \\
\hline $8-4$ & CBM + Gyp + SB & NT & 4-11.5 & Bt1 & 7.4 & 0.57 & 1.0 & 4.4 & 1.5 & 0.6 & 3.6 & 0.54 & 1.5 \\
\hline $8-4$ & CBM + Gyp + SB & NT & $11.5-24$ & Bt2 & 7.6 & 0.51 & 0.9 & 3.8 & 1.4 & 0.6 & 3.5 & 0.25 & 1.9 \\
\hline 9-1 & $\mathrm{CBM}+\mathrm{Gyp}+\mathrm{SB}$ & $\mathrm{G}$ & 0-6 & A & 7.4 & 1.97 & 1.0 & 18.9 & 4.7 & 0.3 & 2.9 & 0.54 & 18.7 \\
\hline $9-1$ & $\mathrm{CBM}+\mathrm{Gyp}+\mathrm{SB}$ & G & $6-12$ & Bt1 & 7.4 & 0.83 & 0.9 & 7.1 & 2.2 & 0.4 & 2.6 & 0.39 & 5.3 \\
\hline $9-1$ & CBM + Gyp + SB & $\mathrm{G}$ & $12-24$ & Bt2 & 7.6 & 0.77 & 1.3 & 6.2 & 2.2 & 0.6 & 2.2 & 0.25 & 6.2 \\
\hline $9-2$ & CBM + Gyp + SB & GS & $0-4$ & A & 7.2 & 1.79 & 0.7 & 17.0 & 4.1 & 0.2 & 3.4 & 0.54 & 15.7 \\
\hline $9-2$ & $\mathrm{CBM}+\mathrm{Gyp}+\mathrm{SB}$ & GS & $4-9.5$ & Bt1 & 7.3 & 1.11 & 0.8 & 10 & 3.0 & 0.3 & 2.8 & 1.38 & 7.6 \\
\hline $9-2$ & $\mathrm{CBM}+\mathrm{Gyp}+\mathrm{SB}$ & GS & $9.5-24$ & Bt2 & 7.6 & 0.74 & 0.9 & 6.0 & 2.0 & 0.5 & 3.2 & 0.54 & 5.9 \\
\hline $9-3$ & CBM + Gyp + SB & $\mathrm{S}$ & $0-4$ & $\mathrm{~A}$ & 7.3 & 0.97 & 1.0 & 7.5 & 2.2 & 0.4 & 6.0 & 0.68 & 5.1 \\
\hline $9-3$ & $\mathrm{CBM}+\mathrm{Gyp}+\mathrm{SB}$ & S & $4-11.5$ & Bt1 & 7.1 & 0.63 & 1.0 & 4.0 & 1.2 & 0.6 & 4.2 & 0.34 & 1.4 \\
\hline $9-3$ & $\mathrm{CBM}+\mathrm{Gyp}+\mathrm{SB}$ & $\mathrm{S}$ & $11.5-22$ & $\mathrm{Bt} 2$ & 7.4 & 0.61 & 1.2 & 3.4 & 1.2 & 0.8 & 2.6 & 0.20 & 2.1 \\
\hline
\end{tabular}




\begin{tabular}{|c|c|c|c|c|c|c|c|c|c|c|c|c|c|}
\hline $9-4$ & $\mathrm{CBM}+\mathrm{Gyp}+\mathrm{SB}$ & NT & $0-6$ & A & 7.2 & 0.75 & 0.9 & 5.7 & 1.6 & 0.4 & 6.2 & 0.48 & 1.7 \\
\hline $9-4$ & $\mathrm{CBM}+\mathrm{Gyp}+\mathrm{SB}$ & NT & $6-13$ & Bt1 & 7.4 & 0.69 & 1.1 & 4.5 & 1.5 & 0.6 & 4.0 & 0.20 & 1.8 \\
\hline $9-4$ & $\mathrm{CBM}+\mathrm{Gyp}+\mathrm{SB}$ & NT & $13-24$ & Bt2 & 7.5 & 0.45 & 1.1 & 3.1 & 1.2 & 0.8 & 3.6 & 0.13 & 2.0 \\
\hline $10-1$ & $\mathrm{CBM}+\mathrm{Gyp}+\mathrm{SB}$ & $\mathrm{S}$ & $0-5.5$ & A & 7.3 & 0.82 & 0.9 & 7.1 & 2.0 & 0.4 & 5.4 & 0.62 & 5.1 \\
\hline $10-1$ & $\mathrm{CBM}+\mathrm{Gyp}+\mathrm{SB}$ & $\mathrm{S}$ & $5.5-8.5$ & Bt1 & 7.2 & 0.57 & 0.9 & 4.7 & 1.4 & 0.5 & 4.4 & 0.34 & 1.3 \\
\hline $10-1$ & $\mathrm{CBM}+\mathrm{Gyp}+\mathrm{SB}$ & $\mathrm{S}$ & $8.5-24$ & Bt2 & 7.5 & 0.50 & 1.0 & 3.7 & 1.3 & 0.7 & 3.4 & 0.41 & 2.0 \\
\hline $10-2$ & $\mathrm{CBM}+\mathrm{Gyp}+\mathrm{SB}$ & G & $0-6$ & A & 7.2 & 0.91 & 0.6 & 7.8 & 2.0 & 0.3 & 5.4 & 0.27 & 4.8 \\
\hline $10-2$ & $\mathrm{CBM}+\mathrm{Gyp}+\mathrm{SB}$ & G & $6-9$ & Bt1 & 7.4 & 0.61 & 0.7 & 5.0 & 1.5 & 0.4 & 4.2 & 0.34 & 3.4 \\
\hline $10-2$ & $\mathrm{CBM}+\mathrm{Gyp}+\mathrm{SB}$ & G & $9-24$ & Bt2 & 7.5 & 0.65 & 0.8 & 4.8 & 1.6 & 0.5 & 2.4 & 0.27 & 4.5 \\
\hline $10-3$ & $\mathrm{CBM}+\mathrm{Gyp}+\mathrm{SB}$ & NT & $0-6$ & A & 7.4 & 0.64 & 0.9 & 5.3 & 1.6 & 0.5 & 6.2 & 0.39 & 1.4 \\
\hline $10-3$ & $\mathrm{CBM}+\mathrm{Gyp}+\mathrm{SB}$ & NT & $6-11$ & Bt1 & 7.4 & 0.66 & 1.0 & 4.7 & 1.5 & 0.6 & 4.8 & 0.41 & 1.4 \\
\hline $10-3$ & $\mathrm{CBM}+\mathrm{Gyp}+\mathrm{SB}$ & NT & $11-24$ & Bt2 & 7.5 & 0.49 & 0.9 & 3.7 & 1.3 & 0.6 & 4.0 & 0.20 & 1.6 \\
\hline $10-4$ & $\mathrm{CBM}+\mathrm{Gyp}+\mathrm{SB}$ & GS & $0-6$ & A & 7.3 & 2.11 & 0.9 & 20.6 & 5.2 & 0.2 & 5.6 & 1.54 & 22.0 \\
\hline $10-4$ & $\mathrm{CBM}+\mathrm{Gyp}+\mathrm{SB}$ & GS & $6-12$ & Bt1 & 7.2 & 1.26 & 1.0 & 11.2 & 3.4 & 0.4 & 5.2 & 0.27 & 9.0 \\
\hline $10-4$ & $\mathrm{CBM}+\mathrm{Gyp}+\mathrm{SB}$ & GS & $12-21.5$ & Bt2 & 7.5 & 1.02 & 1.4 & 7.9 & 2.6 & 0.6 & 2.8 & 0.34 & 7.7 \\
\hline $11-1$ & PC & NT & $0-6.5$ & A & 7.3 & 0.67 & 0.9 & 5.5 & 1.7 & 0.4 & 7.0 & 0.34 & 0.9 \\
\hline $11-1$ & PC & NT & $6.5-11.5$ & Bt1 & 7.3 & 0.64 & 1.2 & 5.0 & 1.6 & 0.6 & 5.6 & 0.27 & 1.6 \\
\hline $11-1$ & PC & NT & $11.5-17$ & Bt2 & 7.5 & 0.65 & 1.2 & 5.0 & 1.7 & 0.7 & 4.6 & 0.20 & 3.4 \\
\hline $11-2$ & PC & GS & $0-4$ & A & 7.3 & 2.14 & 0.8 & 22.7 & 5.7 & 0.2 & 5.0 & 1.89 & 25.5 \\
\hline $11-2$ & PC & GS & $4-12$ & Bt1 & 7.3 & 1.43 & 1.3 & 12.5 & 4.0 & 0.5 & 4.6 & 1.11 & 11.3 \\
\hline $11-2$ & PC & GS & $12-24$ & Bt2 & 7.6 & 0.97 & 1.4 & 7.0 & 2.6 & 0.7 & 5.6 & 0.41 & 7.0 \\
\hline $11-3$ & PC & $\mathrm{S}$ & $0-7$ & A & 7.3 & 1.04 & 1.1 & 8.7 & 2.7 & 0.5 & 7.8 & 0.62 & 5.7 \\
\hline $11-3$ & PC & $\mathrm{s}$ & $7-15$ & Bt1 & 7.5 & 0.80 & 1.3 & 5.9 & 2.1 & 0.6 & 4.6 & 1.75 & 3.3 \\
\hline $11-3$ & PC & $\mathrm{s}$ & $15-24$ & Bt2 & 7.6 & 0.73 & 1.3 & 4.6 & 1.8 & 0.7 & 3.2 & 1.25 & 3.0 \\
\hline $11-4$ & PC & G & $0-7$ & A & 7.2 & 1.95 & 0.8 & 17.8 & 4.7 & 0.2 & 5.2 & 0.41 & 17.3 \\
\hline $11-4$ & PC & G & $7-15$ & Bt1 & 7.4 & 1.38 & 1.4 & 11.1 & 4.0 & 0.5 & 4.2 & 0.34 & 11.3 \\
\hline $11-4$ & PC & G & $15-24$ & Bt2 & 7.6 & 0.79 & 1.3 & 5.2 & 2.0 & 0.7 & 3.0 & 0.34 & 4.8 \\
\hline
\end{tabular}




\begin{tabular}{|c|c|c|c|c|c|c|c|c|c|c|c|c|c|}
\hline $12-1$ & PC & NT & $0-7$ & A & 7.3 & 0.70 & 0.8 & 5.2 & 1.7 & 0.4 & 5.6 & 0.48 & 1.4 \\
\hline $12-1$ & PC & NT & 7-11 & Bt1 & 7.4 & 0.71 & 1.0 & 5.4 & 1.9 & 0.5 & 5.4 & 0.34 & 2.1 \\
\hline $12-1$ & PC & NT & $11-24$ & Bt2 & 7.6 & 0.53 & 1.1 & 3.7 & 1.4 & 0.7 & 3.4 & 0.20 & 2.5 \\
\hline $12-2$ & PC & $\mathrm{S}$ & $0-7$ & A & 7.5 & 1.00 & 0.9 & 8.1 & 2.4 & 0.4 & 6.2 & 1.18 & 6.5 \\
\hline $12-2$ & PC & $\mathrm{S}$ & $7-13$ & Bt1 & 7.5 & 0.62 & 0.9 & 4.5 & 1.5 & 0.5 & 4.6 & 0.48 & 2.0 \\
\hline $12-2$ & PC & $\mathrm{S}$ & $13-24$ & Bt2 & 7.6 & 0.60 & 1.0 & 4.3 & 1.6 & 0.6 & 3.2 & 0.34 & 3.0 \\
\hline $12-3$ & PC & G & $0-5.5$ & A & 7.2 & 3.08 & 1.0 & 29.3 & 6.7 & 0.2 & 5.6 & 0.69 & 30.4 \\
\hline $12-3$ & PC & G & $5.5-12.5$ & Bt1 & 7.4 & 1.62 & 1.2 & 11.5 & 3.5 & 0.4 & 4.2 & 0.48 & 12.0 \\
\hline $12-3$ & PC & G & $12.5-24$ & Bt2 & 7.5 & 1.11 & 1.1 & 8.9 & 3.4 & 0.4 & 3.8 & 0.20 & 9.2 \\
\hline $12-4$ & PC & GS & $0-6$ & A & 7.3 & 2.26 & 0.8 & 23.7 & 6.0 & 0.2 & 5.0 & 1.33 & 25.4 \\
\hline $12-4$ & PC & GS & $6-9$ & Bt1 & 7.4 & 1.40 & 1.0 & 10.8 & 3.5 & 0.4 & 4.2 & 0.48 & 10.7 \\
\hline $12-4$ & PC & GS & $9-24$ & Bt2 & 7.6 & 0.75 & 1.1 & 5.6 & 1.9 & 0.6 & 3.5 & 0.34 & 5.4 \\
\hline $13-1$ & CBM & GS & $0-5$ & A & 7.3 & 2.62 & 0.9 & 24.8 & 7.0 & 0.2 & 9.1 & 2.81 & 24.7 \\
\hline $13-1$ & CBM & GS & $15-13$ & Bt1 & 7.4 & 1.44 & 1.0 & 12.6 & 4.0 & 0.4 & 4.4 & 0.76 & 13.3 \\
\hline $13-1$ & CBM & GS & $13-24$ & Bt2 & 7.6 & 1.03 & 1.3 & 7.8 & 2.8 & 0.6 & 2.8 & 0.48 & 8.8 \\
\hline $13-2$ & CBM & NT & $0-5$ & A & 7.3 & 0.80 & 0.8 & 6.1 & 2.3 & 0.4 & 7.6 & 0.62 & 1.2 \\
\hline $13-2$ & CBM & NT & $5-10.5$ & Bt1 & 7.4 & 0.72 & 1.0 & 6.0 & 2.2 & 0.5 & 7.0 & 0.41 & 1.8 \\
\hline $13-2$ & CBM & NT & $10.5-24$ & Bt2 & 7.6 & 0.61 & 1.1 & 4.3 & 1.8 & 0.6 & 3.6 & 0.20 & 2.7 \\
\hline $13-3$ & CBM & $\mathrm{S}$ & $0-5.5$ & A & 7.4 & 1.05 & 0.8 & 8.8 & 2.9 & 0.3 & 5.9 & 0.90 & 8.5 \\
\hline $13-3$ & CBM & S & $5.5-12$ & Bt1 & 7.4 & 0.71 & 0.9 & 5.8 & 2.1 & 0.5 & 5.1 & 0.34 & 2.7 \\
\hline $13-3$ & CBM & $\mathrm{S}$ & $12-24$ & Bt2 & 7.6 & 0.68 & 1.2 & 5.0 & 2.1 & 0.6 & 4.4 & 0.20 & 3.8 \\
\hline $13-4$ & CBM & G & $0-6$ & A & 7.2 & 2.60 & 0.8 & 27.7 & 7.9 & 0.2 & 6.6 & 0.55 & 28.4 \\
\hline $13-4$ & CBM & G & $6-9$ & Bt1 & 7.2 & 1.77 & 1.2 & 14.5 & 5.4 & 0.4 & 5.2 & 0.41 & 13.7 \\
\hline $13-4$ & CBM & G & $9-24$ & Bt2 & 7.6 & 0.91 & 1.3 & 6.8 & 2.8 & 0.6 & 4.2 & 0.41 & 6.4 \\
\hline $14-1$ & CBM & GS & $0-6$ & A & 7.4 & 1.45 & 0.9 & 11.6 & 3.8 & 0.3 & 6.2 & 1.54 & 11.7 \\
\hline $14-1$ & CBM & GS & $6-11.5$ & Bt1 & 7.4 & 1.10 & 1.0 & 8.9 & 3.0 & 0.4 & 5.2 & 1.18 & 8.1 \\
\hline $14-1$ & CBM & GS & $11.5-21$ & Bt2 & 7.7 & 0.83 & 1.4 & 6.0 & 2.5 & 0.7 & 3.4 & 0.48 & 6.4 \\
\hline
\end{tabular}




\begin{tabular}{|c|c|c|c|c|c|c|c|c|c|c|c|c|c|}
\hline $14-2$ & CBM & $\mathrm{S}$ & $0-6$ & A & 7.5 & 1.03 & 1.0 & 8.7 & 3.1 & 0.4 & 6.0 & 1.33 & 8.8 \\
\hline $14-2$ & CBM & $\mathrm{S}$ & $6-12.5$ & Bt1 & 7.4 & 0.58 & 1.1 & 4.5 & 1.7 & 0.6 & 6.0 & 0.27 & 1.7 \\
\hline $14-2$ & CBM & $\mathrm{S}$ & $12.5-19$ & Bt2 & 7.6 & 0.60 & 1.2 & 4.2 & 1.8 & 0.7 & 4.2 & 0.41 & 2.5 \\
\hline $14-3$ & CBM & G & $0-4.5$ & A & 7.2 & 0.98 & 0.8 & 8.2 & 3.0 & 0.3 & 6.8 & 0.55 & 5.4 \\
\hline $14-3$ & CBM & G & $4.5-9$ & Bt1 & 7.1 & 1.01 & 1.2 & 8.6 & 3.5 & 0.5 & 3.8 & 0.41 & 8.0 \\
\hline $14-3$ & CBM & G & $9-17$ & Bt2 & 7.4 & 1.25 & 1.7 & 8.8 & 4.1 & 0.7 & 3.6 & 1.11 & 10.1 \\
\hline $14-4$ & CBM & NT & $0-5$ & A & 7.4 & 0.82 & 1.2 & 6.6 & 2.3 & 0.6 & 6.6 & 0.41 & 2.2 \\
\hline $14-4$ & CBM & NT & $5-12$ & Bt1 & 7.5 & 0.66 & 1.3 & 5.9 & 2.1 & 0.7 & 4.8 & 0.41 & 3.4 \\
\hline $14-4$ & CBM & NT & $12-24$ & Bt2 & 7.7 & 0.52 & 1.3 & 3.5 & 1.5 & 0.8 & 4.4 & 0.41 & 2.0 \\
\hline $15-1$ & $\mathrm{CBM}+\mathrm{Gyp}$ & G & $0-5.5$ & A & 7.3 & 1.86 & 1.1 & 17.5 & 5.5 & 0.3 & 4.0 & 0.48 & 19.0 \\
\hline $15-1$ & $\mathrm{CBM}+\mathrm{Gyp}$ & G & $5.5-15$ & Bt1 & 7.6 & 1.04 & 1.4 & 8.2 & 3.2 & 0.6 & 4.0 & 0.27 & 8.8 \\
\hline $15-1$ & $\mathrm{CBM}+\mathrm{Gyp}$ & G & $15-24$ & Bt2 & 7.8 & 0.62 & 1.2 & 4.2 & 1.8 & 0.7 & 3.2 & 0.34 & 3.7 \\
\hline $15-2$ & $\mathrm{CBM}+\mathrm{Gyp}$ & NT & $0-6$ & A & 7.4 & 0.63 & 1.0 & 4.9 & 1.9 & 0.6 & 6.8 & 0.41 & 1.1 \\
\hline $15-2$ & $\mathrm{CBM}+\mathrm{Gyp}$ & NT & 6-11.5 & Bt1 & 7.6 & 0.48 & 1.2 & 3.5 & 1.5 & 0.7 & 4.2 & 0.27 & 1.6 \\
\hline $15-2$ & $\mathrm{CBM}+\mathrm{Gyp}$ & NT & $11.5-24$ & Bt2 & 7.8 & 0.51 & 1.4 & 3.6 & 1.6 & 0.8 & 3.8 & 0.34 & 3.0 \\
\hline $15-3$ & $\mathrm{CBM}+\mathrm{Gyp}$ & $\mathrm{S}$ & $0-6$ & A & 7.5 & 0.91 & 1.0 & 7.9 & 2.7 & 0.4 & 6.8 & 0.83 & 7.2 \\
\hline $15-3$ & $\mathrm{CBM}+\mathrm{Gyp}$ & $\mathrm{S}$ & 6-15.5 & Bt1 & 7.7 & 0.65 & 1.5 & 4.9 & 1.9 & 0.8 & 4.4 & 0.55 & 3.3 \\
\hline $15-3$ & $\mathrm{CBM}+\mathrm{Gyp}$ & $\mathrm{S}$ & $15.5-24$ & Bt2 & 7.9 & 0.58 & 1.5 & 3.8 & 1.8 & 0.9 & 3.3 & 0.27 & 3.5 \\
\hline $15-4$ & $\mathrm{CBM}+\mathrm{Gyp}$ & GS & $0-6$ & A & 7.4 & 2.10 & 1.4 & 21.4 & 6.4 & 0.4 & 6.0 & 1.68 & 25.4 \\
\hline $15-4$ & $\mathrm{CBM}+\mathrm{Gyp}$ & GS & 6-16.5 & Bt1 & 7.6 & 1.11 & 1.6 & 9.3 & 3.5 & 0.6 & 4.0 & 0.27 & 9.7 \\
\hline $15-4$ & $\mathrm{CBM}+\mathrm{Gyp}$ & GS & $16.5-24$ & Bt2 & 7.8 & 0.79 & 2.0 & 5.5 & 2.6 & 1.0 & 2.4 & 0.41 & 6.9 \\
\hline $16-1$ & CBM + Gyp & G & $0-6.5$ & A & 7.3 & 2.14 & 1.4 & 21.2 & 6.3 & 0.4 & 5.2 & 0.55 & 21.5 \\
\hline $16-1$ & CBM + Gyp & G & 6.510 .5 & Bt1 & 7.5 & 1.26 & 1.8 & 9.7 & 3.7 & 0.7 & 5.4 & 0.27 & 9.3 \\
\hline $16-1$ & CBM + Gyp & G & $10.5-20$ & Bt2 & 7.8 & 0.78 & 1.7 & 6.0 & 2.5 & 0.8 & 3.4 & 0.41 & 6.2 \\
\hline $16-2$ & $\mathrm{CBM}+\mathrm{Gyp}$ & $\mathrm{S}$ & $0-5.5$ & A & 7.5 & 0.93 & 1.3 & 8.4 & 3.1 & 0.5 & 6.4 & 0.97 & 6.4 \\
\hline $16-2$ & $\mathrm{CBM}+\mathrm{Gyp}$ & $\mathrm{S}$ & 5.512 .5 & Bt1 & 7.6 & 0.60 & 1.5 & 4.8 & 2.0 & 0.8 & 4.4 & 0.41 & 2.7 \\
\hline $16-2$ & $\mathrm{CBM}+\mathrm{Gyp}$ & $\mathrm{S}$ & $12.5-23$ & Bt2 & 7.9 & 0.67 & 1.9 & 4.5 & 2.0 & 1.1 & 3.4 & 0.41 & 4.1 \\
\hline
\end{tabular}




\begin{tabular}{|c|c|c|c|c|c|c|c|c|c|c|c|c|c|}
\hline $16-3$ & CBM + Gyp & NT & $0-5$ & A & 7.4 & 0.77 & 1.1 & 6.3 & 2.4 & 0.5 & 9.0 & 0.62 & 1.0 \\
\hline $16-3$ & CBM + Gyp & NT & $5-13.5$ & Bt1 & 7.6 & 0.60 & 1.5 & 4.5 & 1.7 & 0.8 & 5.4 & 0.48 & 1.2 \\
\hline $16-3$ & CBM + Gyp & NT & $13.5-24$ & Bt2 & 7.9 & 0.56 & 1.7 & 3.6 & 1.5 & 1.1 & 4.4 & 0.34 & 2.6 \\
\hline $16-4$ & CBM + Gyp & GS & $0-4.5$ & A & 7.4 & 2.13 & 1.3 & 18.3 & 5.2 & 0.4 & 7.6 & 1.18 & 19.9 \\
\hline $16-4$ & CBM + Gyp & GS & 4.515 & Bt1 & 7.6 & 0.99 & 1.4 & 8.1 & 2.7 & 0.6 & 6.0 & 0.48 & 7.0 \\
\hline $16-4$ & CBM + Gyp & GS & $15-24$ & Bt2 & 7.8 & 1.01 & 1.8 & 7.9 & 2.9 & 0.8 & 4.6 & 0.41 & 9.0 \\
\hline $17-1$ & CBM PC Blend & GS & $0-4$ & A & 7.2 & 1.97 & 2.7 & 14.7 & 5.9 & 0.8 & 6.0 & 1.25 & 20.0 \\
\hline $17-1$ & CBM PC Blend & GS & $4-16$ & Bt1 & 7.6 & 0.96 & 1.8 & 5.2 & 2.3 & 1.0 & 3.2 & 0.55 & 4.7 \\
\hline $17-1$ & CBM PC Blend & GS & $16-24$ & Bt2 & 7.8 & 1.01 & 3.3 & 5.7 & 2.9 & 1.6 & 3.2 & 0.69 & 7.2 \\
\hline $17-2$ & CBM PC Blend & $\mathrm{S}$ & $0-5$ & A & 7.4 & 1.02 & 2.4 & 7.0 & 2.8 & 1.1 & 5.6 & 0.55 & 5.4 \\
\hline $17-2$ & CBM PC Blend & $\mathrm{s}$ & $5-15$ & Bt1 & 7.6 & 0.61 & 2.5 & 4.0 & 1.8 & 1.5 & 4.6 & 0.27 & 2.6 \\
\hline $17-2$ & CBM PC Blend & $\mathrm{S}$ & $15-24$ & Bt2 & 8.0 & 1.82 & 11.7 & 4.9 & 4.3 & 5.4 & 4.8 & 0.62 & 14.2 \\
\hline $17-3$ & CBM PC Blend & G & $0-5$ & A & 7.4 & 0.66 & 2.2 & 4.6 & 2.0 & 1.2 & 5.2 & 0.69 & 2.6 \\
\hline $17-3$ & CBM PC Blend & G & $5-16$ & Bt1 & 7.6 & 0.80 & 3.0 & 5.0 & 2.5 & 1.6 & 4.0 & 0.48 & 5.3 \\
\hline $17-3$ & CBM PC Blend & G & $16-24$ & Bt2 & 8.0 & 1.77 & 9.1 & 6.1 & 4.7 & 3.9 & 3.6 & 0.41 & 15.3 \\
\hline $17-4$ & CBM PC Blend & NT & $0-4$ & A & 7.4 & 0.75 & 2.6 & 4.7 & 2.3 & 1.4 & 6.0 & 0.55 & 2.1 \\
\hline $17-4$ & CBM PC Blend & NT & $4-14$ & Bt1 & 7.5 & 0.60 & 1.9 & 3.9 & 1.9 & 1.1 & 5.2 & 0.48 & 2.1 \\
\hline $17-4$ & CBM PC Blend & NT & $14-24$ & Bt2 & 8.0 & 1.52 & 7.0 & 5.8 & 3.7 & 3.2 & 3.8 & 0.34 & 12.1 \\
\hline $18-1$ & CBM PC Blend & GS & $0-6$ & A & 7.5 & 1.27 & 2.4 & 9.9 & 3.8 & 0.9 & 4.2 & 0.54 & 10.3 \\
\hline $18-1$ & CBM PC Blend & GS & $6-13$ & Bt1 & 7.6 & 1.08 & 2.5 & 8.0 & 3.6 & 1.0 & 4.4 & 0.48 & 8.4 \\
\hline $18-1$ & CBM PC Blend & GS & $13-19$ & Bt2 & 7.8 & 1.12 & 4.0 & 7.1 & 3.4 & 1.7 & 2.8 & 0.34 & 10.0 \\
\hline $18-2$ & CBM PC Blend & $\mathrm{S}$ & $0-5$ & A & 7.4 & 1.24 & 2.9 & 8.2 & 3.6 & 1.2 & 5.0 & 1.04 & 9.8 \\
\hline $18-2$ & CBM PC Blend & $\mathrm{s}$ & $5-10$ & Bt1 & 7.6 & 0.74 & 1.9 & 4.8 & 2.2 & 1.0 & 6.0 & 0.55 & 3.0 \\
\hline $18-2$ & CBM PC Blend & $\mathrm{s}$ & $10-17$ & Bt2 & 7.6 & 0.66 & 2.3 & 4.3 & 2.1 & 1.3 & 3.6 & 0.48 & 3.7 \\
\hline $18-3$ & CBM PC Blend & G & $0-6.5$ & A & 7.3 & 1.09 & 2.4 & 7.7 & 3.2 & 1.0 & 5.4 & 0.62 & 6.9 \\
\hline $18-3$ & CBM PC Blend & G & $6.5-12$ & Bt1 & 7.4 & 1.08 & 2.6 & 8.1 & 4.0 & 1.0 & 3.6 & 0.34 & 9.6 \\
\hline $18-3$ & CBM PC Blend & G & $12-20$ & Bt2 & 7.7 & 1.31 & 5.0 & 6.7 & 4.0 & 2.2 & 3.4 & 0.54 & 11.1 \\
\hline
\end{tabular}




\begin{tabular}{|c|c|c|c|c|c|c|c|c|c|c|c|c|}
\hline $18-4$ & CBM PC Blend & NT & $0-6$ & A & 7.4 & 0.76 & 2.4 & 5.3 & 2.2 & 1.3 & 6.6 & 0.69 \\
\hline $18-4$ & CBM PC Blend & NT & 6-11.5 & Bt1 & 7.5 & 0.74 & 2.3 & 5.5 & 2.3 & 1.2 & 4.0 & 0.62 \\
\hline $18-4$ & CBM PC Blend & NT & 11.5-19.5 & Bt2 & 7.9 & 0.73 & 2.8 & 4.3 & 1.9 & 1.6 & 5.8 & 0.55 \\
\hline
\end{tabular}




\section{Appendix VII}

Soil Chemistry Data from Cooksley Ranch Site - Fall 2004. 


\begin{tabular}{|c|c|c|c|c|c|c|c|c|c|c|c|c|c|}
\hline $\begin{array}{c}\text { Plot } \\
\# \\
\end{array}$ & $\begin{array}{l}\text { Irrigation } \\
\text { Treatment }\end{array}$ & $\begin{array}{c}\text { Plot } \\
\text { Treatment }\end{array}$ & Depth & $\begin{array}{c}\text { Soil } \\
\text { Horizon }\end{array}$ & $\begin{array}{l}\mathrm{pH} \\
\text { s.u. }\end{array}$ & $\begin{array}{c}\text { EC } \\
\text { mmhos/cm }\end{array}$ & $\begin{array}{c}\mathrm{Na} \\
\mathrm{meq} / \mathrm{L}\end{array}$ & $\begin{array}{c}\mathrm{Ca} \\
\mathrm{meq} / \mathrm{L} \\
\end{array}$ & $\begin{array}{c}\mathrm{Mg} \\
\mathrm{meq} / \mathrm{L}\end{array}$ & SAR & $\begin{array}{c}\text { Alkalinity } \\
\mathrm{mg} / \mathrm{L}\end{array}$ & $\begin{array}{c}\mathrm{Cl} \\
\mathrm{meq} / \mathrm{L}\end{array}$ & $\begin{array}{c}\mathrm{SO} 4 \\
\mathrm{meq} / \mathrm{L}\end{array}$ \\
\hline $1-1$ & PC & GS & $0-4.5$ & $\mathrm{~A}$ & 6.9 & 1.57 & 1.46 & 15.3 & 4.47 & 0.46 & 6.0 & 0.90 & 12.6 \\
\hline $1-1$ & PC & GS & 4.5-12 & Bt1 & 7.0 & 0.88 & 1.26 & 6.51 & 2.67 & 0.59 & 3.7 & 0.44 & 5.78 \\
\hline $1-1$ & PC & GS & $12-24$ & $\mathrm{Bt} 2$ & 8.45 & 1.694 & 1.65 & 13.62 & 5.54 & 0.53 & 2.6 & 0.25 & 17.16 \\
\hline $1-2$ & PC & $\mathrm{G}$ & $0-4.75$ & $\mathrm{~A}$ & 6.9 & 1.06 & 1.18 & 9.09 & 2.80 & 0.48 & 5.7 & 0.53 & 6.71 \\
\hline $1-2$ & PC & $\mathrm{G}$ & $4.75-12$ & $\mathrm{Bt} 1$ & 8.61 & 0.94 & 0.88 & 6.70 & 3.12 & 0.40 & 3.8 & 0.22 & 5.41 \\
\hline $1-2$ & PC & G & $12-24$ & $\mathrm{Bt} 2$ & 8.48 & 1.467 & 1.72 & 10.83 & 4.79 & 0.62 & 2.4 & 0.26 & 14.08 \\
\hline $1-3$ & $\mathrm{PC}$ & NT & $0-4.75$ & A & 8.69 & 1.017 & 1.20 & 7.74 & 2.91 & 0.52 & 6.6 & 0.40 & 1.69 \\
\hline $1-3$ & PC & NT & $4.75-12.5$ & Bt1 & 8.65 & 0.955 & 1.24 & 7.25 & 2.82 & 0.55 & 5 & 0.20 & 4.20 \\
\hline $1-3$ & PC & NT & $12.5-24$ & $\mathrm{Bt} 2$ & 8.59 & 0.73 & 1.33 & 5.15 & 2.17 & 0.70 & 4.4 & 0.18 & 2.65 \\
\hline $1-4$ & PC & $\mathrm{S}$ & 0-6 & A & 6.9 & 1.41 & 1.40 & 11.2 & 4.07 & 0.51 & 5.6 & 0.54 & 10.5 \\
\hline $1-4$ & PC & S & $6-13$ & Bt1 & 8.55 & 1.131 & 1.13 & 8.82 & 3.27 & 0.46 & 5.2 & 3.00 & 5.89 \\
\hline $1-4$ & $\mathrm{PC}$ & $\mathrm{S}$ & $13-24$ & $\mathrm{Bt} 2$ & 8.52 & 8.49 & 1.13 & 5.97 & 2.28 & 0.56 & 4.2 & 0.19 & 3.26 \\
\hline $2-1$ & PC & $\mathrm{G}$ & $0-4.5$ & $\mathrm{~A}$ & 6.8 & 1.52 & 2.19 & 14.2 & 4.32 & 0.72 & 5.9 & 0.75 & 12.2 \\
\hline $2-1$ & PC & $\mathrm{G}$ & $4.5-11$ & Bt1 & 8.66 & 1.058 & 1.67 & 7.31 & 3.28 & 0.73 & 4.2 & 0.30 & 6.75 \\
\hline $2-1$ & PC & G & $11-24$ & $\mathrm{Bt} 2$ & 8.3 & 2.15 & 2.51 & 17.87 & 7.92 & 0.70 & 6 & 0.38 & 21.10 \\
\hline $2-2$ & PC & NT & $0-5$ & A & 6.9 & 0.86 & 2.50 & 5.92 & 2.20 & 1.2 & 6.9 & 0.67 & 2.08 \\
\hline $2-2$ & PC & NT & $5-11$ & Bt1 & 7.0 & 0.75 & 2.23 & 5.32 & 1.98 & 1.2 & 4.7 & 0.57 & 2.84 \\
\hline $2-2$ & PC & NT & $11-24$ & $\mathrm{Bt} 2$ & 7.0 & 0.75 & 1.95 & 5.24 & 1.89 & 1.0 & 4.0 & 0.52 & 3.87 \\
\hline $2-3$ & PC & $\mathrm{S}$ & $0-5.5$ & $\mathrm{~A}$ & 6.9 & 1.59 & 2.17 & 12.9 & 4.50 & 0.73 & 4.9 & 0.56 & 14.2 \\
\hline $2-3$ & PC & S & 5.5-11 & $\mathrm{Bt} 1$ & 7.2 & 0.79 & 1.89 & 5.89 & 2.03 & 0.95 & 3.0 & 0.46 & 6.34 \\
\hline $2-3$ & $\mathrm{PC}$ & $\mathrm{S}$ & $11-24$ & $\mathrm{Bt} 2$ & 7.1 & 1.10 & 1.83 & 8.38 & 3.17 & 0.76 & 2.9 & 0.31 & 9.47 \\
\hline $2-4$ & $\mathrm{PC}$ & GS & $0-6$ & A & 8.43 & 2.4 & 1.84 & 22.80 & 6.53 & 0.48 & 10.4 & 0.57 & 23.47 \\
\hline $2-4$ & PC & GS & 6-10.5 & Bt1 & 7.0 & 2.17 & 2.51 & 21.1 & 7.69 & 0.66 & 3.3 & 0.47 & 25.8 \\
\hline $2-4$ & PC & GS & $10.5-24$ & Bt2 & 7.0 & 2.49 & 2.71 & 23.7 & 9.30 & 0.67 & 2.5 & 0.40 & 31.4 \\
\hline
\end{tabular}




\begin{tabular}{|c|c|c|c|c|c|c|c|c|c|c|c|c|c|}
\hline 3-1 & CBM & NT & $0-5$ & A & 7.1 & 1.47 & 11.5 & 4.37 & 1.48 & 6.8 & 11.6 & 1.75 & 3.63 \\
\hline $3-1$ & CBM & NT & 5-12.5 & Bt1 & 7.0 & 0.79 & 4.51 & 3.60 & 1.17 & 2.9 & 5.5 & 0.80 & 2.29 \\
\hline 3-1 & CBM & NT & $12.5-24$ & Bt2 & 7.1 & 0.70 & 2.36 & 4.27 & 1.33 & 1.4 & 3.3 & 0.66 & 3.24 \\
\hline 3-2 & CBM & GS & $0-5$ & $\mathrm{~A}$ & 7.1 & 2.63 & 15.0 & 14.9 & 3.84 & 4.9 & 6.5 & 0.93 & 24.1 \\
\hline 3-2 & CBM & GS & 5-10.5 & Bt1 & 7.2 & 1.96 & 7.99 & 12.8 & 4.33 & 2.7 & 4.2 & 0.81 & 19.1 \\
\hline $3-2$ & CBM & GS & $10.5-24$ & Bt2 & 7.1 & 2.65 & 4.84 & 26.1 & 10.8 & 1.1 & 2.1 & 1.12 & 36.1 \\
\hline $3-3$ & CBM & $\mathrm{S}$ & 0-6 & A & 7.1 & 1.91 & 13.5 & 8.88 & 2.67 & 5.6 & 6.9 & 0.85 & 16.9 \\
\hline $3-3$ & CBM & S & $6-10$ & Bt1 & 7.2 & 1.40 & 6.14 & 7.16 & 2.44 & 2.8 & 5.6 & 0.69 & 7.86 \\
\hline $3-3$ & CBM & $\mathrm{S}$ & $10-24$ & $\mathrm{Bt} 2$ & 7.1 & 1.44 & 3.01 & 10.7 & 4.59 & 1.1 & 2.7 & 0.67 & 13.3 \\
\hline $3-4$ & CBM & G & $0-5.5$ & A & 7.2 & 1.94 & 12.0 & 8.43 & 2.32 & 5.2 & 7.2 & 0.78 & 13.5 \\
\hline $3-4$ & CBM & $\mathrm{G}$ & $5.5-12.5$ & Bt1 & 7.2 & 2.17 & 6.18 & 14.9 & 5.28 & 1.9 & 3.5 & 0.60 & 21.4 \\
\hline $3-4$ & CBM & $\mathrm{G}$ & $12.5-24$ & Bt2 & 7.0 & 2.64 & 4.88 & 26.2 & 9.97 & 1.2 & 4.0 & 0.88 & 34.1 \\
\hline 4-1 & CBM & GS & $0-5$ & $\mathrm{~A}$ & 7.0 & 3.01 & 12.0 & 25.2 & 7.57 & 3.0 & 6.3 & 0.79 & 34.9 \\
\hline 4-1 & CBM & GS & 5-11.5 & Bt1 & 6.9 & 2.45 & 4.83 & 24.5 & 8.41 & 1.2 & 3.0 & 0.83 & 32.2 \\
\hline $4-1$ & CBM & GS & $11.5-24$ & $\mathrm{Bt} 2$ & 7.0 & 1.72 & 2.03 & 17.0 & 5.72 & 0.60 & 2.5 & 0.52 & 20.2 \\
\hline 4-2 & CBM & NT & $0-4.75$ & A & 7.2 & 1.42 & 12.1 & 3.68 & 1.14 & 7.8 & 10.6 & 1.14 & 1.94 \\
\hline 4-2 & CBM & NT & $4.75-10$ & Bt1 & 7.2 & 0.76 & 4.14 & 3.70 & 1.20 & 2.6 & 4.3 & 0.62 & 2.08 \\
\hline $4-2$ & CBM & NT & $10-24$ & Bt2 & 7.1 & 0.74 & 1.78 & 4.96 & 1.63 & 0.98 & 3.6 & 0.51 & 3.98 \\
\hline $4-3$ & CBM & $\mathrm{S}$ & $0-5$ & A & 7.2 & 2.37 & 18.4 & 8.70 & 2.97 & 7.6 & 11.1 & 1.26 & 14.4 \\
\hline $4-3$ & CBM & $\mathrm{S}$ & $5-12$ & Bt1 & 7.2 & 0.94 & 4.27 & 5.07 & 1.77 & 2.3 & 5.2 & 0.53 & 4.73 \\
\hline $4-3$ & CBM & $\mathrm{S}$ & $12-24$ & Bt2 & 7.2 & 1.07 & 2.17 & 9.10 & 3.83 & 0.85 & 3.3 & 0.39 & 10.3 \\
\hline 4-4 & CBM & G & $0-4.5$ & A & 7.2 & 1.68 & 10.1 & 8.81 & 2.52 & 4.2 & 6.3 & 0.71 & 13.3 \\
\hline 4-4 & CBM & G & $4.5-11$ & Bt1 & 7.0 & 2.67 & 4.58 & 26.6 & 9.71 & 1.1 & 3.8 & 0.78 & 34.6 \\
\hline 4-4 & CBM & $\mathrm{G}$ & $11-24$ & $\mathrm{Bt} 2$ & 7.1 & 1.59 & 2.16 & 13.9 & 5.28 & 0.70 & 2.4 & 0.44 & 17.0 \\
\hline $5-1$ & CBM + Gyp & GS & $0-5$ & A & 6.9 & 2.50 & 14.7 & 13.8 & 3.25 & 5.0 & 9.0 & 1.03 & 16.5 \\
\hline $5-1$ & CBM + Gyp & GS & $5-12$ & Bt1 & 7.2 & 1.67 & 9.37 & 9.40 & 3.10 & 3.8 & 4.7 & 0.71 & 15.0 \\
\hline $5-1$ & CBM + Gyp & GS & $12-24$ & Bt2 & 7.1 & 1.98 & 2.99 & 17.2 & 5.74 & 0.88 & 2.1 & 0.59 & 22.5 \\
\hline
\end{tabular}




\begin{tabular}{|c|c|c|c|c|c|c|c|c|c|c|c|c|c|}
\hline $5-2$ & CBM + Gyp & G & $0-5.5$ & A & 6.9 & 3.36 & 13.4 & 24.9 & 4.70 & 3.5 & 7.9 & 0.84 & 31.9 \\
\hline $5-2$ & CBM + Gyp & G & $5.5-12$ & Bt1 & 7.2 & 2.79 & 11.0 & 16.4 & 5.30 & 3.3 & 3.6 & 0.64 & 27.2 \\
\hline $5-2$ & CBM + Gyp & G & $12-24$ & Bt2 & 7.7 & 2.36 & 3.89 & 18.6 & 7.06 & 1.1 & 2.0 & 0.61 & 25.2 \\
\hline $5-3$ & CBM + Gyp & NT & $0-6$ & $\mathrm{~A}$ & 7.0 & 1.74 & 10.5 & 7.42 & 2.31 & 4.8 & 10.4 & 0.73 & 6.19 \\
\hline $5-3$ & CBM + Gyp & NT & $6-12$ & Bt1 & 7.2 & 1.32 & 3.54 & 8.67 & 3.21 & 1.4 & 4.8 & 0.62 & 9.52 \\
\hline $5-3$ & CBM + Gyp & NT & $12-24$ & Bt2 & 7.2 & 0.95 & 1.64 & 7.42 & 3.13 & 0.71 & 2.2 & 0.56 & 7.96 \\
\hline $5-4$ & CBM + Gyp & $\mathrm{S}$ & $0-6$ & $\mathrm{~A}$ & 7.1 & 2.61 & 12.1 & 13.4 & 3.63 & 4.2 & 5.8 & 0.73 & 22.4 \\
\hline $5-4$ & CBM + Gyp & $S$ & $6-11$ & Bt1 & 7.2 & 1.73 & 5.58 & 10.9 & 3.76 & 2.1 & 4.5 & 0.83 & 14.0 \\
\hline $5-4$ & CBM + Gyp & $\mathrm{S}$ & $11-24$ & Bt2 & 7.2 & 1.80 & 2.68 & 13.9 & 5.86 & 0.85 & 2.5 & 0.61 & 18.0 \\
\hline $6-1$ & CBM + Gyp & NT & $0-6.5$ & A & 7.0 & 1.99 & 17.5 & 5.21 & 1.66 & 9.5 & 14.6 & 0.96 & 5.06 \\
\hline $6-1$ & CBM + Gyp & NT & $6.5-11$ & Bt1 & 7.1 & 1.04 & 5.78 & 4.91 & 1.45 & 3.2 & 4.8 & 0.11 & 5.70 \\
\hline $6-1$ & CBM + Gyp & NT & $11-24$ & Bt2 & 7.1 & 1.09 & 2.22 & 7.44 & 2.39 & 1.0 & 2.7 & $-\mathrm{ND}$ & 8.36 \\
\hline $6-2$ & CBM + Gyp & $\mathrm{S}$ & $0-5.75$ & $\mathrm{~A}$ & 7.0 & 2.57 & 15.9 & 9.21 & 2.44 & 6.6 & 10.8 & 0.66 & 14.7 \\
\hline $6-2$ & CBM + Gyp & S & $5.75-11$ & Bt1 & 7.1 & 1.73 & 9.13 & 6.59 & 1.95 & 4.4 & 7.9 & 0.77 & 7.39 \\
\hline $6-2$ & CBM + Gyp & $\mathrm{S}$ & $11-24$ & $\mathrm{Bt} 2$ & 7.0 & 2.21 & 3.67 & 14.4 & 4.93 & 1.2 & 3.7 & 1.20 & 13.6 \\
\hline $6-3$ & CBM + Gyp & GS & $0-6$ & $\mathrm{~A}$ & 7.1 & 2.92 & 15.8 & 12.9 & 3.19 & 5.6 & 10.5 & 0.28 & 19.8 \\
\hline $6-3$ & CBM + Gyp & GS & $6-12$ & Bt1 & 7.1 & 1.91 & 5.91 & 11.1 & 3.88 & 2.2 & 4.4 & $-\mathrm{ND}$ & 14.4 \\
\hline $6-3$ & CBM + Gyp & GS & $12-24$ & Bt2 & 7.1 & 2.92 & 2.97 & 21.2 & 9.43 & 0.76 & 3.3 & $-\mathrm{ND}$ & 28.9 \\
\hline $6-4$ & CBM + Gyp & $\mathrm{G}$ & $0-6.25$ & $\mathrm{~A}$ & 6.8 & 2.35 & 14.6 & 9.21 & 2.12 & 6.2 & 14.4 & 0.22 & 5.93 \\
\hline $6-4$ & CBM + Gyp & G & $6.25-10$ & Bt1 & 7.1 & 1.85 & 7.03 & 10.6 & 3.38 & 2.7 & 6.8 & $-\mathrm{ND}$ & 12.1 \\
\hline $6-4$ & CBM + Gyp & G & $10-24$ & Bt2 & 7.0 & 2.81 & 2.66 & 22.7 & 9.50 & 0.66 & 3.8 & $-\mathrm{ND}$ & 29.2 \\
\hline 7-1 & CBM + Gyp + SB & $\mathrm{S}$ & $0-4.75$ & $\mathrm{~A}$ & 6.9 & 3.61 & 15.2 & 21.4 & 5.60 & 4.2 & 8.1 & 0.41 & 32.8 \\
\hline $7-1$ & $\mathrm{CBM}+\mathrm{Gyp}+\mathrm{SB}$ & $\mathrm{S}$ & $4.75-11$ & Bt1 & 7.1 & 2.34 & 9.74 & 12.6 & 3.70 & 3.4 & 4.7 & 0.32 & 18.6 \\
\hline $7-1$ & $\mathrm{CBM}+\mathrm{Gyp}+\mathrm{SB}$ & $\mathrm{S}$ & $11-24$ & $\mathrm{Bt} 2$ & 7.1 & 2.52 & 4.39 & 18.4 & 6.59 & 1.2 & 4.2 & 0.16 & 24.3 \\
\hline $7-2$ & CBM + Gyp + SB & NT & $0-5$ & A & 7.1 & 2.01 & 13.8 & 7.61 & 1.88 & 6.3 & 8.6 & 0.44 & 11.1 \\
\hline $7-2$ & $\mathrm{CBM}+\mathrm{Gyp}+\mathrm{SB}$ & NT & $5-12.5$ & Bt1 & 7.1 & 1.89 & 8.55 & 9.92 & 2.98 & 3.4 & 5.0 & 0.29 & 13.8 \\
\hline $7-2$ & $\mathrm{CBM}+\mathrm{Gyp}+\mathrm{SB}$ & NT & $12.5-24$ & $\mathrm{Bt} 2$ & 7.2 & 1.81 & 3.62 & 12.1 & 4.04 & 1.3 & 2.6 & $-\mathrm{ND}$ & 15.5 \\
\hline
\end{tabular}




\begin{tabular}{|c|c|c|c|c|c|c|c|c|c|c|c|c|c|}
\hline $7-3$ & $\mathrm{CBM}+\mathrm{Gyp}+\mathrm{SB}$ & GS & $0-5$ & A & 7.0 & 3.60 & 17.8 & 17.5 & 3.29 & 5.5 & 7.2 & 0.91 & 27.9 \\
\hline $7-3$ & $\mathrm{CBM}+\mathrm{Gyp}+\mathrm{SB}$ & GS & $5-12$ & Bt1 & 7.2 & 2.25 & 12.9 & 11.6 & 2.95 & 4.8 & 4.6 & 0.77 & 19.1 \\
\hline $7-3$ & CBM + Gyp + SB & GS & $12-24$ & Bt2 & 7.2 & 2.18 & 4.46 & 16.9 & 5.38 & 1.3 & 2.5 & 0.97 & 21.7 \\
\hline $7-4$ & $\mathrm{CBM}+\mathrm{Gyp}+\mathrm{SB}$ & $\mathrm{G}$ & $0-4.5$ & A & 7.0 & 4.41 & 21.2 & 28.4 & 5.11 & 5.2 & 7.2 & 1.61 & 41.9 \\
\hline $7-4$ & $\mathrm{CBM}+\mathrm{Gyp}+\mathrm{SB}$ & G & $4.5-12$ & Bt1 & 7.1 & 3.46 & 14.2 & 22.2 & 6.17 & 3.8 & 4.6 & 1.27 & 32.5 \\
\hline $7-4$ & CBM + Gyp + SB & G & $12-24$ & Bt2 & 7.2 & 3.03 & 6.19 & 22.9 & 8.18 & 1.6 & 2.5 & 0.85 & 31.6 \\
\hline 8-1 & CBM + Gyp + SB & G & $0-4$ & $\mathrm{~A}$ & 7.1 & 4.12 & 17.4 & 28.1 & 5.41 & 4.2 & 6.0 & 2.54 & 38.8 \\
\hline $8-1$ & $\mathrm{CBM}+\mathrm{Gyp}+\mathrm{SB}$ & G & $4-10.5$ & Bt1 & 7.1 & 3.02 & 11.4 & 19.5 & 5.18 & 3.2 & 4.0 & 1.31 & 27.3 \\
\hline $8-1$ & $\mathrm{CBM}+\mathrm{Gyp}+\mathrm{SB}$ & G & $10.5-24$ & Bt2 & 7.2 & 2.49 & 3.95 & 21.3 & 6.52 & 1.1 & 2.8 & 0.87 & 25.4 \\
\hline $8-2$ & CBM + Gyp + SB & $\mathrm{S}$ & $0-4.5$ & A & 7.1 & 2.99 & 17.5 & 12.8 & 2.98 & 6.2 & 8.4 & 1.41 & 20.9 \\
\hline $8-2$ & $\mathrm{CBM}+\mathrm{Gyp}+\mathrm{SB}$ & $S$ & $4.5-9.5$ & Bt1 & 7.3 & 2.24 & 13.9 & 9.46 & 2.41 & 5.7 & 4.5 & 1.66 & 16.7 \\
\hline $8-2$ & $\mathrm{CBM}+\mathrm{Gyp}+\mathrm{SB}$ & $\mathrm{S}$ & $9.5-24$ & Bt2 & 7.2 & 2.01 & 5.02 & 14.1 & 4.44 & 1.6 & 2.9 & 0.89 & 18.0 \\
\hline $8-3$ & $\mathrm{CBM}+\mathrm{Gyp}+\mathrm{SB}$ & GS & $0-4.5$ & A & 7.1 & 3.61 & 16.4 & 30.5 & 4.96 & 3.9 & 6.2 & 1.33 & 40.4 \\
\hline $8-3$ & $\mathrm{CBM}+\mathrm{Gyp}+\mathrm{SB}$ & GS & $4.5-11.5$ & Bt1 & 7.2 & 2.41 & 11.9 & 13.6 & 3.50 & 4.1 & 4.4 & 0.89 & 20.5 \\
\hline $8-3$ & $\mathrm{CBM}+\mathrm{Gyp}+\mathrm{SB}$ & GS & $11.5-24$ & Bt2 & 7.2 & 2.48 & 5.97 & 17.7 & 6.04 & 1.7 & 2.6 & 0.77 & 23.8 \\
\hline $8-4$ & $\mathrm{CBM}+\mathrm{Gyp}+\mathrm{SB}$ & NT & $0-4.25$ & $\mathrm{~A}$ & 7.0 & 1.92 & 14.1 & 8.82 & 2.12 & 6.0 & 10.2 & 1.11 & 11.8 \\
\hline $8-4$ & $\mathrm{CBM}+\mathrm{Gyp}+\mathrm{SB}$ & NT & $4.25-9.5$ & Bt1 & 7.2 & 1.63 & 8.52 & 9.06 & 2.48 & 3.6 & 5.0 & 0.76 & 12.3 \\
\hline $8-4$ & CBM + Gyp + SB & NT & $9.5-24$ & Bt2 & 7.2 & 1.78 & 4.01 & 13.5 & 4.42 & 1.3 & 3.2 & 0.71 & 16.4 \\
\hline $9-1$ & $\mathrm{CBM}+\mathrm{Gyp}+\mathrm{SB}$ & G & $0-4$ & $\mathrm{~A}$ & 6.7 & 3.71 & 11.4 & 30.7 & 5.24 & 2.7 & 7.5 & 0.93 & 37.6 \\
\hline $9-1$ & $\mathrm{CBM}+\mathrm{Gyp}+\mathrm{SB}$ & G & $4-9.5$ & Bt1 & 6.9 & 3.69 & 10.3 & 30.7 & 9.30 & 2.3 & 5.5 & 0.75 & 41.6 \\
\hline $9-1$ & CBM + Gyp + SB & $\mathrm{G}$ & $9.5-24$ & Bt2 & 7.1 & 2.14 & 3.32 & 19.1 & 6.74 & 0.92 & 2.0 & 0.51 & 25.2 \\
\hline $9-2$ & CBM + Gyp + SB & GS & $0-4.5$ & A & 6.8 & 3.77 & 13.1 & 30.7 & 6.31 & 3.0 & 7.5 & 0.94 & 41.9 \\
\hline $9-2$ & $\mathrm{CBM}+\mathrm{Gyp}+\mathrm{SB}$ & GS & $4.5-9$ & Bt1 & 7.0 & 2.89 & 9.61 & 21.3 & 5.79 & 2.6 & 4.0 & 0.74 & 30.4 \\
\hline $9-2$ & $\mathrm{CBM}+\mathrm{Gyp}+\mathrm{SB}$ & GS & $9-24$ & Bt2 & 7.1 & 2.62 & 3.65 & 23.3 & 7.44 & 0.93 & 2.2 & 0.75 & 29.5 \\
\hline $9-3$ & CBM + Gyp + SB & $\mathrm{S}$ & $0-4$ & $\mathrm{~A}$ & 7.0 & 3.19 & 11.6 & 21.1 & 5.36 & 3.2 & 7.1 & 0.74 & 30.6 \\
\hline $9-3$ & CBM + Gyp + SB & S & $4-9.5$ & Bt1 & 7.1 & 1.72 & 7.86 & 9.69 & 2.72 & 3.2 & 4.5 & 0.59 & 14.5 \\
\hline $9-3$ & $\mathrm{CBM}+\mathrm{Gyp}+\mathrm{SB}$ & $\mathrm{S}$ & $9.5-24$ & $\mathrm{Bt} 2$ & 7.2 & 1.59 & 3.32 & 13.1 & 4.53 & 1.1 & 2.5 & 0.48 & 16.6 \\
\hline
\end{tabular}




\begin{tabular}{|c|c|c|c|c|c|c|c|c|c|c|c|c|c|}
\hline $9-4$ & $\mathrm{CBM}+\mathrm{Gyp}+\mathrm{SB}$ & NT & $0-5$ & A & 7.0 & 2.30 & 12.5 & 12.7 & 3.15 & 4.4 & 7.2 & 1.00 & 18.8 \\
\hline $9-4$ & $\mathrm{CBM}+\mathrm{Gyp}+\mathrm{SB}$ & NT & $5-11$ & Bt1 & 7.1 & 1.83 & 5.86 & 13.6 & 4.12 & 2.0 & 3.0 & 0.60 & 19.1 \\
\hline $9-4$ & $\mathrm{CBM}+\mathrm{Gyp}+\mathrm{SB}$ & NT & $11-24$ & Bt2 & 7.2 & 1.68 & 2.78 & 13.3 & 4.63 & 0.93 & 2.0 & 0.57 & 17.0 \\
\hline $10-1$ & $\mathrm{CBM}+\mathrm{Gyp}+\mathrm{SB}$ & $\mathrm{S}$ & $0-5.5$ & A & 6.9 & 2.70 & 12.2 & 14.7 & 4.11 & 4.0 & 7.8 & 0.67 & 23.9 \\
\hline $10-1$ & $\mathrm{CBM}+\mathrm{Gyp}+\mathrm{SB}$ & $\mathrm{S}$ & $5.5-11.5$ & Bt1 & 8.44 & 2.14 & 7.09 & 13.39 & 4.35 & 2.38 & 5.4 & 0.46 & 18.44 \\
\hline $10-1$ & $\mathrm{CBM}+\mathrm{Gyp}+\mathrm{SB}$ & $\mathrm{S}$ & $11.5-24$ & Bt2 & 8.44 & 2.14 & 2.79 & 17.81 & 6.89 & 0.80 & 2.6 & 0.43 & 22.25 \\
\hline $10-2$ & $\mathrm{CBM}+\mathrm{Gyp}+\mathrm{SB}$ & G & $0-6$ & A & 6.7 & 3.35 & 10.1 & 26.6 & 4.99 & 2.5 & 6.0 & 0.67 & 35.4 \\
\hline $10-2$ & $\mathrm{CBM}+\mathrm{Gyp}+\mathrm{SB}$ & G & $6-11.5$ & Bt1 & 6.8 & 2.94 & 5.36 & 22.3 & 6.69 & 1.4 & 3.7 & 0.63 & 29.9 \\
\hline $10-2$ & $\mathrm{CBM}+\mathrm{Gyp}+\mathrm{SB}$ & G & $11.5-24$ & Bt2 & 6.9 & 2.63 & 2.96 & 22.2 & 8.97 & 0.75 & 2.8 & 0.71 & 30.9 \\
\hline $10-3$ & $\mathrm{CBM}+\mathrm{Gyp}+\mathrm{SB}$ & NT & $0-6.25$ & A & 7.0 & 1.74 & 10.6 & 6.28 & 1.61 & 5.3 & 7.6 & 0.97 & 9.57 \\
\hline $10-3$ & $\mathrm{CBM}+\mathrm{Gyp}+\mathrm{SB}$ & NT & $6.25-12.5$ & Bt1 & 6.7 & 1.67 & 4.97 & 11.0 & 3.48 & 1.8 & 8.2 & 0.68 & 9.14 \\
\hline $10-3$ & $\mathrm{CBM}+\mathrm{Gyp}+\mathrm{SB}$ & NT & $12.5-24$ & Bt2 & 7.0 & 1.74 & 2.13 & 13.6 & 5.04 & 0.70 & 2.6 & 0.44 & 16.8 \\
\hline $10-4$ & $\mathrm{CBM}+\mathrm{Gyp}+\mathrm{SB}$ & GS & $0-6$ & A & 6.8 & 3.89 & 12.5 & 25.5 & 5.32 & 3.2 & 7.4 & 1.08 & 39.2 \\
\hline $10-4$ & $\mathrm{CBM}+\mathrm{Gyp}+\mathrm{SB}$ & GS & $6-12$ & Bt1 & 6.9 & 2.96 & 8.06 & 21.0 & 6.01 & 2.2 & 3.8 & 0.71 & 31.2 \\
\hline $10-4$ & $\mathrm{CBM}+\mathrm{Gyp}+\mathrm{SB}$ & GS & $12-24$ & Bt2 & 6.9 & 2.88 & 2.85 & 24.0 & 9.10 & 0.70 & 2.9 & 0.65 & 31.6 \\
\hline $11-1$ & PC & NT & $0-6$ & A & 8.73 & 1.036 & 1.43 & 8.11 & 2.66 & 0.62 & 6.6 & 0.43 & 1.74 \\
\hline $11-1$ & PC & NT & $6-11$ & Bt1 & 8.61 & 0.781 & 1.18 & 5.93 & 1.99 & 0.59 & 4.8 & 0.19 & 2.26 \\
\hline $11-1$ & PC & NT & $11-24$ & Bt2 & 8.34 & 0.8 & 1.32 & 5.59 & 1.98 & 0.68 & 3.8 & 0.21 & 4.09 \\
\hline $11-2$ & PC & GS & $0-6$ & A & 6.9 & 1.84 & 1.62 & 15.9 & 4.42 & 0.51 & 5.5 & 0.53 & 18.6 \\
\hline $11-2$ & PC & GS & $6-11$ & Bt1 & 6.9 & 1.63 & 1.62 & 13.0 & 4.23 & 0.55 & 3.2 & 0.47 & 14.7 \\
\hline $11-2$ & PC & GS & $11-24$ & Bt2 & 7.0 & 2.36 & 2.22 & 18.4 & 6.49 & 0.63 & 2.4 & 0.37 & 24.3 \\
\hline $11-3$ & PC & $\mathrm{S}$ & $0-5.5$ & A & 8.57 & 1.316 & 1.17 & 10.56 & 3.30 & 0.44 & 5.4 & 0.32 & 7.59 \\
\hline $11-3$ & PC & $\mathrm{S}$ & $5.5-8.5$ & Bt1 & 8.44 & 1.067 & 1.12 & 8.06 & 2.91 & 0.48 & 5 & 0.32 & 5.62 \\
\hline $11-3$ & PC & S & $8.5-24$ & Bt2 & 7.0 & 1.01 & 1.33 & 7.76 & 2.85 & 0.58 & 3.8 & 0.30 & 7.25 \\
\hline $11-4$ & PC & G & $0-6$ & A & 6.7 & 1.71 & 1.55 & 16.4 & 3.84 & 0.49 & 7.4 & 0.38 & 13.4 \\
\hline $11-4$ & PC & G & $6-11.5$ & Bt1 & 6.7 & 1.76 & 1.52 & 15.6 & 4.95 & 0.47 & 4.7 & 0.43 & 16.8 \\
\hline $11-4$ & PC & G & $11.5-24$ & Bt2 & 6.9 & 2.76 & 2.31 & 24.1 & 8.69 & 0.57 & 3.4 & 0.28 & 31.7 \\
\hline
\end{tabular}




\begin{tabular}{|c|c|c|c|c|c|c|c|c|c|c|c|c|c|}
\hline $12-1$ & PC & NT & $0-5.5$ & A & 6.7 & 0.86 & 1.49 & 7.01 & 2.17 & 0.70 & 8.3 & 0.45 & 1.49 \\
\hline $12-1$ & PC & NT & $5.5-12$ & Bt1 & 6.9 & 0.74 & 1.02 & 3.96 & 1.42 & 0.62 & 4.0 & 1.10 & 1.48 \\
\hline $12-1$ & PC & NT & $12-24$ & Bt2 & 7.1 & 0.74 & 1.9 & 5.7 & 1.9 & 0.99 & 3.1 & 0.03 & 4.8 \\
\hline $12-2$ & PC & $\mathrm{S}$ & $0-6.5$ & A & 7.0 & 1.65 & 1.50 & 11.5 & 3.90 & 0.54 & 5.1 & 0.38 & 11.6 \\
\hline $12-2$ & PC & $\mathrm{S}$ & $6.5-11$ & Bt1 & 6.9 & 1.02 & 1.39 & 6.88 & 2.42 & 0.64 & 3.8 & 0.27 & 6.66 \\
\hline $12-2$ & PC & $\mathrm{S}$ & $11-24$ & Bt2 & 7.0 & 1.35 & 2.14 & 10.7 & 3.91 & 0.79 & 3.2 & 0.32 & 12.7 \\
\hline $12-3$ & PC & G & $0-5$ & A & 6.8 & 1.72 & 1.46 & 16.6 & 4.03 & 0.45 & 6.1 & 0.42 & 15.9 \\
\hline $12-3$ & PC & G & $5-11.5$ & Bt1 & 6.9 & 1.91 & 1.73 & 18.3 & 6.28 & 0.49 & 3.8 & 0.29 & 21.8 \\
\hline $12-3$ & PC & G & $1.5-24$ & Bt2 & 7.0 & 1.96 & 2.07 & 17.0 & 7.13 & 0.60 & 2.6 & 0.27 & 23.0 \\
\hline $12-4$ & PC & GS & $0-6$ & A & 6.9 & 2.44 & 1.60 & 23.4 & 5.08 & 0.42 & 5.1 & 0.39 & 26.2 \\
\hline $12-4$ & PC & GS & $6-11$ & Bt1 & 6.9 & 1.56 & 1.72 & 13.5 & 3.93 & 0.58 & 3.9 & 0.41 & 14.1 \\
\hline $12-4$ & PC & GS & $11-24$ & Bt2 & 7.0 & 1.97 & 1.92 & 17.6 & 6.21 & 0.56 & 2.3 & 0.34 & 22.5 \\
\hline 13-1 & CBM & GS & $0-5$ & A & 6.9 & 3.10 & 16.3 & 13.0 & 3.22 & 5.7 & 8.4 & 2.34 & 23.1 \\
\hline 13-1 & CBM & GS & $5-12$ & Bt1 & 7.1 & 1.64 & 8.43 & 7.93 & 2.44 & 3.7 & 6.4 & 1.10 & 10.8 \\
\hline $13-1$ & CBM & GS & $12-24$ & Bt2 & 7.1 & 1.55 & 3.55 & 10.9 & 4.30 & 1.3 & 2.8 & 0.63 & 14.9 \\
\hline $13-2$ & CBM & NT & $0-5.5$ & A & 6.9 & 1.79 & 15.0 & 5.37 & 1.85 & 7.9 & 14.7 & 1.36 & 1.79 \\
\hline $13-2$ & CBM & NT & $5.5-12$ & Bt1 & 7.0 & 0.96 & 3.08 & 6.80 & 2.35 & 1.4 & 5.7 & 2.43 & 2.59 \\
\hline $13-2$ & CBM & NT & $12-24$ & Bt2 & 7.2 & 0.94 & 2.62 & 7.45 & 2.77 & 1.2 & 4.6 & 0.64 & 6.91 \\
\hline $13-3$ & CBM & S & $0-5$ & A & 7.1 & 3.53 & 19.0 & 13.7 & 4.37 & 6.3 & 6.9 & 1.04 & 30.7 \\
\hline $13-3$ & CBM & S & $5-10.5$ & Bt1 & 7.1 & 1.48 & 7.73 & 6.91 & 2.38 & 3.6 & 6.0 & 1.68 & 8.55 \\
\hline $13-3$ & CBM & S & $10.5-24$ & Bt2 & 7.3 & 1.01 & 2.59 & 7.43 & 2.90 & 1.1 & 2.2 & 0.58 & 8.98 \\
\hline $13-4$ & CBM & G & $0-6$ & A & 7.1 & 2.98 & 12.1 & 10.1 & 2.53 & 4.8 & 10.4 & 1.05 & 13.8 \\
\hline $13-4$ & CBM & G & $6-12$ & Bt1 & 6.9 & 1.81 & 6.09 & 11.3 & 4.09 & 2.2 & 5.1 & 1.77 & 13.5 \\
\hline $13-4$ & CBM & G & $12-24$ & Bt2 & 7.1 & 2.26 & 3.36 & 15.7 & 7.19 & 0.99 & 2.9 & 0.53 & 22.6 \\
\hline $14-1$ & CBM & GS & $0-5.75$ & A & 7.0 & 2.6 & 12.5 & 12.9 & 3.5 & 4.4 & 5.3 & 1.2 & 17.5 \\
\hline $14-1$ & CBM & GS & $5.75-11$ & Bt1 & 7.1 & 1.7 & 5.2 & 10.4 & 3.6 & 2.0 & 4.9 & 0.7 & 11.8 \\
\hline $14-1$ & CBM & GS & $11-24$ & Bt2 & 8.45 & 2.2 & 2.44 & 17.99 & 7.90 & 0.68 & 3.2 & 0.33 & 20.56 \\
\hline
\end{tabular}




\begin{tabular}{|c|c|c|c|c|c|c|c|c|c|c|c|c|c|}
\hline $14-2$ & CBM & $\mathrm{S}$ & $0-5.5$ & A & 7.0 & 1.9 & 10.9 & 7.9 & 2.9 & 4.7 & 6.4 & 0.9 & 11.6 \\
\hline $14-2$ & CBM & $\mathrm{S}$ & 5.512 & Bt1 & 7.0 & 1.1 & 4.0 & 5.6 & 2.5 & 2.0 & 5.5 & 0.5 & 4.9 \\
\hline $14-2$ & CBM & $\mathrm{S}$ & $12-24$ & Bt2 & 7.2 & 0.95 & 2.6 & 5.6 & 2.7 & 1.3 & 4.4 & 0.5 & 5.0 \\
\hline $14-3$ & CBM & G & $0-5$ & A & 7.1 & 1.7 & 14.2 & 4.5 & 1.3 & 8.3 & 10.2 & 1.1 & 4.3 \\
\hline $14-3$ & CBM & G & $5-11$ & Bt1 & 7.1 & 1.0 & 5.5 & 4.9 & 2.0 & 3.0 & 5.6 & 0.7 & 4.7 \\
\hline $14-3$ & CBM & G & $11-24$ & Bt2 & 7.2 & 1.6 & 2.5 & 9.7 & 4.7 & 0.92 & 2.3 & 0.4 & 13.3 \\
\hline $14-4$ & CBM & NT & $0-5.75$ & A & 7.3 & 1.4 & 11.6 & 2.8 & 0.9 & 8.6 & 9.2 & 1.6 & 1.7 \\
\hline $14-4$ & CBM & NT & $5.75-11$ & Bt1 & 7.3 & 0.69 & 3.2 & 3.3 & 1.2 & 2.1 & 4.8 & 0.8 & 1.2 \\
\hline $14-4$ & CBM & NT & $11-24$ & Bt2 & 7.3 & 0.54 & 2.1 & 3.0 & 1.3 & 1.4 & 3.6 & 0.5 & 1.8 \\
\hline $15-1$ & CBM + Gyp & G & $0-6$ & A & 7.0 & 2.5 & 16.3 & 10.3 & 2.1 & 6.6 & 6.9 & 1.2 & 17.1 \\
\hline $15-1$ & CBM + Gyp & G & 6-12 & Bt1 & 7.2 & 1.5 & 8.8 & 6.6 & 2.3 & 4.2 & 5.0 & 0.8 & 10 \\
\hline $15-1$ & CBM + Gyp & G & $12-24$ & Bt2 & 7.1 & 2.2 & 4.2 & 14.2 & 6.0 & 1.3 & 2.1 & 0.6 & 19.8 \\
\hline $15-2$ & CBM + Gyp & NT & $0-6$ & A & 7.1 & 1.7 & 14.0 & 4.7 & 1.7 & 7.8 & 9.8 & 1.2 & 2.4 \\
\hline $15-2$ & CBM + Gyp & NT & $6-12$ & Bt1 & 7.1 & 0.97 & 6.7 & 3.9 & 1.5 & 4.1 & 6.2 & 0.8 & 3.3 \\
\hline $15-2$ & CBM + Gyp & NT & $12-24$ & Bt2 & 7.1 & 0.94 & 2.8 & 6.2 & 2.9 & 1.3 & 4.4 & 0.6 & 5.8 \\
\hline $15-3$ & CBM + Gyp & $\mathrm{S}$ & $0-6$ & A & 8.77 & 2.18 & 14.29 & 8.68 & 2.78 & 5.97 & 7 & 0.66 & 13.85 \\
\hline $15-3$ & CBM + Gyp & S & $6-12$ & Bt1 & 8.53 & 1.549 & 6.16 & 7.84 & 3.09 & 2.63 & 3.6 & 0.74 & 11.84 \\
\hline $15-3$ & CBM + Gyp & $\mathrm{S}$ & $12-24$ & Bt2 & 8.43 & 1.621 & 2.24 & 12.29 & 5.21 & 0.76 & 3.2 & 0.45 & 15.57 \\
\hline $15-4$ & CBM + Gyp & GS & $0-5$ & A & 8.72 & 2.14 & 12.15 & 11.14 & 3.00 & 4.57 & 7.4 & 1.00 & 12.11 \\
\hline $15-4$ & CBM + Gyp & GS & $5-11$ & Bt1 & 8.66 & 1.469 & 6.96 & 7.45 & 2.79 & 3.07 & 4 & 0.54 & 9.17 \\
\hline $15-4$ & CBM + Gyp & GS & $11-24$ & Bt2 & 8.47 & 1.55 & 4.15 & 9.88 & 4.23 & 1.56 & 3.4 & 0.49 & 13.53 \\
\hline $16-1$ & CBM + Gyp & G & $0-5$ & A & 8.87 & 1.669 & 12.19 & 6.11 & 1.98 & 6.06 & 4.6 & 0.87 & 2.68 \\
\hline $16-1$ & CBM + Gyp & G & $5-12$ & Bt1 & 8.55 & 1.213 & 5.31 & 5.94 & 2.47 & 2.59 & 5.6 & 0.52 & 6.05 \\
\hline $16-1$ & CBM + Gyp & G & $12-24$ & Bt2 & 8.44 & 1.466 & 2.18 & 10.18 & 4.91 & 0.79 & 3 & 0.35 & 13.09 \\
\hline $16-2$ & CBM + Gyp & S & $0-5$ & A & 8.72 & 2.18 & 14.57 & 8.84 & 3.21 & 5.93 & 10.4 & 0.91 & 8.75 \\
\hline $16-2$ & CBM + Gyp & S & $10-5$ & Bt1 & 8.76 & 1.192 & 3.86 & 6.72 & 2.84 & 1.77 & 4.2 & 0.45 & 7.82 \\
\hline $16-2$ & CBM + Gyp & S & $10.5-24$ & Bt2 & 8.43 & 1.366 & 1.82 & 9.80 & 4.85 & 0.67 & 3.2 & 0.45 & 11.79 \\
\hline
\end{tabular}




\begin{tabular}{|c|c|c|c|c|c|c|c|c|c|c|c|c|c|}
\hline $16-3$ & CBM + Gyp & NT & $0-6$ & A & 7.0 & 1.7 & 13.7 & 4.4 & 1.5 & 8.0 & 12.2 & 1.2 & 2.2 \\
\hline $16-3$ & CBM + Gyp & NT & $6-11.5$ & Bt1 & 8.38 & 1.944 & 8.33 & 11.29 & 4.60 & 2.96 & 3.4 & 2.45 & 5.59 \\
\hline $16-3$ & CBM + Gyp & NT & $11.5-24$ & Bt2 & 7.1 & 1.1 & 2.7 & 7.8 & 3.5 & 1.1 & 4.1 & 0.6 & 8.0 \\
\hline $16-4$ & CBM + Gyp & GS & $0-5$ & A & 7.1 & 2.5 & 13.2 & 12.4 & 3.4 & 4.7 & 7.1 & 1.2 & 17.6 \\
\hline $16-4$ & CBM + Gyp & GS & $5-12$ & Bt1 & 8.43 & 2.63 & 7.47 & 18.47 & 6.88 & 2.10 & 3.4 & 0.57 & 24.06 \\
\hline $16-4$ & CBM + Gyp & GS & $12-24$ & Bt2 & 8.38 & 2.5 & 3.07 & 20.85 & 9.39 & 0.79 & 2.8 & 0.37 & 26.40 \\
\hline $17-1$ & CBM PC Blend & GS & $0-6$ & A & 6.8 & 2.0 & 9.6 & 8.8 & 3.2 & 3.9 & 7.7 & 3.3 & 12.2 \\
\hline $17-1$ & CBM PC Blend & GS & $6-16$ & Bt1 & 8.62 & 1.188 & 3.78 & 6.80 & 3.12 & 1.70 & 5.8 & 0.64 & 5.35 \\
\hline $17-1$ & CBM PC Blend & GS & $16-24$ & Bt2 & 8.52 & 1.634 & 3.11 & 10.31 & 5.98 & 1.09 & 4.2 & 0.39 & 13.04 \\
\hline $17-2$ & CBM PC Blend & S & $0-6$ & A & 8.79 & 1.466 & 7.71 & 6.73 & 2.81 & 3.53 & 7.8 & 0.83 & 4.81 \\
\hline $17-2$ & CBM PC Blend & $\mathrm{S}$ & $6-16$ & Bt1 & 6.9 & 0.95 & 4.8 & 5.2 & 2.1 & 2.5 & 8.9 & 0.7 & 3.5 \\
\hline $17-2$ & CBM PC Blend & $\mathrm{S}$ & $16-24$ & Bt2 & 7.1 & 0.91 & 4.1 & 4.8 & 3.1 & 2.1 & 5.0 & 0.6 & 4.9 \\
\hline $17-3$ & CBM PC Blend & G & $0-6$ & A & 8.77 & 1.685 & 8.13 & 8.69 & 2.84 & 3.39 & 7.6 & 0.86 & 8.99 \\
\hline $17-3$ & CBM PC Blend & G & $6-14.5$ & Bt1 & 7.68 & 1.107 & 3.75 & 5.92 & 2.77 & 1.80 & 3.4 & 0.41 & 7.62 \\
\hline $17-3$ & CBM PC Blend & G & $14.5-24$ & Bt2 & 8.38 & 2.18 & 5.32 & 13.48 & 8.51 & 1.60 & 2.4 & 0.36 & 18.25 \\
\hline $17-4$ & CBM PC Blend & NT & $0-6$ & A & 8.82 & 1.501 & 6.72 & 4.64 & 2.19 & 3.64 & 8.2 & 1.25 & 2.60 \\
\hline $17-4$ & CBM PC Blend & NT & $6-20$ & Bt1 & 8.54 & 1.552 & 3.21 & 13.05 & 6.97 & 1.01 & 2.2 & 2.77 & 13.38 \\
\hline $17-4$ & CBM PC Blend & NT & $20-24$ & Bt2 & 8.66 & 1.22 & 3.92 & 5.87 & 3.72 & 1.79 & 3.4 & 1.34 & 7.24 \\
\hline $18-1$ & CBM PC Blend & GS & $0-6$ & A & 6.9 & 1.8 & 11.5 & 7.8 & 2.9 & 5.0 & 8.9 & 1.0 & 11.0 \\
\hline $18-1$ & CBM PC Blend & GS & $6-15$ & Bt1 & 8.71 & 0.8 & 2.40 & 4.27 & 1.87 & 1.37 & 2.6 & 0.39 & 4.71 \\
\hline $18-1$ & CBM PC Blend & GS & $15-24$ & Bt2 & 8.46 & 1.467 & 2.00 & 10.48 & 5.14 & 0.72 & 3 & 0.32 & 13.75 \\
\hline $18-2$ & CBM PC Blend & S & $0-6$ & A & 6.7 & 1.8 & 10 & 8.4 & 3.9 & 4.0 & 9.7 & 0.9 & 9.7 \\
\hline $18-2$ & CBM PC Blend & $S$ & $6-17$ & Bt1 & 6.9 & 0.90 & 3.2 & 6.0 & 2.7 & 1.6 & 5.2 & 0.7 & 4.6 \\
\hline $18-2$ & CBM PC Blend & $\mathrm{S}$ & $17-24$ & Bt2 & 6.9 & 1.3 & 3.6 & 7.5 & 4.1 & 1.5 & 4.4 & 0.6 & 8.8 \\
\hline $18-3$ & CBM PC Blend & G & $0-5$ & A & 6.8 & 1.4 & 7.4 & 5.8 & 2.1 & 3.8 & 9.0 & 1.0 & 7.0 \\
\hline $18-3$ & CBM PC Blend & G & $5-24$ & Bt1 & 6.9 & 1.4 & 5.1 & 7.1 & 4.9 & 2.1 & 2.4 & 0.9 & 13.5 \\
\hline $18-4$ & CBM PC Blend & NT & $0-6$ & A & 8.83 & 1.26 & 7.95 & 5.11 & 2.36 & 4.11 & 8.4 & 0.96 & 0.79 \\
\hline
\end{tabular}




\begin{tabular}{|c|c|c|c|c|c|c|c|c|c|c|c|}
\hline CBM PC Blend & NT & $6-14.5$ & Bt1 & 8.93 & 1.037 & 4.91 & 4.81 & 2.70 & 2.53 & 6 & 0.69 \\
\hline CBM PC Blend & NT & $14.5-24$ & Bt2 & 8.65 & 0.868 & 2.60 & 4.92 & 2.61 & 1.34 & 3.6 & 0.26 \\
\hline
\end{tabular}

